Doctoral Thesis for Ph. D. in Aerospace Engineering

\title{
Hydrodynamic processes in magnetized ablation fronts
}

\author{
by \\ Fernando García Rubio
}

Aerospace Engineer

Supervisor: Professor Francisco Javier Sanz Recio

September 2018

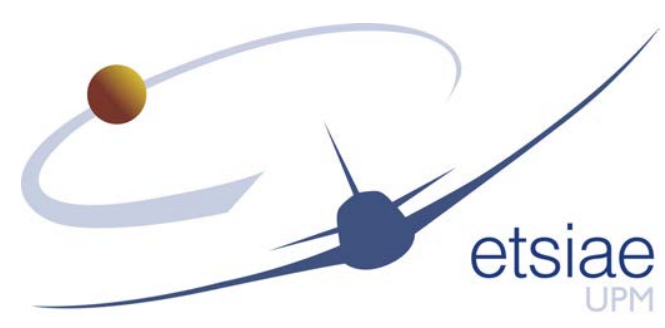

Departamento de Física Aplicada a las Ingenierías Naval y Aeronáutica

Escuela Técnica Superior de Ingeniería Aeronáutica y del Espacio 
Dean

Referees

Date of the graduation

26 October 2018 
Dedicated to my family 



\section{Acknowledgments}

First and foremost, I would like to express my deep gratitude to my advisor, Professor Francisco Javier Sanz Recio, for sharing his knowledge, time and experience during these years. His passion for science, savoir-faire, rigorous spirit and humbleness are valuable life lessons that I will never forget.

I would like to thank all the professors in the research group "Grupo de Investigación en Fusión Inercial y Física de Plasmas" for their continuous support and patience. I have a special thought for the secretary of the group, Mari Carmen, who has thoroughly helped me in the tedious bureaucratic tasks, and whom I wish all my best during these hard days. I would like to express my gratitude to all the $\mathrm{Ph}$. $\mathrm{D}$. students at the ETSIAE that shared with me countless hours in cafetería. I would like to mention the still young but soon to become a great researcher Jaime Paradela Díaz, whom I wish to encounter in a near future. I cannot forget to thank Osquitar and Silvia for their little cheer up every morning.

I would like to express my deep appreciation to Professor Riccardo Betti and Professor Masakatsu Murakami, who made possible and supervised my research stays at LLE in Rochester and ILE in Osaka, respectively, as well as all the people that helped me adapting to the American and Japanese cultures. I would also like to thank Professor Kunioki Mima for his support and fruitful discussions. Lastly, I sincerely thank and feel in debt with my family, especially my parents and my brother, for their continuous and unconditional support during all these years.

This thesis was supported by the Spanish Ministerio de Educación, Cultura y Deporte under the national research program FPU, grant FPU 14/04879, and by the Spanish Ministerio de Economía y Competitividad, Project No. ENE2014-54960R. 



\section{Abstract}

Magneto inertial fusion (MIF) schemes arise as a combination of the compression heating, characteristic of inertial confinement fusion, with the magnetic insulation and alpha heating enhancement typical of magnetic confinement fusion. Among them, the recently proposed magnetized liner inertial fusion (MagLIF) scheme has gained great attention in the last decade. It consists of magnetizing a cylindrical target and preheating the fuel prior to compression. Both pulsed-power and laser driven implosions have been experimentally tested, and significant increment in the neutron yield when the target was magnetized has been measured. This places MagLIF as an attractive alternative scheme in fusion research.

In this thesis, several hydrodynamic processes appearing in magneto inertial fusion have been investigated. The approach taken is based on the proposition of simple problems allowing for analytical resolution which capture the phenomenon subject of study. Apart from the physical insight obtained, the analytical solutions serve as valuable test cases to benchmark magnetohydrodynamics codes.

The hydrodynamic processes studied in this thesis concern the evolution of the corona expansion of magnetized targets, the cooling wave developing at the interface between the magnetized hot spot and the cold unmagnetized liner enclosing it, the effects of the magnetic pressure in MagLIF fusion-like plasmas, fuel pollution due to mass diffusion, magnetic flux conservation in an imploding plasma in the subsonic regime and, finally, alpha heating enhancement due to magnetization and its effect on the ignition physics.

The bulk of the thesis is devoted to the study of the magnetic flux conservation in an imploding plasma, which in turn affects the implosion hydrodynamics. The magnetic field is convected by two opposite mechanisms: plasma motion inside the hot spot, which convects it towards the center, and the thermoelectric Nernst term, which convects the magnetic field towards the liner. The overall convection direction depends on the magnetization level of the hot spot. When it is small, the Nernst term dominates and the magnetic field is convected out of the fuel, it accumulates close to the liner and is diffused in a thin layer. The Nernst term acts as an engine that degrades the magnetic flux conservation independently of how conductive the hot spot is. However, magnetization of the hot spot suppresses the Nernst term and improves the magnetic flux conservation. The center of the hot spot becomes magnetically insulated and undergoes an ideal adiabatic implosion. This desirable state is only attained if the initial parameters satisfy a certain requirement, expressed 
in terms of the initial electron Hall parameter and the initial Péclet and magnetic Lewis numbers.

Additionally, it is found that once the magnetic pressure becomes important, increasing further the magnetization level does not improve the magnetic flux conservation as effectively. Furthermore, the analysis of the fuel - liner mixing reveals that mass diffusion is confined within a thin layer compared to the characteristic width of the aforementioned cooling wave. Nevertheless, it may lead to a significant fuel pollution, even comparable to the one induced by microscopic mixing arisen from hydrodynamic instabilities. Finally, a dynamic ignition criterion for magnetized implosions is derived, in which the magnetic field effects on the alpha particles energy deposition is taken into account. It shows the importance of attaining a magnetization level high enough to magnetize the alpha particles. 


\section{Resumen}

Los esquemas de fusión llamados "magneto inertial fusion" (MIF) surgen como combinación del calentamiento por compresión, característico de la fusión por confinamiento inercial, con el aislamiento magnético e incremento de la deposición de energía de las partículas alpha, típico de la fusión por confinamiento magnético. De entre ellos, se ha prestado gran atención en la última década al recientemente propuesto "magnetized liner inertial fusion" (MagLIF). Este esquema consiste en magnetizar un blanco cilíndrico y precalentar el combustible antes de la compresión. Experimentalmente, se han realizado tanto implosiones comprimidas por máquinas de potencia pulsada como por láser, en las que se ha medido un incremento significativo en la producción de neutrones cuando el blanco se magnetiza. Este hecho muestra el atractivo de MagLIF como esquema alternativo para la investigación en fusión.

En esta tesis, se han investigado varios procesos hidrodinámicos que tienen lugar en esquemas de fusión MIF. El enfoque adoptado corresponde a la proposición de problemas simples, los cuales permiten una resolución analítica, que capturen el fenómeno sujeto a estudio. Además de la comprensión física que otorga su resolución, las soluciones analíticas son de gran interés para el testeo de códigos magnetohidrodinámicos.

Los procesos hidrodinámicos estudiados en esta tesis conciernen la evolución de la expansión de la corona en blancos magnetizado, la onda térmica que se desarrolla en la interfaz entre el núcleo caliente magnetizado y el revestimiento que lo envuelve, los efectos de presión magnética en un contexto MagLIF, la contaminación del combustible debido a la difusión másica, la conservación del flujo magnético en una implosión de plasma en régimen subsónico y, finalmente, el aumento del calentamiento por deposición de energía de las alphas debido al campo magnético y su efecto en la ignición.

El grueso de esta tesis se centra en el estudio de la conservación de campo magnético en una implosión de plasma, el cual afecta a su vez a la hidrodinámica de la misma. El campo magnético es conveccionado por dos mecanismos opuestos: el movimiento del plasma dentro del núcleo, el cual lo convecciona hacia el centro, y el efecto termoeléctrico Nernst, que lo convecciona hacia el revestimiento. El sentido neto de convección depende del nivel de magnetización del núcleo. Cuando éste es bajo, el efecto Nernst domina y el campo magnético es adveccionado fuera del combustible, se acumula cerca del revestimiento y es difundido en una capa delgada. El efecto Nernst promueve de este modo la pérdida del flujo de campo magnético independientemente 
de lo conductor que sea el núcleo. Sin embargo, cuando el núcleo está magnetizado, el término Nernst se reduce y se mejora la conservación del flujo. El centro del núcleo se aísla magnéticamente y experimenta una implosión adiabática ideal. A este estado de magnetización tan deseable sólo se puede llegar si los parámetros iniciales satisfacen cierto requerimeiento, el cual se expresa en términos del parámetro de Hall de los electrones y de los números de Péclet y del Lewis magnetizado iniciales.

Adicionalmente, se ha descubierto que una vez que la presión magnética es importante, incrementar más el nivel de magnetización no mejora la conservación de flujo magnético tan eficazmente. Por otra parte, el análisis del mezclado de combustible con material del revestimiento revela que la difusión másica está confinada en una capa delgada en comparación con el espesor característico de la onda térmica mencionada previamente. A pesar de ello, puede llevar a una contaminación del combustible significativa, incluso comparable a la que se induciría por un mezclado macroscópico surgido de inestabilidades hidrodinámicas. Finalmente, se ha desarrollado un criterio de ignición dinámico para implosiones magnetizadas, en el cual el efecto del campo magnético en la deposición de las partículas alpha se ha tenido en cuenta. Éste muestra la importancia de llegar a un nivel de magnetización en el estancamiento de la cápsula lo suficientemente alto como para magnetizar las partículas alpha. 


\section{Contents}

1 Introduction $\quad 1$

1.1 Thermonuclear fusion . . . . . . . . . . . . . . . . . . . 1

1.2 Inertial confinement fusion . . . . . . . . . . . . . . . . 2

1.2.1 Requirements for ignition . . . . . . . . . . . 3

1.2.2 Implosion asymmetries . . . . . . . . . . . . . . . . . . . 4

1.3 Magneto inertial fusion . . . . . . . . . . . . . . . . . 4

1.3.1 Magnetic field effects in a plasma . . . . . . . . . . . . . 5

1.3.2 Magneto inertial fusion schemes. . . . . . . . . . . . . . 6

1.3.3 Open questions in MagLIF . . . . . . . . . . . . . . 7

1.4 Objectives and structure of the thesis . . . . . . . . . . 8

1.5 Publications . . . . . . . . . . . . . . . . . 9

2 Magnetized plasma expansion into vacuum 11

2.1 General overview . . . . . . . . . . . . . . . . . . . 11

2.2 Statement of the problem . . . . . . . . . . . . 12

2.2.1 Methodology .................. 13

2.3 Results . . . . . . . . . . . . . . . . . . . . 14

3 Plasma-liner interface: mass ablation effects 27

3.1 General overview . . . . . . . . . . . . . . . . . 27

3.2 Statement of the problem . . . . . . . . . . . . . . . 28

3.2.1 Methodology . . . . . . . . . . . . . . . . . . 29

3.3 Results . . . . . . . . . . . . . . . . . . . 31

4 Plasma-liner interface: magnetic pressure effects 47

4.1 General overview . . . . . . . . . . . . . . . . . . 47

4.2 Statement of the problem . . . . . . . . . . . . . . . 48

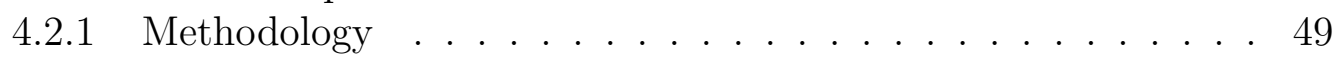

4.3 Results . . . . . . . . . . . . . . . . . 50

5 Plasma-liner interface: mass diffusion and liner material effects $\mathbf{6 3}$

5.1 General overview . . . . . . . . . . . . . . . 63

5.2 Statement of the problem . . . . . . . . . . . . . 64

5.2.1 Methodology .................. 65

5.3 Results . . . . . . . . . . . . . . . . . . . 68 
6 Magnetic flux conservation in an imploding plasma $\quad 89$

6.1 General overview . . . . . . . . . . . . . . . . 89

6.2 Statement of the problem . . . . . . . . . . . . . . 90

6.2.1 Methodology . . . . . . . . . . . . . 92

6.3 Results . . . . . . . . . . . . . . . . . . . 93

7 Alpha heating enhancement in magnetized implosions 103

7.1 General Overview . . . . . . . . . . . . . . . . . . . 103

7.2 Statement of the problem . . . . . . . . . . . . . . . 104

7.2 .1 Methodology . . . . . . . . . . . . 105

7.3 Results . . . . . . . . . . . . . . . . 106

8 Conclusions $\quad 117$

8.1 Future work . . . . . . . . . . . . . . . . . . . . . . 119

$\begin{array}{ll}\text { Bibliography } & 121\end{array}$ 


\section{Introduction}

\subsection{Thermonuclear fusion}

Energy production by fusion reactions is one of the most ambitious projects in Mankind. Controlled thermonuclear fusion is claimed to become a sustainable, clean and limitless energy source that represents an attractive alternative to the current energy production technologies based on thermal and nuclear fission power plants. The fusion reaction most convenient for terrestrial energy production is the deuterium tritium (DT) reaction

$$
\mathrm{D}+\mathrm{T} \rightarrow \alpha(3.5 \mathrm{MeV})+\mathrm{n}(14.1 \mathrm{MeV}),
$$

since it has the largest cross section [6]. Thermonuclear fusion concept is based on the occurrence of copious amount of DT reactions in a plasma of sufficiently high temperature. The alpha particles $\alpha$ distribute their kinetic energy in the plasma by Coulomb collisions, maintaining its high temperature and allowing for more DT reactions to happen in a self-sustained process.

The first interesting question that arises is what temperature should the plasma attain. Of course at such high temperature the plasma would lose its energy in two different ways, conduction and radiation. The latter occurs principally as Bremsstrahlung [41]. If the conduction losses are optimistically neglected in a first instance, one can define the critical temperature [57] or ideal ignition temperature [6] as the temperature at which the internal energy released by fusion products is equal to the radiated power. For a DT plasma, this temperature corresponds to $T_{\mathrm{id}}=4.3 \mathrm{keV}$.

The second question that arises is how to confine matter at such high temperatures. Two main approaches have been developed: magnetic and inertial confinement. The former consists of confining the plasma by means of strong magnetic fields [87, 62], while the latter relies on the confinement exerted by the very mass inertia of the plasma $[59,6]$. Mass inertia keeps the plasma together during the moment that a sound wave needs to travel through it [54]. This short moment, typically one tenth of nanosecond, makes the inertial confinement approach an inherently pulsed process, as compared to the magnetic confinement approach where a steady state is sought.

Attaining the ideal temperature is not the only requirement to ensure the success of a thermonuclear reactor. As stated in the seminal article of Lawson in 1957 
[57], the reaction has to be sustained for a sufficient time. For the DT reaction, a conservative estimation derived by Lawson requires the particle density $n$ times the confinement time $t$ to exceed $n t>10^{14} \mathrm{~s} / \mathrm{cm}^{3}$. For a nanosecond pulse, this yields $n>10^{23} \mathrm{~cm}^{-3}$. Nowadays, particle densities about $n \sim 10^{25} \mathrm{~cm}^{-3}$ are considered in inertial confinement fusion (ICF), which is equal to thousands of times the solid density. Such high density values can be attained by compressing matter by the use of lasers, as initially proposed by Nuckolls and collaborators in 1972 [67].

\subsection{Inertial confinement fusion}

Inertial confinement fusion relies on the compression of small spherical pellets of fuel to produce thermonuclear reactions [17]. The pellet typically consists of a hollow shell capsule made of solid (cryogenic) DT with an outer ablator layer. The capsule is filled with DT vapor in equilibrium with the cryogenic DT. Different types of external radiation sources are employed to drive the implosion. In the so-called direct drive approach [25], a laser directly illuminates the outer surface of the pellet; whereas in the indirect drive approach [60], the laser irradiates a high-Z enclosure (hohlraum) surrounding the target, which emits X-rays that irradiate the capsule.

Whichever the approach is, the source of radiation causes the outer surface of the capsule to ablate, and an ablation pressure is established. A sequence of shock waves are launched inside the shell that merge at the inner surface and break through into the DT vapor. The shell begins to accelerate inwards towards the center, while the shock within the DT vapor moves ahead of it. The shock reaches the center, reflects and attains the converging shell. Soon after, the deceleration phase begins. During this phase, the kinetic energy of the shell is converted into thermal energy and the DT vapor is compressed and heated $[18,13]$. As the temperature increases, the shock waves degenerate into sonic waves, the pressure in the DT vapor becomes homogeneous, and a hot spot is formed - a high-temperature, low density region surrounded by a lower-temperature, higher-density DT shell [25]. The hot spot undergoes an adiabatic implosion before stagnation. Heat flux drives mass ablation off the inner shell surface into the hot spot, and thermal conduction losses are thereby recycled back. At stagnation, the hot spot is expected to attain the ignition temperature and a sufficiently high density so that enough thermonuclear reactions can take place at the center (ignition). A burn wave propagates outwards igniting the remaining fuel, which ultimately expands.

The characteristic target size is of the order of 3 to 5 millimeters. The laser pulse intensity is typically $\sim 10^{15} \mathrm{~W} / \mathrm{cm}^{2}$. An ablation pressure of the order of $\sim 100 \mathrm{Mbar}$ is established. This drives the implosion at a velocity of $V_{i} \sim 300 \mathrm{~km} / \mathrm{s}$ to ensure appropriate hot spot conditions for ignition. 


\subsubsection{Requirements for ignition}

The confinement time has typically been estimated as the ratio between the radius of the hot spot and the speed of sound in the plasma at stagnation. Since the speed of sound depends on the square root of the temperature, the Lawson ignition conditions for $n t$ and $T$ in ICF are more appropriately expressed in terms of areal density of the hot spot $\rho R_{\mathrm{hs}}$ and temperature $T_{\mathrm{hs}}$ at stagnation.

The requirements for $\rho R_{\mathrm{hs}}$ and $T_{\mathrm{hs}}$ can be derived straightforwardly from the energy balance applied to a homogeneous DT plasma requiring that, at stagnation, the energy deposited by the alpha particles overcomes the losses due to conduction and radiation, so the temperature increases. This has been done in detail in Ref. [6]. Among all the possible combinations, the one that requires a minimum energy is chosen, which leads to

$$
\rho R_{\mathrm{hs}}=0.25 \mathrm{~g} / \mathrm{cm}^{2}, \quad T_{\mathrm{hs}}=8 \mathrm{keV} .
$$

However, this simple derivation is rather conservative since it does not take into account the confinement exerted by the surrounding cold shell (tamping effect). It stands for a static criterion in the sense that it is only required that temperature increases at the stagnation moment; no evolution of the explosion is accounted for. A more complete analysis leading to an alternative derivation of a Lawson criterion for ICF has been performed by Betti and collaborators [96, 14, 15, 23]. The authors considered the whole evolution of the target after stagnation, thus in this sense the criterion can be regarded as dynamic. The equations of continuity and energy conservation in the hot spot were solved together with the shell momentum conservation. The ignition condition is seen as a pressure instability, rather than a temperature increase requirement. This instability takes place for a value of the ignition parameter $\chi \equiv \varepsilon_{\alpha} P_{\mathrm{hs}} \tau S\left(T_{\mathrm{hs}}\right) / 24$ larger than unity, where $\varepsilon_{\alpha}$ is the alpha particle birth energy $3.5 \mathrm{MeV}$, and $S\left(T_{\mathrm{hs}}\right)$ is the fusion reactivity $\langle\sigma v\rangle$ weighted with $T^{2}$ and averaged over the hot spot volume. It is intimately related to an equivalent form of the Lawson parameter $P_{\mathrm{hs}} \tau$, with $P_{\mathrm{hs}}$ hot spot pressure at stagnation and $\tau$ the confinement time. The latter arises naturally from the shell momentum equation and is based on the mass inertia of the shell at stagnation $M_{s h}, \tau=\sqrt{M_{s h} / 4 \pi P_{\mathrm{hs}} R_{\mathrm{hs}}}$. Since the shell is typically thin, its mass can be expressed as $M_{s h}=4 \pi \rho \Delta R^{2}$, with $\Delta$ the shell thickness. But the product $\rho \Delta$ can be approximated by the areal density of the target as a whole $\rho R_{\text {tot }}$, and thus, as shown in Refs. [96, 14], the ignition condition $\chi>1$ can be expressed in terms of $\rho R_{\text {tot }}$ and $T_{\mathrm{hs}}$, reading

$$
\rho R_{\mathrm{tot}} T_{\mathrm{hs}}^{2.6}>50 \mathrm{~g} \cdot \mathrm{cm}^{-2} \cdot \mathrm{keV}^{2.6} .
$$

The advantage to express the Lawson criterion in terms of the total areal density is that it can be experimentally measured, since the latter can be obtained through the detection of the spectrum of fusion products, whereas the hot spot temperature can 
be inferred from the Neutron Time-of-Flight diagnostic [96]. Interestingly, Zhou and Betti [95] derived hydrodynamic relations between stagnation and in-flight phases, and obtained

$$
\begin{gathered}
T_{\mathrm{hs}} \sim \alpha_{\mathrm{if}}^{-0.15} V_{i}^{1.25} E_{L}^{0.07}, \\
\rho R_{\mathrm{tot}} \sim \alpha_{\mathrm{if}}^{-0.54} V_{i}^{0.06} E_{L}^{0.33}, \\
\rho R_{\mathrm{hs}} \sim \alpha_{\mathrm{if}}^{-0.55} V_{i}^{0.62} E_{L}^{0.27},
\end{gathered}
$$

with $\alpha_{\text {if }}$ the in-flight adiabat and the $E_{L}$ the laser energy. The hot spot areal density and the total areal density can be thereby related as $\rho R_{\mathrm{hs}} \approx 0.21 \rho R_{\mathrm{tot}}^{0.72} T_{\mathrm{hs}}^{0.46}$, which finally allows to express the dynamic Lawson criterion (1.2.2) in terms of $\rho R_{\mathrm{hs}}$ and $T_{\mathrm{hs}}$,

$$
\rho R_{\mathrm{hs}} T_{\mathrm{hs}}^{1.4}>3.5 \mathrm{~g} \cdot \mathrm{cm}^{-2} \cdot \mathrm{keV}^{1.4} .
$$

It is worth noticing that this ignition requirement is less exigent than Eq. (1.2.1), since it takes into account the tamping effect of the shell. From the expressions (1.2.3), (1.2.5) and (1.2.4), it can be seen that the ignition criterion indirectly sets a minimum value of laser energy and implosion velocity. As suggested before, implosion velocities about $V_{i} \sim 300 \mathrm{~km} / \mathrm{s}$ are required. Implosions in such conditions are susceptible to different sources of instabilities leading to asymmetries that potentially degrade the quality of it, resulting in poor conditions attained at stagnation.

\subsubsection{Implosion asymmetries}

The two major sources of asymmetries are the target-fabrication nonuniformities and the nonuniformities in the driver illumination, known as laser imprint [83, 77, 78]. These asymmetries can give rise to hydrodynamic instabilities that amplify them during the implosion and can even endanger the integrity of the capsule. The main hydrodynamic instabilities developing in ICF experiments are the RichtmyerMeshkov instability [68, 61, 35] and the Rayleigh-Taylor instability [19, 53, 86, 72, $16,76]$.

One of the key points of the success of ICF research lays on the understanding of these instabilities in order to suppress them and ensure a symmetric implosion. It is at this point where the addition of an external magnetic field can be beneficial, since it allows to relax the implosion requirements, which in turn makes the implosion more robust against these instabilities.

\subsection{Magneto inertial fusion}

Magneto inertial fusion (MIF) is an approach that combines the intrinsic features of inertial confinement fusion (heating by compression, pulsed concept) with the 
insulation provided by a magnetic field $B$, characteristic of magnetic confinement fusion [92]. The benefit derived from the introduction of an external magnetic field is the reduction of the implosion requirements, notably the hot spot areal density at stagnation and the implosion velocity.

The relaxation of the implosion requirements can be simply understood based on physical grounds. MIF exploits the insulating properties of the magnetic field. The plasma heat conductivity is significantly reduced [56], which directly lowers the conduction losses during the implosion. Compression heating does not need to overcome these losses, so important in ICF schemes, and the ignition temperature can be attained with a lower implosion velocity. As a consequence, less powerful, more efficient devices could be envisaged to drive the implosion of MIF targets. Furthermore, the magnetic field promotes the fraction of the alpha particle energy deposition in the fuel $f_{\alpha}$, as it forces the alphas to follow helicoidal trajectories rather than straight lines [9]. As a consequence, the areal density of the hot spot, which is directly related to $f_{\alpha}[52]$, is not required to attain such high values. The parameter $B R$, rather than $\rho R$, becomes the figure-of-merit in MIF. And this is one great advantage of this approach: the addition of an external magnetic field allows to explore a wide parameter space, which provides versatility to overcome the difficulties that may arise.

Conceptually, MIF schemes are based on magnetic field amplification based on the Faraday-Lenz's law. When the conductive hot spot is compressed, azimuthal currents are induced so as to increase the magnetic field and conserve the magnetic flux. Consequently, a high degree of plasma magnetization can be achieved at stagnation.

\subsubsection{Magnetic field effects in a plasma}

The presence of a magnetic field can modify the transport properties of a plasma. This occurs when the magnetic field is so intense that the gyroradius of the plasma particles is of the order of their mean free path. Yet, a fully ionized plasma is intrinsically a fluid composed of electrons and ions, which have disparate masses. For this reason, the electrons get magnetized for lower magnetic field intensities compared to ions. Plasma magnetization is therefore measured by the electron Hall parameter $x_{e}$, which stands for the gyrofrequency times the collision time of the electron, $x_{e}=\omega_{e} \tau_{e}$. Equivalently, the magnetization of the ions is given by $x_{i}=\omega_{i} \tau_{i}$. Typically, both parameters are related as $x_{i} \sim x_{e} \sqrt{m_{e} / m_{i}}$, with $m_{e}$ and $m_{i}$ standing for the electron and ion masses, respectively.

Throughout this thesis, the transport properties derived in the popular review by Braginskii [20] are used. Of particular interest to MIF is the thermal conduction suppression in the perpendicular direction to the magnetic field lines. When $x_{e}>1$, the electrons get magnetized and heat is carried by the unmagnetized ions. When the magnetic field is further increased and $x_{i}>1$, which in a deuterium plasma is 
equivalent to $x_{e}>43$, the ions get magnetized and the heat conductivity is strongly suppressed and scales as $x_{e}^{-2}$.

The presence of an external magnetic field also plays a role in the friction forces between electrons and ions. Particularly, a thermal friction force component perpendicular to both the magnetic field lines and the temperature gradients arises. This phenomenon is known as the Nernst effect. It is therefore present in the momentum equation for electrons, which stands for a generalized Ohm's law. It thereby contributes to the electric field dynamics, and ultimately plays a role in the induction equation. Mathematically, the Nernst term appears in the induction equation as a convection term in which the convection velocity is proportional and in the same direction as the heat flux.

Besides modifying the transport properties of a plasma, the magnetic field also affects the dynamics of it through the magnetic pressure and stress tensor. They appear explicitly in the momentum equation, and enrich the nature of the waves that develop in an ideal plasma, that is, in the absence of transport terms. These waves, which in a neutral fluid correspond to sonic waves propagating at the local speed of sound, are classified as fast and slow magnetosonic waves [1]. The former propagates faster than both the local speed of sound and Alfvén velocity, while the latter propagates slower than the minimum of any of those velocities.

\subsubsection{Magneto inertial fusion schemes.}

The first experiments demonstrating ICF neutron yield improvement due to magnetization were reported in 1977 [91]. Some years later, Lindemuth and Kirkpatrick surveyed the parameter space for magnetized fuel targets [58]. They explored a slow velocity $(<1 \mathrm{~cm} / \mu \mathrm{s})$ and low initial density $(\sim 1 \mu \mathrm{g} / \mathrm{cc})$ regime known as magnetized target fusion [50] (MTF). Although this approach could offer a lower cost path to fusion, Jones and Mead [46] showed in their calculations that the magnetic field inhibited the propagation of a burn wave, suggesting that the gain could be limited to 10 .

More recently, magnetized laser-driven high speed implosion experiments have been reported for cylindrical $[37,36,51]$ and spherical [44] geometry. These experiments demonstrated magnetic field compression up to many tens of megagauss, indicating that a significant magnetization of the hot spot can be achieved. Fusion yield enhancement around $30 \%$ in magnetized laser-driven implosions of spherical capsules has also been reported recently [22]. A review of the state of the art in MIF research can be found in Ref. [92].

\subsubsection{Magnetized liner inertial fusion (MagLIF)}

In 2010, Slutz and collaborators [84] proposed a scenario in which a pulsed power device drives the implosion of a cylindrical target with the fuel surrounded by a 
beryllium liner. The target is initially magnetized with $10-30 \mathrm{~T}$, and the fuel is preheated by a laser to temperatures $200-400 \mathrm{eV}$. Fuel preheating is intended to reduce the convergence ratio required to attain the ignition temperature. Implosion velocities, of the order of $10 \mathrm{~cm} / \mu \mathrm{s}$, are higher than the MTF regime but still smaller than conventional ICF implosions. The initial density range is $1-10 \mathrm{mg} / \mathrm{cc}$, while the final areal density is about $0.07 \mathrm{~g} / \mathrm{cm}^{2}$. This scenario, referred to as MagLIF (magnetized liner inertial fusion), appears as a promising way towards fusion energy and is attracting more and more attention. The cylindrical geometry is better suited for the introduction of an axial magnetic field, and the smaller implosion velocity and areal density allow for pulsed power devices such as $Z$ facility in Sandia National Laboratories [69] to drive the implosion, which are more efficient and of a smaller cost than laser-based facilities [92]. The first fully integrated experiments testing the MagLIF concept [34] produced up to $2 \times 10^{12}$ thermonuclear deuterium-deuterium neutrons, proving the potentiality of this concept. In addition, high-gain implosions can be envisaged, overcoming the limitations in gain that the magnetic field imposes to the propagation of the burn wave. In 2012, Slutz and Vesey [85] proposed to add a dense cryogenic deuterium-tritium layer on the inside surface of the metal liner, so most of the fuel remains cold during the compression. The simulations showed that the gain can scale up to 100 for a 60 MA peak current in $Z$, and 1000 for 70 MA, which points out the attractiveness of this scheme.

MagLIF has been scaled down in size to adapt to the laser facility OMEGA at the Laboratory for Laser Energetics (LLE) [26, 8]. OMEGA delivers $\sim 1000 \times$ less energy to a target than $Z$, so the linear dimensions of the target in this laser-driven version of MagLIF are reduced by $\sim 10$. Preliminary results from the first integrated experiments demonstrated a $2 \times$ increase in the neutron yield [8]. There are several motivations to conduct research on laser-driven MagLIF. First, pulsed power devices environments are polluted in terms of electromagnetic noise, making it difficult for diagnostics. Second, OMEGA can perform $10 \times$ more shots per day than $Z$ and therefore provide better statistics. Third, laser-driven MagLIF stands for a different experimental data point which enriches the knowledge about magnetized implosions. Finally, the results will give confidence to extrapolate MagLIF to ignition scale designs.

\subsubsection{Open questions in MagLIF}

Magnetized liner inertial fusion is still at its stage of birth and several fundamental questions related to the magnetohydrodynamics of the implosions remain open. A first issue concerns the dynamics of the corona expansion in the presence of an external magnetic field. This process if of great importance since it sets the ablation pressure level that ultimately drives the implosion. This study becomes specially attractive in MIF spherical targets, where the orientation of the magnetic field lines with respect to the expansion changes with the latitude of the surface point in consideration. 
A particularly interesting feature reported in the MagLIF seminal paper [84] is the degradation of the magnetic field compression caused by the Nernst effect (magnetic field convection in the heat flux direction). The magnetic field value at stagnation is less than expected for a highly conductive hot spot, which endangers the fusion yield and the overall quality of the implosion. It still remains an open question how exactly the Nernst term affects the implosion dynamics and the magnetic flux conservation and what are the mechanisms to reduce its effect. The bulk of this thesis is devoted to this question.

Another pertinent question concerns the effect of the magnetic pressure in the hot spot dynamics. A significant value of magnetic pressure is undesirable since more energy driver would be required for the same hydrodynamic compression. However, by the end of the implosion, the magnetic pressure can be comparable to the hot spot thermal pressure and play a significant role in the dynamics of the stagnation phase. Yet, magnetic pressure effects have typically been disregarded in a MagLIF context.

A similar interest arouses the liner and fuel mixing due to mass diffusion. A specific feature of MagLIF is that the enclosing liner is not made of cryogenic DT. Thus, the material ablated into the hot spot is different from the fuel, which may lead to significant fuel pollution and degrade the implosion performance. Although mass diffusion in multi-component plasmas has gained attention in an ICF context, it still remains unexplored in magnetized implosions.

Finally, a dynamic ignition criterion in the sense commented in Sec. 1.2.1 has not been derived yet for magnetized cylindrical implosions. This issue is of fundamental importance since it provides metrics to quantify the quality of them. Some progress in this subject was made in the static ignition criterion developed in Ref. [9], which shed light on the benefits of the magnetic field on the alpha particles enhancement.

\subsection{Objectives and structure of the thesis}

The main objective of this thesis is to investigate the fundamental magnetohydrodynamics processes appearing in magneto inertial fusion schemes, notably in MagLIF, which aim to answer the open questions outlined in Subsec. 1.3.3. It has been divided into the following objectives:

1. Understand the corona dynamics in magnetized targets with focus on the magnetic field confinement effect in the expansion.

2. Gain insight into the Nernst term on the magnetic flux conservation in a hot magnetized plasma enclosed by a cold liner. Analyze the effect of magnetization on the topology of the flow and on the the thermal losses reduction.

3. Study the magnetic pressure effects in a relevant MagLIF fusion-like plasma. 
4. Understand the liner - fuel mixing due to mass diffusion and evaluate its relevance in a MagLIF context.

5. Analyze the dynamics of a magnetized implosion and the feedback between the magnetic field compression and the evolution of the hydrodynamic variables. Evaluate the Nernst term effect and elucidate how to suppress it.

6. Derive a dynamical ignition criterion for magnetized implosions and evaluate the benefits of the hot spot magnetization at stagnation.

In order to tackle every objective, a set of thoughtful problems has been conceived. They are simple enough to allow for an analytical resolution, but at the same time they capture all the physics realistic to MagLIF. The analysis and resolution of every problem has been thoroughly derived in six articles, each of which deals with one of the aforementioned objectives. Every article is introduced, motivated and summarized in Chapters 2 to 7 of this thesis, respectively, which have been ordered accordingly to the previous enumeration of the objectives. The articles are attached at the end of every chapter. All of the them have been published in international peer-reviewed journals except from the one in Chapter 7, which is expected to be submitted soon. The analysis presented in this last article has been the result of the collaboration with the undergraduate student Jaime Paradela Díaz during his Final Project, supervised by Prof. Francisco Javier Sanz Recio (thesis supervisor) and myself. Finally, overall conclusions and future work are described in Chapter 8.

\subsection{Publications}

1. F. García-Rubio, A. Ruocco and J. Sanz, "Plasma expansion into a vacuum with an arbitrarily oriented external magnetic field", Physics of Plasmas 23, 012103 (2016).

2. F. García-Rubio and J. Sanz, "Mass ablation and magnetic flux losses through a magnetized plasma-liner wall interface", Physics of Plasmas 24, 072710 (2017).

3. F. García-Rubio, J. Sanz and R. Betti, "Magnetic flux conservation in an imploding plasma", Physical Review E 97, 011201(R) (2018).

4. F. García-Rubio and J. Sanz, "Magnetic pressure effects in a plasma-liner interface", Physics of Plasmas 25, 042114 (2018).

5. F. García-Rubio and J. Sanz, "Mass diffusion and liner material effect in a MagLIF fusion-like plasma", Physics of Plasmas 25, 082112 (2018).

6. J. Paradela, F. García-Rubio and J. Sanz, "Alpha Heating Enhancement in MagLIF Targets: An Analytic Model", to be submitted in Physics of Plasmas. 



\section{Magnetized plasma expansion into vacuum}

\subsection{General overview}

In this article, the dynamics of a magnetized plasma expanding into a vacuum is analyzed. The main motivation to perform this study is to gain physical insight into the dynamics of the corona of laser-driven inertial confinement fusion spherical targets embedded in a magnetic field $[22,44]$. Understanding this process is crucial since it establishes the ablation pressure level that drives the compression. In the early stages of the expansion, geometrical factors can be disregarded and the plasma dynamics at the surface of the capsule can be locally modeled as a semi-infinite plasma expanding into a vacuum with an external magnetic field. The orientation of the magnetic field with respect to the expansion direction depends on the latitude of the point of the surface under study, as the magnetic field lines are initially parallel and the expansion is radial, see Fig. 2.1.1.

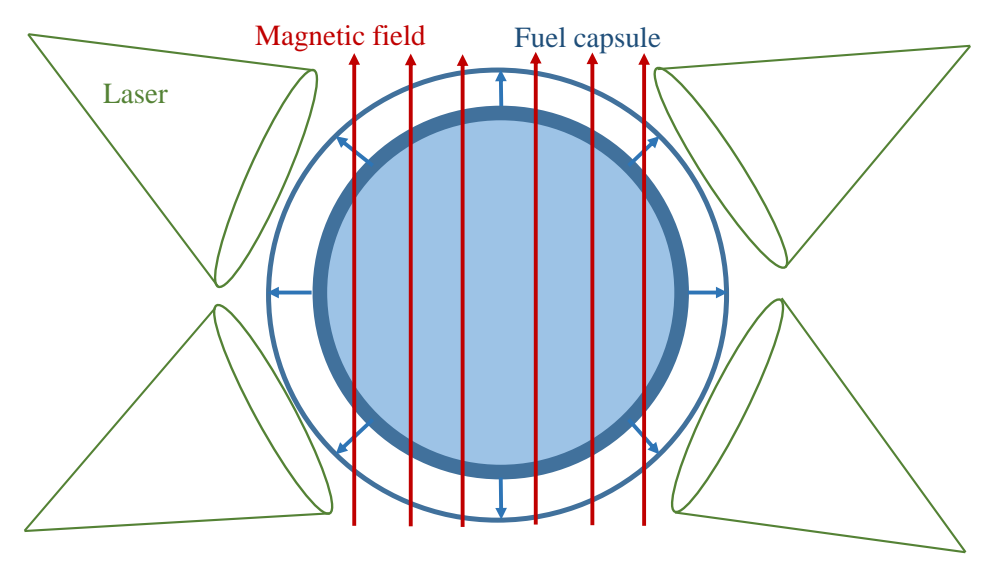

Figure 2.1.1: Scheme of the corona expansion of a spherical capsule embedded in a magnetic field.

Previous work on plasma expansion dates back to 1966 [39], where the self-similar motion of an electron-ion plasma in the absence of collisions and magnetic field was investigated under the framework of the kinetic theory. More recently, charge separation effects in an unmagnetized plasma expansion without collisions have been 
studied in Ref. [65]. The effect of the magnetic field on the plasma expansion dynamics has been investigated within the framework of ideal magneto-hydrodynamics (MHD) in Refs. [5, 45, 12]. In these articles, only the case of perpendicular magnetic field orientation with respect to the expansion was analyzed. Two configurations were treated: magnetized plasma expanding into an unmagnetized vacuum and unmagnetized plasma expanding into a magnetized vacuum.

In magneto inertial fusion schemes, the magnetic field encloses the capsule and penetrates into it. Therefore, a magnetized plasma expanding into a magnetized vacuum corresponds to a more realistic picture of the corona dynamics. This is the configuration investigated in this work, which has not been reported before. The initial orientation of the magnetic field is left as a free parameter, and both the perpendicular and oblique cases are treated separately. The latter case is analyzed for the first time in the literature.

\subsection{Statement of the problem}

The evolution of a semi-infinite plasma initially at rest and expanding into a vacuum with a preimposed external magnetic field in planar geometry has been thoroughly studied. An artistic scheme of the problem is shown in Fig. 1 of the attached article. The plasma is treated under the hypotheses of ideal MHD fluid model [55]. The magnetic field is initially uniform, imposed both in the plasma and in the vacuum, and its initial inclination with respect to the expansion direction is $\alpha$.

The qualitative description of the plasma motion goes as follows. Due to the absence of mechanical equilibrium, the plasma expands against the magnetic field in the vacuum. The problem only depends on time $t$ and streamwise direction $x$. The expansion takes place through a rarefaction wave where plasma density decreases. The foot of this wave penetrates into the unperturbed plasma, transmitting the information of the presence of the vacuum. The magnetic field in the vacuum exerts a confinement effect: the magnetic pressure opposes to the expansion. Consequently, the plasma is piled up at the end of the rarefaction wave, presenting a well defined expansion front. The magnetic field in the plasma evolves frozen into it.

When the magnetic field initial inclination is perpendicular, $\alpha=\pi / 2$, it keeps this inclination for all time. The frozen-in condition establishes that the magnetic field is proportional to the plasma density, hence it decreases in the rarefaction wave. According to Ampère's law, an electric current distribution in the direction perpendicular to both the expansion and the magnetic field develops to support the gradients of the latter. The vacuum is reached with a finite plasma density, and the magnetic field presents a jump at this interface. Consequently, a strong electric current opposite to the previous distribution takes place at this interface.

When the magnetic field initial inclination is oblique, $\alpha<\pi / 2$, its evolution is relatively more complex since the magnetic field lines are stretched due to the plasma 
motion. The presence of a streamwise component of the magnetic field induces a Lorentz force in the axis perpendicular to both the expansion direction and the electric current, and triggers plasma motion in this axis. This streamwise component allows for a second kind of rarefaction wave to take place, where the plasma expands smoothly until the vacuum is reached with zero density.

\subsubsection{Methodology}

The motion of the plasma is studied under the ideal MHD assumptions, and planar geometry is assumed. The governing equations stand for mass, momentum and entropy conservation together with Faraday's and Gauss's laws, Eqs. (6) - (10) in the article. They form a system of hyperbolic differential equations for density, velocity and magnetic field.

The proposed problem lacks a characteristic length, and it admits a self-similar solution in the variable $x / t$. This means that the dependent variables (density, magnetic field and velocity) do not depend on $x$ and $t$ independently, but in that specific combination of them [7]. Density and magnetic field are normalized with their value at the plasma at rest, velocity is normalized with the speed of sound of the plasma at rest, $c_{0}$, and the self-similar coordinate $\xi=x / c_{0} t$ is introduced. The system of governing equations is thereby reduced to the system of ordinary differential equations, Eq. (13) in the paper. The only two governing parameters appearing on it are the initial inclination of the magnetic field lines $\alpha$ and the thermal-to-magnetic pressure ratio $\beta$. The latter can also be expressed in terms of the speed of sound $c_{0}$ and the Alfvén velocity of the unperturbed plasma $v_{A 0}$ as $\beta=2\left(c_{0} / v_{A 0}\right)^{2}$.

The system of equations is homogeneous in the derivatives of the variables. Therefore, it admits the trivial solution denoted as plateau, where all the variables are kept constant. Additionally to it, non-trivial solutions can be found by imposing linear dependence among the equations of the system. This condition yields a relationship between the dependent variables called dispersion relation, Eq. (17) in the article. Physically, it correspond to non-linear waves traveling in the plasma. The solution of the problem is therefore obtained by assembling different branches of plateaus and non-linear waves in order to satisfy the boundary conditions. The match between the branches is performed through weak discontinuities, allowed in hyperbolic systems.

The frontier with the vacuum is treated as a fluid surface where conservation of momentum through the interface is imposed, Eqs. (11) and (12) in the article. It deserves special attention since the structure of the interface changes depending on $\alpha$. For perpendicular magnetic field, $\alpha=\pi / 2$, a discontinuity in both density and magnetic field is allowed, and the magnetic pressure in the vacuum counterbalances the thermal plus magnetic pressures in the plasma. However, for $\alpha<\pi / 2$, the 
magnetic field must be continuous, and the plasma needs to expand until its density drops to zero.

The matching has performed done using numerical methods. However, a complete analytic solution has been derived for the specific value of the adiabatic index $\gamma=2$, described in the article.

\subsection{Results}

When the magnetic field is perpendicular, the dispersion relation represents a nonlinear magnetosonic wave that propagates, relative to surfaces of constant density, faster than both the local speed of sound and the Alfvén velocity, Eq. (18) in the article. It stands for a rarefaction wave in the sense that plasma density decreases while it expands. The magnetic field is reduced along with the expansion, and it generates a favorable magnetic pressure gradient that enhances it. Consequently, the foot of the rarefaction wave travels into the unperturbed plasma faster than the speed of sound, the latter being the rarefaction wave speed in a neutral fluid expansion, described in Ref. [54]. The structure of the magnetosonic wave does not satisfy the boundary conditions at the frontier with the vacuum at its edge. Therefore, a plateau takes place connecting both structures. Physically, this plateau represents the aforementioned pile-up of plasma at the edge of the expansion. Hence, the magnetic field tends to enhance the expansion due to the favorable magnetic pressure gradient inside the plasma but, at the same time, it confines it as a result of the magnetic pressure at the border, which acts as if it was a piston. The total effect results in an effective confinement, and the frontier with the vacuum is placed before with respect to its position in a neutral fluid expansion. The structure of the expansion when the magnetic field is perpendicular can be seen in Fig. 2 in the article.

When the magnetic field is oblique, the dispersion relation yields two kind of waves: fast and slow magnetosonic waves, Eq. (22). The first propagates, with respect to surfaces of constant density, faster than both the speed of sound and the Alfvén velocity, and the second propagates slower than any of them. A complete description of magnetosonic waves in a plasma can be found in Ref. [1]. The two solutions represent rarefaction waves where plasma density decreases. The unperturbed plasma expands through the fast magnetosonic wave. Then it is piled up in a plateau after which it expands again smoothly through a slow magnetosonic wave to reach the conditions in the vacuum: zero density and initial magnetic field value. In the latter region, the magnetic pressure gradient is adverse, hence it also exerts a net confinement effect opposing the expansion as in the perpendicular case. The structure of the expansion with an oblique magnetic field is shown in Fig. 5 in the article.

The whole parameter space $0 \leq \alpha \leq \pi / 2,0<\beta<\infty$ has been explored. Particularly, the study made for oblique magnetic field when $\alpha \ll 1$ and $\pi / 2-\alpha \ll 1$ shows 
that the solution consistently retrieves the neutral fluid and perpendicular magnetic field cases, respectively. Asymptotic results have been derived in the limits of a weak and strong magnetic field, $\beta \gg 1$ and $\beta \ll 1$ in the perpendicular case, which support the qualitative description of the solution presented. 



\title{
Plasma expansion into a vacuum with an arbitrarily oriented external magnetic field
}

\author{
F. García-Rubio, ${ }^{1, a)}$ A. Ruocco, ${ }^{2}$ and J. Sanz ${ }^{1}$ \\ ${ }^{1}$ E.T.S.I. Aeronáutica y del Espacio, Universidad Politécnica de Madrid, Madrid 28040, Spain \\ ${ }^{2}$ Universitá degli studi di Napoli Federico II, 80138 Napoli, Italy
}

(Received 30 October 2015; accepted 21 December 2015; published online 6 January 2016)

\begin{abstract}
Plasma expansion into a vacuum with an external magnetic field is studied under the ideal magnetohydrodynamic hypothesis. The inclination of the magnetic field with respect to the expansion direction is arbitrary, and both the perpendicular and the oblique cases are separately analyzed. A self-similar solution satisfying the boundary conditions is obtained. The interface with the vacuum is treated as a fluid surface, and jump conditions concerning the momentum conservation are imposed. The effect of the intensity of the magnetic field and its inclination is thoroughly studied, and the consistency of the solution for small and large inclinations is investigated. (C) 2016 AIP Publishing LLC. [http://dx.doi.org/10.1063/1.4939476]
\end{abstract}

\section{INTRODUCTION}

The features of an expanding plasma with an external magnetic field are of great relevance for the understanding of laser-matter interaction processes. In Inertial Confinement Fusion (ICF), a high energy laser or ion beam heats a Deuterium-Tritium (DT) hollow micro-capsule. ${ }^{1}$ The outer part of the shell ablates and expands forming the corona. The ablation pressure at the corona pushes the capsule radially inwards and it implodes reaching Lawson fusion conditions at the core of the target (hot spot).

Magneto Inertial Fusion $(\mathrm{MIF})^{2}$ is an interesting approach to ICF that allows to relax the requirements to attain ignition conditions. It consists of magnetizing the fusion chamber, so that the corona formation and the implosion occur embedded in an external magnetic field. The electron thermal conductivity is reduced and, consequently, ignition temperatures can be reached at the hot spot with smaller implosion velocities. ${ }^{3}$

A great part of the research on MIF concerns the experimental characterization of the magnetized target compression, with the focus on the measure and understanding the increase in the magnetic flux intensity. ${ }^{4}$ However, the analysis of the corona expansion in a magnetized environment, a key to establish the ablation pressure level that rules this compression, has been given less attention.

In the early stages of the expansion, geometrical factors can be disregarded and the plasma dynamics at the surface of the capsule can be locally modeled as a semi-infinite plasma expanding into a vacuum with an external magnetic field. The orientation of the magnetic field with respect to the expansion direction depends on the latitude of the point of the surface under study, as the magnetic lines are initially parallel and the expansion is radial. Plasma expansion into a vacuum has been investigated applying kinetic theory, ${ }^{5}$ and phenomena as important as initial charge separation at the frontier with the vacuum were analyzed. More recently, the

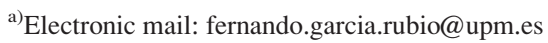

effects of a perpendicular magnetic field have been studied within the framework of ideal magneto-hydrodynamics (MHD) with applications to ICF. ${ }^{6-8}$ Anderson et al. ${ }^{6}$ analyzed both the expansion of a magnetized plasma into unmagnetized vacuum and an unmagnetized plasma expanding against a magnetic field. In the latter, the authors suggested that the jump conditions at the boundary with the vacuum are not compatible with the self-similar solution satisfying the governing equations. Instead, a pile up of plasma was proposed to form behind the frontier, which had been experimentally observed by Tonon et al. ${ }^{9}$ for the same initial configuration. Johnson and Lonngreen ${ }^{8}$ investigated the dynamics of a magnetized plasma expanding into an unmagnetized vacuum by means of a Lagrangian representation. In a more recent paper, Bennaceur-Doumaz and Djebli ${ }^{7}$ analyzed in detail the density and temperature profiles of an initially magnetized laser produced plasma expansion and their sensitivity to the thermal/magnetic pressure ratio.

The two initial configurations treated in the papers previously mentioned have a discontinuity in the magnetic field at the border with the vacuum. According to Ampère's law, this jump has to be supported by a strong current localized in the plasma-vacuum interface. In our opinion, an initial configuration where both the plasma and the vacuum are equally magnetized is a more realistic picture for typical MIF experiments. Besides, no initial confined current density distribution is initially required, which describes a more natural situation.

The understanding of plasma expansion dynamics is also of great importance for astrophysics. Plechaty et al. ${ }^{10}$ performed some experimental analysis of the laser-produced plasma expansion in a perpendicular magnetic field with applications to this field, whereas Nersisyan et al. ${ }^{11}$ describes a spherical plasma expansion into unmagnetized vacuum.

The present work aims at analyzing theoretically the dynamics of a semi-infinite region of magnetized plasma expanding into a magnetized vacuum. Plasma is treated under the hypothesis of ideal MHD fluid model (for MHD applicability, see discussion in Refs. 12 and 13), and the 
Euler equations with the electromagnetic terms are fully solved and discussed. The interface with the vacuum is treated as a fluid surface and the conservation of momentum has to be satisfied through it. The case where the magnetic field is oblique with respect to the expansion, not seen treated in the literature to our knowledge, is also solved. The ideas exposed in this work allow to overcome some of the incompatibilities reported in the literature previously mentioned.

The paper is organized as follows: in Sec. II, the governing equations and the boundary conditions are described. The self-similar character of the motion is outlined. In Sec. III, the case where the magnetic field is perpendicular to the expansion is investigated. In Sec. IV, the expansion with an oblique magnetic field is thoroughly studied, and the asymptotic limit to both parallel and perpendicular magnetic field is retrieved. In Sec. V, conclusions are drawn.

\section{GOVERNING EQUATIONS AND BOUNDARY CONDITIONS}

We consider at $t=0$ a plasma medium at rest, with density $\rho_{0}$ and thermal pressure $p_{0}$, occupying the half space $x<0$, and we let it expand into the vacuum at $t>0$. A uniform magnetic field $\vec{B}_{0}=\left\{B_{0} \cos \alpha, 0, B_{0} \sin \alpha\right\}$ is initially imposed both in the plasma and in the vacuum. The angle $\alpha$ is the initial inclination of the magnetic field with respect to the expansion direction (see schematic Fig. 1). We describe the dynamics of the expansion under the ideal MHD hypothesis, and the plasma is a perfect gas. In this context, the equations governing the expansion are

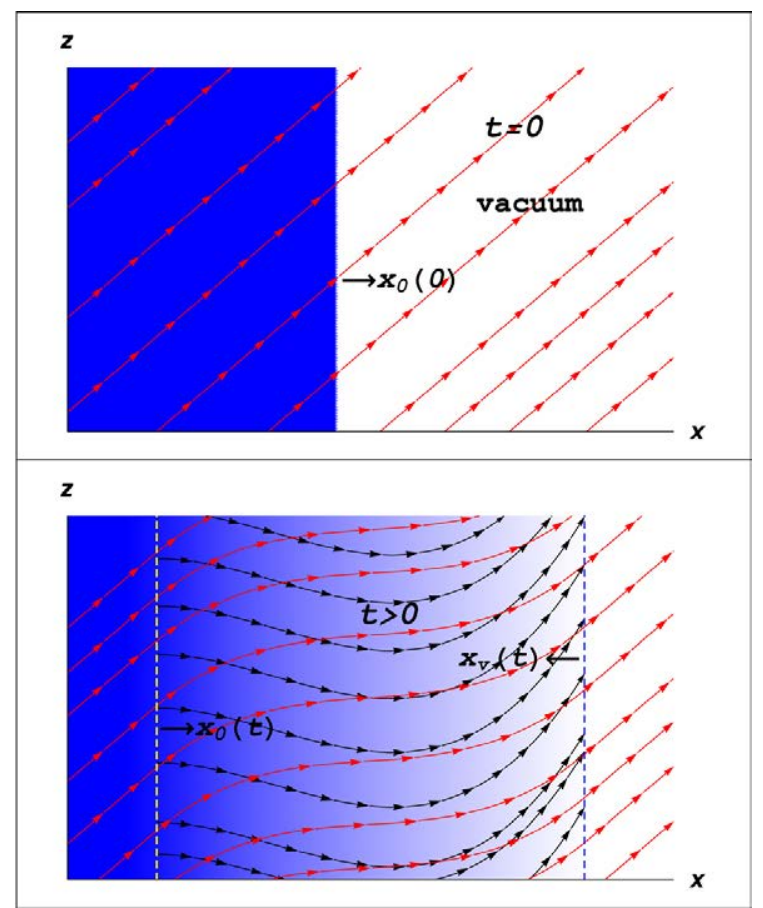

FIG. 1. Artistic scheme of a plasma expansion. The solid block in blue represents the plasma medium. The color gradation represents density. Arrows in red: magnetic field lines. Arrows in black: flow streamlines.

$$
\begin{gathered}
\frac{\partial \rho}{\partial t}+\nabla \cdot(\rho \vec{V})=0 \\
\rho \frac{\partial \vec{V}}{\partial t}+\rho \vec{V} \cdot \nabla \vec{V}+\frac{1}{\mu_{0}} \vec{B} \times(\nabla \times \vec{B})+\nabla p=0 \\
p=p_{0}\left(\frac{\rho}{\rho_{0}}\right)^{\gamma} \\
\frac{\partial \vec{B}}{\partial t}-\nabla \times(\vec{V} \times \vec{B})=0 \\
\nabla \cdot \vec{B}=0 .
\end{gathered}
$$

Here, $\rho, \vec{V}, p$, and $\vec{B}$ are the density, velocity, pressure, and magnetic field, respectively; $\mu_{0}$ is the magnetic permeability of the vacuum; and $\gamma$ is the adiabatic exponent. Equations (1)-(3) stand for mass, momentum, and entropy conservation, respectively; Eq. (4) refers to the Faraday law with ideal MHD hypothesis; and Eq. (5) stands for the solenoidal character of $\vec{B}$. As a result of the ideal MHD equations and the geometry of the problem, both the magnetic field and flow velocity lie in the $x z$-plane and every magnitude depends on $x$ and $t$ only. Hence, Equations (1)-(5) read

$$
\begin{gathered}
\frac{\partial \rho}{\partial t}+\frac{\partial\left(\rho V_{x}\right)}{\partial x}=0 \\
\frac{\partial V_{x}}{\partial t}+V_{x} \frac{\partial V_{x}}{\partial x}+\frac{B_{z}}{\mu_{0} \rho} \frac{\partial B_{z}}{\partial x}+\frac{p_{0}}{\rho} \frac{\partial}{\partial x}\left(\frac{\rho}{\rho_{0}}\right)^{\gamma}=0 \\
\frac{\partial V_{z}}{\partial t}+V_{x} \frac{\partial V_{z}}{\partial x}-\frac{B_{x}}{\mu_{0} \rho} \frac{\partial B_{z}}{\partial x}=0 \\
\frac{\partial B_{z}}{\partial t}-\frac{\partial}{\partial x}\left(V_{z} B_{x}-V_{x} B_{z}\right)=0 \\
B_{x}=B_{0} \cos \alpha
\end{gathered}
$$

As boundary conditions, we impose that our physical system is not perturbed at $x= \pm \infty$. Here, we anticipate the existence of two frontiers at $x=x_{0}(t)$ and $x=x_{v}(t)$. The first one corresponds to the foot of a magneto-sonic wave traveling towards the $x<0$ region with constant velocity, and none perturbation exists in $x<x_{0}(t)$. Such a frontier is a weak discontinuity. The second one corresponds to the interface between the plasma and the vacuum moving towards the $x>0$ region at constant velocity too. In this frontier, the particles travel along with the interface velocity (fluid surface condition). Mathematically, this statement imposes $V_{x}\left(t, x=x_{v}\right)=\mathrm{d} x_{v}(t) / \mathrm{d} t$. Beyond $x_{v}(t)$, the magnetic field remains constant and equal to the initial magnetic field. Therefore, conservation of momentum in $x$ and $z$ at the plasma-vacuum boundary yields, respectively

$$
\begin{gathered}
p_{0}\left(\frac{\rho\left(t, x_{v}\right)}{\rho_{0}}\right)^{\gamma}+\frac{B_{z}^{2}\left(t, x_{v}\right)}{2 \mu_{0}}=\frac{B_{0}^{2} \sin ^{2} \alpha}{2 \mu_{0}}, \\
B_{0} \cos \alpha B_{z}\left(t, x_{v}\right)=B_{0}^{2} \cos \alpha \sin \alpha .
\end{gathered}
$$

Notice that the structure of the discontinuity at the vacuum interface when the magnetic field is perpendicular $(\alpha=\pi / 2)$ differs significantly from the structure for other 
values of the initial inclination $\alpha$. For $\alpha=\pi / 2$, Eq. (12) is automatically satisfied, so a jump in $B_{z}$ is allowed through the interface and Eq. (11) allows for the existence of a density discontinuity. However, for $\alpha \neq \pi / 2, B_{z}$ has to be continuous through the interface, and density has to drop to zero to balance $x$-momentum flux. In this case, the frontier plasmavacuum is a weak discontinuity.

\section{A. Self-similarity and dimensionless equations}

Since the governing equations [(6)-(12)] and the initial conditions lack a characteristic length, they admit a selfsimilar solution in the variable $\mathrm{x} / \mathrm{t} .{ }^{14}$ Hence, we introduce the self-similar coordinate $\xi=x / c_{0} t$, where $c_{0} \equiv \sqrt{\gamma p_{0} / \rho_{0}}$ is the speed of sound in the plasma at rest, and the dimensionless self-similar MHD variables are in the form: $n(\xi)$ $=\rho / \rho_{0}, u(\xi)=V_{x} / c_{0}, w(\xi)=V_{z} / c_{0}, \quad$ and $\quad b_{z}(\xi)=B_{z} / B_{0}$. The system [(6)-(9)] is reduced to the following ordinary differential equations system:

$$
\begin{gathered}
(u-\xi) n^{\prime}+n u^{\prime}=0, \\
n(u-\xi) u^{\prime}+n^{\gamma-1} n^{\prime}+\frac{\left(b_{z}^{2}\right)^{\prime}}{\beta}=0, \\
n(u-\xi) w^{\prime}-\frac{2 \cos \alpha}{\beta} b_{z}^{\prime}=0, \\
(u-\xi) b_{z}^{\prime}+b_{z} u^{\prime}-w^{\prime} \cos \alpha=0,
\end{gathered}
$$

where the prime denotes derivative with respect to $\xi$. The boundary conditions (11) and (12) become

$$
\begin{gathered}
\frac{n\left(\xi_{v}\right)^{\gamma}}{\gamma}+\frac{b_{z}\left(\xi_{v}\right)^{2}-\sin ^{2} \alpha}{\beta}=0, \\
\cos \alpha\left(b_{z}\left(\xi_{v}\right)-\sin \alpha\right)=0 .
\end{gathered}
$$

Here, $\xi_{v}$ is the self-similar coordinate of the plasma-vacuum frontier, which is an eigenvalue of the problem, and then $x_{v}(t)=\xi_{v} c_{0} t$. The fluid surface condition imposes $u\left(\xi_{v}\right)=\xi_{v}$. The only two governing parameters in Eqs. (13)-(15) are the initial inclination of the magnetic field lines $\alpha$ and $\beta$, which is $\gamma$ times the ratio between the initial thermal and the magnetic pressures, and can also be expressed in terms of the speed of sound $c_{0}$ and the Alfvén velocity $v_{A 0}$ in the unperturbed plasma

$$
\beta=2 \gamma \mu_{0} p_{0} / B_{0}^{2}=2\left(c_{0} / v_{A 0}\right)^{2} .
$$

Since the system of Equation (13) is homogeneous in the derivatives of the variables, it always admits the trivial solution $\left\{n, u, w, b_{z}\right\}=$ const, denoted as plateaus. Besides, imposing linear dependence among the equations of the system yields non-trivial solutions. This requirement implies setting to zero the determinant of the matrix formed by the coefficients of the variables with derivatives. This condition is called hereinafter dispersion relation, which yields

$$
\begin{aligned}
\frac{n}{2}(u-\xi)^{4} & -\left(\frac{\cos ^{2} \alpha+b_{z}^{2}}{\beta}+\frac{n^{\gamma}}{2}\right)(u-\xi)^{2} \\
& +\frac{n^{\gamma-1}}{\beta} \cos ^{2} \alpha=0 .
\end{aligned}
$$

Non-trivial solutions correspond to non-linear waves traveling in a plasma, ${ }^{13}$ the structure of which is determined by (17) and three independent equations of (13). Their physical meaning is explained in detail in Secs. III and IV. In the case of a standard fluid expansion into vacuum without magnetic field, the dispersion relation yields a sonic wave that propagates at the local speed of sound $c:(u-\xi)^{2}=\left(c / c_{0}\right)^{2}$.

The solution satisfying the boundary conditions is built by piecewise assembling different branches of plateaus and non-linear waves, following the same resolution as classical self-similar problems in fluid mechanics. ${ }^{14}$ We require therefore that a wave connects with the plasma at rest at its foot, denoted as $\xi_{0}=x_{0} /\left(c_{0} t\right)$, which is also an eigenvalue of the problem. Another branch, either a plateau or a wave, must connect at $\xi_{v}$ with the boundary with the vacuum. These two branches as well as any additional inner branch have to match through weak discontinuities, which are allowed in hyperbolic systems.

\section{PERPENDICULAR MAGNETIC FIELD}

When the magnetic field is perpendicular to the expansion $(\alpha=\pi / 2)$, the dispersion relation (17) reads

$$
(u-\xi)^{2}=n^{\gamma-1}+2 \frac{b_{z}^{2}}{n \beta} .
$$

The physical meaning of the dispersion relation gets clearer when it is expressed in terms of the local speed of sound $c=c_{0} n^{(\gamma-1) / 2}$ and the local Alfvén velocity $v_{A}=v_{A 0} b_{z} /$ $\sqrt{n}=c_{0} b_{z} \sqrt{2 /(n \beta)}$

$$
(u-\xi)^{2}=\left(\frac{c}{c_{0}}\right)^{2}+\left(\frac{v_{A}}{c_{0}}\right)^{2} .
$$

It represents therefore a non-linear magnetosonic wave that propagates, relative to surfaces of constant density, faster than both the local speed of sound and the Alfvén velocity. The unperturbed plasma state $\left(u=0, n=b_{z}=1\right)$ can be retrieved within the structure of this wave, and Eq. (18) particularized at this state yields the velocity of the foot of it

$$
\xi_{0}=-\sqrt{1+\frac{2}{\beta}} \equiv-\sqrt{1+\left(\frac{v_{A 0}}{c_{0}}\right)^{2}} .
$$

In the derivation of this expression, a sign choice due to the squared term in the left hand side of (18) has been made. It corresponds to the election of the expansion direction. As we consider that the plasma expands towards positive $x$, we choose the sign plus. The frontier with the vacuum, on the contrary, cannot be reached by the magnetosonic wave, since the dispersion relation (18) is not compatible with the fluid surface condition $u\left(\xi_{v}\right)=\xi_{v}$ together with the streamwise momentum conservation Eq. (14). This means that the border with the vacuum has to be retrieved by means of a plateau. This situation differs from a standard fluid expansion, where the sonic wave retrieves on itself the conditions at the vacuum. 
The evolution of $u, b_{z}$, and $w$ in the magnetosonic wave as a function of the density $n$ is given by the system (13) setting $\alpha=\pi / 2$. First, it can be seen that there is no transverse motion triggered, since the Lorentz force only acts on the expansion direction, hence $w=0$. Combining the continuity and Faraday equations, we easily derive the frozen-in condition, and the equation of momentum allows to obtain the streamwise velocity $u$

$$
\begin{gathered}
b_{z}=n \\
u=\int_{n}^{1} \sqrt{x^{\gamma-3}+\frac{2}{x \beta}} \mathrm{d} x .
\end{gathered}
$$

Finally, the structure of the magnetosonic wave is completed by the dispersion relation Eq. (18), which expresses the relation between $n$ and $\xi$.

As stated before, the border with the vacuum has to be reached by a plateau. This plateau has to match with the magnetosonic wave at its foot $\xi=\xi_{1}$, and at the same time satisfy the conservation of momentum and the fluid surface conditions at its end $\xi=\xi_{v}$. Let the sub-index $p$ refers to the plasma state at the plateau. The matching with the nonlinear wave through a weak discontinuity imposes that $b_{z p}=n_{p}$. The value of the density is then given by the jump condition (14), while the foot and the end of the plateau are obtained from the fluid surface condition and the dispersion relation, respectively

$$
\begin{aligned}
& n_{p}^{\gamma}=\frac{\gamma}{\beta}\left(1-n_{p}^{2}\right), \quad b_{z p}=n_{p}, \\
& u_{p}=\int_{n_{p}}^{1} \sqrt{x^{\gamma-3}+\frac{2}{x \beta}} \mathrm{d} x, \\
& \xi_{v}=u_{p}, \quad \xi_{1}=\xi_{v}-\sqrt{n_{p}^{\gamma-1}+\frac{2 n_{p}}{\beta}} .
\end{aligned}
$$

The calculations are greatly simplified for $\gamma=2$, and analytical expressions can be obtained. The streamwise velocity in the magnetosonic wave as a function of density reads

$$
u=2 \sqrt{1+\frac{2}{\beta}}(1-\sqrt{n}) .
$$

The plasma state at the plateau, its foot, and its end are

$$
\begin{aligned}
& n_{p}=b_{z p}=\sqrt{\frac{2 / \beta}{1+2 / \beta}}, \\
& u_{p}=\xi_{v}=2 \sqrt{1+2 / \beta}\left(1-\sqrt[4]{\frac{2 / \beta}{1+2 / \beta}}\right), \\
& \xi_{1}=2 \sqrt{1+2 / \beta}\left(1-\frac{3}{2} \sqrt[4]{\frac{2 / \beta}{1+2 / \beta}}\right) .
\end{aligned}
$$

The mathematical model derived also includes the case of an isothermal plasma expansion. The isothermal condition is retrieved from the entropy conservation (3) by setting $\gamma=1$. Contrary to an isothermal neutral fluid expansion, where $\xi_{v}=2 /(\gamma-1) \rightarrow \infty$, the plasma does not fill all the space for $t>0$ because the magnetic field in the vacuum acts as a piston opposing the expansion. In this case, the density at the interface with the vacuum yields $n_{p}=\left(\sqrt{\beta^{2}+4}\right.$ $-\beta) / 2$, whereas the velocity of the frontier with the vacuum is finite and reads $\xi_{v} \approx \ln \beta$ for large values of $\beta$ (weak magnetic field).

The solution for a general $\gamma$ has to be integrated by numerical methods. We have chosen to present the case $\gamma=5 / 3$ as it corresponds to a monoatomic gas whose microscopic kinematics have three degrees of freedom. In Fig. 2, plasma expansion for $\beta=5$ and $\gamma=5 / 3$ is depicted, and it can be seen that it does not differ qualitatively from the analytic solution just obtained for $\gamma=2$. The magnetosonic wave can be seen as a rarefaction wave where plasma expands decreasing its density. As a result of the frozen-in condition, the magnetic field value also diminishes in this region. It can be noticed that the magnetic pressure term $B_{z} \partial B_{z} / \partial x$ plays the role of a favorable pressure gradient, enhancing therefore the expansion. For this reason, the foot of the rarefaction wave penetrates into the plasma at rest faster than the speed of sound. The streamwise velocity increases due to both the Lorentz force and the thermal pressure gradient. A plateau where all the variables keep a constant value follows the magnetosonic wave. It represents the pile-up of plasma region mentioned in Ref. 6 and observed experimentally in Ref. 9 In this region, plasma flows at constant velocity until it encounters the border with the vacuum where the fluid surface condition is satisfied. Physically, the plateau is originated as a consequence of the confinement exerted by the magnetic pressure in the vacuum. Hence, the magnetic field tends to enhance the expansion in the

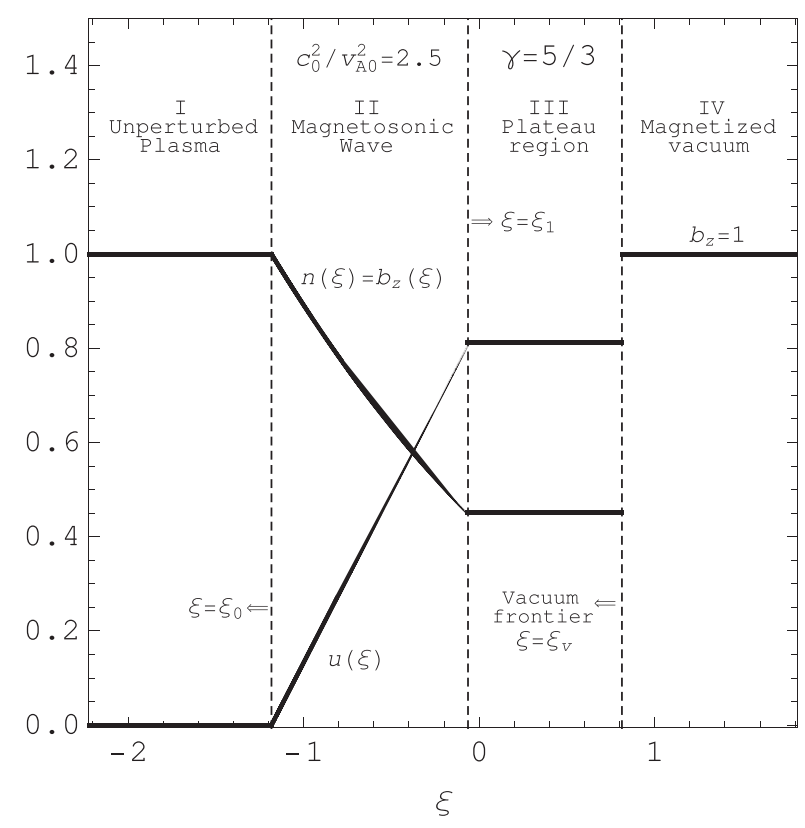

FIG. 2. Self-similar density $n$, streamwise velocity $u$, and transverse magnetic field component $b_{z}$ dimensionless profiles in a plasma expansion with perpendicular magnetic field. $\beta=5$. 
magnetosonic wave but, at the same time, it confines the plasma due to the magnetic pressure at the border. The total effect results in an effective confinement as the frontier is placed before with respect to its position in the standard fluid expansion: $\xi_{v}<2 /(\gamma-1)$; that is, the magnetic field would act like a piston placed at the end of the expansion. It can be seen that some of the inconsistencies reported in the literature are therefore overcome, since a complete self-similar solution satisfying all the boundary conditions exists.

Following Ampère's Law, $\nabla \times \vec{B}=\mu_{0} \vec{j}$, an electric current $\vec{j}$ must support a magnetic field gradient. Therefore, an electric current volumetric distribution along the $y$-axis, pointing towards the reader in Fig. 2, fills the magnetosonic region. Besides, a strong surface distribution supporting the jump in the magnetic field is concentrated in the frontier with the vacuum. It lies in the same axis as the electric current distribution in the magnetosonic region but it points towards the opposite direction. Both distributions have the same integral.

\section{A. Effect of the intensity of the magnetic field}

The effect of the magnetic field intensity is shown in Figs. 3 and 4. It can be seen that both the height of the discontinuity and the width of the plateau increase for higher intensities. At the same time, the position of the frontier with the vacuum steps back as a consequence of the magnetic confinement. On the other hand, the standard fluid expansion is retrieved for low intensities: the discontinuity vanishes, the perturbation wave travels at the speed of sound, and the frontier with the vacuum recovers the classical value $\xi_{v}=2 /(\gamma-1)$.

The scaling laws for the density at the plateau and the velocities of the foot of the rarefaction wave and the interface with the vacuum when the magnetic field is very strong $(\beta \ll 1)$ are

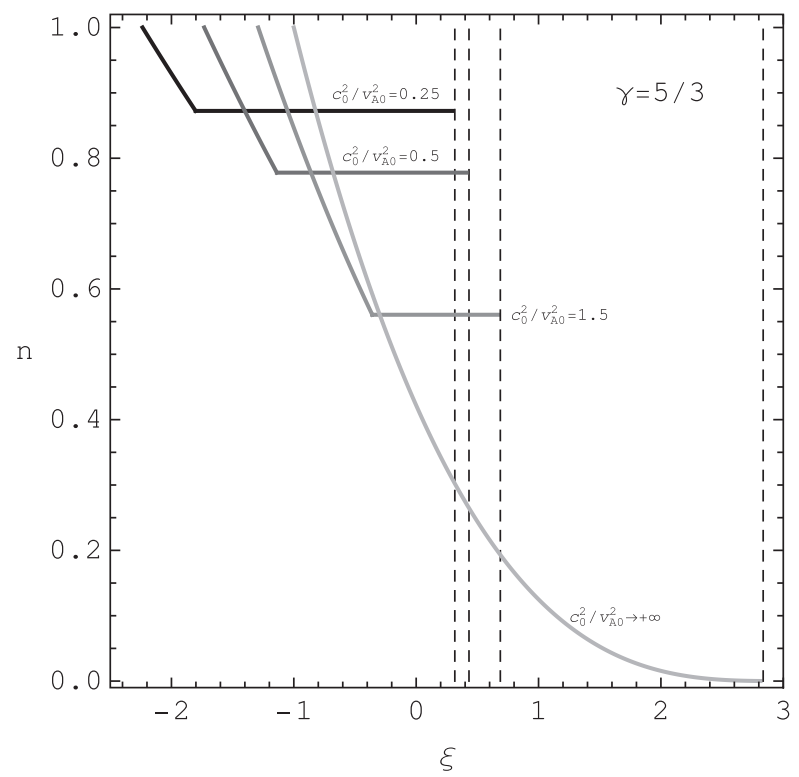

FIG. 3. Self-similar density $n$ profile for different values of $\beta=2 c_{0}^{2} / v_{A 0}^{2}$ in a plasma expansion with perpendicular magnetic field.

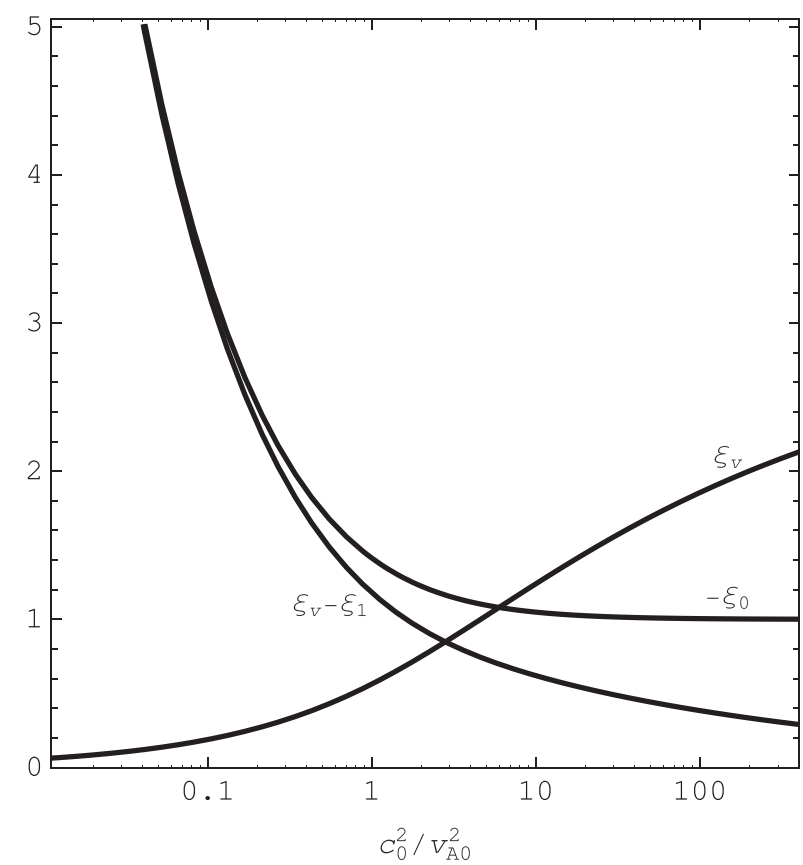

FIG. 4. Velocity of the vacuum frontier $\xi_{v}$, foot of the rarefaction wave $-\xi_{0}$, and self-similar width of the plateau for different values of $\beta=2 c_{0}^{2} / v_{A 0}^{2}$.

$$
\begin{aligned}
& n_{p}=1-\frac{\beta}{\gamma}+O\left(\beta^{2}\right), \\
& \xi_{0}=-\sqrt{\frac{2}{\beta}}+O\left(\beta^{1 / 2}\right), \\
& \xi_{v}=\frac{1}{\gamma} \sqrt{\frac{\beta}{2}}+O\left(\beta^{3 / 2}\right),
\end{aligned}
$$

while the scaling laws for very weak magnetic field $(\beta \gg 1)$ yield

$$
\begin{aligned}
& n_{p}=\left(\frac{\gamma}{\beta}\right)^{1 / \gamma}+O\left(\beta^{-3 / \gamma}\right), \\
& \xi_{0}=-1-\frac{1}{\beta}+O\left(\beta^{-2}\right), \\
& \xi_{v}=\frac{2}{\gamma-1}\left[1-\left(\frac{\gamma}{\beta}\right)^{(\gamma-1) /(2 \gamma)}\right]+O\left(\beta^{-1}\right) .
\end{aligned}
$$

\section{OBLIQUE MAGNETIC FIELD}

The dispersion relation (17) for a general initial inclination $\alpha$ yields

$$
\begin{aligned}
(u-\xi)^{2}= & \frac{2 b_{z}^{2}+n^{\gamma} \beta+2 \cos ^{2} \alpha}{2 n \beta} \\
& \pm \frac{\sqrt{\left(2 b_{z}^{2}+n^{\gamma} \beta+2 \cos ^{2} \alpha\right)^{2}-8 n^{\gamma} \beta \cos ^{2} \alpha}}{2 n \beta}
\end{aligned}
$$

which can be rewritten in terms of the speed of sound and Alfvén velocity as 


$$
\begin{aligned}
(u-\xi)^{2}= & \frac{1}{2}\left(\frac{c}{c_{0}}\right)^{2}+\frac{1}{2}\left(\frac{v_{A}}{c_{0}}\right)^{2} \\
& \pm \frac{1}{2} \sqrt{\left[\left(\frac{c}{c_{0}}\right)^{2}+\left(\frac{v_{A}}{c_{0}}\right)^{2}\right]^{2}-4\left(\frac{c}{c_{0}}\right)^{2}\left(\frac{v_{A}}{c_{0}}\right)^{2} \cos ^{2} \theta}
\end{aligned}
$$

where the angle $\theta$ stands for the inclination of the magnetic field at each location in the expansion, $\theta(\xi)=\arctan \left(B_{z}(\xi) / B_{x}\right)$.

The presence of a magnetic field component parallel to the expansion allows for the appearance of two types of waves as stated in Eq. (22). The plus sign refers to fast magnetosonic waves, whereas the solution with minus stands for slow magnetosonic waves. ${ }^{13}$ The first propagate, with respect to surfaces of constant density, faster than $\max \left(c, v_{A}\right)$, while the latter propagate slower than the $\min \left(c, v_{A}\right)$. The two solutions represent rarefaction waves where density decreases and the streamwise velocity increases.

A second double sign appears due to the squared term in the left hand side of Eq. (22), which corresponds to the pointing of the expansion direction and the position of the unperturbed plasma with respect to the origin. Following the same reasoning as in the perpendicular case, the sign plus is chosen.

The solution satisfying the boundary conditions consists then of branches of plateaus, fast and slow magnetosonic waves. The region connecting with the plasma at rest has to be a fast magnetosonic branch in order to ensure a regular convergence towards the perpendicular magnetic field solution when $\alpha \rightarrow \pi / 2$. On the other hand, it can be seen that the conditions at border with the vacuum $\left(n\left(\xi_{v}\right)=0, b_{z}\left(\xi_{v}\right)\right.$ $=\sin \alpha, u\left(\xi_{v}\right)=\xi_{v}$ ) are compatible with the structure of a slow magnetosonic wave. Consequently, a matching of the fast wave coming from the unperturbed plasma and the slow wave expanding until the vacuum has to be performed once the structure of the waves is obtained.

For this purpose, it is convenient to reformulate the problem by selecting $v=u-\xi$ as the independent variable. With this choice, the two types of waves are treated under the same formulation. The dispersion relation is therefore rewritten as

$$
b_{z}^{2}=\left(1-\frac{n^{\gamma-1}}{v^{2}}\right)\left(\frac{\beta n}{2} v^{2}-\cos ^{2} \alpha\right) .
$$

The governing system of Equation (13) reduces then to direct integration, whereas the dependence with the initial variable $\xi$ is recovered from the streamwise momentum equation

$$
\begin{gathered}
\frac{\mathrm{d} n}{\mathrm{~d} v}=2 \frac{n}{v} \frac{\frac{\cos ^{2} \alpha n^{\gamma-2}}{\beta v^{2}}-\frac{v^{2}}{2}}{(\gamma-1) \frac{\cos ^{2} \alpha n^{\gamma-2}}{\beta v^{2}}-\frac{v^{2}}{2}+n^{\gamma-1}\left(1-\frac{\gamma}{2}\right)}, \\
\frac{\mathrm{d} w}{\mathrm{~d} v}=\frac{\cos \alpha}{n b_{z} v \beta} \frac{\mathrm{d} b_{z}^{2}}{\mathrm{~d} v},
\end{gathered}
$$

$$
\frac{\mathrm{d} v}{\mathrm{~d} \xi}=-\frac{n^{\gamma-2}\left[n\left(1-\frac{\gamma}{2}\right)+\frac{\cos ^{2} \alpha(\gamma-1)}{\beta v^{2}}\right]-\frac{v^{2}}{2}}{n^{\gamma-2}\left[n\left(1-\frac{\gamma}{2}\right)+\frac{\cos ^{2} \alpha(\gamma+1)}{\beta v^{2}}\right]-\frac{3}{2} v^{2}} .
$$

The structure of the waves is therefore given by Eqs. (23)-(26).

Analyzing the particular case $\gamma=2$ can provide insight into this system, since it is greatly simplified. A straightforward analytic integration yields

$$
\begin{gathered}
n=\left(\frac{v}{\mathcal{C}}\right)^{2}, \\
b_{z}^{2}=\left(1-\frac{1}{\mathcal{C}^{2}}\right)\left(\frac{\beta}{2 \mathcal{C}^{2}} v^{4}-\cos ^{2} \alpha\right),
\end{gathered}
$$

where $\mathcal{C}$ is a constant of integration used to match the waves with the boundary conditions. We analyze first the fast magnetosonic wave $(f)$ connecting with the plasma at rest. The conditions $n\left(v=-\xi_{0}\right)=1$ and $b_{z}\left(v=-\xi_{0}\right)=\sin \alpha$ fix the constant $\mathcal{C}_{f}=-\xi_{0}$ and the value for $\xi_{0}$

$$
\xi_{0}=-\sqrt{\frac{1+2 / \beta+\sqrt{(1+2 / \beta)^{2}-8 \cos ^{2} \alpha / \beta}}{2}} .
$$

This expression for $\xi_{0}$ is also valid for a general $\gamma$. It is interesting to notice that $\left|\xi_{0}\right| \geq 1$, hence the foot of the rarefaction wave travels faster or equal to the speed of sound for all angles $\alpha$.

The slow magnetosonic wave $(s)$, on the other hand, connects with the vacuum at $v=0$. The recovery of the magnetic field condition $b_{z}(v=0)=\sin \alpha$ yields $\mathcal{C}_{s}=\cos \alpha$.

The most notorious difference in the structure of the fast and the slow magnetosonic waves lies in the growth of $b_{z}$. Effectively, taking the derivative with respect to $\xi$ in Eq. (28) shows that $\mathrm{d} b_{z} / \mathrm{d} \xi$ is negative for the fast magnetosonic wave; that is, the magnetic field decreases when we move away from the origin of the expansion; whereas $\mathrm{d} b_{z} / \mathrm{d} \xi$ is positive for the slow magnetosonic wave and $b_{z}$ increases until it reaches the value at the vacuum. As a requirement to support the magnetic field gradient, an electric current volumetric distribution pointing towards the positive $y$-axis in the fast magnetosonic wave and towards the negative $y$-axis in the slow wave takes place. Its interaction with the parallel magnetic field $B_{x}$ originates a Lorentz force in the $z$-axis, negative on the fast wave and positive on the slow. Consequently, the transverse motion $w$ is inverted on each wave.

We proceed to match the two magnetosonic waves. We naturally look first for a matching performed through weak discontinuities. However, we cannot connect directly the two branches as we have to impose continuity on $n, u$, and $b_{z}$, and we would only have two free parameters: the position of the point where the nonlinear waves connect and the position of the frontier with the vacuum. A third branch has to be added to build a continuous solution. This branch cannot be 
a nonlinear wave, since it would be indistinguishable from one of the unconnected waves, and the matching problem would remain. It has to be a plateau what connects the fast and slow branches. We have then three free parameters: the front and the end of the plateau and the position of the interface with the vacuum.

Let the sub-indexes 1 and 2 stand for the front and the end of the inner plateau, respectively. The values $v_{1}$ and $v_{2}$ for a general $\gamma$ are obtained from the matching

$$
n_{f}\left(v_{1}\right)=n_{s}\left(v_{2}\right), \quad b_{z f}\left(v_{1}\right)=b_{z s}\left(v_{2}\right)
$$

Solving this system for the case $\gamma=2$ yields

$$
\begin{aligned}
& v_{1}=-\xi_{0}\left(\frac{2}{\beta \xi_{0}^{2}}\right)^{1 / 4}, \\
& v_{2}=\cos \alpha\left(\frac{2}{\beta \xi_{0}^{2}}\right)^{1 / 4} .
\end{aligned}
$$

Continuity in the streamwise velocity allows to obtain the position of the frontier with the vacuum

$$
\xi_{v}=2\left(\xi_{0}+\cos \alpha\right)\left(\frac{2}{\beta \xi_{0}^{2}}\right)^{1 / 4}-2 \xi_{0}
$$

which is smaller than the corresponding value for a standard fluid expansion, confirming that the net effect of an oblique external magnetic field is also to confine the expansion. Finally, the position of the inner plateau reads

$$
\begin{gathered}
\xi_{1}=3 \xi_{0}\left(\frac{2}{\beta \xi_{0}^{2}}\right)^{1 / 4}-2 \xi_{0} \\
\xi_{2}=\left(2 \xi_{0}-\cos \alpha\right)\left(\frac{2}{\beta \xi_{0}^{2}}\right)^{1 / 4}-2 \xi_{0} .
\end{gathered}
$$

An isothermal plasma expansion with an oblique external magnetic field can also be studied within the assumptions of this model by setting $\gamma=1$. In this case, the dispersion relation (23) applied at the frontier with the vacuum, where $n\left(\xi_{v}\right)=0, b_{z}\left(\xi_{v}\right)=\sin \alpha$, yields $v\left(\xi_{v}\right)=\cos \alpha$. This may lead to consider that the frontier with the vacuum is no longer a fluid surface apparently, which also happens in the isothermal standard fluid expansion, where $v\left(\xi_{v}\right)=1$. The integration of Eq. (26) gives $\xi_{v} \rightarrow \infty$. Then, equal to the fluid expansion, the plasma fills all the space for $t>0$, and the concept of frontier with the vacuum loses its proper physical meaning. (We refer to Mora ${ }^{15}$ for more details on isothermal plasma expansion without magnetic field.) The density profile for large values of $\xi$ yields asymptotically $n \sim \exp (-\xi / \cos \alpha)$, consistent with the standard fluid case $n=\exp (-\xi-1)$. The positions of the front and the end of the inner plateau, obtained from the matching condition (30), are of order unity. For $\gamma \neq 1$, but $|\gamma-1| \ll 1$, the profile $n(v)$ is extended for $v<\cos \alpha$, and the frontier with the vacuum is again recovered at $v=0, n(v=0)=0$. Asymptotic analysis shows that the interface with the vacuum travels at $\xi_{v} \approx 2 \cos \alpha /(\gamma-1)$, whereas the profile $n(\xi)$ also decreases exponentially for large $\xi$.

Plasma expansion for $\gamma=5 / 3$ has to be integrated by numerical methods, but it does not differ qualitatively from the analytic solution derived for $\gamma=2$. The results from the numerical integration for $\gamma=5 / 3, \beta=5$, and $\alpha=\pi / 4$ are depicted in Fig. 5. A fast magnetosonic wave enters the plasma at rest (region I) transmitting the presence of the vacuum at a speed greater than the speed of sound. Both the density and the magnetic field decrease, while the plasma expands (region II). This wave is followed by an inner plateau (region III) where the plasma variables remain constant. The end of the inner plateau connects with a slow magnetosonic branch (region IV) where the magnetic field increases while the plasma continues expanding until its density drops to zero at the frontier with the vacuum (region V). The streamwise velocity monotonically increases on each wave, whereas the transverse velocity growth rate changes sign.

It is interesting to compare Figs. 2 and 5, which stand for expansions with the same magnetic field but tilted $45^{\circ}$. The parallel component of the magnetic field originates a Lorentz's force component in the $z$-axis, which triggers motion in this direction ( $w \neq 0$ in Fig. 5). The discontinuity in the frontier plasma-vacuum in the perpendicular case has been replaced by a smooth region where a slow magnetosonic wave takes place. Both structures play the same role in compressing the plasma and confining the expansion through an adverse magnetic pressure gradient. The surface electric current distribution localized in the frontier plasma-vacuum in the perpendicular case is spread out over the whole slow magnetosonic branch in the oblique case.

Finally, the influence of the magnetic field intensity and its initial inclination on the rarefaction wave front velocity $\left|\xi_{0}\right|$ is depicted in Fig. 6. As mentioned before, the

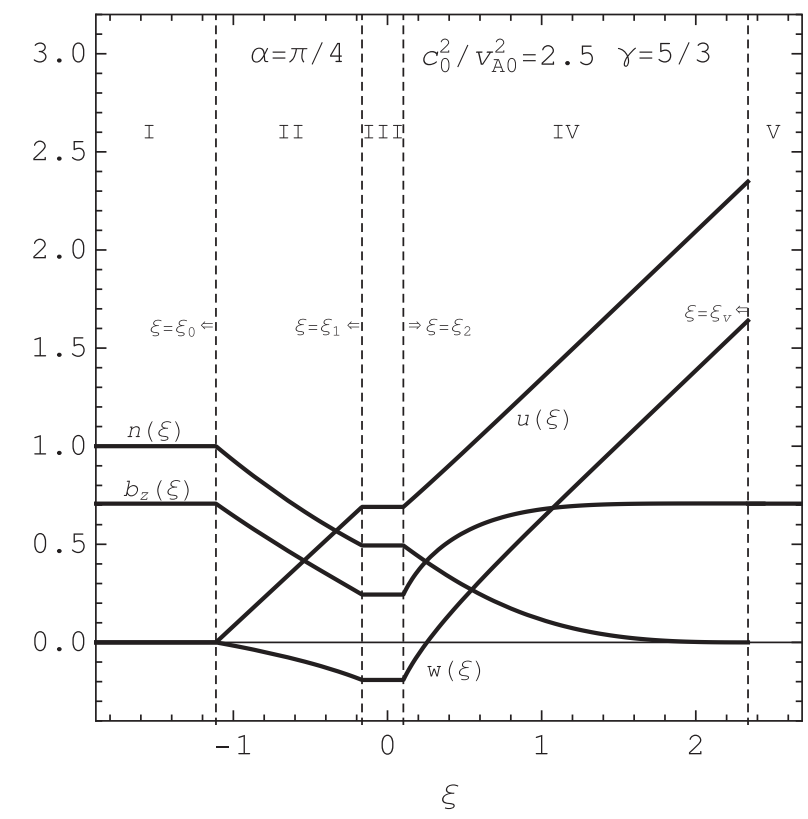

FIG. 5. Self-similar density $n$, streamwise velocity $u$, transverse velocity $w$, and transverse magnetic field component $b_{z}$ dimensionless profiles in a plasma expansion with oblique magnetic field. $\alpha=\pi / 4$ and $\beta=5$. 


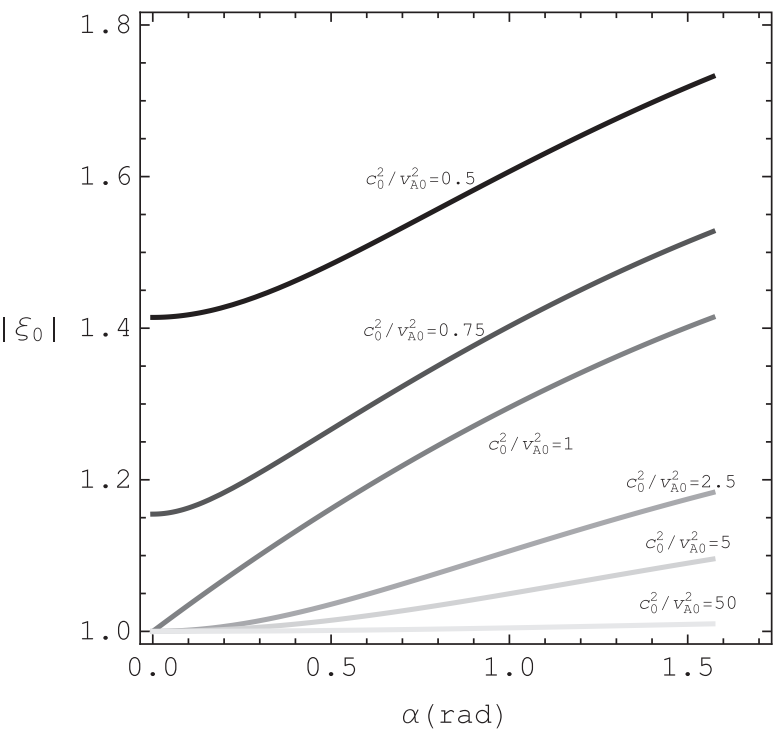

FIG. 6. Velocity of the front of the rarefaction wave $\left|\xi_{0}\right|$ vs. $\alpha$ for different values of $\beta=2 c_{0}^{2} / v_{A 0}^{2}$.

rarefaction wave penetrates faster into the unperturbed plasma for higher values of the magnetic field. The more this field is tilted towards the expansion direction the less the expansion is enhanced. This effect may be relevant in the corona expansion in a homogeneously magnetized MIF chamber, since the velocity of the rarefaction wave depends on the latitude of each point in the outer shell. One would expect to recover $\left|\xi_{0}\right|=1$ for $\alpha \rightarrow 0$ independently of $\beta$. However, it can be seen in Fig. 6 that this is not the case for sufficiently high magnetic field intensities $(\beta<2)$. Thus, it is interesting to test whether the oblique solution can degenerate into the perpendicular solution and the standard fluid expansion when $\alpha$ approaches $\pi / 2$ and 0 , respectively.

\section{A. Recovery of the perpendicular magnetic field expansion}

Plasma expansion for an almost perpendicular initial magnetic field is plotted in Fig. 7. The width of the slow magnetosonic wave shrinks for $\alpha$ close to $\pi / 2$. It is reasonable to interpret that the discontinuity at the frontier plasmavacuum in the perpendicular case is smoothed out into a sharp layer when the magnetic field tilts a little. This sharp layer gradually widens for decreasing $\alpha$.

If $\gamma=2$, the width of the slow magnetosonic wave is

$$
\xi_{v}-\xi_{2}=3 \cos \alpha\left(\frac{2}{\beta \xi_{0}^{2}}\right)^{1 / 4},
$$

where $\xi_{0}$ is given in Eq. (29). Let $\alpha=\pi / 2-\eta$, with $|\eta| \ll 1$. From Eq. (31), we notice that the width of the layer, in terms of the self-similar variable $\xi$, is of order $O\left(\eta^{2}\right)$. An asymptotic analysis around $\alpha=\pi / 2$ performed in the system of equations for a general $\gamma$ shows that the width of the slow magnetosonic wave in this case is also of order $O\left(\eta^{2}\right)$.

We conclude then that although the jump conditions (11)-(12) yield different structures for the perpendicular and

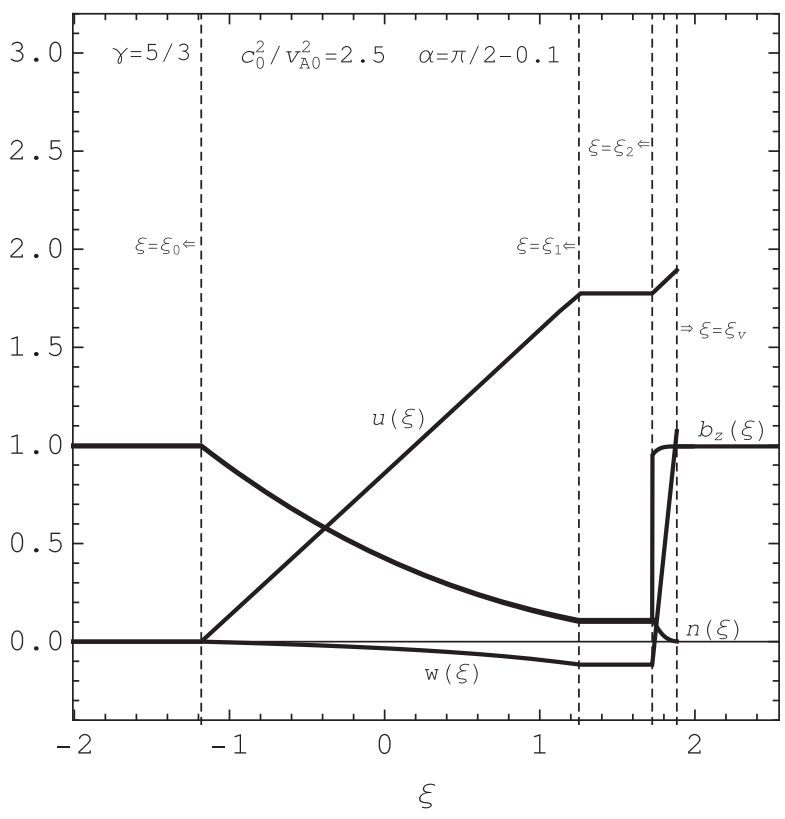

FIG. 7. Self-similar density $n$, streamwise velocity $u$, transverse velocity $w$, and transverse magnetic field component $b_{z}$ dimensionless profiles in a plasma expansion with an almost perpendicular magnetic field. $\alpha=\pi / 2-0.1$ and $\beta=5$.

oblique cases, there is a smooth and consistent passage to the limit when the magnetic field inclination approaches perpendicularity.

\section{B. Recovery of the standard fluid expansion}

A magnetic field completely parallel to the expansion does not affect the plasma dynamics, so the standard fluid expansion solution should be retrieved for $\alpha \rightarrow 0$. In Fig. 8, plasma expansion for a small $\alpha$ and $\beta>2$ is plotted. It can be

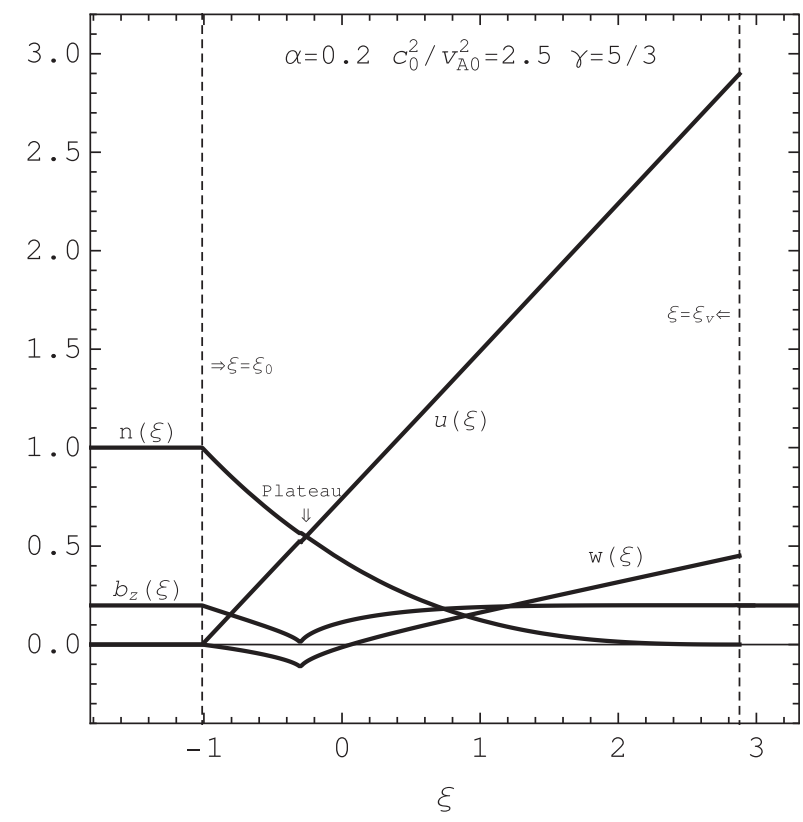

FIG. 8. Self-similar density $n$, streamwise velocity $u$, transverse velocity $w$, and transverse magnetic field component $b_{z}$ dimensionless profiles in a plasma expansion with an almost parallel magnetic field. $\alpha=0.2$ and $\beta=5$. 
seen that the size of the inner plateau shrinks to zero, the front wave velocity tends to one, and the frontier with the vacuum reaches the limit $\xi_{v}=2 /(\gamma-1)$. The fast and slow magnetosonic waves degenerate into a nonlinear sonic wave.

The picture is quite different for a more intense magnetic field $(\beta<2)$, such as the expansion plotted in Fig. 9. The size of the inner plateau no longer diminishes, and it is the fast magnetosonic wave width what shrinks to zero while it penetrates deep into the unperturbed plasma at a velocity higher than the speed of sound. The slow magnetosonic wave, on the contrary, widens and completely degenerates into the sonic wave. Although classic standard fluid expansion is recovered, the plasma at rest is first perturbed by a wave of infinitesimal amplitude.

The threshold which differentiates the two pictures is $\beta^{*}=2$. Effectively, taking the limit $\alpha \rightarrow 0$ in Eq. (29) yields

$$
\lim _{\alpha \rightarrow 0}\left|\xi_{0}\right|= \begin{cases}1 & \text { if } \quad \beta>2 \\ \sqrt{2 / \beta}=\frac{v_{A 0}}{c_{0}} & \text { if } \quad \beta<2 .\end{cases}
$$

The small amplitude wave in Fig. 9 propagates then at the Alfvén velocity $v_{A 0}=B_{0} / \sqrt{\mu_{0} \rho_{0}}$ asymptotically. The underlying physical phenomenon corresponds to the different degeneration of the fast magnetosonic wave on each case. In a plasma expansion with parallel magnetic field, three nonlinear modes can be excited: a sonic wave and two Alfvén waves, each one relating velocity and magnetic field components on an axis perpendicular to the expansion. The motion on each direction is uncoupled, and each mode needs to be specifically excited to develop. When the magnetic field is oblique, the sound wave and the Alfvén wave on the $z$-axis are coupled and form the fast and the slow magnetosonic waves. When the speed of sound is greater than the Alfvén velocity $(\beta>2)$, the two magnetosonic branches

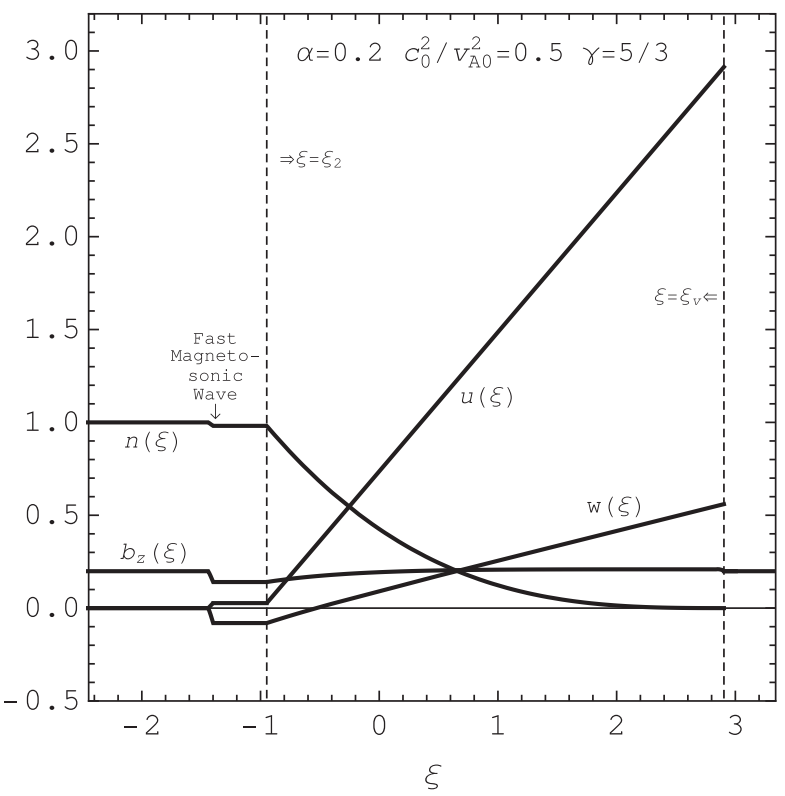

FIG. 9. Self-similar density $n$, streamwise velocity $u$, transverse velocity $w$, and transverse magnetic field component $b_{z}$ dimensionless profiles in a plasma expansion with an almost parallel magnetic field. $\alpha=0.2$ and $\beta=1$. degenerate into pieces of sonic wave. However, when the Afvén velocity is greater than the speed of sound $(\beta<2)$, the fast magnetosonic branch degenerates into an Alfvén wave mode. For small $\alpha$, this mode mainly involves the velocity and the magnetic field perpendicular to the expansion, but it is weakly coupled and can slightly excite motion in the streamwise direction, resulting into a wave of small amplitude.

\section{CONCLUSIONS}

In this paper, plasma expansion into vacuum with an arbitrarily oriented external magnetic field has been solved. A complete analytic solution has been obtained for $\gamma=2$. The expansion is a self-similar process whose structure presents clearly distinguishable regions. These regions correspond to the solutions of the ideal MHD equations: nonlinear waves and plateaus. They are arranged to satisfy the boundary conditions and matched through weak discontinuities. The frontier with the vacuum is treated as a fluid surface where conservation of momentum through the interface is imposed.

Plasma expansion with a perpendicular magnetic field presents four different regions: a first region where the plasma remains unperturbed and at rest is followed by a magnetosonic rarefaction wave where it expands. A plateau comes at the end of the wave in which the plasma is piled up as a consequence of the magnetic confinement. The structure of the jump conditions in the vacuum interface allows for a discontinuity in the magnetic field and plasma density at the end of the plateau. Finally, the magnetic field remains constant in the vacuum. In a plasma expansion with oblique magnetic field, this discontinuity cannot exist and it is replaced by a smooth rarefaction wave. Two types of rarefaction waves take place then: a fast magnetosonic through which the plasma at rest expands until the inner plateau, and the already mentioned slow magnetosonic wave that connects the inner plateau with the vacuum in a continuous way and where density drops to zero. In both configurations, the net effect of the external magnetic field results in a confinement, similar to a piston placed at the end of the expansion.

The effects of the intensity of the magnetic field and its inclination have been analyzed, and a smooth degeneracy when the inclination tends to zero and $\pi / 2$ is observed.

Future work implies studying the stability and characterizing the thickness of the interface discontinuity in the perpendicular case for a finite magnetic Reynolds number as well as comparison with experiments to test the validity of the assumed hypothesis.

\section{ACKNOWLEDGMENTS}

This research was supported by the Spanish Ministerio de Economía y Competitividad, Project No. ENE201454960R.

${ }^{1} \mathrm{~S}$. Atzeni and J. Meyer-ter-Vehn, The Physics of Inertial Fusion (Oxford Science Publications, 2004).

${ }^{2}$ O. V. Gotchev, N. W. Jang, J. P. Knauer, M. D. Barbero, R. Bett, C. K. Li, and R. D. Petraso, "Magento-inertial approach to direct-drive laser fusion,” J. Fusion Energy 27, 25-31 (2008). 
${ }^{3}$ M. R. Gomez, S. A. Slutz, A. B. Sefkow, D. B. Sinars, K. D. Hahn, S. B. Hansen, E. C. Harding, P. F. Knapp, P. F. Schmit, C. A. Jennings, T. J. Awe, M. Geissel, D. C. Rovang, G. A. Chandler, G. W. Cooper, M. E. Cuneo, A. J. Harvey-Thompson, M. C. Herrmann, M. H. Hess, O. Johns, D. C. Lamppa, M. R. Martin, R. D. McBride, K. J. Peterson, J. L. Porter, G. K. Robertson, G. A. Rochau, C. L. Ruiz, M. E. Savage, I. C. Smith, W. A. Stygar, and R. A. Vesey, "Experimental demonstration of fusionrelevant conditions in magnetized linear inertial fusion," Phys. Rev. Lett. 113, 155003 (2014).

${ }^{4}$ M. Hohenberger, P.-Y. Chang, G. Fiksel, J. P. Knauer, R. Betti, F. J Marshall, D. D. Meyerhofer, F. H. Séguin, and R. D. Petrasso, "Inertial confinement fusion implosions with imposed magnetic field compression using the omega laser," Phys. Plasmas 19, 056306 (2012).

${ }^{5}$ A. V. Gurevich, L. V. Pariiskaya, and L. P. Pitaevskii, "Self-similar motion of rarefied plasma," Sov. Phys. JETP 22, 449-454 (1966) .

${ }^{6} \mathrm{D}$. Anderson, M. Bonnedal, and M. Lisak, "Effects of magnetic field of self-similar plasma expansion into vacuum," Phys. Scr. 22, 507-509 (1980).
${ }^{7}$ D. Bennaceur-Doumaz and M. Djebli, "Effect of transverse magnetic field on laser produced plasma expansion into vacuum," Phys. Plasmas 18, 084507 (2011).

${ }^{8}$ S. F. Johnson and K. E. Lonngren, "On the expansion of a magnetized plasma," Phys. Scr. 25, 583-586 (1982).

${ }^{9}$ G. Tonon, M. Rabeau, and D. Shirman, Colloques 32(C5), C5b-139 (1971).

${ }^{10}$ C. Plechaty, R. Presura, S. Wright, S. Neff, and A. Haboub, "Penetration of plasma across a magnetic field,” Astrophys. Space Sci. 322, 195-199 (2009).

${ }^{11}$ H. B. Nersisyan, K. A. Sargsyan, D. A. Osipyan, M. V. Sargsyan, and H H. Matevosyan, "Self-similar analytical model of plasma expansion in a magnetic field,” Phys. Scr. 84, 065003 (2011).

${ }^{12}$ L. D. Landau and E. M. Lifshitz, Electrodynamics of Continuous Media (Pergamon Press, 1960).

${ }^{13}$ A. I. Akhiezer, I. A. Akhiezer, R. V. Polovin, and D. ter Haar, Plasma Electrodynamics: Linear Theory (Pergamon Press, 1975).

${ }^{14}$ L. D. Landau and E. M. Lifshitz, Fluid Mechanics (Pergamon Press, 1959).

${ }^{15}$ P. Mora, "Plasma expansion into a vacuum," Phys. Rev. Lett. 90, 185002 (2003). 


\section{Plasma-liner interface: mass ablation effects}

\subsection{General overview}

In this article, the evolution of a hot magnetized plasma in contact with a cold, dense, unmagnetized plasma is analyzed. The motivation to perform this study lies in the characterization of thermal energy and magnetic flux losses in magnetized implosions. The hot plasma would crudely represent the hot spot whereas the cold plasma would represent the liner.

An important feature of magnetized implosions is the long time that the hot plasma and the cold liner stay in contact compared to classic inertial confinement fusion (ICF) schemes. An analysis of the heat and magnetic flux losses through the plasmaliner interface becomes thereby crucial to improve the design of magnetized capsule implosions. As a first step to perform such analysis, the implosion dynamics have been disregarded in the article presented in this chapter, and only plasma motion triggered by transport processes is considered. Magnetic flux conservation taking into account implosion dynamics are analyzed in the article presented in Chapter 6.

The analysis of magnetic insulation of wall confined plasmas dates back to the work of Alfvén and Smårs in 1960 [2]. Some years later, self-similar solutions describing the evolution of a hot plasma in contact with a cold wall have been obtained in Ref. [24]. In these solutions, the thermoelectric effects were not taken into account. More recently, Velikovich, Giuliani and Zalesak [89] performed a detailed analysis of the evolution of a semi-infinite plasma slab in contact with a cold wall in a magnetized liner inertial fusion (MagLIF) regime, paying special attention to the thermal energy and magnetic flux losses in the plasma slab. This work became of great interest for the community since the authors retained the thermoelectric Nernst term in their formulation, which is suspected to degrade the magnetic flux conservation in a MagLIF implosion [84]. A particular feature of this analysis is that the cold wall, which aims to represent the liner, was treated as a solid wall with infinite heat capacity. The liner kept its solid integrity and did not ablate, and the hot plasma lost its thermal energy as a consequence of heat flux transferred to the cold liner.

However, in analytical models of the hot spot dynamics of spherical ICF implosions $[75,18,13,73,74,33]$, the shell surrounding the hot fuel is not treated as a solid 
wall, but rather as a cold dense plasma that ablates and increases the hot spot mass. It is argued that the heat losses are recycled back into the hot spot via the ablated shell material. The hot spot behaves as an adiabatic system, contrary to the assumptions made in the model of Velikovich and collaborators [89]. Since the inner side of the liner attains relatively high temperatures in MagLIF implosions, it is expected to ablate for the same reasons that lead to shell ablation in classic ICF implosions.

In the article presented in this chapter, the model analyzed in Ref. [89] is revisited taking into account liner mass ablation into the hot plasma. Consequently, the liner is not modeled as a solid cold wall but rather as a cold dense unmagnetized plasma made of the same material as the fuel (deuterium for simplicity). This choice is motivated by the high-gain MagLIF schemes [85], where a layer of cryogenic fuel is added on the inner side of the liner.

\subsection{Statement of the problem}

The evolution of a hot magnetized plasma occupying the semi-infinite space $x>0$, initially at rest and in contact with a cold dense unmagnetized plasma that extends towards $x<0$ is considered. Both plasmas are fully ionized, made of deuterium and in mechanical equilibrium. Plasma is modeled by a bi-fluid hydrodynamic description for electrons and ions, and the Braginskii [20] expressions for transport coefficients are used. It is assumed that the temperature and magnetic field in the cold plasma are zero and density is infinite; while $T_{0}, B_{0}$ refer to the initial temperature and magnetic field in the hot plasma.

The qualitative description of the motion goes as follows. Due to thermal conduction, the cold liner ablates into the hot plasma, compressing and cooling it down. The interface between the hot spot and the liner represents therefore an ablation front. Since the liner density is assumed to be infinite, the ablation front does not penetrate much into it, and is assumed to be fixed at $x=0$. Another surface of interest is the ablated border, $x_{b}(t)$, which separates the initial hot plasma placed at $x>0$ from the ablated liner material. The initial hot plasma loses its thermal energy by heat conduction through the ablated border, which is invested into heating up the ablated material. The cold dense plasma, on the contrary, stays at rest and unmagnetized since its thermal conductivity $\chi \sim T^{5 / 2}$ is low and the magnetic diffusivity $\nu_{m} \sim T^{-3 / 2}$ is high (it can be considered as a magnetic isolant): neither heat flux nor electrical currents can take place in it.

The magnetic field is subjected to convection and diffusion. Since the hot spot is very conductive, magnetic diffusion is typically negligible and its effect is confined within thin layers. In this context, convection dominates the magnetic field evolution. Two main mechanisms are responsible for it: plasma motion and the Nernst effect. Plasma motion, governed by the ablation process, convects the magnetic field from 
the liner towards the hot spot. The Nernst term, on its part, convects the magnetic field in the heat flux direction, i. e., from the hot spot towards the liner. Both effects are therefore opposite, and the resulting convection direction depends on the magnetization level of the plasma.

Magnetization of the plasma is characterized by the electron Hall parameter $x_{e}$, which is given by the product of the electron cyclotron frequency and the electron collision time, $x_{e}=\omega_{e} \tau_{e}$. Following Braginskii [20], the thermal conduction, magnetic diffusion and Nernst coefficients strongly depend on $x_{e}$, as shown in Fig. 2 in the article. Consequently, the plasma motion and the magnetic field evolution will be affected by the magnetization level.

\subsubsection{Methodology}

Plasma motion and magnetic field evolution in the hot spot are governed by the ion continuity, total momentum and total energy conservation equations, together with Faraday's law and the equation of state, Eqs. (1) - (5) in the article. The electric field is given by the electron momentum equation neglecting electron inertia, which stands for a generalized Ohm's law, whereas the electric current is given by Ampère's law, Eqs. (6) and (7) in the article, respectively. Due to the geometry of the problem, the motion is one-dimensional and the only independent variables are the streamwise direction $x$ and time $t$. Besides, ion velocity only presents a streamwise component and the magnetic field stays perpendicular to the motion. Under these conditions, the governing equations reduce to Eqs. (8) - (11) in the article. In the total energy equation, Eq. (10), the thermoelectric Nernst and Ettingshausen effects together with Joule dissipation appear as heat fluxes additionally to thermal conduction.

The system can be further simplified noticing that, in MagLIF implosions, the Mach number is typically small and the thermal-to-magnetic pressure ratio $\beta$ is high. Bearing this in mind, the momentum equation reduces to isobaricity: thermal pressure is constant and set by the initial conditions. In the energy equation, magnetic energy, thermoelectric and Joule effects, which are of order $O(1 / \beta)$, can be neglected. Thermal conduction becomes thereby the main mechanism governing plasma motion, and the energy equation can be integrated once yielding an explicit expression for velocity as a function of temperature and magnetic field, Eq. (13) in the article. Using these relations, the resulting governing equations can be reduced to ion continuity and induction equations: two equations for two unknowns, temperature and magnetic field.

Since the initial value problem lacks a characteristic length and velocity, it admits a self-similar solution in the variable $x / \sqrt{t}$. This kind of self-similar solution corresponds to a diffusive wave [93], which in this problem is governed by thermal conduction. Consequently, the self-similar coordinate $\eta=x / \sqrt{\kappa_{0} t}$ is introduced, with $\kappa_{0}$ being the thermal diffusivity. Temperature and magnetic field are normalized with their initial value in the hot plasma, and the velocity is consistently scaled 
with $\sqrt{\kappa_{0} / t}$. The ion continuity and induction equations normalized this way correspond to Eqs. (19) and (20) in the article. They form a system of two highly coupled ordinary differential equations of fourth order, and they need to be solved with the boundary conditions (21) and (22) for normalized temperature $\theta$ and magnetic field $\phi$. They impose zero temperature and magnetic field at the ablation front, $\eta=0$, and the recovery of the initial plasma state far from the liner, $\eta \rightarrow \infty$.

The system only depends on two free parameters: the magnetic Lewis number $\mathrm{Le}_{\mathrm{m}}$ and the electron Hall parameter of the unperturbed plasma $x_{e 0}$. The former stands for the ratio between the thermal and magnetic diffusivities of the unperturbed plasma, $\mathrm{Le}_{\mathrm{m}}=\kappa_{0} / \nu_{m 0}$, the latter being inversely proportional to the plasma electrical conductivity. This number is simply referred to as Le in the article. However, in order to be consistent with the nomenclature in Chapter 5, where Le stands for the usual Lewis number, it will be named $\mathrm{Le}_{\mathrm{m}}$ throughout all this document. It is typically large in MagLIF implosions, it ranges from $\operatorname{Le}_{\mathrm{m}} \sim 100$ at the beginning of the implosion to $\mathrm{Le}_{\mathrm{m}} \sim 10^{6}$ at the end, which indicates that magnetic diffusion is negligible in the length scale of the thermal wave. The electron Hall parameter can be of order unity, $x_{e 0} \sim 1$, at the beginning of the implosion, and it is expected to reach high values $x_{e 0} \sim 200$ at the end. It appears in conduction, Nernst and magnetic diffusion coefficients, since the electron Hall parameter anywhere in the hot spot can be related to the one in the unperturbed plasma as $x_{e}=x_{e 0} \phi \theta^{5 / 2}$. It is precisely due to the dependence of thermal conduction on magnetization that the ion continuity and induction equations are coupled. The magnetic Lewis number $\mathrm{Le}_{\mathrm{m}}$, electron Hall parameter $x_{e 0}$ and thermal-to-magnetic pressure ratio $\beta$ are related as $\mathrm{Le}_{\mathrm{m}} \sim \beta x_{e 0}^{2}$, shown in Eq. (15) in the article. Consequently, in order to be consistent with the large $\beta$ limit, only the case $\mathrm{Le}_{\mathrm{m}} \gg x_{e 0}^{2}$ has been investigated here. Arbitrary values of $\beta$ have been investigated in Chapter 4.

Apart from the temperature and magnetic field profiles in the hot spot, integral quantities such as mass ablation and magnetic flux losses per unit area in the hot spot, $m$ and $\Phi$, respectively, and thermal energy and magnetic flux losses per unit area in the initial hot plasma, $\mathcal{E}$ and $\tilde{\Phi}$, have been obtained. Interestingly, the thermal energy losses are proportional to the self-similar coordinate of the ablated border $\eta_{b}=x_{b} / \sqrt{\kappa_{0} t}$, as shown in Eq. (40). The latter is obtained from the fluid surface condition $V\left(\eta_{b}\right)=\eta_{b}$, which can be calculated a posteriori.

Mathematically, the problem consists of a boundary value problem of fourth order, with boundary conditions $\theta=\phi=0$ at $\eta=0$, and $\theta=\phi=1$ at $\eta \rightarrow \infty$. It has been solved numerically designing a shooting method. An asymptotic analysis of the governing equations for $\eta \ll 1$ has been performed to elucidate the shape of temperature and magnetic field profiles close to the ablation front. Two solutions have been identified. The first one gives $\left.\theta\right|_{\eta \ll 1} \sim \eta^{2 / 7},\left.\phi\right|_{\eta \ll 1} \sim \eta^{10 / 7}$. This solution yields a finite heat flux at the interface with the liner, $\eta=0$, and zero mass ablation rate. Physically, it corresponds to the solution obtained by Velikovich and collaborators [89], where the liner was modeled as a solid wall with infinite heat capacity. A second solution yielding finite mass ablation rate and zero heat flux at 
the liner corresponds to $\left.\theta\right|_{\eta \ll 1}=s_{\theta} \eta^{2 / 5},\left.\phi\right|_{\eta \ll 1}=\operatorname{Le}_{\mathrm{m}} s_{\phi} \eta^{8 / 5}$. This solution is the one explored in this paper, since it is expected to be more realistic to MagLIF physics. The parameters $s_{\theta}\left(\mathrm{Le}_{\mathrm{m}}, x_{e 0}\right), s_{\phi}\left(\mathrm{Le}_{\mathrm{m}}, x_{e 0}\right)$ are positive and obtained by solving the complete problem with the boundary conditions far from the liner. More specifically, the equations have been integrated, with an arbitrary choice for $s_{\theta}$ and $s_{\phi}$, starting at the ablation front and assuming the aforementioned profiles for temperature and magnetic field. The integration has been stopped at a large enough $\eta=\eta^{*}$, and the parameters $s_{\theta}$ and $s_{\phi}$ have been obtained by imposing $\theta\left(\eta^{*}\right)=1, \phi\left(\eta^{*}\right)=1$. It has been found that $\eta^{*}=8$ is sufficiently large to yield accurate results.

Apart from the numerical computation of the equations, analytic solutions have also been obtained in the case of low magnetization values, with and without Nernst term, and their derivation are detailed in Sec. III. A simplified model to study the highly magnetized limit analytically is proposed in the Appendix in the article. Singular perturbation theory has been applied to obtain the structure of the magnetic diffusive layers taking place, described in the next section.

\subsection{Results}

The unmagnetized limit $x_{e 0} \ll 1$ is firstly treated. In this case, continuity equation is uncoupled from induction, and the temperature profile is independent of the magnetic Lewis number. The liner ablates into the hot spot, compressing and cooling down the fuel. The ablated border is placed at $\eta_{b}=0.35$.

The magnetic field profile is obtained from the induction equation, and the solutions including the Nernst term and switching it off artificially have been compared. The relevant case in MagLIF of a large $\mathrm{Le}_{\mathrm{m}}$ has been given more attention. In this limit, and when the Nernst term is not taken into account, the magnetic field evolves frozen into the plasma. The ablated liner material carries layers with zero magnetic field, whereas the magnetic field in the initial hot plasma is amplified due to compression, presenting a peak at the right side of the ablated border, Fig. 4(a) in the article. It is at this surface where a thin layer takes place connecting both regions. In this layer, the magnetic field gradient is strong and diffusion becomes important. Asymptotic analysis performed for large $\mathrm{Le}_{\mathrm{m}}$ reveals that the width of this layer as well as the magnetic flux losses in the initial hot plasma scale as $O\left(1 / \sqrt{\mathrm{Le}_{\mathrm{m}}}\right)$. In the absence of the Nernst term, magnetic flux conservation is improved by increasing the hot plasma conductivity, i. e., increasing $\mathrm{Le}_{\mathrm{m}}$.

The Nernst term convects the magnetic field towards the liner, opposite to convection due to plasma motion. For magnetization values below $x_{e}=x_{e \text { th }}=4.37$, the former dominates. The magnetic field in the initial hot plasma is therefore convected into the ablated material by the Nernst term and pushed against the liner, Fig. 4(b). Here, it presents a peak that scales as $O\left(\mathrm{Le}_{\mathrm{m}}^{1 / 5}\right)$ and dissipates into a thin layer whose width scales as $O\left(1 / \sqrt{\mathrm{Le}_{\mathrm{m}}}\right)$. The Nernst term acts therefore as a powerful 
mechanism that enhances magnetic field diffusion close to the liner. Asymptotic analysis reveals that the magnetic flux losses both in the hot spot and in the initial hot plasma become independent of the magnetic Lewis number in a large $\mathrm{Le}_{\mathrm{m}}$ limit: $\Phi=0.40 B_{0} \sqrt{\kappa_{0} t}$ and $\tilde{\Phi}=0.045 B_{0} \sqrt{\kappa_{0} t}$, respectively. When the Nernst term is taken into account, increasing the hot plasma conductivity once the magnetic Lewis number is already large no longer improves the magnetic flux conservation.

Magnetizing the hot spot reduces the energy losses and improves the magnetic flux conservation. In a large magnetization $x_{e 0}$ limit, thermal conductivity is reduced by a factor of $x_{e 0}^{-2}$, yet the thermal energy losses are not suppressed as effectively. The presence of a cold unmagnetized liner makes the energy losses scale as $\mathcal{E} \sim x_{e 0}^{-7 / 10}$. When the hot spot is magnetized, the ablated plasma penetrates more slowly, and the hot plasma layers are closer to the liner, as shown in Fig. 5(a) in the article. The region where the temperature drops, named thermal layer with $\epsilon_{T}$ denoting its width, becomes thinner. The ablated border steps back too, and the last two quantities scale as $\epsilon_{T} \sim \eta_{b} \sim x_{e 0}^{-7 / 10}$. Two different regions in the hot spot can therefore be identified: one very close to the liner, $\eta \lesssim \epsilon_{T}$, where the plasma remains cold and unmagnetized, and one far from the liner, $\eta>\epsilon_{T}$, composed of hot and magnetized plasma. The Nernst convection velocity, proportional to the heat flux, is highly suppressed in the magnetized region, and magnetic field convection due to plasma motion dominates. The magnetic field moves therefore frozen into the plasma in this region. It presents a peak at the right side of the ablated border, where the ablated material compresses the initial hot plasma. The Nernst term is confined within the thermal layer, and it convects the magnetic field towards the liner, which presents a second bump there, Fig. 5(b) in the paper. The height of this bump is reduced with magnetization. A boundary layer follows this peak where the magnetic field is diffused, with $\epsilon_{B}$ denoting its width. This layer gets thicker with magnetization, and it scales as $\epsilon_{B} \sim x_{e 0}^{3 / 10} / \mathrm{Le}_{\mathrm{m}}^{1 / 2}$. It is seen that $\epsilon_{B} / \epsilon_{T} \sim x_{e 0} / \mathrm{Le}_{\mathrm{m}}^{1 / 2} \sim \beta^{-1 / 2}$. Therefore, in the large $\beta$ limit and highly magnetized case, magnetic diffusion is also confined within layers which are thin compared to the thermal wave width.

Mass ablation and magnetic flux losses in the hot spot and in the initial hot plasma are found to decrease with magnetization as $m \sim x_{e 0}^{-3 / 10}, \Phi \sim x_{e 0}^{-7 / 10}, \tilde{\Phi} \sim x_{e 0}^{-7 / 10}$, as shown in Fig. 6 in the paper. It is therefore concluded that the Nernst term enhances the magnetic flux losses by convecting the magnetic field towards the liner. It is a very effective process in the sense that the losses become independent of the hot spot conductivity. This effect is nevertheless reduced by magnetizing the hot plasma, as the Nernst term gets confined in the cold unmagnetized region of the hot spot adjacent to the liner.

Finally, the problem treated in Ref. [89] is compared to the one analyzed in this chapter. In the former, the liner does not ablate, and the motion is inverted: layers of plasma are convected from the hot spot towards the liner to increase density and restore isobaricity. The Nernst term reinforces the magnetic field convection due to plasma motion rather than opposing to it as in the problem analyzed in this chapter. As a consequence, the scaling laws for thermal and magnetic flux losses 
are different: $\mathcal{E} \sim x_{e 0}^{-1 / 2}, \Phi \sim x_{e 0}^{-1 / 2}$. It can be therefore concluded that taking into account mass ablation enhances the magnetic insulation effects in the plasma-liner interface evolution. 



\title{
Mass ablation and magnetic flux losses through a magnetized plasma-liner wall interface
}

\author{
F. García-Rubio ${ }^{\text {a) }}$ and J. Sanz \\ E.T.S.I. Aeronáutica y del Espacio, Universidad Politécnica de Madrid, Madrid 28040, Spain
}

(Received 4 May 2017; accepted 19 June 2017; published online 12 July 2017)

The understanding of energy and magnetic flux losses in a magnetized plasma medium confined by a cold wall is of great interest in the success of magnetized liner inertial fusion (MagLIF). In a MagLIF scheme, the fuel is magnetized and subsonically compressed by a cylindrical liner. Magnetic flux conservation is degraded by the presence of gradient-driven transport processes such as thermoelectric effects (Nernst) and magnetic field diffusion. In previous publications [Velikovich et al., Phys. Plasmas 22, 042702 (2015)], the evolution of a hot magnetized plasma in contact with a cold solid wall (liner) was studied using the classical collisional Braginskii's plasma transport equations in one dimension. The Nernst term degraded the magnetic flux conservation, while both thermal energy and magnetic flux losses were reduced with the electron Hall parameter $\omega_{e} \tau_{e}$ with a power-law asymptotic scaling $\left(\omega_{e} \tau_{e}\right)^{-1 / 2}$. In the analysis made in the present paper, we consider a similar situation, but with the liner being treated differently. Instead of a cold solid wall acting as a heat sink, we model the liner as a cold dense plasma with low thermal conduction (that could represent the cryogenic fuel layer added on the inner surface of the liner in a high-gain MagLIF configuration). Mass ablation comes into play, which adds notably differences to the previous analysis. The direction of the plasma motion is inverted, but the Nernst term still convects the magnetic field towards the liner. Magnetization suppresses the Nernst velocity and improves the magnetic flux conservation. Thermal energy in the hot plasma is lost in heating the ablated material. When the electron Hall parameter is large, mass ablation scales as $\left(\omega_{e} \tau_{e}\right)^{-3 / 10}$, while both the energy and magnetic flux losses are reduced with a power-law asymptotic scaling $\left(\omega_{e} \tau_{e}\right)^{-7 / 10}$. Published by AIP Publishing. [http://dx.doi.org/10.1063/1.4991391]

\section{INTRODUCTION}

Inertial confinement fusion (ICF) concepts rely on compressing a deuterium-tritium (DT) capsule by means of a high energy laser or an ion beam in order to attain ignition conditions. ${ }^{1}$ Implosion velocities of the order of $300 \mathrm{~km} / \mathrm{s}$ are required so that compression heating overcomes conduction and radiation losses, and fusion reactions can be triggered.

The presence of a magnetic field reduces the plasma thermal conductivity ${ }^{2}$ and enhances $\alpha$-particle energy deposition. ${ }^{3}$ Lower implosion velocities and higher yield experiments can therefore be conceived by magnetizing the fuel. Lindemuth and Kirkpatrick ${ }^{4,5}$ explored a low implosion velocity regime $(\sim 10 \mathrm{~km} / \mathrm{s})$ referred to as magnetized target fusion (MTF). More recently, Slutz and collaborators ${ }^{6}$ proposed a scenario in which a magnetized cylindrical capsule enclosed by a metallic liner (wall) is compressed at $100 \mathrm{~km} / \mathrm{s}$ by a pulsed-power machine. The attractiveness of this regime, denoted as magnetized liner inertial fusion (MagLIF), resides in the possibility of designing high gain configurations by adding a cryogenic DT layer on the inner surface of the liner. ${ }^{7}$

An important feature of magnetized implosions is the long time that the hot plasma and the cold liner stay in contact. An understanding of the heat and magnetic flux loss mechanisms through the plasma-liner interface is of great

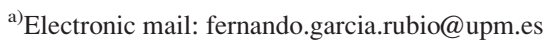

importance for the successful design of the implosions. Magnetized plasma wall confinement analysis dates back to the work of Alfvén and Smårs. ${ }^{8}$ Later on, $\mathrm{Chu}^{9}$ obtained self-similar solutions describing the evolution of a hot plasma in contact with a cold wall without thermoelectric effects, whereas Felber et al. ${ }^{10}$ derived self-similar solutions for a subsonic magnetized plasma compression.

In MagLIF implosions, the fuel inside the liner is preheated to $100-500 \mathrm{eV}$ by a laser. The Mach number Ma is less than unity (subsonic) at all stages of the implosion, and the motion can be considered isobaric. In a recent publication, Velikovich and collaborators ${ }^{11}$ studied magnetic flux and thermal energy losses in a semi-infinite plasma slab in contact with a wall in a MagLIF context. Special attention was paid on how the Nernst effect, which accounts for magnetic field convection in the heat flux direction, degraded the magnetic flux conservation even when the plasma is very conductive. A particular feature of this analysis is that the cold liner was treated as a solid wall with infinite heat capacity. Heat was transferred from the plasma into the liner, resulting in thermal energy losses. The plasma was set into motion in order to restore isobaricity, convecting layers of hot plasma towards the liner.

In previous publications concerning the hot spot dynamics of ICF capsules, ${ }^{12-14}$ the imploding shell enclosing the plasma is considered to be a high density cold gas with low thermal conductivity. Contrary to the analysis performed in Ref. 11, the implosion is adiabatic (no heat flux towards the 
shell) and the cold material is ablated from the shell into the hot spot, pushing the plasma towards the center of the capsule.

The purpose of the present paper is to study energy losses and magnetic flux conservation in a high gain MagLIF configuration, ${ }^{7}$ which is similar to ICF hot spot dynamics and differs from Ref. 11 in the fact that the hot plasma is adiabatically compressed by a cold DT layer with low thermal conductivity, and mass is ablated into the hot spot. In order to elucidate how magnetization reduces energy and magnetic flux losses, the implosion dynamics has been disregarded and only plasma motion due to transport terms (field gradients) is considered. We study therefore the evolution of a hot magnetized plasma (hot spot) in contact with a cold unmagnetized plasma (liner) in planar geometry, as represented in the artistic scheme in Fig. 1.

This paper is organized as follows. In Sec. II, the problem is described and the governing equations are discussed. They can be reduced to a system of two partial differential equations for temperature and magnetic field in which the solution presents a self-similar structure. In Sec. III, the system is solved in the case of low magnetization, in which the two equations get uncoupled. In Sec. IV, the effect of magnetization on magnetic flux losses and mass ablation is thoroughly studied. Finally, in Sec. V, conclusions are drawn.

\section{GOVERNING EQUATIONS}

We consider at $t=0$ a hot plasma medium at rest with a uniform temperature $T_{0}$, density $n_{0}$, and thermal pressure $p_{0}$, occupying the semi-infinite space $x>0$. It is initially magnetized with a uniform magnetic field $\vec{B}_{0}=B_{0} \vec{e}_{z}$. The hot plasma is in contact at $x=0$ with a cold denser unmagnetized plasma $\left(T \ll T_{0}, B=0\right)$ that extends towards $x<0$. Both plasmas are made of deuterium $(Z=1, A=2)$. They are fully ionized and in mechanical equilibrium. The hot plasma would crudely represent the hot spot, while the cold plasma stands for the liner. Quasineutrality, same ion and electron temperatures and ideal gas hypotheses are assumed.

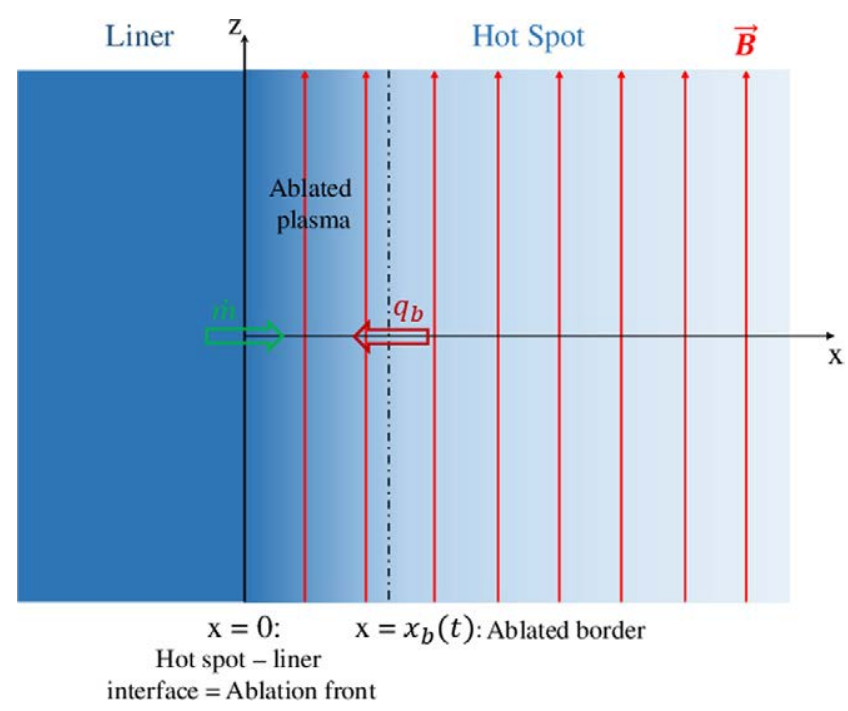

FIG. 1. Artistic scheme of the problem presented. The color gradation represents ion density. Arrows in red stand for magnetic field lines.
The hot plasma is cooled down and set into motion due to thermal conduction, while the magnetic field is subjected to convection and diffusion. The cold dense plasma, on the contrary, stays at rest and unmagnetized since its thermal conductivity $\chi \sim T^{5 / 2}$ is low and the magnetic diffusivity $D_{m} \sim T^{-3 / 2}$ is high (it can be considered as a magnetic isolant); neither heat flux nor electrical currents can take place in it.

The hot plasma ion density $n$, thermal pressure $p=p_{e}$ $+p_{i}$, temperature $T$, ion velocity $\vec{v}$, and magnetic field $\vec{B}$ evolution is governed by the ion continuity, total momentum, and total energy equations neglecting electron inertia and plasma viscosity, together with Faraday's law and the equation of state

$$
\begin{gathered}
\frac{\partial n}{\partial t}+\nabla \cdot(n \vec{v})=0 \\
m_{i} n \frac{\partial \vec{v}}{\partial t}+m_{i} n \vec{v} \cdot \nabla \vec{v}=-\nabla p+\frac{1}{c} \vec{j} \times \vec{B} \\
\frac{\partial}{\partial t}\left(\frac{1}{2} m_{i} n v^{2}+\frac{1}{\gamma-1} p+\frac{B^{2}}{8 \pi}\right) \\
+\nabla \cdot\left[\left(\frac{1}{2} m_{i} n v^{2}+\frac{\gamma}{\gamma-1} p\right) \vec{v}+\frac{c}{4 \pi} \vec{E} \times \vec{B}\right]=-\nabla \cdot \vec{q} \\
\frac{\partial \vec{B}}{\partial t}=-c \nabla \times \vec{E} \\
p=2 n T
\end{gathered}
$$

where $m_{i}$ refers to the ion mass and $\gamma=5 / 3$ is the adiabatic index. The electric field $\vec{E}$ can be obtained from the electron momentum equation, whereas the electric current $\vec{j}$ is given by Ampère's law

$$
\begin{gathered}
\vec{E}=-\frac{1}{c} \vec{v} \times \vec{B}+\frac{1}{e c n} \vec{j} \times \vec{B}-\frac{1}{e n}\left(\frac{\nabla p}{2}-\vec{R}\right), \\
\nabla \times \vec{B}=\frac{4 \pi}{c} \vec{j} .
\end{gathered}
$$

We use Braginskii's ${ }^{15}$ expressions and notations for the transport coefficients to relate the electron and ion heat fluxes $\vec{q}=\vec{q}_{e}+\vec{q}_{i}$ and the change of momentum between ions and electrons $\vec{R}$ to the plasma variables.

The system of equations (1)-(5) has to be completed with appropriate boundary conditions. We impose that far from the liner, $x \rightarrow \infty$, the plasma recovers the initial state $p=p_{0}, T=T_{0}$, and $\vec{B}=\vec{B}_{0}$. At the interface with the liner, $x=0$, we require that the plasma temperature be equal to the cold plasma temperature, $T \ll T_{0}$, and the velocity and magnetic field be null, $\vec{v}=0, \vec{B}=0$.

Due to the geometry of the problem, the only independent variables are the streamwise direction $x$ and time $t$. Besides, ion velocity only presents a streamwise component $\vec{v}=v(x, t) \vec{e}_{x}$, the magnetic field stays perpendicular to the motion $\vec{B}=B(x, t) \vec{e}_{z}$, the currents flow in the spanwise direction $\vec{j}=j(x, t) \vec{e}_{y}$, and the electric field has two components $\vec{E}=E_{x}(x, t) \vec{e}_{x}+E_{y}(x, t) \vec{e}_{y}$. Equations (1)-(4) become 


$$
\begin{gathered}
\frac{\partial n}{\partial t}+\frac{\partial}{\partial x}(n v)=0 \\
m_{i} n \frac{\partial v}{\partial t}+m_{i} n v \frac{\partial v}{\partial x}=-\frac{\partial}{\partial x}\left(p+\frac{B^{2}}{8 \pi}\right) \\
\frac{\partial}{\partial t}\left(\frac{1}{2} m_{i} n v^{2}+\frac{1}{\gamma-1} p+\frac{B^{2}}{8 \pi}\right)+\frac{\partial}{\partial x}\left[\left(\frac{1}{2} m_{i} n v^{2}+\frac{\gamma}{\gamma-1} p+\frac{B^{2}}{4 \pi}\right) v\right] \\
=\frac{\partial}{\partial x}[\underbrace{\chi_{\perp} \frac{\partial T}{\partial x}}_{\text {Cond. }}+\frac{c \beta_{\curlywedge}^{u T}}{4 \pi e n}(\underbrace{B \frac{\partial T}{\partial x}}_{\text {Nernst }}+\underbrace{T \frac{\partial B}{\partial x}}_{\text {Ettingsh. }})+\underbrace{\frac{D_{m \perp}}{4 \pi} B \frac{\partial B}{\partial x}}_{\text {Joule }}] \\
\frac{\partial B}{\partial t}+\frac{\partial}{\partial x}(v B)=\frac{\partial}{\partial x}(\underbrace{D_{m \perp} \frac{\partial B}{\partial x}}_{\text {Joule }}+\underbrace{\frac{c \beta_{\Lambda}^{u T}}{e n B} \frac{\partial T}{\partial x} B}_{\text {Nernst }})
\end{gathered}
$$

The coefficient $\chi_{\perp}$ stands for electron plus ion conductivities, $\beta_{\wedge}^{u T}$ refers to the transport coefficient for the thermoelectric Nernst and Ettingshausen effects, and $D_{m \perp}=\alpha_{\perp} c^{2} / 4 \pi e^{2} n^{2}$ is the magnetic diffusion coefficient appearing in the Joule effect.

Denoting $\beta=8 \pi p_{0} / B_{0}^{2}$ the characteristic thermal to magnetic pressure ratio, the analysis of the system (8)-(11) is performed in the double limit small Mach number $\mathrm{Ma} \ll 1$ and $\beta \gg 1$, typical in a MagLIF regime. ${ }^{11}$ With this simplification, both Eqs. (9) and (10) can be integrated once, yielding

$$
\begin{gathered}
p=p_{0}, \\
v=\frac{\gamma-1}{\gamma p_{0}} \chi_{\perp} \frac{\partial T}{\partial x}+v_{\infty} .
\end{gathered}
$$

Thermal pressure is therefore uniform (isobaricity), and equal to its value far from the liner. Magnetic energy, thermoelectric and Joule effects, of order $1 / \beta$, are neglected in Eq. (10) and plasma is set into motion as a result of thermal conduction. The constant of integration $v_{\infty}$ is obtained by applying the boundary condition $\left.v\right|_{x=0}=0$, and it represents the plasma velocity far from the liner, where the initial plasma state is recovered. Two situations can be considered at this stage depending on how the liner is modeled. Due to thermal conduction, the hot plasma close to the liner is cooled down and mass is advected there in order to increase the density and restore isobaricity. This mass has either to come from far away from the liner $(x \rightarrow \infty)$ or to be ablated at the hot spot-liner interface $(x=0)$. Besides, energy conservation balance established in the hot plasma states that the heat flux $q$ at the liner is equal to enthalpy convection far from the liner $\gamma p_{0} v_{\infty} /(\gamma-1)$. Therefore, if the liner is treated as a solid wall, as done in Ref. 11, mass cannot be ablated at the liner, then the plasma velocity far from the liner has to be negative and finite $\left(v_{\infty}<0\right)$ in order to restore isobaricity, and consequently heat flux $q$ is finite and negative at the liner due to energy balance. However, if the liner is treated as a high density cold plasma with low thermal conductivity, any heat flux coming from the hot plasma would not propagate inside. An overpressure would thereby be originated at the contact interface $x=0$ that would instantaneously ablate the cold material into the hot plasma, reducing the heat flux and restoring isobaricity. Consequently, the heat flux at the liner is assumed to be zero, which by energy balance sets $v_{\infty}=0$. The plasma velocity is then proportional to minus the heat flux: $v=-(\gamma-1) q / \gamma p_{0}$. This second model, in which the hot plasma-liner interface $(x=0)$ represents an ablation front, is taken in this paper since we consider that it is more realistic in a high gain MagLIF configuration.

\section{A. Normalization and self-similarity}

The plasma density, temperature, and magnetic field are scaled with their initial value in the hot plasma: $\rho=n / n_{0}$, $\theta=T / T_{0}$, and $\phi=B / B_{0}$. Density is related to temperature through the equation of state $\rho=1 / \theta$. The thermal conductivity coefficient can thereby be written as $\chi_{\perp}=\bar{K} T_{0}^{5 / 2} \theta^{5 / 2}$ $\mathcal{P}_{c}\left(x_{e}\right)$, where $\bar{K}=n \tau_{e} \gamma_{0} / T^{3 / 2} m_{e}$ is a conductivity constant, and $\tau_{e}, m_{e}$ are the electron collision time and mass. The thermoelectric transport coefficient is proportional to the conductivity coefficient $c \beta_{\wedge}^{u T} / e n B=\left[(\gamma-1) \chi_{\perp} / \gamma p\right] \mathcal{P}_{n}\left(x_{e}\right)$, and consequently also vanishes at the liner. The magnetic diffusion coefficient is expressed as $D_{m \perp}=\bar{D} T_{0}^{-3 / 2} \theta^{-3 / 2} \mathcal{P}_{d}\left(x_{e}\right)$, with $\bar{D}=c^{2} m_{e} T^{3 / 2} \alpha_{0} / 4 \pi e^{2} n \tau_{e}$ being a diffusivity constant. The terms $\mathcal{P}_{c}, \mathcal{P}_{n}$, and $\mathcal{P}_{d}$ account for the effect of magnetization on the transport terms, Fig. 2. They are rational functions of the electron Hall parameter (electron cyclotron frequency times the electron collision time) $x_{e}=\omega_{e} \tau_{e}$ $=\left(e B / m_{e} c\right) \tau_{e}$

$$
\begin{aligned}
& \mathcal{P}_{c}\left(x_{e}\right)=\frac{\gamma_{1}^{\prime} x_{e}^{2}+\gamma_{0}^{\prime}}{\gamma_{0} \Delta_{e}}+\sqrt{\frac{2 m_{e}}{m_{i}}} \frac{2 x_{i}^{2}+2.645}{\gamma_{0} \Delta_{i}}, \\
& \mathcal{P}_{n}\left(x_{e}\right)=\frac{2 \gamma}{\gamma-1} \frac{\beta_{1}^{\prime \prime} x_{e}^{2}+\beta_{0}^{\prime \prime}}{\gamma_{1}^{\prime} x_{e}^{2}+\gamma_{0}^{\prime}+\sqrt{\frac{2 m_{e}}{m_{i}}} \frac{\Delta_{e}}{\Delta_{i}}\left(2 x_{i}^{2}+2.645\right)}, \\
& \mathcal{P}_{d}\left(x_{e}\right)=\frac{1-\frac{\alpha_{1}^{\prime} x_{e}^{2}+\alpha_{0}^{\prime}}{\Delta_{e}}}{\alpha_{0}},
\end{aligned}
$$

with $\Delta_{e}=x_{e}^{4}+\delta_{1} x_{e}^{2}+\delta_{0}$ and $\Delta_{i}=x_{i}^{4}+2.70 x_{i}^{2}+0.677$, and $x_{i}=\omega_{i} \tau_{i}=\sqrt{2 m_{e} / m_{i}} x_{e}$ standing for the ion magnetization. The electron Hall parameter anywhere in the hot plasma can be written as a function of its value far from the liner $x_{e 0}$ and the dimensionless temperature and magnetic fields

$$
x_{e}=x_{e 0} \phi \theta^{5 / 2} .
$$

In an unmagnetized plasma, thermal conduction is mainly due to electrons because of their small mass. However, they get magnetized for lower magnetic field intensities compared to the ions, and when electron conduction is suppressed, the ions carry the heat transport. For large magnetization values $\left(x_{e}>\sqrt{m_{i} / 2 m_{e}} \approx 43\right)$, the ions also get magnetized and the thermal conductivity decreases asymptotically as $P_{c} \approx 28 / x_{e}^{2}$.

We define the magnetic Lewis number Le as the ratio between thermal and magnetic diffusivities in an unmagnetized plasma, $\kappa_{0}=(\gamma-1) \bar{K} T_{0}^{7 / 2} / \gamma p_{0}$ and $\nu_{m 0}=\bar{D} T_{0}^{-3 / 2}$, 

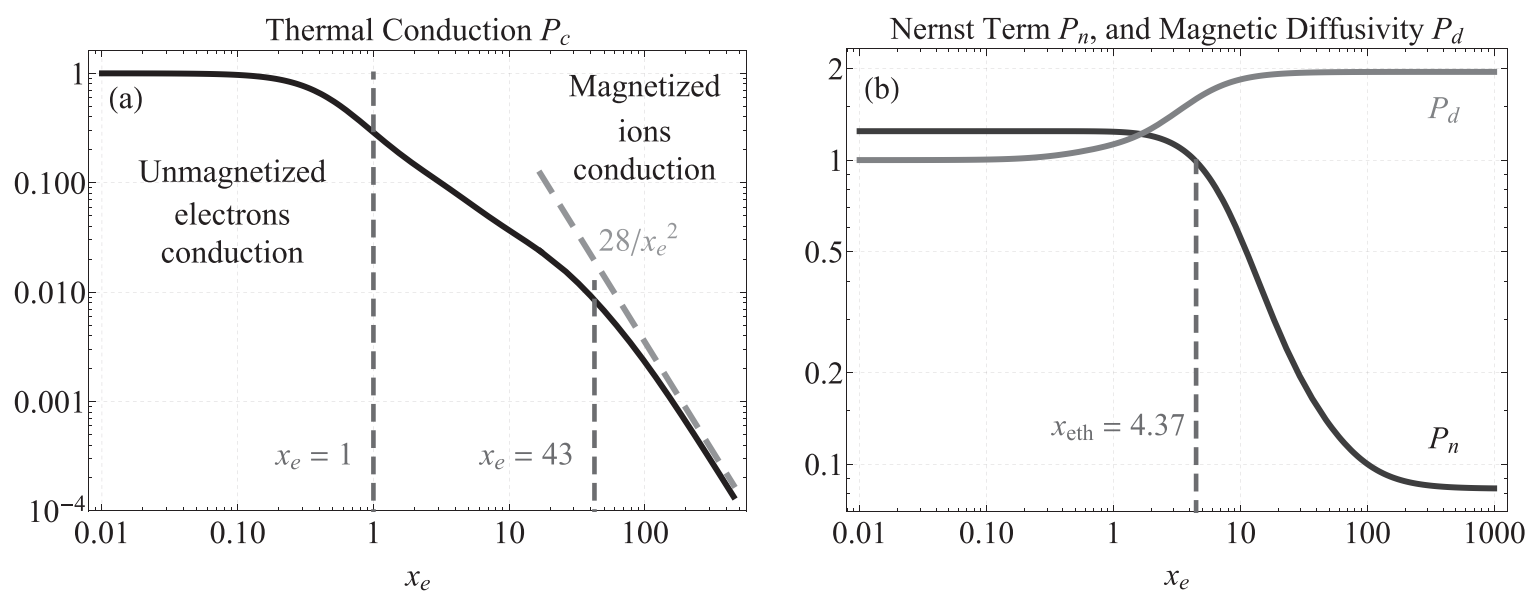

FIG. 2. Transport coefficient dependence on magnetization.

respectively. It is related to the pressure ratio $\beta$ and electron Hall parameter $x_{e 0}$ through

$$
\text { Le }=\frac{\kappa_{0}}{\nu_{m 0}}=\frac{\gamma-1}{8 \gamma} \frac{\gamma_{0}}{\alpha_{0}} \beta x_{e 0}^{2}=0.31 \beta x_{e 0}^{2} .
$$

Since the initial value problem proposed lacks a characteristic length and velocity, the solution to Eqs. (8)-(11) is sought under the form of a self-similar diffusive wave. ${ }^{16}$ We introduce, consequently, the independent self-similar variable

$$
\eta=\frac{x}{\sqrt{\kappa_{0} t}}(\eta \geq 0)
$$

Consistently, the plasma ion velocity is scaled self-similarly

$$
V(\eta)=2 \frac{v(x, t)}{\sqrt{\kappa_{0} / t}}=2 \mathcal{P}_{c} \theta^{5 / 2} \frac{\mathrm{d} \theta}{\mathrm{d} \eta} .
$$

The ion continuity equation (8) is rewritten as

$$
(V-\eta) \frac{\mathrm{d} \rho}{\mathrm{d} \eta}+\rho \frac{\mathrm{d} V}{\mathrm{~d} \eta}=0
$$

which, by the use of the equation of state and the self-similar velocity (17) reads

$$
\eta \frac{\mathrm{d} \theta}{\mathrm{d} \eta}+2 \theta^{2} \frac{\mathrm{d}}{\mathrm{d} \eta}\left(\mathcal{P}_{c} \theta^{3 / 2} \frac{\mathrm{d} \theta}{\mathrm{d} \eta}\right)=0
$$

The induction equation (11) likewise normalized gives

$$
\begin{aligned}
& -\eta \frac{\mathrm{d} \phi}{\mathrm{d} \eta}+\frac{\mathrm{d}}{\mathrm{d} \eta}\left[2 \mathcal{P}_{c}\left(1-\mathcal{P}_{n}\right) \theta^{5 / 2} \frac{\mathrm{d} \theta}{\mathrm{d} \eta} \phi\right] \\
& =\frac{2}{\mathrm{Le}} \frac{\mathrm{d}}{\mathrm{d} \eta}\left(\frac{\mathcal{P}_{d}}{\theta^{3 / 2}} \frac{\mathrm{d} \phi}{\mathrm{d} \eta}\right)
\end{aligned}
$$

Two opposite effects take part in the convection term in Eq. (20): magnetic field convection by the plasma motion, in the opposite direction to the conduction heat flux, and convection due to the Nernst term, in the same direction. For $x_{e}<x_{e t h}(=4.37), 1-P_{n}$ is negative and the latter prevails, see Fig. 2(b).
Continuity and induction equations (19) and (20) form a system of two ordinary differential equations of fourth order for the normalized temperature and magnetic field profiles $\theta(\eta), \phi(\eta)$. They are coupled through the dependence of the thermal conductivity on the magnetization and need to be completed with the normalized boundary conditions

$$
\begin{gathered}
\theta(0)=0, \quad \theta(\eta \rightarrow \infty)=1, \\
\phi(0)=0, \quad \phi(\eta \rightarrow \infty)=1 .
\end{gathered}
$$

This system only depends on two free parameters: the magnetic Lewis number Le and the electron Hall parameter of the unperturbed plasma $x_{e 0}$. In a typical MagLIF implosion, ${ }^{6,11}$ the Lewis number evolves from Le $\sim 100$ at the beginning of the implosion to Le $\sim 10^{6}$ at the end, whereas the electron Hall parameter goes from $x_{e 0} \sim 1$ to $x_{e 0} \sim 200$.

\section{B. Mass ablation and magnetic flux losses}

We define the mass increase $m$ and magnetic flux losses $\Phi$ per unit area in the hot spot as

$$
\begin{aligned}
& m=\int_{0}^{\infty}\left(n-n_{0}\right) \mathrm{d} x, \\
& \Phi=\int_{0}^{\infty}\left(B_{0}-B\right) \mathrm{d} x .
\end{aligned}
$$

Integrating in space Eqs. (8) and (11), the time derivative of $m$ and $\Phi$ can be related to the mass ablation rate and magnetic diffusion at the liner $(x=0)$, reading

$$
\frac{\mathrm{d} m}{\mathrm{~d} t}=\left.n v\right|_{x=0}, \quad \frac{\mathrm{d} \Phi}{\mathrm{d} t}=\left.D_{m \perp} \frac{\partial B}{\partial x}\right|_{x=0} .
$$

The mass ablation rate and magnetic diffusion, for their part, depend on the shape of the temperature and magnetic field profiles close to the liner, where the plasma is cold and unmagnetized. An asymptotic analysis of the Eqs. (19) and (20) performed for $\eta \ll 1$ reveals that the temperature and magnetic field profiles close to the liner take the form

$$
\left.\theta\right|_{\eta \ll 1}=s_{\theta} \eta^{2 / 5},\left.\quad \phi\right|_{\eta \ll 1}=s_{\phi} \operatorname{Le} \eta^{8 / 5},
$$


where the parameters $s_{\theta}\left(\mathrm{Le}, x_{e 0}\right)$ and $s_{\phi}\left(\mathrm{Le}, x_{e 0}\right)$ are positive and obtained by solving the complete problem with the boundary conditions far from the liner. The plasma density and velocity profiles close to the liner read

$$
\left.\rho\right|_{\eta \ll 1}=\frac{1}{s_{\theta} \eta^{2 / 5}},\left.\quad V\right|_{\eta \ll 1}=s_{V} \eta^{2 / 5}=\frac{4}{5} s_{\theta}^{7 / 2} \eta^{2 / 5} .
$$

These expansions yield a finite mass ablation rate since $\left.\rho V\right|_{\eta \ll 1}=s_{V} / s_{\theta}$. The similar problem solved with a solid liner in Ref. 11 gives the asymptotic expansions $\theta \sim \eta^{2 / 7}$, $\rho \sim \eta^{-2 / 7}$ and $V \sim \eta$ negative, resulting in zero mass ablation: $\rho V \sim \eta^{5 / 7} \rightarrow 0$.

The time derivative of the mass increase and magnetic flux losses can consequently be expressed in terms of the parameters $s_{\theta}, s_{\phi}$

$$
\begin{aligned}
\frac{\mathrm{d} m}{\mathrm{~d} t} & =\frac{2 n_{0}}{5} \sqrt{\frac{\kappa_{0}}{t}} s_{\theta}^{5 / 2}, \\
\frac{\mathrm{d} \Phi}{\mathrm{d} t} & =\frac{8 B_{0}}{5} \sqrt{\frac{\kappa_{0}}{t}} \frac{s_{\phi}}{s_{\theta}^{3 / 2}} .
\end{aligned}
$$

Since the parameters $s_{\theta}, s_{\phi}$ do not depend on time, these equations can be integrated yielding

$$
\begin{gathered}
m=\frac{4 n_{0}}{5} \sqrt{\kappa_{0} t} s_{\theta}^{5 / 2}, \\
\Phi=\frac{16 B_{0}}{5} \sqrt{\kappa_{0} t} \frac{s_{\phi}}{s_{\theta}^{3 / 2}} .
\end{gathered}
$$

\section{Ablated border}

We define the ablated border as the surface separating the ablated material from the hot plasma initially occupying the space $x>0$. It is by definition a fluid surface since the fluid particles move along with it. Letting $x_{b}(t)$ denote its position (Fig. 1), it is computed by stating that the hot spot mass increase $m$ is equal to the ablated mass

$$
m=\int_{0}^{x_{b}} n \mathrm{~d} x .
$$

The hot spot (region $x>0$ ) is therefore formed by the initial hot plasma, situated downstream of the ablated border $\left(x>x_{b}\right)$, and the ablated material, placed between the liner and the ablated border $\left(0<x<x_{b}\right)$. Naming $\eta_{b}(t)=x_{b}(t) /$ $\sqrt{\kappa_{0} t}$ and using Eq. (18), the next relation is derived

$$
\int_{0}^{x_{b}} n \mathrm{~d} x=n\left(x_{b}, t\right) \sqrt{\kappa_{0} t}\left[\eta_{b}-V\left(\eta_{b}\right)\right]+m .
$$

Applying Eq. (32) to this relation leads the ablated border self-similar coordinate $\eta_{b}$ to satisfy

$$
\eta_{b}=V\left(\eta_{b}\right)
$$

This equation always has a solution because the velocity profile is greater than $\eta$ close to the liner and vanishes far from it. The ablated border travels then at constant $\eta_{b}>0$, which depends on the magnetic Lewis number and magnetization intensity: $\eta_{b}\left(\mathrm{Le}, x_{e 0}\right)$, and the fluid surface condition $\mathrm{d} x_{b} / \mathrm{d} t=$ $v\left(x_{b}, t\right)$ is well satisfied. As a consequence of relation (34), the value of $\eta_{b}$ also represents the minus dimensionless heat flux at the ablated border $q_{b}$. Thermal energy from the hot plasma is this way drained to heat up the cold ablated material at a rate

$$
q_{b}=-\frac{\gamma}{2(\gamma-1)} p_{0} \sqrt{\frac{\kappa_{0}}{t}} \eta_{b} .
$$

From the ion continuity (18), it can be inferred that $V^{\prime}=$ 0 and $V^{\prime \prime}=\rho^{\prime} / \rho$ at $\eta=\eta_{b}$, with the prime denoting derivative with respect to $\eta$. Hence, if the density profile is monotonous (which is the case in all the computations shown later on), the velocity and consequently the heat flux attain a maximum there. The plasma velocity relative to the ablated border is then negative everywhere, so the initial hot plasma is seen as being convected towards it. The ablated border could therefore be an equivalent to the plasma-solid wall interface considered in Ref. 11, with the exception that the temperature is finite at this surface in the model proposed in the present paper.

Two other quantities of interest are the thermal energy losses $\mathcal{E}$ and magnetic flux losses $\tilde{\Phi}$ per unit area in the initial hot plasma, defined as

$$
\begin{gathered}
\mathcal{E}=\int_{0}^{\infty} \frac{p_{0}}{\gamma-1} \mathrm{~d} x-\int_{x_{b}}^{\infty} \frac{p_{0}}{\gamma-1} \mathrm{~d} x, \\
\tilde{\Phi}=\int_{0}^{\infty} B_{0} \mathrm{~d} x-\int_{x_{b}}^{\infty} B \mathrm{~d} x .
\end{gathered}
$$

The time derivatives of $\mathcal{E}$ and $\tilde{\Phi}$ are related to the heat flux and magnetic field diffusion and convection by Nernst at the ablated border, respectively

$$
\begin{gathered}
\frac{\mathrm{d} \mathcal{E}}{\mathrm{d} t}=-\frac{q_{b}}{\gamma}, \\
\frac{\mathrm{d} \tilde{\Phi}}{\mathrm{d} t}=\left.\left(D_{m \perp} \frac{\partial B}{\partial x}+\frac{c \beta_{\wedge}^{u T}}{e n B} \frac{\partial T}{\partial x} B\right)\right|_{x=x_{b}} .
\end{gathered}
$$

These equations can be integrated yielding

$$
\begin{gathered}
\mathcal{E}=\frac{p_{0}}{\gamma-1} \sqrt{\kappa_{0} t} \eta_{b}, \\
\tilde{\Phi}=2 B_{0} \sqrt{\kappa_{0} t} s_{b},
\end{gathered}
$$

with $s_{b}=s_{b}\left(\right.$ Le, $\left.x_{e 0}\right)$ given by

$$
s_{b}=\left.\left(\frac{\mathcal{P}_{d}}{\operatorname{Le} \theta^{3 / 2}} \frac{\mathrm{d} \phi}{\mathrm{d} \eta}+\mathcal{P}_{n} \mathcal{P}_{c} \theta^{5 / 2} \frac{\mathrm{d} \theta}{\mathrm{d} \eta} \phi\right)\right|_{\eta=\eta_{b}} .
$$

The thermal energy losses of the initial hot plasma previously defined would be the equivalent to the thermal energy losses referred to in Ref. 11.

\section{SOLUTION FOR UNMAGNETIZED PLASMA}

In this section, the system (19) and (20) is analyzed in a low magnetized limit $x_{e 0} \ll 1$. The transport polynomials 
become $\mathcal{P}_{c}=\mathcal{P}_{d}=1$, whereas $\mathcal{P}_{n}=1.24$. The velocity expression simplifies to $V=2 \theta^{5 / 2} \mathrm{~d} \theta / \mathrm{d} \eta$.

\section{A. Temperature and velocity}

Ion continuity (19) is uncoupled from the induction equation, reading

$$
\eta \frac{\mathrm{d} \theta}{\mathrm{d} \eta}+2 \theta^{2} \frac{\mathrm{d}}{\mathrm{d} \eta}\left(\theta^{3 / 2} \frac{\mathrm{d} \theta}{\mathrm{d} \eta}\right)=0
$$

This equation, together with the boundary conditions (21), allows us to obtain the temperature profile and, consequently, the velocity profile. They are independent of the magnetic Lewis number and depicted in Fig. 3. The value of $s_{\theta}$ yields $s_{\theta}\left(\right.$ Le, $\left.x_{e 0}=0\right)=1.045$, whereas the ablated border takes place at $\eta_{b}=0.35$.

\section{B. Magnetic field}

The induction equation (20) in the unmagnetized limit simplifies to

$$
-\eta \frac{\mathrm{d} \phi}{\mathrm{d} \eta}+2 \delta \frac{\mathrm{d}}{\mathrm{d} \eta}\left(\theta^{5 / 2} \frac{\mathrm{d} \theta}{\mathrm{d} \eta} \phi\right)=\frac{2}{\operatorname{Le}} \frac{\mathrm{d}}{\mathrm{d} \eta}\left(\frac{1}{\theta^{3 / 2}} \frac{\mathrm{d} \phi}{\mathrm{d} \eta}\right),
$$

where the parameter $\delta$ has been introduced to study the effect of Nernst convection. If this term is taken into account, then $\delta=1-\mathcal{P}_{n}(0)=-0.24$ and the magnetic field is convected towards the liner, whereas if the Nernst convection is artificially switched off, then $\delta=1$ and the magnetic field is exclusively convected by the plasma motion towards the hot spot. The induction equation (44) has to be solved with the boundary conditions (22).

\section{Nernst effect off}

Numerical solutions of Eq. (44) when the Nernst term is not taken into account $(\delta=1)$ are depicted in Fig. 4(a) for different magnetic Lewis numbers. When the Lewis number is moderate, magnetic field diffusion is important in all the

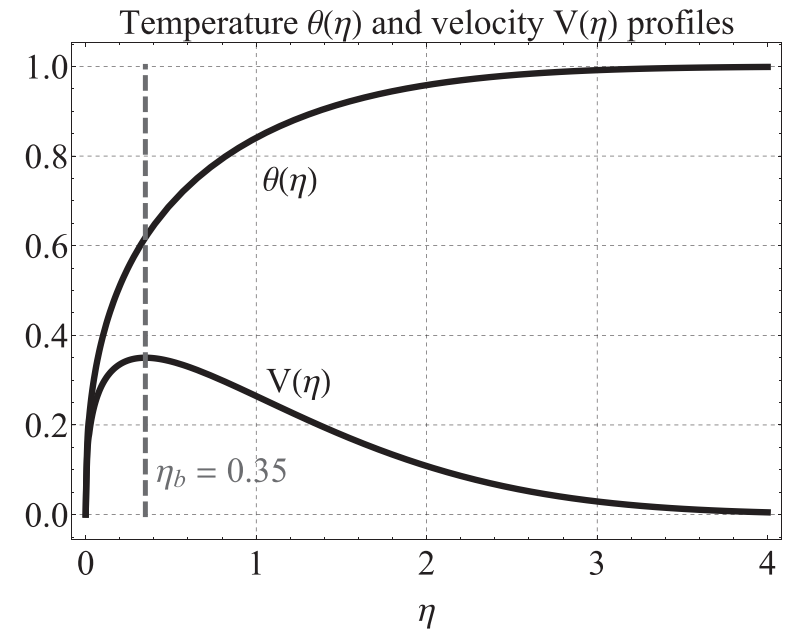

FIG. 3. Temperature $\theta(\eta)$ and velocity $V(\eta)$ self-similar profiles for unmagnetized plasma $x_{e 0}=0$. Position of the ablated border $\eta_{b}=0.35$.
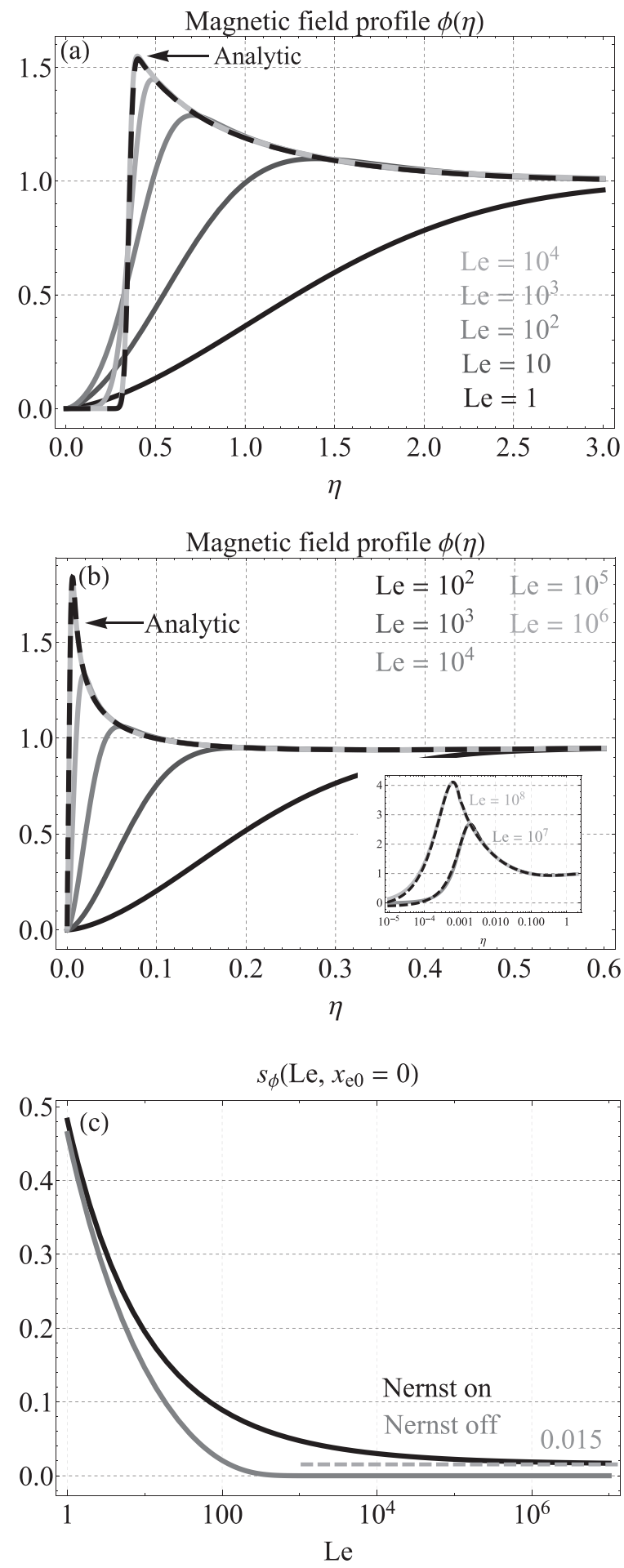

FIG. 4. Magnetic field for unmagnetized plasma $x_{e 0}=0$. (a) Self-similar magnetic field profile $\phi(\eta)$ without the Nernst term. Analytical solution for Le $=10^{4}$ in dashed lines. (b) Self-similar magnetic field profile $\phi(\eta)$ with the Nernst term. Analytical solutions for $\mathrm{Le}=10^{6}, 10^{7}$ and $10^{8}$ in dashed lines. (c) Parameter $s_{\phi}$.

domain. However, when the Lewis number is large, the magnetic field evolution is mainly governed by the convection due to plasma motion, with diffusion being confined to thin layers. The cold and unmagnetized plasma ablated at the liner pushes and compresses the initial hot plasma and the magnetic field therein. 
Equation (44) can be solved analytically in a large Le limit Le $\gg 1$. To the leading order, diffusion can be neglected and the magnetic field moves along with the fluid ("frozen field"). Letting $\phi_{o}$ denote the leading order solution (outer solution), it reads

$$
\phi_{o}(\eta)=C \rho(\eta)
$$

with $C$ a constant of integration. The magnetic field frozen in the ablated plasma penetrating into the hot spot is null, so the solution before the ablated border is $\phi_{o}^{-}=0$, which gives $s_{\phi}=0$. The magnetic field profile frozen in the initial hot plasma, downstream of the ablated border, yields $\phi_{o}^{+}=\rho$. Both branches have to be matched at $\eta=\eta_{b}$, where $\phi_{o}^{-}=0$ and $\phi_{o}^{+}=\phi_{b}=1.62$. Therefore, strong gradients take place at the ablated border and diffusion becomes important.

In order to obtain the structure of this layer, the selfsimilar coordinate is expanded as $\eta=\eta_{b}+\epsilon_{B} \xi$, while the magnetic field inside the layer (inner solution) is written as $\phi=\phi_{i}(\xi)=\phi_{i 0}(\xi)+\epsilon_{B} \phi_{i 1}(\xi)+O\left(\epsilon_{B}^{2}\right)$, with $\epsilon_{B} \ll 1$ measuring the width of the layer and $\mathrm{d} \phi_{i} / \mathrm{d} \xi \sim O(1)$. Two terms need to be retained in the inner expansion $\phi_{i}$ in order to ensure a $\mathcal{C}^{1}$ matching with the outer solution $\phi_{o}$. Temperature, velocity, and external magnetic field close to the ablated border take the form $\theta=\theta_{b}+\theta_{b}^{\prime} \epsilon_{B} \xi+O\left(\epsilon_{B}^{2}\right), V=V_{b}+V_{b}^{\prime \prime} \epsilon_{B}^{2} \xi^{2} / 2+O\left(\epsilon_{B}^{3}\right)$, and $\phi_{e}^{+}=\phi_{b}+\phi_{b}^{\prime} \epsilon_{B} \xi+O\left(\epsilon_{B}^{2}\right)$, with $\theta_{b}=0.62, \theta_{b}^{\prime}=0.58$, $V_{b}=0.35, V_{b}^{\prime \prime}=-0.94$, and $\phi_{b}^{\prime}=-1.52$ being the value of temperature, velocity, and external magnetic field and their derivatives at the ablated border. Inserting these ansätze into Eq. (44) and requiring diffusion to be important gives the width of the diffusion layer

$$
\left.\epsilon_{B}\right|_{\text {Nernst Off }}=\sqrt{\frac{2}{\theta_{b}^{3 / 2} \mathrm{Le}}}=\frac{2.05}{\sqrt{\mathrm{Le}}} .
$$

The leading order of the expanded induction equation reads

$$
\frac{\mathrm{d}^{2} \phi_{i 0}}{\mathrm{~d} \xi^{2}}+\xi \frac{\mathrm{d} \phi_{i 0}}{\mathrm{~d} \xi}=0
$$

which has to be solved with the boundary conditions $\phi_{i 0}(\xi \rightarrow-\infty)=0, \phi_{i 0}(\xi \rightarrow \infty)=\phi_{b}$, yielding

$$
\phi_{i 0}(\xi)=\frac{\phi_{b}}{2}\left[1+\operatorname{Erf}\left(\frac{\xi}{\sqrt{2}}\right)\right]
$$

where Erf is the usual error function. The order $O\left(\epsilon_{B}\right)$ of the expanded induction equation reads

$$
\frac{\mathrm{d}^{2} \phi_{i 1}}{\mathrm{~d} \xi^{2}}+\xi \frac{\mathrm{d} \phi_{i 1}}{\mathrm{~d} \xi}=V_{b}^{\prime \prime} \xi\left(\phi_{i 0}+\frac{\xi}{2} \frac{\mathrm{d} \phi_{i 0}}{\mathrm{~d} \xi}\right)+\frac{3}{2} \frac{\theta_{b}^{\prime}}{\theta_{b}} \frac{\mathrm{d}}{\mathrm{d} \xi}\left(\xi \frac{\mathrm{d} \phi_{i 0}}{\mathrm{~d} \xi}\right),
$$

which has to be solved numerically with the boundary conditions $\phi_{i 1}(\xi \rightarrow-\infty)=0$ and $\phi_{i 1}(\xi \rightarrow-\infty)=\phi_{b}^{\prime} \xi$. The numerical and analytic profiles for large Le are compared in Fig. 4(a), showing good agreement.

The magnetic flux losses in the initial hot plasma are measured by the parameter $s_{b}$, which in this large Le limit and Nernst off yields

$$
s_{b}=\frac{1}{\sqrt{\operatorname{Le}}} \frac{\phi_{b}}{\theta_{b}^{3 / 4} \sqrt{4 \pi}}=\frac{0.65}{\sqrt{\mathrm{Le}}} .
$$

Therefore, the magnetic field in the initial hot plasma is compressed by the ablated border without losses for large Lewis numbers, with the peak of the compression being $\phi_{b}=1.62$. The magnetic flux in the whole hot spot is consequently conserved in this limit, with $s_{\phi} \rightarrow 0$ as seen in Fig. 4(c).

\section{Nernst effect on}

Numerical solutions of Eq. (44) taking into account the Nernst term $(\delta=-0.24)$ for different magnetic Lewis numbers are shown in Fig. 4(b). When the Lewis number is large, the magnetic field evolution is governed by convection in the heat flux direction. It traverses the ablated border and accumulates close to the liner, where it is dissipated in a thin layer in order to satisfy the boundary condition $\phi(0)=0$. In this thin layer, the magnetic field gradients are strong and diffusion becomes important.

In this large Le limit, the induction equation can be solved analytically. Letting $\phi_{o}$ denote the leading order solution (outer solution) of Eq. (44) in a large Lewis limit, it satisfies

$$
\frac{\mathrm{d} \phi_{o}}{\mathrm{~d} \eta}=-\delta \phi_{o} \frac{\mathrm{d} V / \mathrm{d} \eta}{\delta V-\eta} .
$$

The magnetic field attains a local minimum at the ablated border $\phi_{o}\left(\eta_{b}\right)=0.94$, while close to the liner it takes the form $\phi_{o}(\eta \ll 1)=s_{\phi_{o}} \eta^{-2 / 5}$, with $s_{\phi_{o}}=0.20$. In order to describe the structure of the boundary layer that takes place close to the liner, the self-similar coordinate is expanded as $\eta=\epsilon_{B} \xi$, while the magnetic field inside the layer (inner solution) is written as $\phi=\phi_{i}(\xi)+O\left(\epsilon_{B}\right)$, with $\mathrm{d} \phi_{i} / \mathrm{d} \xi \sim O(1)$. Requiring diffusion to be important gives the width of this boundary layer

$$
\left.\epsilon_{B}\right|_{\text {Nernst on }}=\sqrt{\frac{2}{s_{\theta}^{3 / 2} s_{V} \mathrm{Le}}} \approx \frac{1.42}{\sqrt{\mathrm{Le}}} .
$$

Inserting this expansion into the induction equation yields the governing equation for the inner solution

$$
\delta \frac{\mathrm{d}}{\mathrm{d} \xi}\left(\xi^{2 / 5} \phi_{i}\right)=\frac{\mathrm{d}}{\mathrm{d} \xi}\left(\frac{1}{\xi^{3 / 5}} \frac{\mathrm{d} \phi_{i}}{\mathrm{~d} \xi}\right)
$$

which has to satisfy the boundary condition at the liner $\phi_{i}(0)=0$, resulting in

$$
\phi_{i}(\xi)=Q\left[\int_{0}^{\xi} \hat{\xi}^{3 / 5} \exp \left(-\delta \hat{\xi}^{2} / 2\right) \mathrm{d} \hat{\xi}\right] \exp \left(\delta \xi^{2} / 2\right) .
$$

The inner solution attains a local maximum at $\xi=\xi_{\max }$ $=4.21$, taking the value $\phi_{i}\left(\xi_{\max }\right)=Q /\left(-\delta \xi_{\max }^{2 / 5}\right)$. The parameter $Q$ is obtained by matching the inner solution $\phi_{i}$ with the outer $\phi_{o}$, resulting in 


$$
Q=-\delta \frac{s_{\phi_{o}} s_{V}^{1 / 5} s_{\theta}^{3 / 10}}{2^{1 / 5}} \mathrm{Le}^{1 / 5}=0.042 \mathrm{Le}^{1 / 5}
$$

Consequently, the value of the magnetic field at the peak gives $\phi_{\max }=\phi_{i}\left(\xi_{\max }\right)=0.10 \mathrm{Le}^{1 / 5}$, while the magnetic field profile close to the liner takes the form

$$
\left.\phi\right|_{\eta \ll 1}=-\frac{5}{16} \delta s_{\phi_{o}} s_{\theta}^{3 / 2} s_{V} \operatorname{Le} \eta^{8 / 5}=0.015 \mathrm{Le} \eta^{8 / 5} .
$$

The parameter $s_{\phi}$ attains then $s_{\phi}=0.015$ for large Lewis numbers which, by virtue of Eq. (29), implies significant magnetic flux losses even for a perfectly conductive plasma (infinite Le). The value of $s_{\phi}$ with and without Nernst, shown in Fig. 4(c) for different Le, quantifies how the Nernst term enhances the degradation of the magnetic flux conservation. On the other hand, the parameter $s_{b}$ takes the value $s_{b}=\left.(1-\delta)\left(\theta^{5 / 2} \mathrm{~d} \theta / \mathrm{d} \eta \phi\right)\right|_{\eta=\eta_{b}}=0.20$ for large Le. In sum, the magnetic field is convected by the Nernst velocity from the initial hot plasma into the ablated material, resulting in magnetic flux losses for the initial hot plasma $\tilde{\Phi}=0.40 B_{0} \sqrt{\kappa_{0} t}$; it accumulates close the liner and dissipates in a thin layer, entailing magnetic flux losses for the whole hot spot $\Phi=0.045 B_{0} \sqrt{\kappa_{0} t}$.

\section{SOLUTION FOR MAGNETIZED PLASMA}

Magnetic insulation of the fuel is intended in order to reduce thermal energy losses. According to the effect of magnetization on thermal conduction, Fig. 2(a), a straightforward estimation would yield that the thermal energy losses $\mathcal{E}$ would scale as $x_{e 0}^{-2}$ when the magnetization $x_{e 0}$ is large. Besides, the Nernst velocity, proportional to the heat flux, would be suppressed to the same extent as the energy losses, improving the magnetic flux conservation. However, since the hot plasma is surrounded by a cold unmagnetized liner, which forces the magnetization to decrease drastically in the plasma close to it, these estimations result to be overrated and the energy and magnetic flux losses are not reduced as efficiently with magnetization.

Temperature and magnetic field profiles for different magnetization intensities and Le $=10^{5}$ are depicted in Fig. 5 .
Hot plasma layers are closer to the liner when the magnetization increases. We name the thermal layer to the region where the temperature drops, and $\epsilon_{T}$ refers to its width. It can be seen that both the thermal layer width and the parameter $s_{\theta}$ decrease with $x_{e 0}$. Plasma and Nernst velocities are thereby confined within this layer. If $x_{e 0}>x_{e t h}$, the plasma velocity overcomes the Nernst velocity at the outer part of it, while closer to the liner, where the magnetization is low, the Nernst velocity becomes important and convects the magnetic field towards the liner. The coordinate where the magnetic field convection direction is inverted is referred to as $\eta_{\mathrm{th}}$ : $x_{e}\left(\eta_{t h}\right)=x_{e t h}=4.37$. For $\eta>\eta_{\mathrm{th}}$, the magnetic field undergoes a compression pushed by the ablated border, presenting a first bump. A second bump takes place for $\eta<\eta_{\text {th }}$ due to the accumulation of the magnetic field pushed against the liner, which is dissipated in a diffusive layer close to it. The height of the second bump decreases with the magnetization, eventually disappearing for very large $x_{e 0}$, the reason for this being that the thermal layer width becomes comparable to the diffusive layer.

Two situations can thereby take place depending on Le and $x_{e 0}$ : the thermal layer being thicker than the diffusive layer $\left(\epsilon_{T} \gg \epsilon_{B}\right)$, and both layers being of the same order of magnitude $\left(\epsilon_{T} \sim \epsilon_{B}\right)$. In the first case, plasma demagnetization is due to the temperature drop, whereas in the second case, both magnetic field and temperature decrease are responsible for the demagnetization. In the Appendix, a simple model has been developed in the asymptotic case $\epsilon_{T} \gg \epsilon_{B}$, and the scaling laws for the thermal layer width, parameter $s_{\theta}$ and ablated border position $\eta_{b}$ derived in this limit yield

$$
\epsilon_{T} \sim \frac{1}{x_{e 0}^{7 / 10}}, \quad s_{\theta} \sim \frac{1}{x_{e 0}^{3 / 25}}, \quad \eta_{b} \sim \frac{1}{x_{e 0}^{7 / 10}} .
$$

The mass ablated and the thermal energy losses scale therefore as $m \sim x_{e 0}^{-3 / 10}$ and $\mathcal{E} \sim x_{e 0}^{-7 / 10}$. Besides, from direct observation at the computations, the magnetic flux losses are also found to scale as $\Phi \sim x_{e 0}^{-7 / 10}$. Magnetization reduces the energy losses by decreasing the thermal diffusivity, and improves the magnetic flux conservation by reducing the
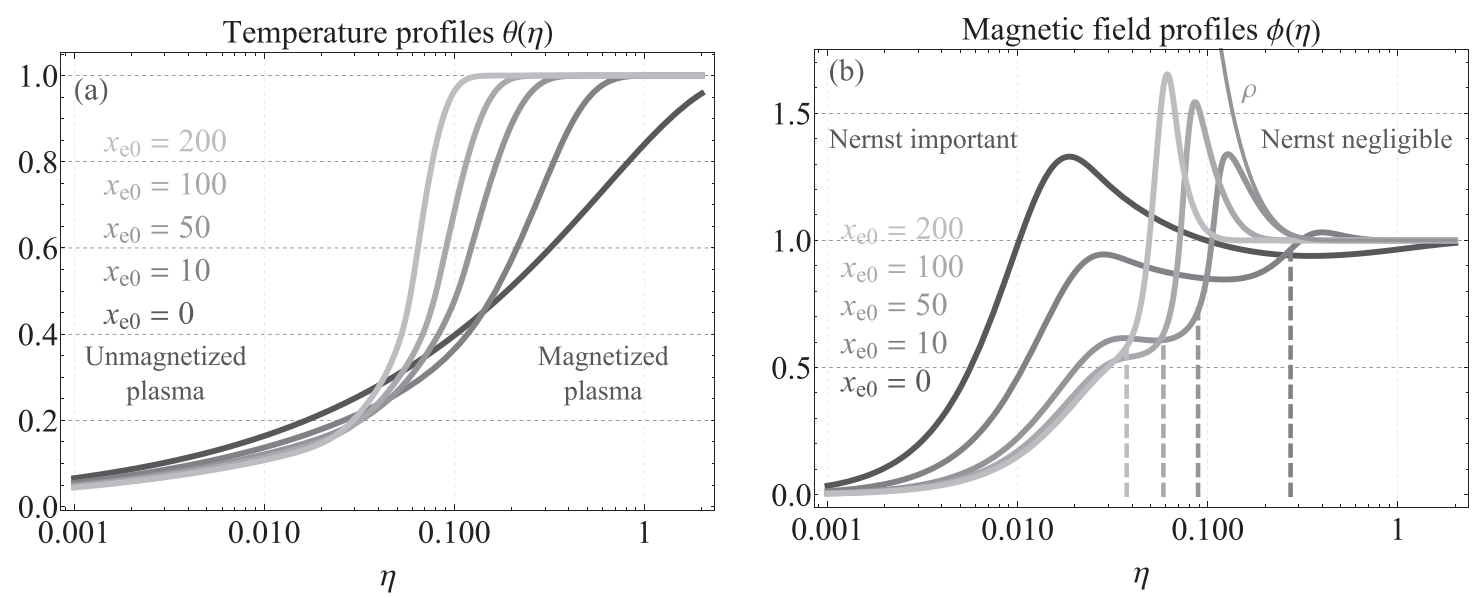

FIG. 5. Temperature $\theta(\eta)$ and magnetic field $\phi(\eta)$ self-similar profiles for Le $=10^{5}$ and $x_{e 0}=0,10,50$, and 200. The dashed lines refer to $\eta_{\text {th }}$, the position where $x_{e}=x_{e \text { th }}=4.37$. Density profile $\rho(\eta)$ in (b) is plotted for Le $=10^{5}$ and $x_{e 0}=50$. 
Nernst velocity and confining its effect to a thin layer of unmagnetized plasma close to the liner. However, the losses are reduced less than what the first estimations at the beginning of this section predict $\left(x_{e 0}^{-7 / 10}\right.$ compared to $\left.x_{e 0}^{-2}\right)$.

In order to check the validity of the assumption $\left(\epsilon_{T} \gg \epsilon_{B}\right)$, the width of the diffusive layer is estimated yielding

$$
\epsilon_{B} \sim \frac{x_{e 0}^{3 / 10}}{\mathrm{Le}^{1 / 2}} .
$$

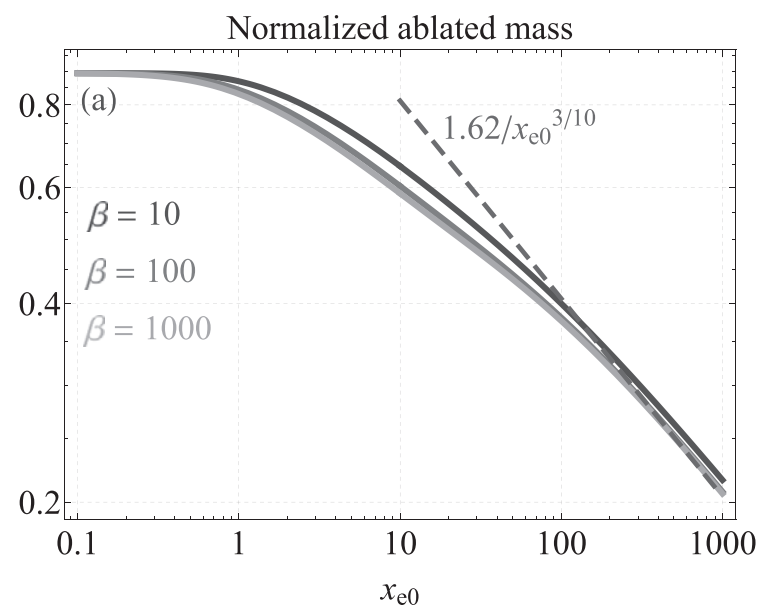

Normalized thermal energy losses

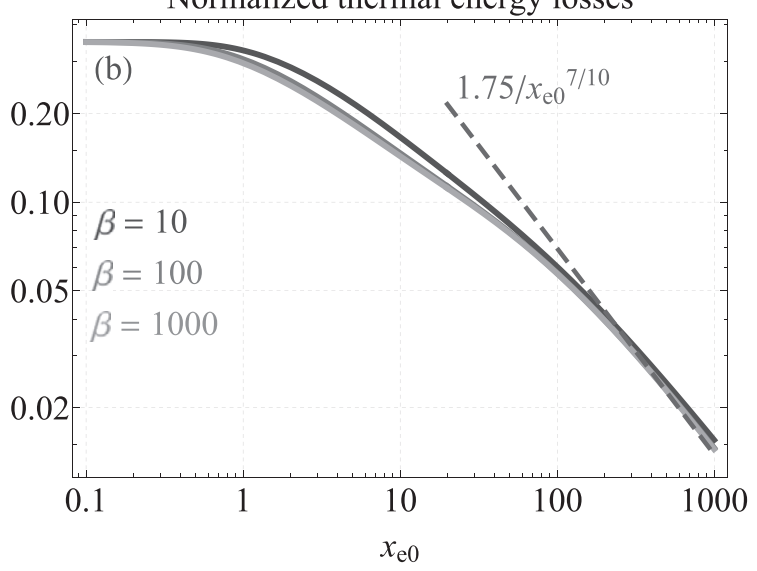

Normalized magnetic flux loss

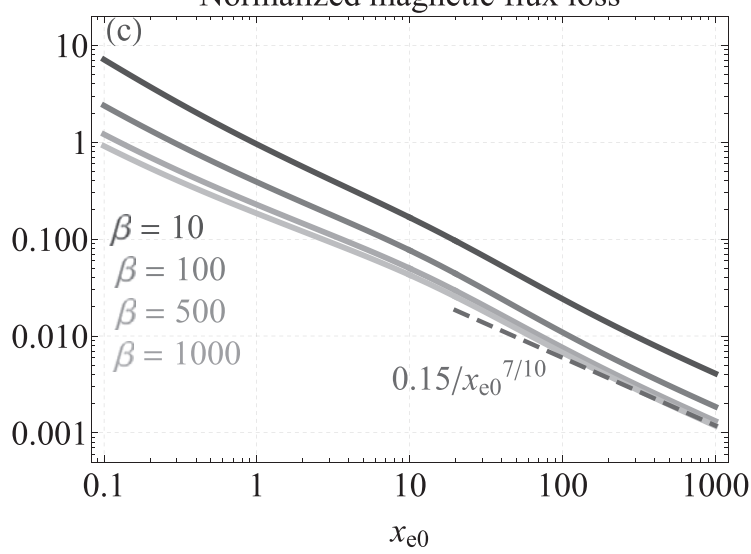

FIG. 6. Effect of the magnetization $x_{e 0}$ on (a) normalized ablated mass $m / n_{0}$ $\sqrt{\kappa_{0} t}=4 s_{\theta}^{5 / 2} / 5$, (b) normalized thermal energy losses $(\gamma-1) \mathcal{E} / p_{0} \sqrt{\kappa_{0} t}=\eta_{b}$ and (c) normalized magnetic flux losses $\Phi / B_{0} \sqrt{\kappa_{0} t}=16 s_{\phi} / 5 s_{\theta}^{3 / 2}$.

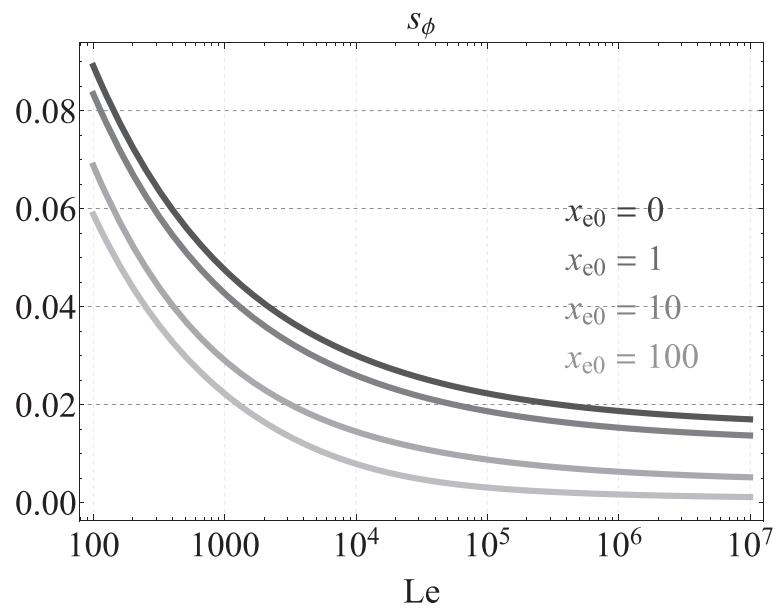

FIG. 7. Effect of the magnetic Lewis number on the parameter $s_{\phi}$.

The limit $\epsilon_{T} \gg \epsilon_{B}$ therefore breaks when Le $\sim x_{e 0}^{2}$, and the diffusive layer becomes as thick as the thermal layer. Interestingly, it is precisely for Le $\sim x_{e 0}^{2}$ when the finite $\beta$ effects become noticeable, see Eq. (15). Consequently, the situation $\epsilon_{T} \leq \epsilon_{B}$ is not contemplated in this analysis since it would not be consistent with the $\beta \gg 1$ limit previously assumed.

Computations of the effect of the magnetization $x_{e 0}$ on the ablated mass, energy, and magnetic flux losses are shown in Fig. 6. These variables are plotted for increasing $x_{e 0}$ and keeping a constant and large $\beta$ (same $\epsilon_{T} / \epsilon_{B}$ ratio). For large magnetization, $x_{e 0}>100$, the curves follow the trend predicted by the scaling laws derived. In Table I, these laws are compared to the results obtained by Velikovich and collaborators in Ref. 11, and it can be seen how mass ablation enhances the reduction of thermal energy and magnetic flux losses when the plasma is magnetized $\left(x_{e 0}^{-7 / 10}\right.$ compared to $\left.x_{e 0}^{-1 / 2}\right)$.

The effect of the Lewis number is analyzed in Fig. 7, where the parameter $s_{\phi}$ is depicted varying Le and different constant $x_{e 0}$. When the Lewis number increases, the diffusive layer becomes thinner and entirely takes place in the unmagnetized region close to the liner. The temperature profile gets independent of the Lewis number, and so does the parameter $s_{\phi}$. Therefore, as it also occurs in the unmagnetized case, the magnetic flux losses are mainly governed by the Nernst conduction and get independent of the Lewis number in a large Le limit.

TABLE I. Comparison between scaling laws derived in Ref. 11 and in this work. Thermal energy losses in Ref. $11, \mathcal{Q}$, refer to energy losses in the whole hot spot region $x>0$. Thermal energy losses in the present work, $\mathcal{E}$, correspond to energy losses in the initial hot plasma region $x>x_{b}$.

\begin{tabular}{lcccc}
\hline \hline & $\begin{array}{c}\text { Mass } \\
\text { ablation }\end{array}$ & $\begin{array}{c}\text { Heat flux at the } \\
\text { liner wall }\end{array}$ & $\begin{array}{c}\text { Thermal energy } \\
\text { losses }\end{array}$ & $\begin{array}{c}\text { Magnetic flux } \\
\text { losses }\end{array}$ \\
\hline Ref. 11 & $m=0$ & $\left.q\right|_{x=0} \sim \frac{1}{x_{e 0}^{1 / 2}}$ & $\mathcal{Q} \sim \frac{1}{x_{e 0}^{1 / 2}}$ & $\Phi \sim \frac{1}{x_{e 0}^{1 / 2}}$ \\
$\begin{array}{l}\text { Present } \\
\text { work }\end{array}$ & $m \sim \frac{1}{x_{e 0}^{3 / 10}}$ & $\left.q\right|_{x=0}=0$ & $\mathcal{E} \sim \frac{1}{x_{e 0}^{7 / 10}}$ & $\Phi \sim \frac{1}{x_{e 0}^{7 / 10}}$ \\
\hline
\end{tabular}




\section{CONCLUSIONS}

An analysis of the physical mechanisms that originate energy and magnetic flux losses in high-gain MagLIF implosions has been performed. The analysis focuses on the diffusive nature of the process by studying the evolution of a hot magnetized plasma in contact with a cold unmagnetized liner wall.

The problem is considered to be isobaric, with the plasma motion being governed by heat conduction. Material is ablated at the liner and penetrates into the hot spot, cooling and compressing the hot plasma. The interface between the ablated material and the hot plasma is named ablated border. Heat flux transports energy drained from the hot plasma to heat up the ablated material.

The magnetic field is subjected to convection and diffusion. Two different effects convect the magnetic field in opposite directions: plasma motion and the Nernst effect. The latter convects the magnetic field in the heat flux direction and prevails for low magnetization $\left(x_{e}<4.3\right)$. Magnetic diffusivity is measured by the magnetic Lewis number, which is usually large in a MagLIF context; hence, diffusion is confined to thin layers.

In a low magnetized limit, the Nernst term transports the magnetic field from the initial hot plasma to the ablated plasma. Once there, the magnetic field is pushed against the liner, and dissipated in a thin layer close to it. The Nernst term acts therefore as an engine that enhances the magnetic diffusion close to the liner, resulting in the degradation of the magnetic flux conservation even when the plasma is very conductive (large magnetic Lewis number).

Magnetizing the plasma reduces the energy losses and improves the magnetic flux conservation. In a large magnetization $x_{e 0}$ limit, thermal conductivity is reduced by a factor of $x_{e 0}^{-2}$, yet the thermal energy losses are not suppressed as effectively. The presence of a cold unmagnetized liner makes the energy and magnetic flux losses scale as $x_{e 0}^{-7 / 10}$. The Nernst velocity is reduced to the same extent as the thermal energy losses, resulting in an improvement of the magnetic flux conservation. When the plasma is magnetized, the ablated plasma penetrates more slowly, and the hot plasma layers are closer to the liner. The magnetic field profile presents two different regions. Far from the liner, where the plasma is magnetically insulated, the Nernst velocity is suppressed and the magnetic field is convected by the plasma motion towards the hot spot. Close to the liner, where the magnetization is low, the
Nernst velocity advects the magnetic field towards the liner, enhancing diffusion. Therefore, the effect of the Nernst velocity is reduced by confining it to a thin layer of unmagnetized plasma close to the liner.

Apart from the interest in unveiling the mechanisms that lead to the degradation of the magnetic flux in magnetized implosions, this work serves as a valuable source for a test case design and comparison to test the validity of MHD codes. Future work would imply taking into account finite $\beta$ effects and considering a liner made of a different material, in which case the ablated plasma would have different properties to the rest of the hot spot.

\section{ACKNOWLEDGMENTS}

This research was supported by the Spanish Ministerio de Economía y Competitividad, Project No. ENE2014-54960R, and by the Spanish Ministerio de Educación, Cultura y Deporte under the national research program FPU, Grant No. FPU 14/04879.

\section{APPENDIX: SIMPLE MODEL FOR MAGNETIZED HOT SPOT}

When the diffusive layer is thin compared to the thermal layer, $\epsilon_{B} \ll \epsilon_{T}$, plasma demagnetization is due to the temperature drop. In this limit, a simple model can be derived by neglecting the effect of the magnetic field on the electron Hall parameter $x_{e}$ since, in the length scale of the thermal problem, the magnetic field is of order unity almost everywhere: $x_{e}=x_{e 0} \theta^{5 / 2}$. The thermal problem gets this way uncoupled from the magnetic field in the $\epsilon_{B} \ll \epsilon_{T}$ limit. Performing the change of variables: $y=x_{e 0}^{7 / 10} \eta, z=x_{e 0} \theta^{5 / 2}$, ion continuity (19) transforms into

$$
y \frac{\mathrm{d} z}{\mathrm{~d} y}+2 z^{7 / 5} \frac{\mathrm{d}}{\mathrm{d} y}\left[\mathcal{P}_{c}(z) \frac{\mathrm{d} z}{\mathrm{~d} \eta}\right]=0 .
$$

This equation has to be completed with the boundary conditions $z(0)=0$ and $z(y \rightarrow \infty)=x_{e 0}$. The latter can be substituted by $\mathrm{d} z / \mathrm{d} y(0)=a$, with $a$ being an arbitrary constant. This forms a Cauchy problem that has a unique solution; therefore, a relation $a\left(x_{e 0}\right)$ can be obtained, which is plotted in Fig. 8(a). It tends to a limit value $a=a_{l}=1.837$ when $x_{e 0}$ is large. The profile $\theta(y)^{5 / 2}$ is depicted in Fig. 8(b) for increasing $a$ values. For $a$ close to $a_{l}$, i.e., large $x_{e 0}$, the
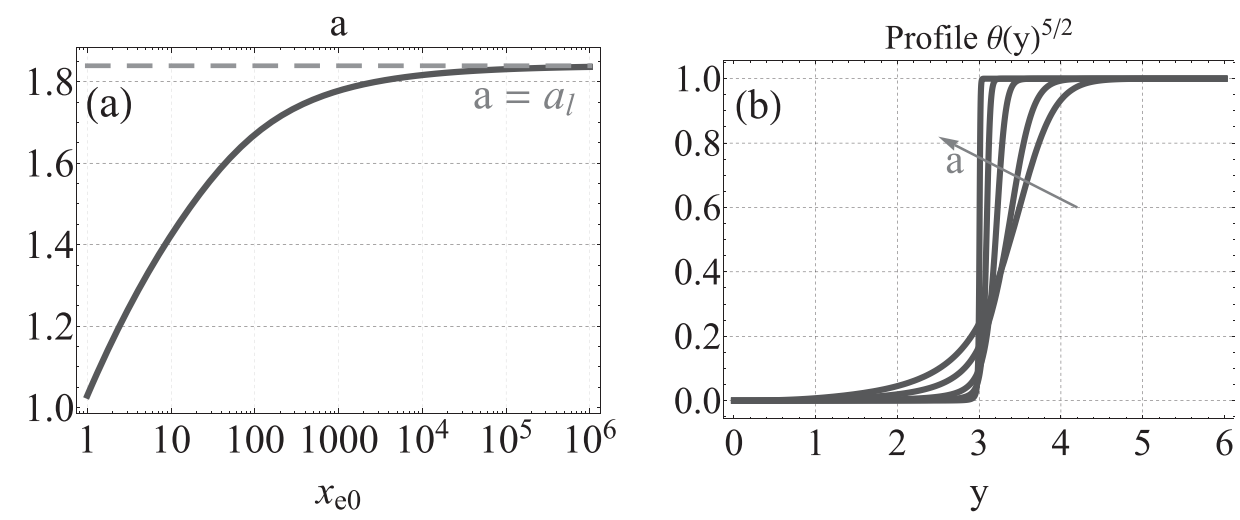

FIG. 8. Temperature slope $a$ and temperature profiles in the simple model developed in the Appendix. 
profile consists of a first linear part where the temperature remains small, followed by a thin sub-layer in which it increases abruptly and barely reaches the value unity. This sub-layer is placed at a constant $y=y_{l}=2.94$.

Consequently, the following results can be derived in the large $x_{e 0}$ limit: the parameter $s_{\theta}=a_{l}^{2 / 5} x_{e 0}^{-3 / 25}$, the thickness of the thermal layer is $\epsilon_{T}=y_{l} x_{e 0}^{-7 / 10}$. Comparing the two terms in Eq. (A1), it can be obtained that the width of the thin sub-layer where the temperature experiments a sudden increase scales as $\Delta \eta \sim x_{e 0}^{-13 / 10}$, whereas the plasma velocity scales as $V \sim x_{e 0}^{-7 / 10}$.

Bearing these scaling laws into account, it is straightforward to estimate the width of the diffusive layer in the limit $\epsilon_{B} \ll \epsilon_{T}$. Forcing the diffusion term to be important in Eq. (20), the width of the diffusive layer yields $\epsilon_{B} \sim x_{e 0}^{3 / 10} / \mathrm{Le}^{1 / 2}$. The ratio of the thermal to diffusive layer thickness gives $\epsilon_{B} / \epsilon_{T} \sim x_{e 0} / \mathrm{Le}^{1 / 2}$. Then, the scaling laws obtained with this model only apply in the double limit $x_{e 0} \gg 1$ and Le $\gg x_{e 0}^{2}$, the latter being equivalent by Eq. (15) to $\beta \gg 1$.
${ }^{1}$ S. Atzeni and J. Meyer-ter Vehn, The Physics of Inertial Fusion: Beam Plasma Interaction, Hydrodynamics, Hot Dense Matter (Oxford University Press, 2004), Vol. 125.

${ }^{2}$ R. Landshoff, Phys. Rev. 76, 904 (1949).

${ }^{3}$ M. M. Basko, A. J. Kemp, and J. Meyer-ter Vehn, Nucl. Fusion 40, 59 (2000).

${ }^{4}$ I. R. Lindemuth and R. C. Kirkpatrick, Nucl. Fusion 23, 263 (1983).

${ }^{5}$ R. C. Kirkpatrick, I. R. Lindemuth, and M. S. Ward, Fusion Technol. 27, 201 (1995).

${ }^{6}$ S. A. Slutz, M. C. Herrmann, R. A. Vesey, A. B. Sefkow, D. B. Sinars, D. C. Rovang, K. J. Peterson, and M. E. Cuneo, Phys. Plasmas 17, 056303 (2010).

${ }^{7}$ S. A. Slutz and R. A. Vesey, Phys. Rev. Lett. 108, 025003 (2012).

${ }^{8}$ H. Alfvén and E. Smårs, Nature 188, 801 (1960).

${ }^{9}$ M. S. Chu, Phys. Fluids 16, 1441 (1973).

${ }^{10}$ F. S. Felber, M. A. Liberman, and A. L. Velikovich, Phys. Fluids 31, 3675 (1988).

${ }^{11}$ A. L. Velikovich, J. L. Giuliani, and S. T. Zalesak, Phys. Plasmas 22, 042702 (2015).

${ }^{12}$ R. Betti, M. Umansky, V. Lobatchev, V. N. Goncharov, and R. L. McCrory, Phys. Plasmas 8, 5257 (2001).

${ }^{13}$ J. Sanz and R. Betti, Phys. Plasmas 12, 042704 (2005).

${ }^{14}$ J. Sanz, J. Garnier, C. Cherfils, B. Canaud, L. Masse, and M. Temporal, Phys. Plasmas 12, 112702 (2005).

${ }^{15}$ S. I. Braginskii, Rev. Plasma Phys. 1, 205 (1965).

${ }^{16}$ Y. B. Zel'dovich and Y. P. Raizer, Physics of Shock Waves and High-Temperature Hydrodynamic Phenomena (Dover Publications, 2002). 



\section{Plasma-liner interface: magnetic pressure effects}

\subsection{General overview}

In this article, a theoretical analysis of magnetic pressure effects in a magnetized plasma implosion context is performed. For this purpose, the study presented in Chapter 3 has been extended to arbitrary values of the thermal-to-magnetic pressure ratio $\beta$.

Generally speaking, in the design of magnetized implosions it is not desirable to set a significant magnetic pressure level, since part of the energy used to implode would be invested into compressing the magnetic field rather than heating up the fuel. For this reason, $\beta$ values are typically high at the beginning of the implosion. However, during the implosion, the magnetic pressure increases more than the thermal pressure, and it can achieve a comparable value, that is $\beta \sim O(1)$, towards the end of it. This is simply illustrated by straightforward estimations. In an ideal adiabatic compression of a cylindrical column of plasma, thermal pressure $p$ increases as $p \sim C_{R}^{2 \gamma}$, with $C_{R}$ being the convergence ratio of the implosion and $\gamma$ the adiabatic index. The magnetic field would increase proportionally to density, that is $B \sim C_{R}^{2}$. Consequently, the thermal-to-magnetic pressure ratio $\beta$ evolves as $\beta \sim p / B^{2} \sim C_{R}^{2(\gamma-2)}$. The adiabatic index is generally smaller than 2 , and in this paper it has been taken as $\gamma=5 / 3$. Therefore, the thermal-to-magnetic pressure ratio decreases during the implosion, and finite $\beta$ effects may become important and need to be analyzed in detail, which is the aim of the article in this chapter.

The motivation to study magnetic pressure effects in a magnetized implosion context also comes from the magnetic field generation systems currently under research based on laser driven capacitor-coil assemblies [30, 71, 88]. These systems can provide very intense magnetic field values, in the order of hundreds of teslas, compared to currently technologies used in magnetized implosions such as the magneto-inertial fusion electrical discharge system MIFEDS used in OMEGA [38], which can provide up to few dozens of teslas at most. Capacitor-coil assemblies open the possibility to explore new magnetized implosion regimes where magnetic pressure effects can be important.

From a mathematical point of view, magnetic pressure effects not only come into play in the plasma momentum equation, but they also play a role in the plasma 
energy conservation through the thermoelectric Nernst and Ettingshausen effects, and Joule dissipation. As seen in Chapter 3, these effects scale as $O(1 / \beta)$ when compared to heat conduction, and they become important for finite $\beta$ values.

Magnetic pressure effects were analyzed in a similar context, the cooling of a magnetized plasma confined by an exploding metal wall, thirty years ago in Refs. [31, 32]. The authors found that the thermoelectric Nernst term, which plays the main role in the transport of magnetic field for $\beta \gg 1$, becomes less significant for $\beta \sim O(1)$, and Joule dissipation is the predominant mechanism governing the magnetic field evolution.

\subsection{Statement of the problem}

In this article, the same problem as the one analyzed in Chapter 3 is considered. A semi-infinite slab of hot magnetized plasma (hot spot) in contact with a cold dense unmagnetized plasma (liner) is set into motion by thermal conduction. The motion is considered to be subsonic, that is, small Mach number. Both plasmas are fully ionized and made of the same material (deuterium). The liner ablates into the hot spot, compressing and cooling down the hot plasma. The magnetic field is subjected to convection and diffusion. The two dimensionless governing parameters are the magnetic Lewis number $\mathrm{Le}_{\mathrm{m}}$ and the electron Hall parameter $x_{e 0}$, with the thermal-to-magnetic pressure ratio $\beta$ being related to them by Eq. (9) in the article.

Extending the analysis to arbitrary pressure ratio values is equivalent to exploring the whole $\mathrm{Le}_{\mathrm{m}}-x_{e 0}$ map. Both $\beta$ of order unity and small have been investigated, which implies $x_{e 0}^{2} \gtrsim \operatorname{Le}_{\mathrm{m}}$. Since the magnetic Lewis number is typically large in magnetized implosions, the study is restricted to high magnetization values. From a practical point of view, only the first case is of interest for magnetized implosions. Nevertheless, asymptotic results have been derived for small $\beta$ in order to better understand the magnetic pressure effects.

The qualitative description of the magnetic pressure effects is as follows. First, isobaricity no longer holds, since it is the sum of thermal plus magnetic pressures what is preserved during the motion. The presence of magnetic pressure gradients enhances mass ablation. Second, as commented in Chapter 3 , the parameter $\beta$ also represents the ratio between the thermal and magnetic diffusion layers width. Therefore, it is expected that both phenomena take place over the same length scale when $\beta \sim O(1)$. Third, the temporal derivative in the energy equation, accounting for internal plus magnetic energies variations, does not vanish, as was the case in Chapter 3. An incoming flux at infinity is therefore established that gives rise to the total energy increase. It would correspond to material convected from the center of the hot spot towards the liner. Finally, the aforementioned Nernst, Ettingshausen terms and Joule dissipation appearing in the energy conservation equation induce significant differences in the structure of the solution. More precisely, Joule dissipation becomes predominant in the region far from the liner. 


\subsubsection{Methodology}

Plasma motion and magnetic field evolution in the hot spot are governed by the ion continuity, total momentum and total energy conservation equations together with Faraday's law (induction equation) and the equation of state, Eqs. (1) - (5) in the article. A small Mach number is assumed. Momentum equation is reduced to "total isobaricity", that is, thermal plus magnetic pressure is constant and equal to what is called "total pressure" $p_{T}$ in the paper.

Taking into account magnetic pressure terms does not alter the self-similar diffusive wave structure of the solution. Accordingly, the self-similar coordinate $\eta=x / \sqrt{\kappa_{0} t}$ is introduced, with $\kappa_{0}$ being the thermal diffusivity. It should be noticed that, since both enthalpy and magnetic pressure convection need to be taken into account when establishing the energy balance, the thermal diffusivity needs to be defined with the total pressure $p_{T}$ rather than with the thermal pressure as done in Chapter 3. As a consequence, the initial magnetic field $B_{0}$ appears on the magnetic Lewis number $\mathrm{Le}_{\mathrm{m}}$. Together with $\mathrm{Le}_{\mathrm{m}}$, the second parameter governing the problem is the electron Hall parameter of the unperturbed plasma $x_{e 0}$, or, alternatively, the thermal-to-magnetic pressure ratio $\beta$ related to them by Eq. (9).

The governing system of equations can be reduced to the dimensionless ion continuity, plasma energy conservation and induction, Eqs. (12) - (14): three equations for the dimensionless ion velocity $V$, temperature $\theta$ and magnetic field $\phi$ profiles. Contrary to the large $\beta$ limit analyzed in Chapter 3 , the energy equation cannot be integrated analytically to yield an explicit expression of velocity, and the three equations need to be solved self-consistently. Still, the structure of the energy equation allows to infer that conduction, Nernst, Ettingshausen and Joule effects contribute to set the plasma into motion separately, and each of these contributions has been named as $V_{c}, V_{N}, V_{E}$ and $V_{J}$, respectively. An estimation of the order of magnitude of each term has been performed to gain insight into the predominant mechanism governing the plasma motion.

Mathematically, the problem consists of a boundary value problem of fifth order, with boundary conditions $\theta=V=\phi=0$ at $\eta=0$, and $\theta=\phi=1$ at $\eta \rightarrow$ $\infty$. It has been solved numerically designing a shooting method. An asymptotic analysis of the governing equations for $\eta \ll 1$ has been performed to elucidate the shape of temperature, velocity and magnetic field profiles close to the ablation front. The result that yields a finite ablated mass rate and zero heat flux is $\left.\theta\right|_{\eta \ll 1}=$ $s_{\theta} \eta^{2 / 5},\left.V\right|_{\eta \ll 1}=(4 / 5) s_{\theta}^{7 / 2} \eta^{2 / 5}$ and $\left.\phi\right|_{\eta \ll 1}=\operatorname{Le}_{\mathrm{m}} s_{\phi} \eta^{8 / 5}$. The parameters $s_{\theta}\left(\operatorname{Le}_{\mathrm{m}}, x_{e 0}\right)$, $s_{\phi}\left(\mathrm{Le}_{\mathrm{m}}, x_{e 0}\right)$ are positive and obtained by integrating the governing equations from the ablation front until a large enough $\eta=\eta^{*}$, and imposing the boundary conditions $\theta\left(\eta^{*}\right)=1, \phi\left(\eta^{*}\right)=1$. Again, it has been found that $\eta^{*}=8$ is sufficiently large to yield accurate results. The integration of the energy equation gives the dimensionless velocity at infinity $V_{\infty}$, which is related to the incoming flux established to increase the hot spot energy. 
Apart from the numerical computations of the equations, a simple model that allows to reduce the order of the system has been proposed. The simplification comes from noticing that the energy equation can be analytically integrated if one assumes a small value of the parameter $\zeta \equiv(2-\gamma) / \gamma(\beta+1)$. For $\gamma=5 / 3$, this parameter takes the value $\zeta=1 / 5$ at most. An expression for velocity can therefore be obtained, Eq. (31) in the article, and the system of equations is reduced to ion continuity and induction equations solely. The results given by this model have been compared to the exact problem, as discussed in the next section.

Finally, the effect of finite $\beta$ terms on the integral quantities mass ablation and magnetic flux losses per unit area in the hot spot, $m$ and $\Phi$, and thermal energy losses per unit area in the initial hot plasma, $\mathcal{E}$, has been discussed.

\subsection{Results}

The estimation of the order of magnitude of the different terms in the energy equation shows two distinguishable regions in the structure of the solution. A first one close to the liner, $\eta \ll 1$, where the plasma is relatively cold and unmagnetized, being governed by thermal conduction. The contribution $V_{c}$ becomes predominant in this region and is responsible of liner mass ablation into the hot spot. Deeper into the hot spot, a second region encompassing the bulk flow takes place. All of the terms are of the same order when $\beta \sim O(1)$, but the Joule effect $V_{J}$ becomes predominant for small $\beta$. In this limit, the whole structure of the solution, except from a layer close to the liner, is governed by magnetic field dissipation, which triggers the plasma motion through the Joule term. Both temperature and magnetic field vary in the same characteristic length. A straightforward estimation of the induction equation yields the characteristic length of the problem: $\epsilon \sim 1 / \sqrt{\mathrm{Le}_{\mathrm{m}}}$. The structure of the profiles becomes thereby insensitive to the magnetization level and is only governed by the magnetic Lewis number.

In Fig. 3(a), temperature and magnetic field profiles for a $x_{e 0}=240$ and $\beta=1$ are compared both neglecting and computing magnetic pressure terms. It is found that in the latter case, the peak of the magnetic field resulting from plasma compression is reduced. This is due to the fact that taking into account the magnetic pressure convection in the energy balance lowers the thermal diffusivity, since it is characterized with the total pressure $p_{T}$ rather than with the thermal pressure only. Consequently, the magnetic Lewis number of the problem is also reduced, that is, the plasma becomes less conductive, and the magnetic field cannot be compressed as effectively by the ablated material. Regarding the integral quantities, mass ablation is the most sensitive to the finite $\beta$ terms. It is greatly enhanced by the magnetic pressure gradients.

The structure of the solution for low $\beta$ values is shown in Fig. 4 in the paper for two different magnetic Lewis numbers. These results prove that the characteristic length 
of the solution scales indeed as $1 / \sqrt{\mathrm{Le}_{\mathrm{m}}}$. It also verifies the estimations derived: the conduction term in the energy equation $V_{c}$ dominates close to the liner, while the Joule term $V_{J}$ becomes predominant far from it. The Nernst and Ettingshausen terms, $V_{N}$ and $V_{E}$, remain relatively small. Plasma overheating in the hot spot can take place for low $\beta$ values, as show in Fig. 4(a). The magnetic energy is dissipated and transformed into internal plasma energy. Computations show that there is a critical $\mathrm{Le}_{\mathrm{m}}$ below which plasma overheating occurs and the temperature increases over its unperturbed value, which corresponds to $\mathrm{Le}_{\mathrm{m}}=10$.

Including finite $\beta$ terms in the formulation allows to study the effect of the initial magnetic field on mass ablation, thermal energy and magnetic flux losses when the initial hydrodynamic state is kept constant. They are shown in Fig. 5 in the paper as a function of the electron Hall parameter $x_{e 0}$. The pressure ratio $\beta$ is reduced when one moves towards increasing $x_{e 0}$. The three quantities decrease with magnetization until $\beta$ values of order unity are reached. From this point forward, mass ablation changes its trend and increases due to the effect of the magnetic pressure gradients. Magnetic flux losses reach a plateau, and increasing further $x_{e 0}$ no longer improves the magnetic flux conservation as effectively. The thermal energy losses, on their part, are still reduced when $\beta$ becomes of order unity. The main reason for the improvement lies in the conversion of magnetic energy into internal energy, which relieves the temperature drop caused by heat conduction.

Finally, the approximate model derived for small $\zeta$ has been compared to the exact model in Fig. 6. An explicit expression for velocity can be obtained, and it allows to infer that the magnetic field convection due to the Ettingshausen and Joule contributions to velocity, $V_{E}$ and $V_{J}$, has a diffusion structure with negative diffusivity coefficient that relieves the original diffusivity. It can be seen that the approximation is reasonably good. It underestimates magnetic flux losses and overestimates mass ablation with less than a $10 \%$ difference. The simplification treated in this model allows to include magnetic pressure effects in a straightforward way in numerical codes or in theoretical hot spot models of magnetized implosions. 



\title{
Magnetic pressure effects in a plasma-liner interface
}

\author{
F. García-Rubio a) and J. Sanz \\ E.T.S.I. Aeronáutica y del Espacio, Universidad Politécnica de Madrid, Madrid 28040, Spain
}

(Received 13 February 2018; accepted 4 April 2018; published online 24 April 2018)

\begin{abstract}
A theoretical analysis of magnetic pressure effects in a magnetized liner inertial fusion-like plasma is presented. In previous publications [F. García-Rubio and J. Sanz, Phys. Plasmas 24, 072710 (2017)], the evolution of a hot magnetized plasma in contact with a cold unmagnetized plasma, aiming to represent the hot spot and liner, respectively, was investigated in planar geometry. The analysis was made in a double limit low Mach and high thermal to magnetic pressure ratio $\beta$. In this paper, the analysis is extended to an arbitrary pressure ratio. Nernst, Ettingshausen, and Joule effects come into play in the energy balance. The region close to the liner is governed by thermal conduction, while the Joule dissipation becomes predominant far from it when the pressure ratio is low. Mass ablation, thermal energy, and magnetic flux losses are reduced with plasma magnetization, characterized by the electron Hall parameter $\omega_{e} \tau_{e}$, until $\beta$ values of order unity are reached. From this point forward, increasing the electron Hall parameter no longer improves the magnetic flux conservation, and mass ablation is enhanced due to the magnetic pressure gradients. A thoughtful simplification of the problem that allows to reduce the order of the system of governing equations while still retaining the finite $\beta$ effects is presented and compared to the exact case. Published by AIP Publishing. https://doi.org/10.1063/1.5025800
\end{abstract}

\section{INTRODUCTION}

Magnetized liner inertial fusion (MagLIF) schemes rely on imploding an axially magnetized cylindrical target. ${ }^{1}$ The presence of a magnetic field reduces the plasma thermal conductivity $^{2}$ and enhances the $\alpha$-particles energy deposition. ${ }^{3}$ This allows to relax the implosion velocity and final areal density requirements, and the high gain implosions can be conceived. $^{4,5}$

Experimental demonstration of MagLIF concept reported $2 \times 10^{12}$ thermonuclear deuterium-deuterium neutrons. ${ }^{6} \mathrm{~A}$ $2.79 \mathrm{~mm}$ outer radius target with a preimposed $10 \mathrm{~T}$ magnetic field was magnetically imploded by the pulsed power $\mathrm{Z}$ machine. The predicted implosion peak velocity was $70 \mathrm{~km} / \mathrm{s}$, lower than the typical inertial confinement fusion (ICF) implosions $(300 \mathrm{~km} / \mathrm{s})$. MagLIF design has been scaled down in size to OMEGA laser facility. ${ }^{7}$ Laser-driven MagLIF implosions consisted of $0.3 \mathrm{~mm}$ outer diameter targets with a preimposed $10 \mathrm{~T}$ magnetic field. A higher implosion velocity, $200 \mathrm{~km} / \mathrm{s}$, is needed to overcome the increase in area to volume ratio. A $2 \times$ increase in the neutron yield was reported when the magnetic field was preimposed, but the simulations expected more substantial increase for higher initial magnetic field intensities. ${ }^{8}$

An important feature of magnetized implosions is the long time that the hot plasma and the cold liner stay in contact. An understanding of the heat and magnetic flux losses mechanism through the plasma-liner interface becomes of great importance for the successful design of the implosions. In a recent publication, Velikovich and collaborators ${ }^{9}$ studied the magnetic flux and thermal energy losses in a semi-infinite magnetized plasma slab in contact with a solid

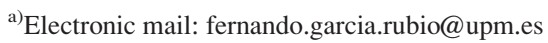

wall in a MagLIF context. Later on, in the analysis made by García-Rubio and Sanz, ${ }^{10}$ mass ablation effects were taken into account in the same problem by modeling the liner as a cold dense plasma. It was found that mass ablation promotes magnetic insulation, since thermal energy and magnetic flux losses scaled with the electron Hall parameter $\omega_{e} \tau_{e}$ as $\left(\omega_{e} \tau_{e}\right)^{-7 / 10}$ compared to $\left(\omega_{e} \tau_{e}\right)^{-1 / 2}$ reported in Ref. 9. The analysis performed in both references was carried out in the double limit small Mach number Ma $\ll 1$ and large thermal to magnetic pressure ratio $\beta \gg 1$, typical in most of the phases in a MagLIF implosion. However, it is expected that, during the compression, the fuel gets highly magnetized and the value of $\beta$ decreases. ${ }^{11}$ Pressure ratios of order unity may be achieved by the end of the implosion, especially if the magnetic field seed is generated with a capacitor-coil assembly, ${ }^{12,13}$ which can provide strong initial magnetic field values larger than $10 \mathrm{~T}$. In this case, finite $\beta$ effects should then be retained in the equations to analyze the deceleration and stagnation phases. Magnetic pressure effects were analyzed in a similar context, the cooling of a magnetized plasma confined by an exploding metal wall, thirty years ago in Refs. 14 and 15 .

The purpose of this paper is to study the magnetic pressure effects on magnetic flux losses and mass ablation in a plasma-liner interface by extending the analysis made in Ref. 10 to arbitrary values of $\beta$. Therefore we study the evolution of a hot magnetized plasma (hot spot) in contact with a cold dense unmagnetized plasma (liner) in planar geometry, as represented in the artistic scheme in Fig. 1. Retaining finite $\beta$ effects implies taking into account Nernst, Ettingshausen effects, and Joule dissipation in the energy conservation equation, which were neglected in the previous analyses. This paper is organized as follows: In Sec. II, the problem is described and the governing equations are discussed. They can be reduced to a system of three partial 


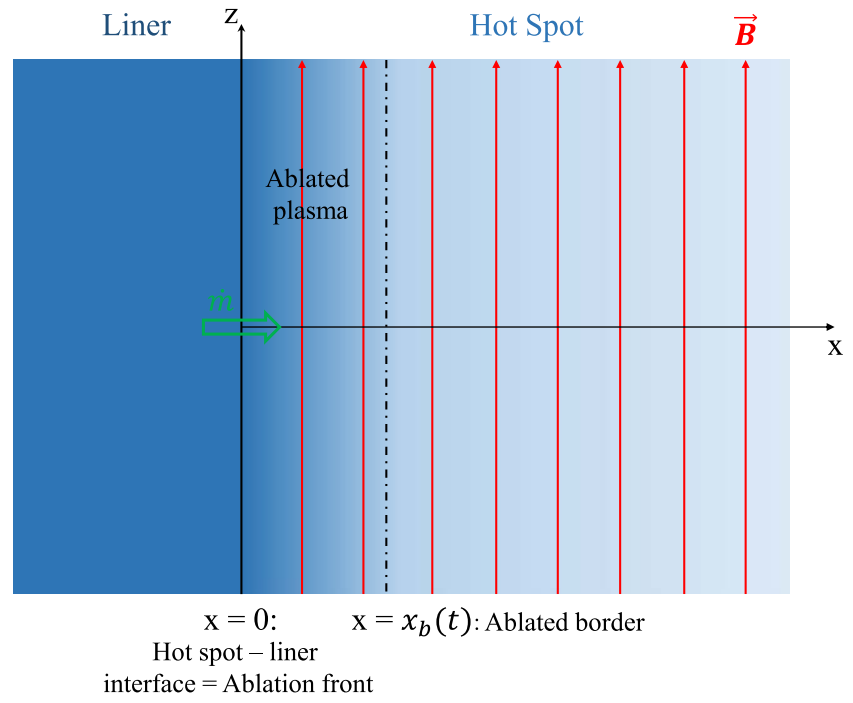

FIG. 1. Artistic scheme of the problem presented. The color gradation represents ion density. Arrows in red stand for magnetic field lines.

differential equations for temperature, velocity, and magnetic field in which the solution presents a self-similar structure. In Sec. III, the results for large $\beta$ obtained in Ref. 10 are summarized. In Sec. IV, the effect of finite $\beta$ terms is thoroughly analyzed. In Sec. V, a thoughtful simplification that retains finite $\beta$ terms but allows to simplify and reduce the order of the system of equations is described and compared to the exact case. Finally, in Sec. VI, conclusions are drawn.

\section{GOVERNING EQUATIONS}

We consider at $t=0$, a hot plasma medium at rest with a uniform temperature $T_{0}$, ion density $n_{0}$, and thermal pressure $p_{0}$ occupying the semi-infinite space $x>0$. It is initially magnetized with a uniform magnetic field $\vec{B}_{0}=B_{0} \vec{e}_{z}$. The hot plasma is in contact at $x=0$ with a cold denser unmagnetized plasma $\left(T \ll T_{0},|\vec{B}| \ll B_{0}\right)$ that extends towards $x<0$. Both plasmas are made of deuterium $(Z=1, A=2)$, and we take the adiabatic index $\gamma=5 / 3$. They are fully ionized and in mechanical equilibrium. The hot plasma would crudely represent the hot spot while the cold plasma stands for the liner. Quasi-neutrality, same ion and electron temperatures, and ideal gas hypotheses are assumed. The hot plasma is set into motion due to thermal conduction, Joule, Nernst, and Ettingshausen effects, while the magnetic field is subjected to convection and diffusion. The cold dense plasma, on the contrary, stays at rest and unmagnetized since its thermal conductivity $\chi \sim T^{5 / 2}$ is low and the magnetic diffusivity $D_{m} \sim T^{-3 / 2}$ is high (it can be considered as a magnetic isolant); neither heat flux nor electrical currents can take place in it.

Due to the geometry of the problem, the only independent variables are the streamwise direction $x$ and time $t$, the plasma ion velocity is one-dimensional $\vec{v}=v(x, t) \vec{e}_{x}$, and the magnetic field stays perpendicular to the motion $\vec{B}$ $=B(x, t) \vec{e}_{z}$. The hot plasma ion density $n$, thermal pressure $p=p_{e}+p_{i}$, temperature $T$, ion velocity $v$, and magnetic field $B$ evolution is governed by the ion continuity, plasma momentum, and energy equations neglecting electron inertia and plasma viscosity, together with Faraday's law (induction equation) and the equation of state, which for a small Mach number $\mathrm{Ma} \ll 1$ limit read

$$
\begin{gathered}
\frac{\partial n}{\partial t}+\frac{\partial}{\partial x}(n v)=0 \\
p+\frac{B^{2}}{8 \pi}=p_{T}=p_{T 0} \\
\frac{\partial}{\partial t}\left(\frac{1}{\gamma-1} p+\frac{B^{2}}{8 \pi}\right)+\frac{\partial}{\partial x}\left[\left(\frac{\gamma}{\gamma-1} p+\frac{B^{2}}{4 \pi}\right) v\right] \\
=\frac{\partial}{\partial x}[\underbrace{\chi_{\perp} \frac{\partial T}{\partial x}}_{\text {Cond. }}+\frac{c \beta_{\wedge}^{u T}}{4 \pi e n}(\underbrace{B \frac{\partial T}{\partial x}}_{\text {Nernst }}+\underbrace{T \frac{\partial B}{\partial x}}_{\text {Ettingsh. }})+\underbrace{\frac{D_{m \perp}}{4 \pi} B \frac{\partial B}{\partial x}}_{\text {Joule }}] \\
\frac{\partial B}{\partial t}+\frac{\partial}{\partial x}(v B)=\frac{\partial}{\partial x}(\underbrace{D_{m \perp} \frac{\partial B}{\partial x}}_{\text {Joule }}+\underbrace{\frac{c \beta_{\wedge}^{u T}}{e n} \frac{\partial T}{\partial x}}_{\text {Nernst }})
\end{gathered}
$$

The momentum equation (2) is reduced to total isobaricity (thermal plus magnetic): total pressure $p_{T}$ is kept constant and is given by the initial conditions $p_{T 0}=p_{0}+B_{0}^{2} / 8 \pi$. We use Braginskii's ${ }^{16}$ expressions and notations for the transport coefficients, with a constant value equal to 7 for the Coulomb logarithm. The coefficient $\chi_{\perp}$ stands for electron plus ion conductivities, $\beta_{\wedge}^{u T}$ refers to the transport coefficient for the thermoelectric Nernst and Ettingshausen effects, and $D_{m \perp}=\alpha_{\perp} c^{2} / 4 \pi e^{2} n^{2}$ is the magnetic diffusion coefficient appearing in the Joule effect.

The system of Eqs. (1)-(5) has to be completed with appropriate boundary conditions. We impose that far from the liner, $x \rightarrow \infty$, the plasma recovers the initial state $n=n_{0}, T=T_{0}$, and $B=B_{0}$. At the interface with the liner, $x=0$, we require that the plasma temperature and magnetic field be equal to the temperature and magnetic field values at the liner, that is, $T / T_{0} \rightarrow 0$ and $B / B_{0} \rightarrow 0$; and the velocity be null, $v=0$. There is a finite mass flux through the hot spot-liner interface ( $x=0$ represents an ablation front). The material from the liner is ablated and penetrates into the hot spot, pushing the fuel (initial hot plasma). The interface between the fuel and the ablated material is placed at $x_{b}(t)$ (see Fig. 1). The heat conduction losses are recycled back via this ablated material; ${ }^{17,18}$ hence, we impose zero heat flux at the liner.

\section{A. Normalization and self-similarity}

The plasma density, pressure, temperature, and magnetic field are normalized with their initial value in the hot plasma: $\rho=n / n_{0}, \psi=p / p_{0}, \quad \theta=T / T_{0}$, and $\phi=B / B_{0}$. Letting $\beta$ $=8 \pi p_{0} / B_{0}^{2}$ denote the characteristic thermal to magnetic pressure ratio, the normalized pressure and density can be linked to temperature and magnetic field profiles by means of Eqs. (2) and (5), reading 


$$
\begin{gathered}
\psi=1+\frac{1-\phi^{2}}{\beta}, \\
\rho=\frac{1+\frac{1-\phi^{2}}{\beta}}{\theta} .
\end{gathered}
$$

The thermal conductivity coefficient can be written as $\chi_{\perp}=\bar{K} T_{0}^{5 / 2} \theta^{5 / 2} \mathcal{P}_{c}\left(x_{e}\right)$, where $\bar{K} \equiv n \tau_{e} \gamma_{0} / T^{3 / 2} m_{e} \approx 2.73$ $\times 10^{20} \mathrm{eV}^{-5 / 2} \mathrm{~cm}^{-1} \mathrm{~s}^{-1}$ is a conductivity constant, and $\tau_{e}, m_{e}$ are the electron collision time and mass, respectively. The thermoelectric transport coefficient is proportional to the conductivity coefficient $\quad c \beta_{\wedge}^{u T} / e n=\left[(\gamma-1) \chi_{\perp} B / \gamma p_{0} \psi\right]$ $\mathcal{P}_{n}\left(x_{e}\right)$, while the magnetic diffusion coefficient is expressed as $\quad D_{m \perp}=\bar{D} T_{0}^{-3 / 2} \theta^{-3 / 2} \mathcal{P}_{d}\left(x_{e}\right), \quad$ with $\quad \bar{D} \equiv c^{2} m_{e} T^{3 / 2} \alpha_{0} /$ $4 \pi e^{2} n \tau_{e} \approx 2.95 \times 10^{6} \mathrm{eV}^{3 / 2} \mathrm{~cm}^{2} \mathrm{~s}^{-1}$ being a diffusivity constant. The terms $\mathcal{P}_{c}, \mathcal{P}_{n}$, and $\mathcal{P}_{d}$ account for the effect of magnetization on the transport coefficients (see Fig. 2). They are rational functions of the electron Hall parameter (electron cyclotron frequency times the electron collision time) $x_{e}=\omega_{e} \tau_{e}=\left(e B / m_{e} c\right) \tau_{e}$, and read

$$
\begin{aligned}
& \mathcal{P}_{c}\left(x_{e}\right)=\frac{\gamma_{1}^{\prime} x_{e}^{2}+\gamma_{0}^{\prime}}{\gamma_{0} \Delta_{e}}+\sqrt{\frac{2 m_{e}}{m_{i}}} \frac{2 x_{i}^{2}+2.645}{\gamma_{0} \Delta_{i}}, \\
& \mathcal{P}_{n}\left(x_{e}\right)=\frac{2 \gamma}{\gamma-1} \frac{\beta_{1}^{\prime \prime} x_{e}^{2}+\beta_{0}^{\prime \prime}}{\gamma_{1}^{\prime} x_{e}^{2}+\gamma_{0}^{\prime}+\sqrt{\frac{2 m_{e}}{m_{i}}} \frac{\Delta_{e}}{\Delta_{i}}\left(2 x_{i}^{2}+2.645\right)}, \\
& \mathcal{P}_{d}\left(x_{e}\right)=\frac{1-\frac{\alpha_{1}^{\prime} x_{e}^{2}+\alpha_{0}^{\prime}}{\Delta_{e}}}{\alpha_{0}},
\end{aligned}
$$
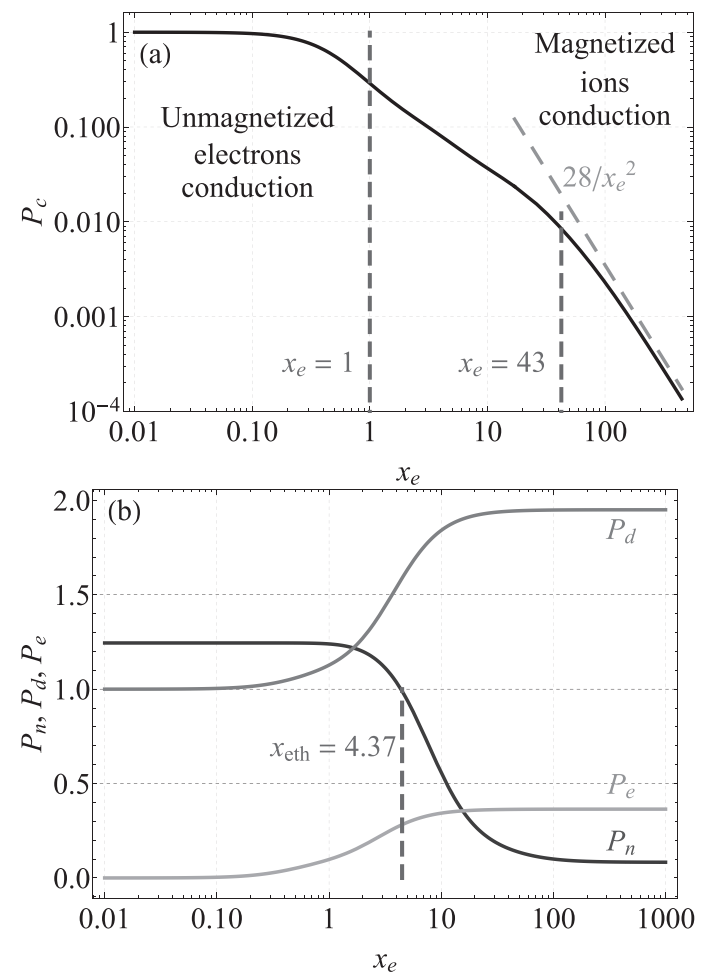

FIG. 2. Transport coefficients dependence on magnetization. with $\Delta_{e}=x_{e}^{4}+\delta_{1} x_{e}^{2}+\delta_{0} \quad$ and $\quad \Delta_{i}=x_{i}^{4}+2.70 x_{i}^{2}+0.677$, and $x_{i}=\omega_{i} \tau_{i}=\sqrt{2 m_{e} / m_{i}} x_{e}$ standing for the ion magnetization.

In an unmagnetized plasma, $x_{e} \ll 1$, thermal conduction is mainly due to electrons because of their small mass and $\mathcal{P}_{c}=1$. However, the electrons get magnetized for lower magnetic field intensities compared to the ions $\left(x_{e}=1\right.$ with respect to $x_{e}=\sqrt{m_{i} / 2 m_{e}} \approx 43$ ), and when electron conduction is suppressed, the ions carry the heat transport. For large magnetization values $\left(x_{e}>43\right)$, the ions also get magnetized and the thermal conductivity decreases asymptotically as $\mathcal{P}_{c} \approx 28 / x_{e}^{2}$.

The electron Hall parameter anywhere in the hot plasma, $x_{e}$, can be written as a function of its value far from the liner and the dimensionless temperature and magnetic field profiles

$$
x_{e}=x_{e 0} \frac{\phi \theta^{5 / 2}}{1+\frac{1-\phi^{2}}{\beta}} .
$$

We define the magnetic Lewis number Le as the ratio between thermal and magnetic diffusivities, ${ }^{9,10}$ defined, on their part, as $\kappa_{0}=(\gamma-1) \bar{K} T_{0}^{7 / 2} / \gamma p_{T}$ and $\nu_{m 0}=\bar{D} T_{0}^{-3 / 2}$, respectively. It is related to the pressure ratio $\beta$ and electron Hall parameter $x_{e 0}$ through

$$
\text { Le }=\frac{\kappa_{0}}{\nu_{m 0}}=\frac{\gamma-1}{8 \gamma} \frac{\gamma_{0}}{\alpha_{0}} \frac{\beta^{2} x_{e 0}^{2}}{\beta+1} \approx 0.31 \frac{\beta^{2} x_{e 0}^{2}}{\beta+1} .
$$

Since the initial value problem proposed lacks a characteristic length and velocity, the solution to Eqs. (1)-(5) is sought under the form of a self-similar diffusive wave. ${ }^{19} \mathrm{We}$ introduce, consequently, the independent self-similar variable

$$
\eta=\frac{x}{\sqrt{\kappa_{0} t}}(\eta \geq 0)
$$

Consistently, the plasma ion velocity is scaled self-similarly

$$
V(\eta)=2 \sqrt{\frac{t}{\kappa_{0}}} v(x, t) .
$$

The dimensionless ion continuity, plasma energy, and induction equations take the form

$$
\begin{gathered}
(V-\eta) \frac{\mathrm{d} \rho}{\mathrm{d} \eta}+\rho \frac{\mathrm{d} V}{\mathrm{~d} \eta}=0 \\
\frac{\mathrm{d}}{\mathrm{d} \eta}\left[\left(1-\frac{2-\gamma}{\gamma(\beta+1)} \phi^{2}\right) V\right] \\
=\frac{\mathrm{d}}{\mathrm{d} \eta}\left(V_{c}+V_{N}+V_{E}+V_{J}\right)-\frac{2-\gamma}{\gamma(\beta+1)} \eta \frac{\mathrm{d} \phi^{2}}{\mathrm{~d} \eta} \\
-\eta \frac{\mathrm{d} \phi}{\mathrm{d} \eta}+\frac{\mathrm{d}}{\mathrm{d} \eta}\left[\left(V-\frac{2 \mathcal{P}_{n} \mathcal{P}_{c}}{1-\frac{\phi^{2}}{\beta+1}} \theta^{5 / 2} \frac{\mathrm{d} \theta}{\mathrm{d} \eta}\right) \phi\right] \\
=\frac{2}{\mathrm{Le}} \frac{\mathrm{d}}{\mathrm{d} \eta}\left(\mathcal{P}_{d} \theta^{-3 / 2} \frac{\mathrm{d} \phi}{\mathrm{d} \eta}\right)
\end{gathered}
$$


with

$$
\begin{aligned}
V_{c} & =2 \mathcal{P}_{c} \theta^{5 / 2} \frac{\mathrm{d} \theta}{\mathrm{d} \eta}, \\
V_{N} & =\frac{4(\gamma-1)}{\gamma(\beta+1)} \frac{\mathcal{P}_{c} \mathcal{P}_{n}}{1-\frac{\phi^{2}}{\beta+1}} \phi^{2} \theta^{5 / 2} \frac{\mathrm{d} \theta}{\mathrm{d} \eta}, \\
V_{E} & =\frac{4(\gamma-1)}{\gamma(\beta+1)} \frac{\mathcal{P}_{c} \mathcal{P}_{n}}{1-\frac{\phi^{2}}{\beta+1}} \theta^{7 / 2} \phi \frac{\mathrm{d} \phi}{\mathrm{d} \eta}, \\
V_{J} & =\frac{4(\gamma-1)}{\gamma(\beta+1)} \frac{1}{\mathrm{Le}} \mathcal{P}_{d} \theta^{-3 / 2} \phi \frac{\mathrm{d} \phi}{\mathrm{d} \eta},
\end{aligned}
$$

standing for conduction, Nernst, Ettingshausen, and Joule effects contribution to ion velocity, respectively. The last term on the right hand side of Eq. (13) is due to the temporal variation of the total energy (thermal plus magnetic), which is zero in a large $\beta$ limit.

By making use of the equation of state (7), the problem is reduced to a system of three highly coupled nonlinear ordinary differential equations of fifth order for $\theta, V$, and $\phi$ : Eqs. (12)-(14). We remind the boundary conditions: $\theta=V$ $=\phi=0$ at $\eta=0$, and $\theta=\phi=1$ at $\eta=\infty$. As discussed in Ref. 10, the ablated border travels at a constant $\eta_{b}=x_{b} /$ $\sqrt{\kappa_{0} t}$ and is given by the equation $V\left(\eta_{b}\right)=\eta_{b}$. According to this relation and Eq. (13), $\eta_{b}$ accounts for the sum of heat flux, thermoelectric and Joule effects at the ablated border plus terms of the order of $O((2-\gamma) / \gamma(\beta+1))$.

The information about the initial state $\left(n_{0}, T_{0}, B_{0}\right)$ is thereby condensed into two free parameters, which can be any two of $\left(x_{e 0}\right.$, Le, $\left.\beta\right)$, being the third one determined by Eq. (9). It is remarkable to notice that since both enthalpy and magnetic pressure convection needs to be taken into account when establishing the energy balance, the thermal diffusivity needs to be defined with the total pressure $p_{T}$. As a consequence, the initial magnetic field $B_{0}$ appears on the Lewis number. For this reason, in order to compare solutions corresponding to initial conditions that only differ in the magnetic field, it is useful to define another dimensionless number based uniquely on the hydrodynamic variables $n_{0}$ and $T_{0}$ : Lh $=\kappa_{0_{H}} / \nu_{m 0}$, with $\kappa_{0_{H}}=(\gamma-1) \bar{K} T_{0}^{7 / 2} / \gamma p_{0}$ being the thermal diffusivity based on the thermal pressure $p_{0}$. It is related to the former Lewis number by $\operatorname{Lh}=\operatorname{Le}(\beta+1) / \beta$. Two cases with the same initial hydrodynamic variables will have the same Lh and $\kappa_{0_{H}}$. Notice that Lh corresponds to the magnetic Lewis number of the problem defined in Ref. 10, where the magnetic pressure terms are neglected. The following numerical relations are derived for comparison purposes:

$$
\begin{aligned}
x_{e 0} & =0.087\left(\frac{T_{0}}{100 \mathrm{eV}}\right)^{3 / 2}\left(\frac{n_{0}}{10^{21} \mathrm{~cm}^{-3}}\right)^{-1}\left(\frac{B_{0}}{10 \mathrm{~T}}\right), \\
\mathrm{Lh} & =1.85\left(\frac{T_{0}}{100 \mathrm{eV}}\right)^{4}\left(\frac{n_{0}}{10^{21} \mathrm{~cm}^{-3}}\right)^{-1}, \\
\beta & =805\left(\frac{T_{0}}{100 \mathrm{eV}}\right)\left(\frac{n_{0}}{10^{21} \mathrm{~cm}^{-3}}\right)\left(\frac{B_{0}}{10 \mathrm{~T}}\right)^{-2} .
\end{aligned}
$$

As a consequence of the total energy variation in time, the last term in the energy equation (13), an incoming flux at infinity, is established. Letting $v_{\infty}$ denote the plasma velocity at $x \rightarrow \infty$ and $V_{\infty}$ its normalized counterpart, Eq. (13) can be integrated in the whole hot spot region $0 \leq \eta<\infty$, giving the next relation

$$
V_{\infty}=-\frac{\frac{2-\gamma}{\gamma(\beta+1)}}{1-\frac{2-\gamma}{\gamma(\beta+1)}} \int_{0}^{\infty}\left(1-\phi^{2}\right) \mathrm{d} \eta .
$$

Numerical resolution of the system performed for a wide scan of the parameters $\left(\right.$ Le, $\left.x_{e 0}\right)$ found the integral $\int_{0}^{\infty}\left(1-\phi^{2}\right) \mathrm{d} \eta$ to be always positive. This entails an incoming mass flux $\left(V_{\infty}<0\right)$ from the center of the hot spot towards the liner that increases the total energy of the hot spot defined in $0 \leq x$ $<\infty$. Notice that this mass flux becomes negligible for small values of the parameter $2-\gamma / \gamma(\beta+1)$. It is remarkable that in Ref. 9, the authors also reported an incoming mass flux towards the liner, but it was originated by a different reason. There, the analysis is performed in a large $\beta$ limit, so the thermal pressure is homogeneous and the total energy is conserved, contrary to our case. However, in that study, the liner is treated as a solid wall (no ablation), and consistently there is a finite heat flux from the hot spot towards the liner at $x=0$. Therefore, an enthalpy flux coming from infinity needs to be established to overcome heat losses and restore isobaricity.

\section{B. Analysis of the region close to the liner}

An asymptotic analysis of the governing equations (12)-(14) for $\eta \ll 1$ has been performed in order to elucidate the shape of temperature, velocity, and magnetic field profiles close to the liner. The result that yields a finite ablated mass rate and zero heat flux is

$$
\begin{gathered}
\left.\theta\right|_{\eta \ll 1}=s_{\theta} \eta^{2 / 5},\left.\quad \phi\right|_{\eta \ll 1}=s_{\phi} \operatorname{Le}^{8 / 5}, \\
\left.\rho\right|_{\eta \ll 1}=\frac{\beta+1}{\beta} \frac{1}{s_{\theta} \eta^{2 / 5}},\left.\quad V\right|_{\eta \ll 1}=s_{V} \eta^{2 / 5}=\frac{4}{5} s_{\theta}^{7 / 2} \eta^{2 / 5} .
\end{gathered}
$$

The parameters $s_{\theta}\left(\mathrm{Le}, x_{e 0}\right)$ and $s_{\phi}\left(\mathrm{Le}, x_{e 0}\right)$ are obtained by solving the complete problem with the boundary conditions far from the liner $\theta(\infty)=\phi(\infty)=1$.

According to these profiles, the main contribution to ion velocity in the region close to the liner corresponds to thermal conduction $V_{c} \sim \eta^{2 / 5}$, since the other terms in Eq. (13) are smaller of the order $V_{N} \sim V_{E} \sim \eta^{18 / 5}, V_{J} \sim \eta^{8 / 5}$. Therefore, the region close to the liner is dominated by thermal conduction, and the plasma dynamics therein are similar to the large $\beta$ case analyzed in Ref. 10 .

As outlined in the same reference, the governing equations admit another solution, which corresponds to zero ablated mass rate and finite heat flux at the liner: $\theta=s_{\theta} \eta^{2 / 7}$, $\phi=\operatorname{Le} s_{\phi} \eta^{10 / 7}, \quad \rho=(\beta+1) / \beta s_{\theta} \eta^{2 / 5}, V=-(2 / 5) \eta \quad$ for $\eta \ll 1$. This solution, treated in Ref. 9 for a large $\beta$ limit, will not be considered in this paper.

\section{Mass ablation, thermal energy, and magnetic flux losses}

We define the mass increase $m$ (expressed in ion particles per unit area) and magnetic flux losses $\Phi$ per unit area in the hot spot as 


$$
\begin{aligned}
& m=\int_{0}^{\infty}\left(n-n_{0}\right) \mathrm{d} x, \\
& \Phi=\int_{0}^{\infty}\left(B_{0}-B\right) \mathrm{d} x .
\end{aligned}
$$

Another quantity of interest is the thermal energy losses $\mathcal{E}$ per unit area in the initial hot plasma

$$
\mathcal{E}=\int_{0}^{\infty} \frac{p_{0}}{\gamma-1} \mathrm{~d} x-\int_{x_{b}}^{\infty} \frac{p}{\gamma-1} \mathrm{~d} x .
$$

Integrating in space Eqs. (1) and (4), the time derivative of $m$ and $\Phi$ can be related to the mass ablation rate and magnetic diffusion at the liner $(x=0)$, reading

$$
\frac{\mathrm{d} m}{\mathrm{~d} t}=\left.n v\right|_{x=0}-n_{0} v_{\infty}, \quad \frac{\mathrm{d} \Phi}{\mathrm{d} t}=\left.D_{m \perp} \frac{\partial B}{\partial x}\right|_{x=0}+B_{0} v_{\infty} .
$$

Formally speaking, there is mass and magnetic field advection due to $v_{\infty}$ into our system defined in $0 \leq x<\infty$. However, in a physical picture, it corresponds to mass and magnetic field coming from the center of the hot spot into the liner, and therefore it does not represent an effective hot spot mass or magnetic flux increase, and will not be taken into account. The time derivative of mass and magnetic flux can therefore be expressed in terms of the parameters $s_{\theta}, s_{\phi}$ as

$$
\begin{gathered}
\frac{\mathrm{d} m}{\mathrm{~d} t}=\frac{2 n_{0}}{5} \frac{\beta+1}{\beta} \sqrt{\frac{\kappa_{0}}{t} s_{\theta}^{5 / 2},} \\
\frac{\mathrm{d} \Phi}{\mathrm{d} t}=\frac{8 B_{0}}{5} \sqrt{\frac{\kappa_{0}}{t}} \frac{s_{\phi}}{s_{\theta}^{3 / 2}} .
\end{gathered}
$$

Since the parameters $s_{\theta}, s_{\phi}$ do not depend on time, these equations can be integrated yielding

$$
\begin{gathered}
m=\frac{4 n_{0}}{5} \frac{\beta+1}{\beta} \sqrt{\kappa_{0} t} s_{\theta}^{5 / 2}, \\
\Phi=\frac{16 B_{0}}{5} \sqrt{\kappa_{0} t} \frac{s_{\phi}}{s_{\theta}^{3 / 2}} .
\end{gathered}
$$

The thermal energy losses, on their part, can be expressed by the use of total isobaricity (2) as

$$
\mathcal{E}=\frac{p_{0}}{\gamma-1} \sqrt{\kappa_{0} t}\left(\eta_{b}-\int_{\eta_{b}}^{\infty} \frac{1-\phi^{2}}{\beta} \mathrm{d} \eta\right) .
$$

The sum of heat flux, thermoelectric and Joule effects at the ablated border, represented by $\eta_{b}$, transfers thermal energy from the fuel into the ablated material, heating it up. This energy loss is relieved by the magnetic energy dissipated and transformed into thermal energy, and given by the integral appearing in (26).

It is useful to express mass ablation, thermal energy, and magnetic flux losses normalized with hydrodynamic variables only

$$
\frac{m}{n_{0} \sqrt{\kappa_{0_{H}} t}}=\frac{4}{5} s_{\theta}^{5 / 2} \sqrt{\frac{\beta+1}{\beta}},
$$

$$
\begin{gathered}
\frac{\mathcal{E}(\gamma-1)}{p_{0} \sqrt{\kappa_{0_{H}} t}}=\left(\eta_{b}-\int_{\eta_{b}}^{\infty} \frac{1-\phi^{2}}{\beta} \mathrm{d} \eta\right) \sqrt{\frac{\beta}{\beta+1}}, \\
\frac{\Phi}{B_{0} \sqrt{\kappa_{0_{H}} t}}=\frac{16}{5} \frac{s_{\phi}}{s_{\theta}^{3 / 2}} \sqrt{\frac{\beta}{\beta+1}} .
\end{gathered}
$$

\section{REMINDER OF LARGE $\beta$ RESULTS}

In this section, we summarize the results derived in Ref. 10. In a large $\beta$ limit, Eq. (13) can be integrated, yielding $V=V_{c}=2 \mathcal{P}_{c} \theta^{5 / 2} \mathrm{~d} \theta / \mathrm{d} \eta$. The hot plasma is cooled down and set into motion by thermal conduction. Mass ablated at the liner pushes the plasma towards the center of the hot spot. The magnetic field is subjected to diffusion and convection due to plasma motion and the Nernst term. The latter advects the magnetic field in the heat flux direction.

In the unmagnetized case $\left(x_{e 0} \ll 1\right)$, the parameter $s_{\theta}$ takes the value $s_{\theta}=1.045$ independently of the Lewis number. Nernst advection prevails over advection due to plasma motion, and the magnetic field is convected into the ablated material and pushed towards the liner. For large Le, the magnetic field accumulates close to the liner and is diffused in a thin layer. Letting $\epsilon_{B}$ be the width of the magnetic field diffusion layer, it scales as $\epsilon_{B} \approx 1.42 / \sqrt{\mathrm{Le}}$. The parameter $s_{\phi}$ decreases with the Lewis number until it reaches the lower limit value $s_{\phi}=0.015$ for large Le, and cannot be further reduced. The Nernst term acts therefore as an engine that pushes the magnetic field against the liner and enhances its diffusion even if the plasma is highly conductive (large Le).

When the hot plasma is magnetized, thermal conduction is reduced. Layers of hot plasma stay closer to the liner compared to the unmagnetized solution, and the characteristic length of temperature variation is reduced. Letting $\epsilon_{T}$ be the width of this thermal layer, it scales as $\epsilon_{T} \sim 1 / x_{e 0}^{7 / 10}$ for large $x_{e 0}$ values. The Nernst term, suppressed in the magnetized plasma, is only active in the unmagnetized region very close to the liner, and its effect enhancing magnetic diffusion is reduced. In the high magnetization limit, the magnetic diffusion layer scales as $\epsilon_{B} \sim x_{e 0}^{3 / 10} / \sqrt{\mathrm{Le}}$. The ratio between the two widths reads $\epsilon_{T} / \epsilon_{B} \sim \sqrt{\mathrm{Le}} / x_{e 0} \sim \sqrt{\beta} \gg 1$ : in the large $\beta$ limit, the magnetic field stays of order unity in the thermal characteristic length, and diffuses in a much thinner layer. Mass ablation, thermal energy, and magnetic flux losses are reduced with magnetization, and tend asymptotically to $m / n_{0} \sqrt{\kappa_{0_{H}} t}=1.62 / x_{e 0}^{3 / 10}, \quad(\gamma-1) \mathcal{E} / p_{0} \sqrt{\kappa_{0_{H}} t}=1.75 / x_{e 0}^{7 / 10}$, and $\Phi / B_{0} \sqrt{\kappa_{0_{H}} t}=0.15 / x_{e 0}^{7 / 10}$.

\section{RESULTS FOR FINITE AND SMALL $\beta$}

When the thermal to magnetic pressure ratio $\beta$ is of order unity or small, thermal pressure is no longer homogeneous, and the Nernst, Ettingshausen, and Joule effects play an important role in the energy balance (13). The width of the diffusion layer becomes comparable to the thermal layer, $\epsilon_{T} \sim \epsilon_{B}$, and will simply be denoted by $\epsilon$.

As discussed in Subsection II B, the region close to the liner is dominated by thermal conduction. Deeper into the 
hot spot, where $\theta$ and $\phi$ are assumed to be of order unity, we can estimate each of the contributions to velocity, yielding

$$
V_{c} \sim \frac{\mathcal{P}_{c}}{\epsilon}, \quad V_{N} \sim V_{E} \sim \frac{\mathcal{P}_{c}}{\epsilon}, \quad V_{J} \sim \frac{1}{\operatorname{Le} \epsilon} .
$$

The Nernst and Ettingshausen terms are of the same order of magnitude as thermal conduction. Comparing these terms to the Joule effect, we obtain

$$
\frac{V_{c}}{V_{J}} \sim \mathcal{P}_{c} \operatorname{Le} \sim \mathcal{P}_{c} x_{e 0}^{2} \beta^{2} .
$$

The function $\mathcal{P}_{c}\left(x_{e 0}\right) x_{e 0}^{2}$ is a monotonically increasing function which tends asymptotically to 28 when $x_{e 0} \rightarrow \infty$. We can deduce that, far from the liner, all terms are of the same order of magnitude when $\beta \sim O(1)$, but the Joule effect $V_{J}$ becomes predominant for small $\beta$. In this limit, we can therefore identify two different regions: one close to the liner dominated by thermal conduction, and the other placed deeper into the hot spot governed by Joule dissipation. A straightforward estimation of the terms in Eq. (14) suggests $\eta \mathrm{d} \phi / \mathrm{d} \eta \sim O(1),(2 / \mathrm{Le}) \mathrm{d}\left(\mathcal{P}_{d} \theta^{-3 / 2} \mathrm{~d} \phi / \mathrm{d} \eta\right) / \mathrm{d} \eta \sim 1 / \operatorname{Le} \epsilon^{2}$. Equating these two terms, we obtain $\epsilon \sim 1 / \sqrt{\mathrm{Le}}$. The scale length of the problem becomes thereby independent of plasma magnetization level in a low pressure ratio limit.

\section{A. Temperature, velocity, and magnetic field profiles}

The governing equations (12)-(14) have been numerically solved in order to investigate the finite $\beta$ effects. In order to show a realistic case that could take place in laserdriven MagLIF, we assume that at the initial state of the implosion, the temperature is $T_{i}=100 \mathrm{eV}$ and fuel density $\rho_{i}$ $=2.7 \mathrm{mg} / \mathrm{cc}$. We take as initial magnetic field $B_{i}=100 \mathrm{~T}$, which could be provided by a laser-driven capacitor-coil assembly. The electron Hall parameter and pressure ratio would therefore be $x_{e 0}=1.07$ and $\beta=6.5$, respectively. If the implosion is assumed to be ideal, the variables will evolve as $T=T_{i} C_{R}^{2(\gamma-1)}, \rho=\rho_{i} C_{R}^{2}$, and $B=B_{i} C_{R}^{2}$, with $C_{R}$ being the convergence ratio. For a convergence ratio $C_{R}=15$ and $\gamma=5 / 3$, we would have by the end of the implosion $T=3.7 \mathrm{keV}, B=225 \mathrm{MG}, \mathrm{x}_{\mathrm{e} 0}=241$, and $\beta=1.07$.

In Fig. 3(a), temperature and magnetic field profiles are shown for $x_{e 0}=240$ and $\beta=1$, which give $\mathrm{Lh}=1.8 \times 10^{4}$. The profiles taking into account finite $\beta$ effects $\left(\theta_{\beta}, \phi_{\beta}\right)$ are plotted together with the solution given by the governing equations derived in Ref. 10 for the same initial conditions $\left(\theta_{\mathrm{no} \beta}, \phi_{\mathrm{no} \beta}\right)$, where these effects have been neglected. In order to compare both the set of profiles, the horizontal axis corresponds to $\eta_{H}=x / \sqrt{\kappa_{0_{H}} t}$. The magnetic field is compressed by the ablated material, and it presents a peak and is diffused in a thick layer, whose width is comparable to the characteristic length scale of temperature $\left(\epsilon_{T}\right)$. It can be seen that the peak of the magnetic field is reduced and takes place closer to the liner when finite $\beta$ terms are computed. Taking into account the magnetic pressure convection in the energy balance lowers the thermal diffusivity, therefore, the scale length of the solution decreases and the Lewis number of the problem is reduced from $\mathrm{Le}=\mathrm{Lh}=1.8 \times 10^{4}$ to
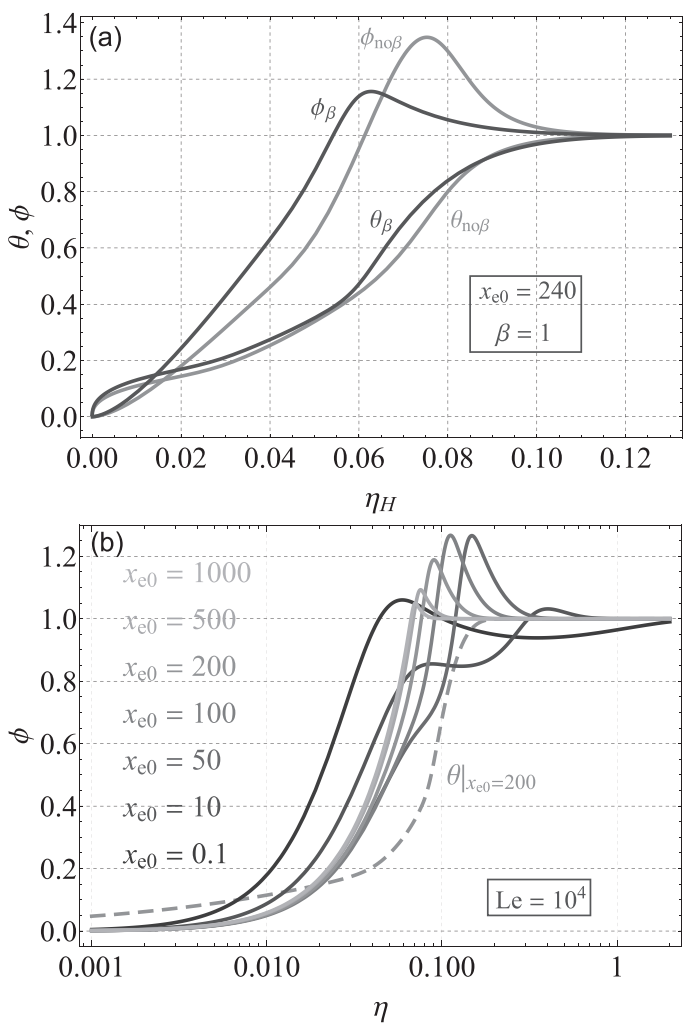

FIG. 3. (a) Normalized temperature and magnetic field profiles for $x_{e 0}=240, \beta=1$, which gives $\mathrm{Lh}=1.8 \times 10^{4}$ and $\mathrm{Le}=9 \times 10^{3}$. The profiles in black $\left(\theta_{\beta}, \phi_{\beta}\right)$ are given by the governing equations (12)-(14), while the profiles in gray $\left(\theta_{\mathrm{no} \beta}, \phi_{\mathrm{no} \beta}\right)$ correspond to the solution of the problem neglecting finite $\beta$ effects [governing equations (19) and (20) in Ref. 10] computed for the same initial conditions. The horizontal axis corresponds to the dimensionless variable $\eta_{H}=x / \sqrt{\kappa_{0_{H}} t}$. (b) Normalized magnetic field profiles for $\mathrm{Le}=10^{4}, x_{e 0}=0.1,10,50,100,200,500,1000$. In each case, the parameter $\beta$ is $3 \times 10^{6}, 325,14,4,1.39,0.43,0.19$, respectively. The temperature profile $\theta$ is shown in dashed lines for the $x_{e 0}=200, \beta=1.39$ case.

Le $=9 \times 10^{3}$. The plasma becomes less conductive, and cannot be compressed as effectively by the ablated material; hence, the peak of the magnetic field profile is reduced. The normalized mass ablation $m / n_{0} \sqrt{\kappa_{0_{H}} t}$, thermal energy $(\gamma-1) \mathcal{E} / p_{0} \sqrt{\kappa_{0_{H}} t}$, and magnetic flux losses $\Phi / B_{0} \sqrt{\kappa_{0_{H}} t}$ give $0.56,0.045$ and 0.0326 when finite $\beta$ terms are taken into account, and $0.35,0.044$ and 0.0315 when they are not. Mass ablation is more sensitive to finite $\beta$ effects than the losses, mostly due to the effect of magnetic pressure gradients, which enhances the convection of cold plasma layers from the liner in order to restore total isobaricity.

Magnetic field profiles for the same Le $=10^{4}$ and varying $x_{e 0}$ are shown in Fig. 3(b). As the electron Hall parameter is increased, the corresponding $\beta$ is smaller. It becomes of order unity, $\beta=1.39$, for $x_{e 0}=200$. Higher magnetization values, although unlikely to be achieved in MagLIF, are shown for comparison purposes. When the magnetization is low, the magnetic field is convected by the Nernst term towards the liner. It penetrates into the ablated plasma, accumulates close to the liner, and presents a peak there. This peak is reduced when the plasma is magnetized, since the Nernst term gets confined to the unmagnetized plasma region close to the liner and becomes less effective. ${ }^{10}$ A second peak takes place in the magnetized plasma due to the 
compression exerted by the ablated material. The diffusion layer widens when $x_{e 0}$ increases as long as $\beta$ keeps a large value according to the scaling $\epsilon_{B} \sim x_{e 0}^{3 / 10} / \sqrt{\text { Le }}$ derived in Ref. 10. However, when $\beta$ becomes of order unity, the diffusive layer shortens for increasing $x_{e 0}$ until it gets of order $\epsilon_{B} \sim 1 / \sqrt{\mathrm{Le}}$. The diffusion and thermal layers get of the same order of magnitude, and magnetic diffusion becomes important in all the domains. This is consistent with the results derived in Refs. 14 and 15, where it was found that the thermoelectric (Nernst) phenomena, which play the main role in the transport of magnetic field for $\beta \gg 1$, become less significant for $\beta \sim 1$. The two peaks are reduced until they both vanish when $\beta$ is small (large $x_{e 0}$ ). Numerical solutions obtained when the pressure ratio $\beta$ is further decreased $\left(x_{e 0}\right.$ raises) while maintaining a constant Le show that the temperature and magnetic field profiles no longer change appreciably. The parameters $s_{\theta}$ and $s_{\phi}$ tend to a constant value, which indicates that the system gets independent of the magnetization level and is only governed by the Lewis number.

In order to shed more light on the effect of the finite $\beta$ terms, temperature, velocity, and magnetic field profiles for a small $\beta=0.1$, and $\mathrm{Le}=1$ or $\mathrm{Le}=100$, are plotted in Figs. 4(a) and 4(b). The self-similar variable $\eta$ (abscissa) has been normalized using the Lewis number, proving that the scale length of the solution corresponds to $\epsilon \sim 1 / \sqrt{\mathrm{Le}}$ when $\beta$ is small. The different contributions to velocity have been depicted in Fig. 4(a). As derived in the estimations
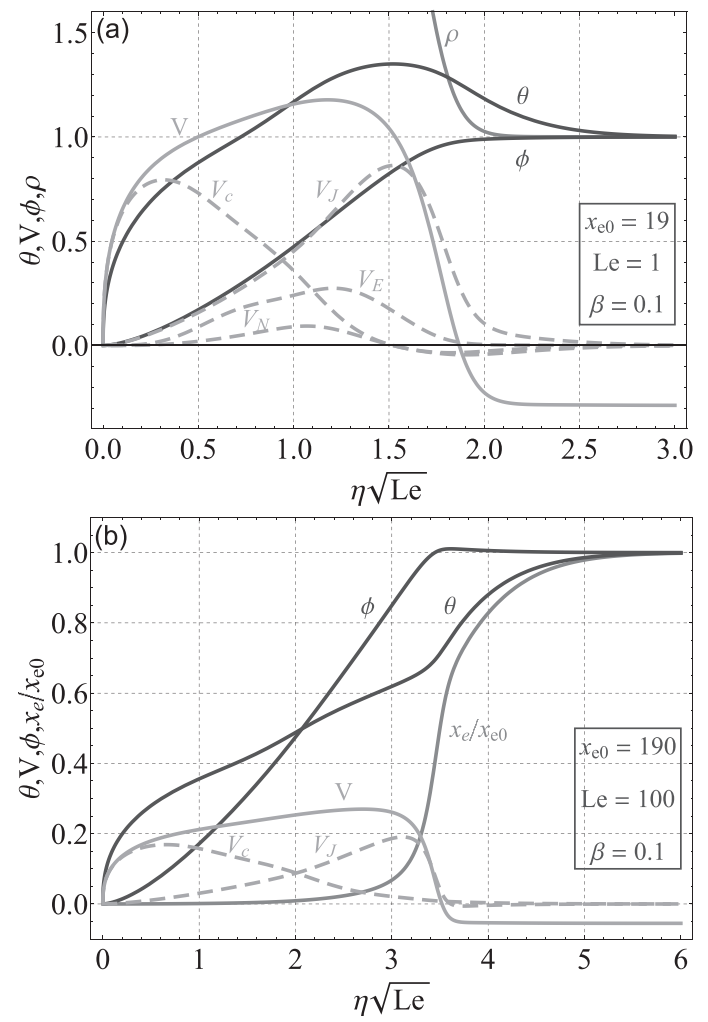

FIG. 4. Normalized temperature $\theta$, velocity $V$, and magnetic field $\phi$ profiles for $\beta=0.1$ and (a) $x_{e 0}=19$, (b) $x_{e 0}=190$, which gives Le $=1, \mathrm{Le}=100$, respectively. Normalized density $\rho$ and different contributions to velocity $V_{c}, V_{N}, V_{E}$, and $V_{J}$ are plotted in gray solid line and light gray dashed lines, respectively, in (a); while the magnetization normalized with the initial magnetization is plotted in gray solid line in (b). performed at the beginning of this section, two different regions can be identified: a first one close to the liner dominated by conduction $\left(V_{c}\right)$, and a second one further into the hot spot dominated by Joule diffusion $\left(V_{J}\right)$. The Nernst and Ettingshausen effects in the energy equation $\left(V_{N}, V_{E}\right)$ remain relatively small. The velocity profile undergoes a sudden decrease where the magnetic field gets close to one, that is $1-\phi \sim O(\beta)$. In this outer region, the estimations derived previously break due to the smallness of the magnetic field derivative, and conduction and Joule dissipation become of the same order. Due to the smallness of $\beta$, the temperature profile, which obeys the equation of state (7), is very sensitive to the evolution of the magnetic field in this region, where density is still unperturbed, and consequently $\theta \approx 1$ $+\left(1-\phi^{2}\right) / \beta$. Depending on the Lewis number, Joule dissipation may overcome magnetic field convection in this region, $\phi$ is reduced and temperature is increased over its initial value, $\theta>1$, due to Joule heating. This takes place for relatively low Le, as seen in Fig. 4(a). However, when the Lewis is larger, Fig. 4(b), magnetic field convection compresses it slightly, $\phi$ gets higher than unity and as a consequence, temperature experiments a steep decrease. Computations show that there is a critical Lewis number below which the temperature profile can achieve values above unity when $\beta$ is small, which corresponds to $\mathrm{Le}=10$. Typically, Lewis numbers are high in MagLIF plasmas, so this overheating is not expected to happen.

The magnetization profile has been depicted in Fig. 4(b). It can be seen that it is highly reduced as soon as the magnetic field departs from unity, $1-\phi \sim O(1)$. The main reason for this lies in the enhancement of mass ablation promoted by the magnetic pressure gradients. Normalized density becomes of order $\rho \sim 1 / \beta$, and the magnetization is consequently reduced to $x_{e} \sim x_{e 0} \beta$.

\section{B. Mass ablation, thermal energy, and magnetic flux losses}

It is interesting to analyze the effect of the initial magnetic field on mass ablation, thermal energy, and magnetic flux losses when the initial hydrodynamic state is kept constant. For this purpose, these quantities are computed in Fig. 5 for increasing magnetization maintaining the same Lh. The pressure ratio $\beta$ is reduced when one moves towards increasing $x_{e 0}$. The scaling laws obtained for an infinite $\beta$ limit in Ref. 10 are depicted for comparison. The three quantities are reduced, following the same trend as in Fig. 6 in Ref. 10, until $\beta$ values of order unity are reached. From this point forward, mass ablation is increased due to the effect of the magnetic pressure gradients and magnetic flux losses reach a plateau. Therefore, increasing the magnetization after $\beta$ becomes of order unity no longer improves the magnetic flux conservation. It is not the case for thermal insulation. Although it is found that $\eta_{b}$ increases with magnetization after $\beta$ becomes of order unity, the conversion of magnetic energy into thermal energy and the reduction of the thermal diffusivity $\kappa_{0}$ with the magnetic pressure relieve this effect, resulting in an overall improvement of the thermal insulation even when $\beta$ gets of order unity, Fig. 5(b). 

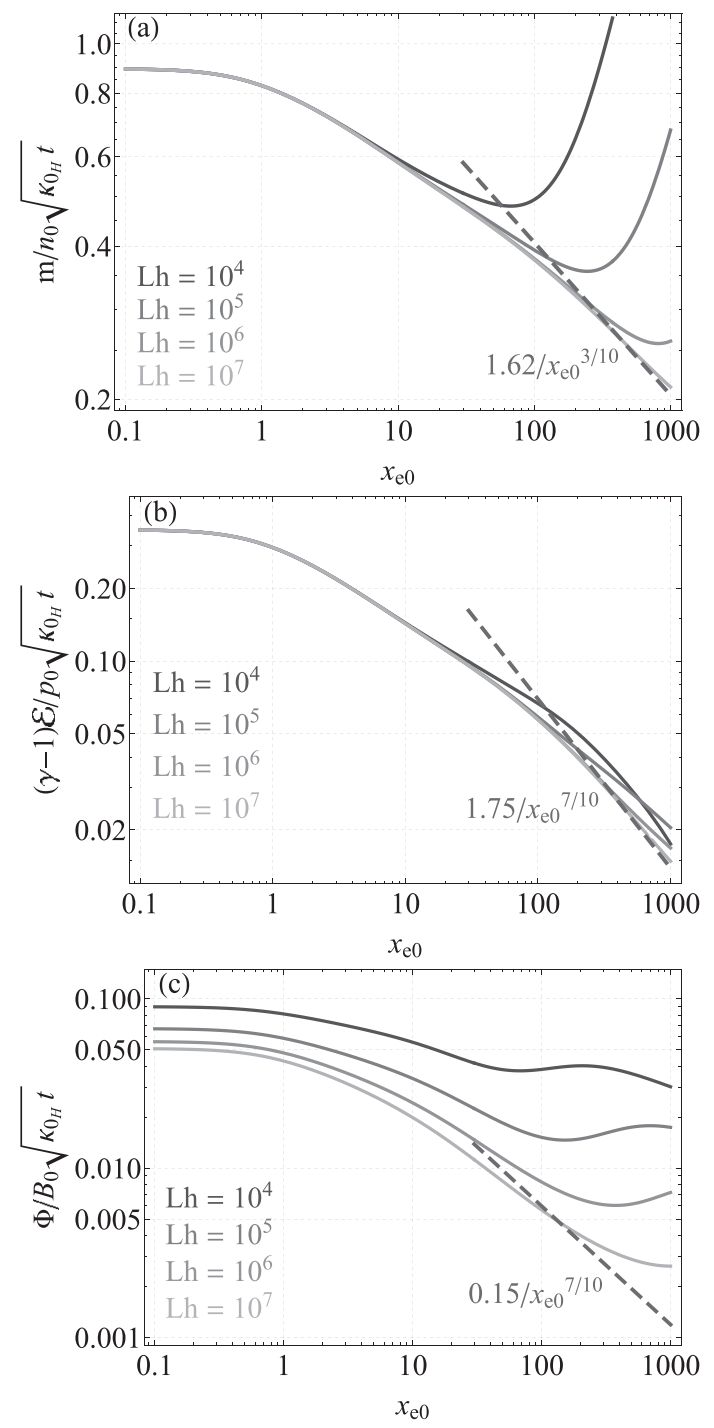

FIG. 5. Normalized ablated mass $m / n_{0} \sqrt{\kappa_{0_{H}} t}$, thermal energy losses $(\gamma-1) \mathcal{E} / p_{0} \sqrt{\kappa_{0 H} t}$, and magnetic flux losses $\Phi / B_{0} \sqrt{\kappa_{0_{H}} t}$ vs. $x_{e 0}$ keeping Lh fixed. The dashed lines correspond to the scaling laws obtained in an infinite $\beta$ model in Ref. 10.

\section{AN APPROXIMATE MODEL}

Defining

$$
\zeta=\frac{2-\gamma}{\gamma(\beta+1)},
$$

the energy equation (13) gets simplified if we assume $\zeta \ll 1$, which takes place for large $\beta$ or $\gamma$ close to 2 . In our case, $\gamma=5 / 3$, and $\zeta$ can take the value $\zeta=1 / 5$ at most. In this limit, the energy equation can be integrated once since the last term in the right hand side vanishes, yielding

$$
\begin{aligned}
V(\eta)= & 2 \mathcal{P}_{c} \theta^{5 / 2} \frac{\mathrm{d} \theta}{\mathrm{d} \eta}+2 \frac{\mathcal{P}_{n} \mathcal{P}_{c}}{\beta+1} \frac{\theta^{5 / 2} \phi}{1-\frac{\phi^{2}}{\beta+1}} \frac{\mathrm{d}(\phi \theta)}{\mathrm{d} \eta} \\
& +\frac{2}{\operatorname{Le}(\beta+1)} \frac{\mathcal{P}_{d}}{\theta^{3 / 2}} \phi \frac{\mathrm{d} \phi}{\mathrm{d} \eta} .
\end{aligned}
$$

Notice that the velocity at infinite $V_{\infty}$ vanishes in this limit. Inserting this expression into the induction equation (14) yields

$$
\begin{aligned}
-\eta & \frac{\mathrm{d} \phi}{\mathrm{d} \eta}+\frac{\mathrm{d}}{\mathrm{d} \eta}\left[2 \mathcal{P}_{c}\left(1-\mathcal{P}_{n}\right) \theta^{5 / 2} \frac{\mathrm{d} \theta}{\mathrm{d} \eta} \phi\right] \\
= & \frac{2}{\mathrm{Le}} \frac{\mathrm{d}}{\mathrm{d} \eta}\left[\frac{\mathcal{P}_{d}\left(1-\mathcal{P}_{e}\right)}{\theta^{3 / 2}}\left(1-\frac{\phi^{2}}{\beta+1}\right) \frac{\mathrm{d} \phi}{\mathrm{d} \eta}\right] .
\end{aligned}
$$

It can be seen that magnetic field convection due to the Ettingshausen and Joule contributions to velocity has a diffusion structure with negative coefficient that relieves the original diffusivity. The term

$$
\mathcal{P}_{e}\left(x_{e}\right)=\frac{(\gamma-1) \gamma_{0}}{8 \gamma \alpha_{0}} \frac{x_{e}^{2} \mathcal{P}_{c} \mathcal{P}_{n}}{\mathcal{P}_{d}}
$$

comes from the Ettingshausen contribution, which can reduce it by a factor of 0.36 when the plasma is highly magnetized [see Fig. 2(b)]. The coefficient $\left[1-\phi^{2} /(\beta+1)\right]$ is due to the Joule contribution. It can take small values for $\beta \ll 1$ and $\phi \approx 1$, making the magnetic field profile present a sharp corner (a discontinuity in the first derivative), as shown in Fig. 4.

The governing equations in a small $\zeta$ limit would therefore be reduced to Eq. (32) together with Eq. (12), which, with the use of the equation of state (7), stands for a system of two equations of fourth order for $\theta$ and $\phi$.

In Fig. 6, mass ablation and magnetic flux losses computed using the exact model [Eqs. (12)-(14)] and the model taking the $\zeta \ll 1$ limit [Eqs. (12) and (32)] are shown for increasing $x_{e 0}$ keeping a fixed $\beta=1$. It can be seen that the approximation $\zeta \ll 1$ is reasonably good. It underestimates magnetic flux losses and overestimates mass ablation with less than $10 \%$ difference.

\section{CONCLUSIONS}

Magnetic pressure effects in a relevant MagLIF fusionlike plasma have been investigated by extending the analysis performed in Ref. 10 to an arbitrary pressure ratio $\beta$. The solution preserves the self-similar nature and depends on two

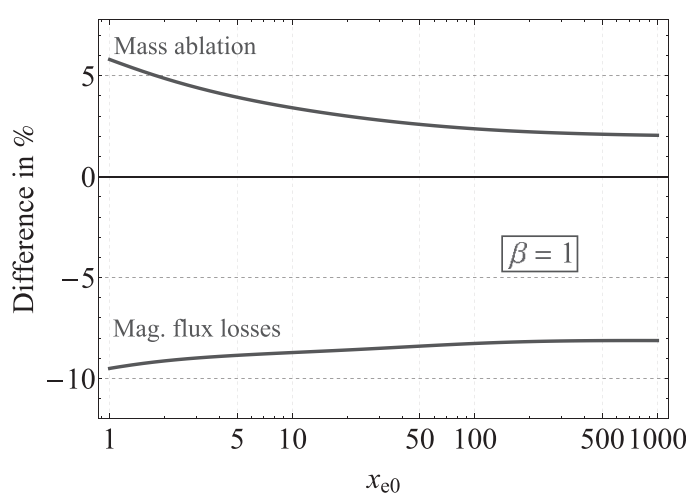

FIG. 6. Mass ablation and magnetic flux losses difference between the exact model and the $\zeta \ll 1$ approximation. The difference is computed as $100 \times\left(\left.m\right|_{\text {Approx }}-\left.m\right|_{\text {Exact }}\right) /\left.m\right|_{\text {Exact }}$ and $100 \times\left(\left.\Phi\right|_{\text {Approx }}-\left.\Phi\right|_{\text {Exact }}\right) /\left.\Phi\right|_{\text {Exact }}$, respectively. 
free parameters: the initial electron Hall parameter $x_{e 0}$ and the magnetic Lewis number Le, being the pressure ratio $\beta$ determined by Eq. (9).

Nernst, Ettingshausen, and Joule effects come into play together with thermal conduction in the energy balance equation. Due to the presence of the cold unmagnetized liner, the region of the hot spot adjacent to it is governed by the thermal conduction. Far from the liner, however, all effects are of the same order of magnitude when $\beta$ is of order unity, while Joule dissipation becomes the most important effect for small $\beta$.

The parameter $\beta$ measures the ratio between the thermal and magnetic diffusion scale lengths. When it is large, magnetic field is diffused in thin layers compared to the characteristic length of the thermal problem. When it is of order unity, magnetic field diffusion and thermal conduction take place in the same scale length. When it is small, magnetic diffusion becomes predominant, inducing temperature changes due to Joule heating. In this limit, the scale length of the problem scales as $\epsilon \sim 1 / \sqrt{\mathrm{Le}}$, and increasing the magnetization further does not affect the temperature and magnetic field profiles appreciably.

Magnetization of the hot spot is intended to reduce thermal energy and magnetic flux losses. They are shown to decrease when the plasma gets magnetized until $\beta$ values of order unity are reached. From this point forward, magnetic flux conservation is no longer improved when the magnetization is further increased, while magnetic pressure gradients enhance mass ablation notoriously.

The system of governing equations is greatly simplified while still retaining the main finite $\beta$ terms if we assume $(2-\gamma) / \gamma(\beta+1) \ll 1$. The equation of energy can be integrated once, yielding an explicit expression for plasma velocity. The error introduced by this approximation is shown to be relatively small when compared to the exact model. This limit is interesting as a straightforward way to include magnetic pressure effects in numerical codes or in theoretical models to study the evolution of the hot spot of magnetized plasma implosions. ${ }^{11}$

\section{ACKNOWLEDGMENTS}

This research was supported by the Spanish Ministerio de Economía y Competitividad, Project No. ENE201454960R, and by the Spanish Ministerio de Educación, Cultura y Deporte under the National Research Program FPU, Grant No. FPU 14/04879.

${ }^{1}$ S. A. Slutz, M. C. Herrmann, R. A. Vesey, A. B. Sefkow, D. B. Sinars, D. C. Rovang, K. J. Peterson, and M. E. Cuneo, Phys. Plasmas 17, 056303 (2010).

${ }^{2}$ R. Landshoff, Phys. Rev. 76, 904 (1949).

${ }^{3}$ M. M. Basko, A. J. Kemp, and J. Meyer-ter Vehn, Nucl. Fusion 40, 59 (2000).

${ }^{4}$ S. A. Slutz and R. A. Vesey, Phys. Rev. Lett. 108, 025003 (2012).

${ }^{5}$ S. A. Slutz, W. A. Stygar, M. R. Gomez, K. J. Peterson, A. B. Sefkow, D. B. Sinars, R. A. Vesey, E. M. Campbell, and R. Betti, Phys. Plasmas 23, 022702 (2016)

${ }^{6}$ M. R. Gomez, S. A. Slutz, A. B. Sefkow, D. B. Sinars, K. D. Hahn, S. B. Hansen, E. C. Harding, P. F. Knapp, P. F. Schmit, C. A. Jennings et al., Phys. Rev. Lett. 113, 155003 (2014)

${ }^{7}$ J. R. Davies, D. H. Barnak, R. Betti, E. M. Campbell, P.-Y. Chang, A. B. Sefkow, K. J. Peterson, D. B. Sinars, and M. R. Weis, Phys. Plasmas 24, 062701 (2017).

${ }^{8}$ D. H. Barnak, J. R. Davies, R. Betti, M. J. Bonino, E. M. Campbell, V. Y. Glebov, D. R. Harding, J. P. Knauer, S. P. Regan, A. B. Sefkow et al., Phys. Plasmas 24, 056310 (2017).

${ }^{9}$ A. L. Velikovich, J. L. Giuliani, and S. T. Zalesak, Phys. Plasmas 22, 042702 (2015)

${ }^{10}$ F. García-Rubio and J. Sanz, Phys. Plasmas 24, 072710 (2017).

${ }^{11}$ F. García-Rubio, J. Sanz, and R. Betti, Phys. Rev. E 97, 011201(R) (2018).

${ }^{12}$ S. Fujioka, Z. Zhang, K. Ishihara, K. Shigemori, Y. Hironaka, T. Johzaki, A. Sunahara, N. Yamamoto, H. Nakashima, T. Watanabe, H. Shiraga, H. Nishimura, and H. Azechi, Sci. Rep. 3, 1170 (2013).

${ }^{13}$ V. T. Tikhonchuk, M. Bailly-Grandvaux, J. J. Santos, and A. Poyé, Phys. Rev. E 96, 023202 (2017).

${ }^{14}$ S. F. Garanin, J. Appl. Mech. Tech. Phys. 28, 816-819 (1987).

${ }^{15}$ S. F. Garanin and V. I. Mamyshev, J. Appl. Mech. Tech. Phys. 31, 28-34 (1990).

${ }^{16}$ S. I. Braginskii, in Reviews of Plasma Physics, edited by M. A. Leontovich (Consultants Bureau, New York, 1965).

${ }^{17}$ R. Betti, M. Umansky, V. Lobatchev, V. N. Goncharov, and R. L. McCrory, Phys. Plasmas 8, 5257-5267 (2001).

${ }^{18}$ J. Sanz and R. Betti, Phys. Plasmas 12, 042704 (2005).

${ }^{19}$ Y. B. Zel'dovich and Y. P. Raizer, Physics of Shock Waves and HighTemperature Hydrodynamic Phenomena (Dover Publication, 2002). 



\section{Plasma-liner interface: mass diffusion and liner material effects}

\subsection{General overview}

In this article, a theoretical analysis of liner-fuel mass diffusion and the effect of the liner material on mass ablation, energy and magnetic flux losses in a magnetized plasma implosion context is performed. For this purpose, the study presented in Chapter 3 has been extended to liner materials of an arbitrary atomic number.

The motivation to carry out this study comes from the versatility on the choice of the liner material in magneto inertial fusion (MIF) schemes. In the original magnetized liner inertial fusion (MagLIF) concept, the liner is made of a conductive material [84]. MagLIF implosions are driven by a pulsed-power machine (Sandia's $\mathrm{Z}$ facility [69]), and typically, low atomic number metals such as lithium, beryllium or aluminum are used. In laser-driven MagLIF implosions performed at OMEGA, the liner does not need to be conductive. It is made of plastic, less dense, cheaper and safer to handle than low atomic number metals $[26,8]$.

In both pulsed-power-driven and laser-driven MagLIF schemes, it is expected that the inner side of the liner gets heated and ablates into the hot spot, according to the same physical mechanism that leads to shell ablation in inertial confinement fusion (ICF) capsules [18, 13, 73, 74]. The choice of the liner material would therefore affect the thermal energy losses in the fuel, and the magnetic flux conservation in the hot spot. Additionally, at the interface between the ablated liner material and the fuel, mass mixing due to molecular diffusion processes can take place. The fuel gets thereby polluted and the quality of the implosion may be affected.

Diffusion of ion species in multi-component plasmas has recently gained attention in ICF as a potential mechanism leading to the observed yield anomalies [3, 4, 21]. They are conjectured to be caused by stratification of the fuel in the implosion core arisen from the shock-driven diffusion [3, 11, 10]. However, liner-fuel diffusion mixing in a MagLIF context has not been given the same attention and its effect on the implosion performance still remains an open question.

Mass diffusion in plasmas can be driven by gradients of species concentration, pressure, electrostatic potential as well as ion and electron temperatures. The latter three give rise to baro-diffusion, electro-diffusion and thermo-diffusion, respectively. 
The derivation of every diffusion coefficient has been subject of intense recent research. Kagan and Tang [48] extended the thermodynamic technique of Landau and Lifshitz [54], originally developed for inter-species diffusion in a binary neutral gas mixture, to a quasi-neutral plasma with two ion species. They proved that baro- and electro-diffusion ratios $k_{p}, k_{e}$ are a thermodynamic quantity and can be obtained without invoking a collisional model. The baro-diffusion ratio $k_{p}$ results to be identical to its counterpart in a neutral gas mix, and the electro-diffusion ratio $k_{e}$ shows a strong dependence on the charge-to-mass ratio of the ion species [47]. Thermodiffusion arises from the thermal force in the friction drag between electrons and ions and between ion species [49]. The thermo-diffusion ratio $k_{T}$ depends then on the nature of the collisions between particles. To compute such ratio, the authors in Ref. [49] followed the monograph by Zhdanov [94], and employed the first 21 moments of the kinetic equation for each species's distribution function. Due to the long-range nature of the Coulomb potential, plasma thermo-diffusion plays a more important role in plasmas compared to the neutral gas mixtures.

It is not until the recent work of Simakov, Molvig and collaborators [80, 63, 81, 82] that the fluid equations for multi-component plasmas are closed with the expressions derived for individual ion species drift velocities, total ion heat flux and viscosity. The authors solved the kinetic equations with a generalized Chapman-Enskog expansion that assumes small Knudsen numbers for all species. In Ref. [63], the electron and ion transport theories are developed for a two ion species plasma with disparate masses. In this reference, the transport problem is written as a two dimensional Onsager matrix, following the formulation and notation of Hazeltine and Waelbroeck [42] and Helander and Sigmar [43]. In Refs. [81, 82], the analysis is extended to plasmas with multiple ion species of arbitrary mass by performing a generalization of the Braginskii ion fluid description [20].

The theory of multi-component plasmas has been applied in Ref. [64] to describe the theoretical structure of the plasma diffusion layer that develops from an initially sharp gas-metal interface. Isothermal and isobaric conditions are assumed, so only mass diffusion process takes place. The solution is self-similar, and the structure of the layer presents highly nonlinear features with a sharp front defined on the gas side. As explained in this chapter, a similar structure develops at the interface between the ablated liner and the fuel analyzed here.

\subsection{Statement of the problem}

In this article, a similar problem to the one analyzed in Chapter 3 is considered. The evolution of a semi-infinite slab of hot magnetized plasma (fuel) in contact with a cold dense unmagnetized plasma (liner), as sketched in Fig. 1 in the article, is studied. The motion is one-dimensional, and the only independent variables are the streamwise coordinate $x$ and time $t$. Both plasmas are fully ionized, with $Z$ denoting the atomic number. The fuel is made of deuterium, $Z=Z_{1}=1$, but the 
liner material is left as a free parameter, arbitrary $Z=Z_{2}$, which is the novelty introduced in this article.

The qualitative description of the motion goes as follows. The fuel is initially in contact with the liner at $x=0$. Due to thermal conduction, the liner ablates and compresses the fuel. The problem is considered to be subsonic (small Mach number), and the thermal-to-magnetic pressure ratio $\beta$ is high, which leads to isobaricity. The surface $x=0$ represents therefore an ablation front. Another surface, $x=x_{b}(t)>0$, called ablated border, stands for the interface between the ablated liner material and the fuel. The region $x>0$, which would crudely represent the hot spot, is therefore formed of both liner and fuel materials. The transport coefficients depend on the atomic number $Z$, and consequently they change along the streamwise direction $x$.

In the first part of the paper, the liner and fuel materials are considered to be immiscible. The atomic number takes the value $Z=Z_{2}$ for $x<x_{b}$ and $Z=Z_{1}$ for $x>x_{b}$. The difference in atomic number leads to an imbalance in the electron pressure, which has to be counterbalanced by a plasma density jump in order to restore isobaricity. The ablated border stands then for a contact discontinuity through which temperature, magnetic field and ion velocity are continuous. However, since the thermal conductivity changes with $Z$, the temperature gradient is discontinuous to equalize the heat flux at both sides of the ablated border. The magnetic field is convected out of the fuel by the Nernst term. The Nernst convection velocity depends on $Z$, and attains a higher value at the liner side. In order to equalize the amount of magnetic field convected at both sides of the ablated border, it is dissipated in a thin layer on the fuel side, and its value is reduced. Then, it penetrates into the ablated liner material, and is convected towards the liner where it is totally dissipated in second diffusive boundary layer.

In the second part of the paper, the same problem is studied taking into account liner-fuel mixing due to mass diffusion. Only the unmagnetized plasma limit is investigated and the theory developed in Refs. [81, 82] for multi-component unmagnetized plasmas is applied for the transport terms. The ablated liner material ablates and compresses the fuel. This process is governed by heat conduction. Simultaneously, liner and fuel are mixed at the ablated border, this process being governed by mass diffusion. The ratio between heat and mass diffusivities, $\kappa_{0}$ and $\nu_{0}$, respectively, corresponds to the Lewis number Le $\equiv \kappa_{0} / \nu_{0}$. It only depends on the liner and fuel material properties, and is shown to be large in MagLIF applications. This implies that mass diffusion is confined within a thin layer compared to the characteristic length of the ablative wave.

\subsubsection{Methodology}

Plasma motion and magnetic field evolution in the hot spot are governed by the ion continuity, total momentum and total energy conservation equations together with Faraday's law (induction equation) and the equation of state, Eqs. (1) - (5) in the 
article. The total momentum equation reduces to isobaricity. The energy conservation equation can be integrated, yielding an explicit expression for ion velocity, Eq. (6), which turns out to be proportional to the heat flux. The system of equations can therefore be reduced to ion continuity and induction: two equations for the two unknowns temperature $T$ and magnetic field $B$. The position of the ablated border, $x_{b}(t)$, has to be self-consistently determined by applying the fluid surface condition, Eq. (7). As boundary conditions, it is imposed that the plasma recovers the initial fuel state $T=T_{0}, B=B_{0}$ at $x \rightarrow \infty$, and $T / T_{0} \rightarrow 0, B / B_{0} \rightarrow 0$ at $x=0$.

The solution is sought under the form of a self-similar diffusive wave [93]. The selfsimilar coordinate $\eta=x / \sqrt{\kappa_{0} t}$ is introduced, with $\kappa_{0}$ being the thermal diffusivity in the unperturbed fuel. Ion velocity is consistently scaled as $V=2 \sqrt{t / \kappa_{0}} v$. Temperature and magnetic field are normalized with their initial value on the fuel: $\theta=T / T_{0}$, $\phi=B / B_{0}$. The dimensionless governing equations become then Eqs. (23) and (24) in the article. They stand for a system of ordinary differential equations of fourth order. The aforementioned boundary conditions become $\theta=\phi=0$ at $\eta=0$ and $\theta=\phi=1$ at $\eta \rightarrow \infty$. At the ablated border $\eta=\eta_{b}$, temperature and magnetic field derivatives are discontinuous and given by Eqs. (30) and (31). It can be seen that the position of the ablated border needs to be determined self-consistently with $\theta$ and $\phi$. It cannot be determined a posteriori as was the case in Chapters 3 and 4 .

The system of equations depends on three free parameters: the magnetic Lewis number $\mathrm{Le}_{\mathrm{m}}$, defined as the ratio between thermal and magnetic diffusivities in the unperturbed fuel, as likewise done in Chapters 3 and 4, the electron Hall parameter of the unperturbed fuel $x_{e 0}$ and the atomic number of the liner $Z_{2}$. The atomic number comes into play in the transport terms and in the electron Hall parameter anywhere in the hot spot $x_{e}$, given by Eq. (13).

This boundary problem has been numerically computed designing a shooting method. An asymptotic analysis of the governing equations for $\eta \ll 1$ shows that the temperature and magnetic field profiles close to the ablation front take the form $\left.\theta\right|_{\eta \ll 1}=$ $s_{\theta} \eta^{2 / 5},\left.\phi\right|_{\eta \ll 1}=\operatorname{Le}_{\mathrm{m}} s_{\phi} \eta^{8 / 5}$. The parameters $s_{\theta}\left(\mathrm{Le}_{\mathrm{m}}, x_{e 0}, Z_{2}\right), s_{\phi}\left(\mathrm{Le}_{\mathrm{m}}, x_{e 0}, Z_{2}\right)$ are positive and obtained by solving the complete problem with the boundary conditions far from the liner. The shooting method goes as follows. First, an arbitrary value is given to $s_{\theta}$ and $s_{\phi}$. Second, the equations are integrated starting form he ablation front $\eta \ll 1$ and taking the liner atomic number, $Z=Z_{2}$, until a sufficiently large $\eta=\eta^{*}$. Third, the velocity is calculated as given by Eq. (20), and the ablated border position is computed from the fluid surface condition $V\left(\eta_{b}\right)=\eta_{b}$. Fourth, the jump conditions at the ablated border are applied, Eqs. (30) and (31), and the equations are further integrated taking the fuel atomic number, $Z=Z_{1}$ from $\eta=\eta_{b}$ until $\eta=\eta^{*}$. The parameters $s_{\theta}$ and $s_{\phi}$ have been obtained by imposing $\theta\left(\eta^{*}\right)=1$, $\phi\left(\eta^{*}\right)=1$. It has been found that $\eta^{*}=8$ is sufficiently large to yield accurate results.

Apart from the numerical computation of the equations, an analytical solution has been obtained in the limit of large $Z_{2}$. Asymptotic analysis and singular perturbation 
theory has been extensively applied to obtain the structure of the layers composing the solution, shown in detail in Sec. III B in the article.

Integral quantities such as mass ablation $m$ and magnetic flux losses $\Phi$ per unit area in the hot spot, and thermal energy losses $\mathcal{E}$ and magnetic flux losses $\tilde{\Phi}$ per unit area in the fuel have been computed, showing the effect of the liner atomic number $Z_{2}$.

When the problem is reformulated taking into account mass diffusion, only the unmagnetized limit is studied, and the evolution of the magnetic field has been disregarded. Additionally to total ion continuity, total momentum and energy conservation equations, the fuel continuity equation has to be self-consistently solved, Eq. (63). The system can be reduced to total ion continuity and fuel mass conservation: two equations for the unknowns temperature $T$ and fuel mass concentration $y$. The expressions for electron and ion heat fluxes and fuel drift velocity, taken from Refs. [81, 82], are given in Eqs. (A7), (A18) and (A16) in the Appendix of the article, respectively.

The solution still preserves the self-similar diffusive wave structure when mass diffusion comes into play. A similar normalization is consequently introduced, and the system of equations is reduced to Eqs. (68) and (69) in the article, with the fuel drift velocity given by Eq. (72). It consists of a system of differential equations of fourth order. It is completed with the boundary conditions $\theta=y=0$ at $\eta=0$ and $\theta=y=1$ at $\eta \rightarrow \infty$. The Lewis number naturally appears in the fuel drift velocity. The only free parameter governing the system is the liner atomic number $Z_{2}$.

An asymptotic analysis of the equations in the large $Z_{2}$ limit has been performed to obtain the structure and the scaling of the width of the mass diffusion layer, as shown in Sec. V A in the article.

The numerical computation of the system has significant differences with respect to the problem without mass diffusion. This comes from the fact that the fuel concentration $y$ decreases exponentially when one moves from $\eta=\eta_{b}$ into the ablated liner material. The shape of the fuel concentration profile close to the ablation front is very flat and close to zero, $y \sim \eta^{\mathrm{Le}}$, which cannot be properly captured numerically. Therefore, the following strategy has been designed. It is assumed an initial fuel concentration based on the scalings derived for large $Z_{2}$ : $\left.y\right|_{\text {init }}=\left\{1+\tanh \left[\left(\eta-\eta_{b 0}\right) \sqrt{\mathrm{Le}}\right]\right\} / 2$, with $\eta_{b 0}$ corresponding to an initial guess of the ablated border position, taken as its solution for $Z_{2}=1, \eta_{b 0}=0.35$. With this fuel concentration profile, the ion continuity, Eq. (68), is computed to obtain the temperature profile $\theta$. In this computation, a shooting method has been used assuming a temperature profile close to the liner $\left.\theta\right|_{\eta \ll 1}=s_{\theta} \eta^{2 / 5}$, and integrating from the ablation front towards the hot spot. The parameter $s_{\theta}$ is obtained imposing $\theta\left(\eta^{*}\right)=1$, where $\eta^{*}=8$ is sufficiently large to yield accurate results. The ion velocity profile and the ablated border position can be subsequently obtained. The fuel mass continuity, Eq. (69), is therefore computed with the given $\theta, V$ and $\eta_{b}$. A double shooting method was found to be optimum to solve this equation. 
An initial guess for the value and derivative of $y$ at the ablated border is proposed, $y\left(\eta_{b}\right)=s_{y}$ and $y^{\prime}\left(\eta_{b}\right)=\sqrt{\mathrm{Le}} s_{y p}$. The fuel concentration equation is integrated from $\eta=\eta_{b}$ both towards the liner and towards the fuel. The parameters $s_{y}$ and $s_{y p}$ are hence obtained imposing $y(0)=0, y\left(\eta^{*}\right)=1$. Once the fuel concentration is obtained, temperature is recomputed again from the ion continuity and the process is repeated until the profiles converge. The convergence of the method is very fast, and five iterations were enough to yield accurate results.

Finally, the amount of liner material diffused into the fuel per unit area, $m_{l f}$ defined in Eq. (88), has been computed and compared to the total mass of the liner ablated $m$.

\subsection{Results}

First, the results without mass diffusion are discussed. Temperature and magnetic field profiles in the unmagnetized case are shown in Fig. 3(a) in the article, and two liner material cases are compared: deuterium $\left(Z_{2}=1\right)$ and beryllium $\left(Z_{2}=4\right)$. It can be seen that the plasma temperature in the hot spot is slightly higher in the second case. However, the most striking difference lies in the magnetic field profile. When the liner is made of beryllium, the magnetic field experiences an abrupt decrease in a thin layer at the fuel side of the ablated border. As discussed in Sec. 5.2, this decrease comes from the Nernst convection velocity dependence on $Z$. An asymptotic analysis has been performed to elucidate the structure of this layer, which shows that its width scales as $O\left(1 / \mathrm{Le}_{\mathrm{m}}\right)$. It is thinner than the second diffusive layer taking place at the ablation front, which scales as $O\left(1 / \sqrt{\mathrm{Le}_{\mathrm{m}}}\right)$.

In Fig. 3(b), mass density profile is plotted for a beryllium liner material. It presents a discontinuity at the ablated border, attaining a lower value on the fuel side. The ablated border stands therefore for a surface discontinuity moving with negative acceleration $g=\mathrm{d}^{2} x_{b} / \mathrm{d} t^{2}=-\eta_{b} \sqrt{\kappa_{0}} / 4 t^{2}<0$, where a light fluid (fuel) is pushing backwards a heavier fluid (liner material). This hydrodynamic structure is susceptible to be Rayleigh-Taylor unstable.

The asymptotic analysis performed in the large $Z_{2}$ limit yields the following result. Both thermal and electrical conductivities decrease with $Z$. The motion of the plasma, governed by heat flux, is strongly suppressed. The ablated material penetrates less into the hot spot, and the position of the ablated bored steps back and scales as $O\left(1 / \sqrt{Z_{2}}\right)$. The effective magnetic Lewis number in high- $Z$ ablated region turns out to be smaller than the characteristic one in the fuel: $\mathrm{Le}_{l} \sim \mathrm{Le}_{\mathrm{m}} / Z_{2}^{2}$. The diffusive layer close to the ablation front is therefore wider since its width scales as $O\left(Z_{2} / \sqrt{\mathrm{Le}_{\mathrm{m}}}\right)$. The four integral quantities $m, \Phi, \mathcal{E}$ and $\tilde{\Phi}$ are shown to decrease as $1 / \sqrt{Z_{2}}$. The ratio $\Phi / \tilde{\Phi}$ attains a constant value $\Phi / \tilde{\Phi}=0.36$, which implies that $64 \%$ of the magnetic flux losses in the fuel are spent into magnetizing the ablated material, while the remaining $36 \%$ is lost through dissipation at the liner. 
In Fig. 4, the four integral quantities are computed as a function of the liner atomic number and compared to the scaling laws derived in the large $Z_{2}$ limit. Both thermal energy and magnetic flux losses in the fuel decrease monotonically with $Z_{2}$ due to heat flux reduction. However, mass ablation and magnetic flux losses in the hot spot attain a maximum at $Z_{2}=4$ and $Z_{2}=12$, respectively.

Next, the results with mass ablation are discussed. Temperature and velocity profiles are shown in Fig. 8(a) for a beryllium liner material. They are compared to the solution of the equations disregarding mass diffusion, being almost identical. This suggests that, in a MagLIF context, the dynamics of the hot spot can be computed independently of the mass transport problem, and the latter can be solved a posteriori. Fuel concentration profiles for different liner materials are plotted in Fig. 8(c). It can be seen that the mixing layer shrinks when the atomic number increases.

Mass transport takes place in a thin layer developing at the ablated border. An asymptotic analysis expanding the governing equations around $\eta=\eta_{b}$ has been performed in order to obtain the structure of this layer, as explained in Sec. V A in the article. The width of the layer scales as $O(1 / \sqrt{\mathrm{Le}})$. Due to the small variations of temperature in the scale length of the layer, the latter develops as if the problem was isothermal. Concentration gradients and baro-diffusion become the main mechanisms governing mass transport, and thermo-diffusion plays a secondary role. The boundary layer shares therefore similarities with the problem analyzed in Ref. [64]. Particularly, as can be seen in Fig. 9, it presents a well-defined wave front on the fuel side after which the fuel remains clean. The fuel concentration profile obtained by this boundary layer model is compared to the solution of the whole system in Fig. 8(c) for an aluminum liner, showing good agreement. This fact reinforces the aforementioned suggestion that mass transport can be computed once the hot spot dynamics are solved.

The amount of liner material diffused into the fuel per unit area, $m_{l f}$, has been computed and compared to the liner mass ablation $m$. As shown in Table I in the article, around $4 \%$ of the ablated mass is diffused into the fuel. This percentage slightly decreases with $Z_{2}$, but attains the asymptotic limit $100 \times m_{l f} / m=2.36 \%$ and cannot get further reduced. Finally, the width of the mixing layer due to mass diffusion has been compared to the width of the turbulent mixing layer that can develop from the Rayleigh-Taylor instability that can take place at the ablated border. The ratio, shown in Table I, is of order unity. This indicates that liner fuel mixing by microscopic motion (diffusion) may therefore be as important as mixing due to macroscopic motion (hydrodynamic instabilities leading to turbulence), which justifies the importance of studying mass transport in magnetized implosions. 



\title{
Mass diffusion and liner material effect in a MagLIF fusion-like plasma
}

\author{
F. García-Rubio a) and J. Sanz \\ E.T.S.I. Aeronáutica y del Espacio, Universidad Politécnica de Madrid, Madrid 28040, Spain
}

(Received 14 June 2018; accepted 30 July 2018; published online 16 August 2018)

\begin{abstract}
In this paper, liner-fuel mass diffusion and the effect of the liner material on mass ablation, energy, and magnetic flux losses are studied in a MagLIF fusion-like plasma. The analysis performed in [García-Rubio and Sanz, Phys. Plasmas 24, 072710 (2017)] is extended to liner materials of an arbitrary atomic number. The liner ablates and penetrates into the hot spot, compressing and cooling down the fuel. The magnetic flux in the fuel is lost by Nernst convection through the ablated liner-fuel interface, called ablated border. Increasing the liner atomic number leads to a reduction of both energy and magnetic flux losses in the fuel for small and moderate magnetization values. Mass diffusion is confined within a thin layer at the ablated border. Concentration gradient and baro-diffusion are the predominant mechanisms leading to liner-fuel mixing. The width of the diffusion layer may be comparable to the turbulent mixing layer resulting from the Rayleigh-Taylor instability at the ablated border. An asymptotic analysis performed for large liner atomic number $Z_{2}$ shows that mass ablation, energy and magnetic flux losses, and liner-fuel mass diffusion scale as $1 / \sqrt{Z_{2}}$. Published by AIP Publishing. https://doi.org/10.1063/1.5044642
\end{abstract}

\section{INTRODUCTION}

In the recently proposed magnetized liner inertial fusion (MagLIF) scheme, a pulsed power machine drives the implosion of a conductive cylindrical liner filled fuel that is magnetized and preheated. ${ }^{1,2}$ The advantages of magnetizing the fuel lie both on reducing heat $\operatorname{losses}^{3}$ and enhancing $\alpha$-particles energy deposition. ${ }^{4}$ The liner is typically made of low atomic number metals such as lithium, beryllium, or aluminum. MagLIF concept has been scaled down in size to OMEGA laser facility, ${ }^{5,6}$ where a laser drives the implosion of a parylene-Z plastic liner, less dense than metal liners.

A relevant feature of magnetized implosions is the long time that the hot spot and the cold liner stay in contact. Understanding the effect of fuel magnetization on heat and magnetic flux losses through the hot spot-liner interface becomes essential. For this purpose, the evolution of a hot magnetized plasma (hot spot) in contact with a cold unmagnetized liner has recently been investigated in planar geometry. ${ }^{7,8}$ The problem is studied in the low Mach number and high thermal to magnetic pressure ratio $\beta$ limit, which implies isobaricity. In Ref. 8, the liner is treated as a cold dense plasma made of the same material as the fuel (deuterium), aiming to model the cryogenic fuel layer added on the inner side of the liner in high-gain MagLIF configurations. ${ }^{9}$ The hot spot-liner interface represents an ablation front through which the liner ablates, penetrates into the hot spot, and cools it down by thermal conduction. The interface separating the ablated liner material and the fuel is referred to as ablated border. In Ref. 10, this study was revisited, including finite pressure ratio $\beta$ effects.

In this paper, we extend the analysis performed in Ref. 8 to liners made of an arbitrary material (see Fig. 1). The plasma ablated into the hot spot has therefore a different

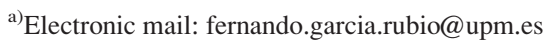

atomic number $Z$ compared to the fuel. In the first part of this paper, the two plasmas are treated as immiscible, and the ablated border appears as a contact discontinuity where certain jump conditions must be satisfied. In the second part of this paper, we let the two plasmas diffuse and analyze liner and fuel mixing.

Diffusion of ion species in multi-component plasmas has recently gained attention as a potential mechanism explaining the observed yield anomalies in inertial confinement fusion (ICF) implosions. ${ }^{11,12}$ In plasmas, such diffusion can be driven by concentration, pressure, electrostatic potential, and temperature gradients. The latter three are defined as baro-diffusion, electro-diffusion, and thermo-diffusion, respectively. Kagan and Tang ${ }^{13,14}$ obtained the baro- and electro-diffusion ratios $k_{p}, k_{e}$ without invoking a collisional model, proving that both ratios are a thermodynamic quantity. In the authors' words, baro- and electro-diffusion are "inextricably intertwined," and while $k_{p}$ and $k_{e}$ depend on the choice of the thermodynamic system (including ions only or the plasma as a whole), the overall diffusive flux stays the same regardless of this choice. ${ }^{14}$ When the thermodynamic system includes the ions only, the baro-diffusion ratio $k_{p}$ turns out to be identical to its counterpart in a neutral gas mix ${ }^{15}$ and the electro-diffusion ratio $k_{e}$ depends on the charge-to-mass ratio of the ion species. ${ }^{13}$ Thermo-diffusion, on its part, arises from the thermal force in the friction drag between different ion species and between electrons and ions. It depends on the nature of the collisions between particles. Due to the long range of the Coulomb collisions, it plays a more important role in plasmas compared to neutral gas mixtures. In addition, ion thermo-diffusion reinforces baro-diffusion in plasmas, in contrast to the neutral mixture case. $^{16}$

In the work done by Simakov, Molvig, and collaborators, ${ }^{17-20}$ the kinetic equations of multi-component plasmas are solved by a generalized Chapman-Enskog expansion that 


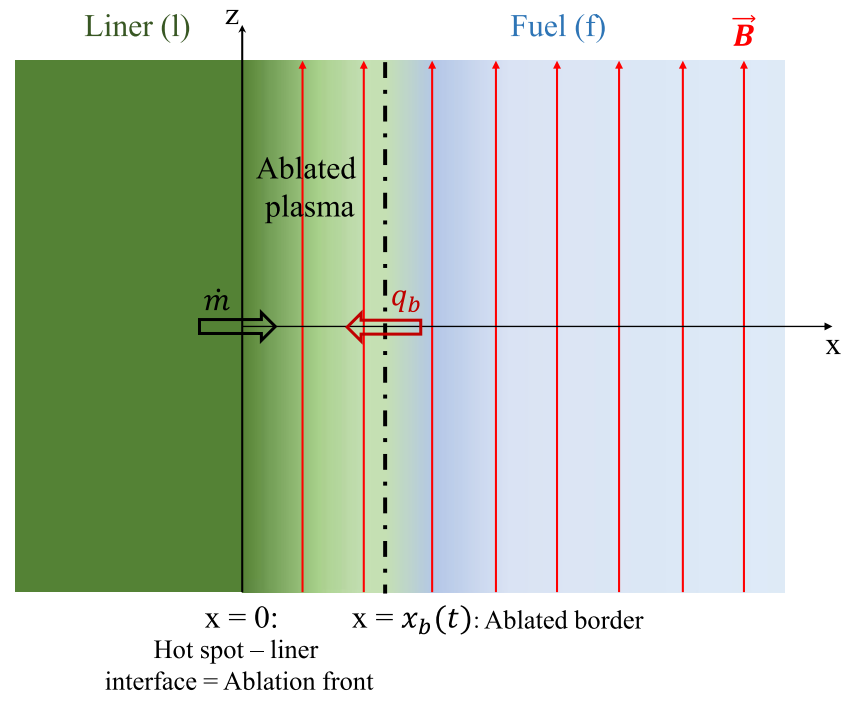

FIG. 1. Artistic scheme of the problem presented. The color gradation represents ion density. Liner material (l) is shown in green, while fuel material (f) is shown in blue. Arrows in red stand for magnetic field lines.

assumes small Knudsen numbers for all species. The transport terms are obtained, including ion species diffusion, in the absence of magnetic field. In Ref. 18, the electron and ion transport theories are thoroughly developed for a two ion species plasma with disparate masses. The authors solve the variational problem by expanding the density distribution in generalized Laguerre polynomials of order $3 / 2$, following the formalism of Helander and Sigmar. ${ }^{21}$ The calculation is reduced to two independent "Spitzer" problems for electrons and light ions. The results for the electron transport theory are in agreement with the results derived in Ref. 17, where a generalization of Braginskii electron fluid description ${ }^{22}$ is performed for plasmas with multiple ion species, and therefore used a different set of thermodynamic forces and fluxes that lead to a different variational principle. In more recent publications, ${ }^{19,20}$ Simakov and Molvig developed the ion transport theory for an unmagnetized collisional plasma with multiple ion species of arbitrary mass. The fluid equations for multi-component plasmas are closed with the expressions derived therein for individual ion species drift velocities, total ion heat flux, and viscosity. They followed a generalization of the Braginskii ion fluid description, which agrees with the results previously obtained for two ion species with disparate masses in Ref. 18.

We apply the theory developed in Refs. 19 and 20 in the second part of this paper to study mass diffusion at the ablated border. Since the transport terms have only been derived for an unmagnetized plasma, we isolate the hydrodynamic problem and disregard the magnetic field evolution in this part. This paper is therefore organized as follows: In Sec. II, the problem is presented, and the governing equations are discussed not taking into account mass diffusion. They can be reduced to a system of two partial differential equations for temperature and magnetic field in which the solution presents a self-similar structure. In Sec. III, the results are discussed and the effect of the liner material on the thermal and magnetic flux losses is studied. In Sec. IV, the problem is formulated again taking into account mass diffusion. The governing equations can be reduced to a system of two independent equations for temperature and fuel concentration, whose solution also presents a self-similar nature. In Sec. V, the results with mass diffusion are presented and in Sec. VI, conclusions are drawn.

\section{GOVERNING EQUATIONS WITHOUT MASS DIFFUSION}

We consider at $t=0$ a hot plasma medium at rest with a uniform temperature $T_{0}$, ion particle density $n_{0}$, and thermal pressure $p_{0}$ occupying the semi-infinite space $x>0$. It is initially magnetized with a uniform magnetic field $\vec{B}_{0}=B_{0} \vec{e}_{z}$. The hot plasma is in contact at $x=0$ with a cold denser unmagnetized plasma $\left(T \ll T_{0},|\vec{B}| \ll B_{0}\right)$ that extends towards $x<0$, and they are in mechanical equilibrium (same pressure). Both plasmas are fully ionized and made of a different material, that is, different atomic number $Z$. They crudely represent the fuel, $Z=Z_{1}$, and the liner, $Z=Z_{2}$, respectively. Hereinafter, we consider that the fuel is made of deuterium, $Z_{1}=1$, and we take $Z_{2}$ as a free parameter. We let the system evolve for $t>0$. As a consequence of thermal conduction, the liner material ablates, compresses the fuel, and cools it down, while the magnetic field is subjected to convection and diffusion. The region $x>0$, which stands for the hot spot, is therefore formed of two plasmas of different atomic numbers $Z$ : ablated liner material and fuel, with the ablated border being the interface between them, see Fig. 1. The hot spot-liner interface, $x=0$, represents consequently an ablation front. Since the liner density is large, the ablation velocity is small, ${ }^{23}$ and we consider that the ablation front does not propagate much into the liner and stays at $x=0$ for all time. The liner (cold dense plasma) stays at rest and unmagnetized since its thermal conductivity, $\chi \sim T^{5 / 2}$, is low and the magnetic diffusivity, $D_{m} \sim T^{-3 / 2}$, is high (it can be considered as a magnetic isolant); neither heat flux nor electrical currents can take place in it.

We therefore focus on the dynamics of the plasmas in the hot spot, $x>0$. Since the fuel is preheated before compression in MagLIF implosions and the motion is subsonic, ${ }^{1}$ we assume low Mach number and we take a large thermal to magnetic pressure ratio: $\beta=8 \pi p_{0} / B_{0}^{2} \gg 1$. Due to the geometry of the problem, the only independent variables are the streamwise direction $x$ and time $t$, the plasma ion velocity is one-dimensional $\vec{v}=v(x, t) \vec{e}_{x}$, and the magnetic field stays perpendicular to the motion $\vec{B}=B(x, t) \vec{e}_{z}$. Quasi-neutrality, same ion and electron temperatures, and ideal gas hypotheses are assumed, and we take the adiabatic index $\gamma=5 / 3$. The evolution of the ion particle density $n$, thermal pressure $p=p_{e}+p_{i}$, temperature $T$, ion velocity $v$, and magnetic field $B$ is governed by the ion continuity, plasma momentum, and energy conservation equations neglecting plasma viscosity, together with Faraday's law (induction equation) and the equation of state, which for a small Mach number and large $\beta$ limit read

$$
\frac{\partial n}{\partial t}+\frac{\partial}{\partial x}(n v)=0
$$




$$
\begin{gathered}
\frac{\partial p}{\partial x}=0, \\
\frac{1}{\gamma-1} \frac{\partial p}{\partial t}+\frac{\partial}{\partial x}\left(\frac{\gamma}{\gamma-1} p v\right)=\frac{\partial}{\partial x}(\underbrace{\chi_{\perp} \frac{\partial T}{\partial x}}_{\text {Cond. }}), \\
\frac{\partial B}{\partial t}+\frac{\partial}{\partial x}(v B)=\frac{\partial}{\partial x}(\underbrace{D_{m \perp} \frac{\partial B}{\partial x}}_{\text {Joule }}+\underbrace{\frac{c \beta_{\wedge}^{u T}}{Z e n} \frac{\partial T}{\partial x}}_{\text {Nernst }}), \\
p=(1+Z) n T .
\end{gathered}
$$

We use Braginskii's expressions and notations for the transport coefficients, ${ }^{22}$ with a constant value equal to 7 for the Coulomb logarithm $\lambda$. The coefficient $\chi_{\perp}=\chi_{\perp}^{e}+\chi_{\perp}^{i}$ stands for electron plus ion conductivities, $\beta_{\wedge}^{u T}$ refers to the transport coefficient for the Nernst effect, and $D_{m \perp}$ $=\alpha_{\perp} c^{2} / 4 \pi e^{2} Z^{2} n^{2}$ is the magnetic diffusion coefficient appearing in the Joule dissipation. They are given by Eqs. (4.37), (4.40), (4.35), and (4.34) in Ref. 22, respectively. The momentum equation (2) is reduced to isobaricity: thermal pressure is constant and given by the initial conditions, $p=p_{0}$. Plasma energy equation (3) is thus reduced to the balance between enthalpy convection and thermal conduction. It can be integrated once, yielding an explicit relation for the ion velocity

$$
v=\frac{\gamma-1}{\gamma p_{0}} \chi_{\perp} \frac{\partial T}{\partial x} .
$$

Plasma velocity is therefore proportional to the thermal conduction heat flux $q=-\chi_{\perp} \partial T / \partial x$. As discussed in Ref. 8, in the integration of the equation of energy one should add a constant of integration $v_{\infty}$, which happens to be zero if the liner is modeled as a cold dense plasma. We impose zero heat flux at the liner interface since the heat conduction losses are recycled back into the hot spot via the ablated material. ${ }^{23,24}$

The position of the ablated border, denoted by $x_{b}(t)$, has to be determined self-consistently. It is determined by applying the fluid surface condition ${ }^{8}$

$$
\frac{\mathrm{d} x_{b}}{\mathrm{~d} t}=v\left(x_{b}, t\right) .
$$

In this section, we consider that both plasmas are immiscible (no atomic mass diffusion); hence, $x_{b}(t)$ represents also a contact discontinuity. Consequently, the atomic number $Z$ takes the value $Z=Z_{2}$ (liner material) at $x<x_{b}(t)$ and $Z=Z_{1}$ (fuel) at $x>x_{b}(t)$.

The system of governing equations (1), (4), and (6) has to be completed with the appropriate boundary conditions. We impose that far from the liner, $x \rightarrow \infty$, the plasma recovers the initial fuel state $n=n_{0}, T=T_{0}$, and $B=B_{0}$. At the interface with the liner, $x=0$, we require that the plasma temperature and magnetic field be equal to the temperature and magnetic field values at the liner, that is, $T / T_{0} \rightarrow 0$ and $B / B_{0} \rightarrow 0$.

In addition to the boundary conditions, we demand that temperature, magnetic field, and ion velocity be continuous at the ablated border. The latter condition implies continuity of the heat flux $q$. According to Eq. (5), the ion particle density is discontinuous, and it satisfies the relation

$$
\left(Z_{2}+1\right) n\left(x_{b}^{-}, t\right)=\left(Z_{1}+1\right) n\left(x_{b}^{+}, t\right) .
$$

We also introduce the mass density as $\rho=m_{i} n$, with $m_{i}$ being the ion mass. For simplicity, we consider that in both fuel and liner materials, the mass number doubles the atomic number, and we take the ion mass $m_{i}$ to be related to the proton mass $m_{p}$ through $m_{i}=2 Z m_{p}$. The former jump condition turns into

$$
\frac{Z_{2}+1}{Z_{2}} \rho\left(x_{b}^{-}, t\right)=\frac{Z_{1}+1}{Z_{1}} \rho\left(x_{b}^{+}, t\right) .
$$

Notice that for $Z_{1}=1, n\left(x_{b}^{-}, t\right) \leq n\left(x_{b}^{+}, t\right)$ but $\rho\left(x_{b}^{-}, t\right)$ $\geq \rho\left(x_{b}^{+}, t\right)$.

Finally, the induction equation (4) forces the sum of the Joule dissipation plus the Nernst term $D_{m \perp} \partial B / \partial x$ $+\left(c \beta_{\wedge}^{u T} / Z e n\right) \partial T / \partial x$ to be continuous through the ablated border.

\section{A. Normalization and self-similarity}

The ion particle density, temperature, and magnetic field in the hot spot are normalized with their initial value in the fuel: $\sigma=n / n_{0}, \theta=T / T_{0}$, and $\phi=B / B_{0}$. Ion density is related to temperature through the equation of state (5), yielding $\sigma=\left(Z_{1}+1\right) /(Z+1) \theta$. The ion mass density is likewise normalized $\varrho=\rho / \rho_{0}$, with $\rho_{0}=2 Z_{1} m_{p} n_{0}$. The thermal conductivity coefficient is normalized with the unmagnetized electron conductivity, $\chi_{\perp}=\bar{K} T_{0}^{5 / 2} \theta^{5 / 2} \mathcal{P}_{c}\left(x_{e} ; Z\right)$, where

$$
\bar{K} \equiv \frac{Z n \tau_{e} \gamma_{0}}{m_{e} T^{3 / 2}}=\frac{3 \gamma_{0}}{4 \sqrt{2 \pi m_{e}} e^{4} \lambda Z}
$$

is the constant factor in Spitzer conductivity, and $\tau_{e}, m_{e}$ are the electron collision time and mass, respectively. The thermoelectric transport coefficient is proportional to the thermal conductivity coefficient $c \beta_{\wedge}^{u T} /$ Zen $=\left[(\gamma-1) \chi_{\perp} / \gamma p_{0}\right] \quad B \mathcal{P}_{n}\left(x_{e} ; Z\right)$, while the magnetic diffusion coefficient is written as $D_{m \perp}$ $=\bar{D} T_{0}^{-3 / 2} \theta^{-3 / 2} \mathcal{P}_{d}\left(x_{e} ; Z\right)$, with

$$
\bar{D} \equiv \frac{c^{2} m_{e} T^{3 / 2} \alpha_{0}}{4 \pi e^{2} Z n \tau_{e}}=\frac{c^{2} \sqrt{2 m_{e}} \lambda e^{2} \alpha_{0} Z}{3 \sqrt{\pi}}
$$

being a diffusivity constant. Notice that the conductivity and diffusivity constants are functions of the atomic number: $\bar{K}(Z), \bar{D}(Z)$. The terms $\mathcal{P}_{c}, \mathcal{P}_{n}$, and $\mathcal{P}_{d}$ account for the effect of magnetization on the transport coefficients, see Fig. 2. They are rational functions of the electron Hall parameter (electron cyclotron frequency times the electron collision time)

$$
x_{e}=\omega_{e} \tau_{e}=\frac{e B}{m_{e} c} \tau_{e},
$$

and the atomic number $Z$, and read 

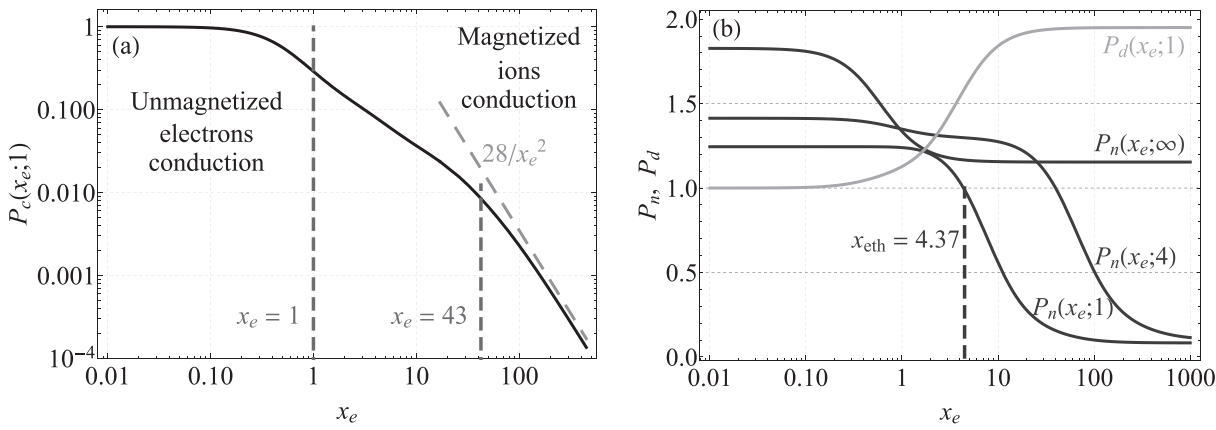

FIG. 2. Effect of magnetization on the transport coefficients: thermal conduction $\mathcal{P}_{c}\left(x_{e} ; Z\right)$, Nernst term $\mathcal{P}_{n}\left(x_{e} ; Z\right)$, and Joule dissipation $\mathcal{P}_{d}\left(x_{e} ; Z\right)$.

$$
\begin{aligned}
\mathcal{P}_{c}\left(x_{e} ; Z\right)= & \frac{\gamma_{1}^{\prime} x_{e}^{2}+\gamma_{0}^{\prime}}{\gamma_{0} \Delta_{e}}+\frac{1}{Z^{3}} \sqrt{\frac{2 m_{e}}{m_{i}}} \frac{2 x_{i}^{2}+2.645}{\gamma_{0} \Delta_{i}}, \\
\mathcal{P}_{n}\left(x_{e} ; Z\right)= & \frac{Z+1}{Z} \frac{\gamma}{\gamma-1} \\
& \times \frac{\beta_{1}^{\prime \prime} x_{e}^{2}+\beta_{0}^{\prime \prime}}{\gamma_{1}^{\prime} x_{e}^{2}+\gamma_{0}^{\prime}+\frac{1}{Z^{3}} \sqrt{\frac{2 m_{e}}{m_{i}}} \frac{\Delta_{e}}{\Delta_{i}}\left(2 x_{i}^{2}+2.645\right)}, \\
\mathcal{P}_{d}\left(x_{e} ; Z\right)= & \frac{1-\frac{\alpha_{1}^{\prime} x_{e}^{2}+\alpha_{0}^{\prime}}{\Delta_{e}}}{\alpha_{0}},
\end{aligned}
$$

with $\Delta_{e}=x_{e}^{4}+\delta_{1} x_{e}^{2}+\delta_{0}$ and $\Delta_{i}=x_{i}^{4}+2.70 x_{i}^{2}+0.677$, and $x_{i}=\omega_{i} \tau_{i}=x_{e} \sqrt{2 m_{e} / m_{i}} / Z$ standing for the ion magnetization. Finally, the coefficients $\gamma_{0}, \alpha_{0}, \gamma_{1}^{\prime}$ along with others are functions of the atomic number and are given in Braginskii $^{22}$ for $Z=1,2,3,4$ and $Z \rightarrow \infty$.

In an unmagnetized plasma, $x_{e} \ll 1$, thermal conduction is mainly due to electrons because of their small mass and $\mathcal{P}_{c} \approx 1$. However, the electrons get magnetized for lower magnetic field intensities compared to the ions $\left(x_{e}=1\right.$ with respect to $x_{i}=1 \Rightarrow x_{e}=Z \sqrt{m_{i} / 2 m_{e}} \approx 43 Z^{3 / 2}$ ), and when electron conduction is suppressed due to magnetization, the ions carry the heat transport. For large magnetization values, $x_{e}>Z \sqrt{m_{i} / 2 m_{e}}$, the ions also get magnetized and the thermal conductivity decreases asymptotically as $\mathcal{P}_{c} \sim x_{e}^{-2}$.

The electron Hall parameter anywhere in the hot spot, $x_{e}$, can be written as a function of its initial value in the fuel, the atomic number $Z$, and the dimensionless temperature and magnetic field profiles

$$
x_{e}=x_{e 0} \frac{Z+1}{Z^{2}} \frac{Z_{1}^{2}}{Z_{1}+1} \phi \theta^{5 / 2} .
$$

We define the magnetic Lewis number $\mathrm{Le}_{\mathrm{m}}$ as the ratio between the thermal and magnetic diffusivities in the fuel, defined, on their part, as

$$
\kappa_{0} \equiv \frac{(\gamma-1) \bar{K}\left(Z_{1}\right) T_{0}^{7 / 2}}{\gamma p_{0}} \approx \frac{40500}{\lambda} \frac{T_{0}^{5 / 2}}{\rho_{0}} \mathrm{~cm}^{2} / \mathrm{s}
$$

and

$$
\nu_{m 0} \equiv \bar{D}\left(Z_{1}\right) T_{0}^{-3 / 2} \approx \frac{13.33 \lambda}{T_{0}^{3 / 2}} \mathrm{~cm}^{2} / \mathrm{s}
$$

respectively. It reads

$$
\mathrm{Le}_{\mathrm{m}} \equiv \frac{\kappa_{0}}{\nu_{m 0}} \approx \frac{3038}{\lambda^{2}} \frac{T_{0}^{4}}{\rho_{0}}
$$

In the practical formulas here and below, the temperature is expressed in $\mathrm{keV}$ and the mass density in $\mathrm{g} / \mathrm{cc}$. The magnetic Lewis number can also be expressed in terms of the pressure ratio $\beta$ and the initial electron Hall parameter $x_{e 0}$

$$
\mathrm{Le}_{\mathrm{m}} \equiv \frac{\kappa_{0}}{\nu_{m 0}}=\frac{\gamma-1}{2 \gamma} \frac{Z_{1}^{2}}{\left(Z_{1}+1\right)^{2}} \frac{\gamma_{0}\left(Z_{1}\right)}{\alpha_{0}\left(Z_{1}\right)} \beta x_{e 0}^{2} \approx 0.31 \beta \mathrm{x}_{\mathrm{e} 0}^{2} .
$$

The initial value problem proposed lacks a characteristic length and velocity, and the ablated border position $x_{b}(t)$ is an eigenvalue of the problem. Therefore, the solution to (1), (4), and (6) is sought under the form of a self-similar diffusive wave, ${ }^{25}$ as done in Refs. 7 and 8 . We introduce, consequently, the independent self-similar variable $\eta \geq 0$

$$
\eta=\frac{x}{\sqrt{\kappa_{0} t}} .
$$

Consistently, the plasma ion velocity is scaled self-similarly

$$
V(\eta)=2 \sqrt{\frac{t}{\kappa_{0}}} v(x, t)
$$

which, by the use of the equation of energy (6), can be linked to temperature and magnetic field profiles as

$$
V=2 \bar{K}_{R} \mathcal{P}_{c} \theta^{5 / 2} \frac{\mathrm{d} \theta}{\mathrm{d} \eta} .
$$

Here, $\bar{K}_{R}$ stands for the Spitzer conductivity constant normalized with its value in the fuel

$$
\bar{K}_{R}(Z) \equiv \frac{\bar{K}(Z)}{\bar{K}\left(Z_{1}\right)}=\frac{\gamma_{0}(Z) Z_{1}}{\gamma_{0}\left(Z_{1}\right) Z} .
$$

The ion continuity equation (1) is rewritten as

$$
(V-\eta) \frac{\mathrm{d} \sigma}{\mathrm{d} \eta}+\sigma \frac{\mathrm{d} V}{\mathrm{~d} \eta}=0,
$$

which, by the use of the equation of state and inserting the expression for $V$, becomes

$$
\eta \frac{\mathrm{d} \theta}{\mathrm{d} \eta}+2 \theta^{2} \frac{\mathrm{d}}{\mathrm{d} \eta}\left(\bar{K}_{R} \mathcal{P}_{c} \theta^{3 / 2} \frac{\mathrm{d} \theta}{\mathrm{d} \eta}\right)=0
$$

The induction equation (4) likewise normalized gives 


$$
\begin{gathered}
-\eta \frac{\mathrm{d} \phi}{\mathrm{d} \eta}+2 \frac{\mathrm{d}}{\mathrm{d} \eta}\left[\bar{K}_{R} \mathcal{P}_{c}\left(1-\mathcal{P}_{n}\right) \theta^{5 / 2} \frac{\mathrm{d} \theta}{\mathrm{d} \eta} \phi\right] \\
=\frac{2}{\operatorname{Le}_{\mathrm{m}}} \frac{\mathrm{d}}{\mathrm{d} \eta}\left(\bar{D}_{R} \frac{\mathcal{P}_{d}}{\theta^{3 / 2}} \frac{\mathrm{d} \phi}{\mathrm{d} \eta}\right) .
\end{gathered}
$$

Similarly, $\bar{D}_{R}$ is the diffusivity constant normalized with its value in the fuel

$$
\bar{D}_{R}(Z) \equiv \frac{\bar{D}(Z)}{\bar{D}\left(Z_{1}\right)}=\frac{\alpha_{0}(Z) Z}{\alpha_{0}\left(Z_{1}\right) Z_{1}} .
$$

The conductivity ratio $\bar{K}_{R}(Z)$ is a decreasing function of $Z$, while $\bar{D}_{R}(Z)$ is an increasing function of $Z$.

Two opposite effects take part in the convection term in Eq. (24): magnetic field convection by the plasma motion, in the opposite direction to the conduction heat flux, and convection due to the Nernst term, in the same direction. The balance between both effects is given by the term $1-\mathcal{P}_{n}$. There is a threshold magnetization value $x_{e \text { th }}$ below which $\mathcal{P}_{n}>1$, the Nernst term is predominant, and the magnetic field is convected towards the liner. In the regions where $x_{e}$ $>x_{\text {eth }}$, the Nernst term is less effective, and the magnetic field is convected by the plasma towards the center of the hot spot. The value of $x_{e \text { th }}$ increases with $Z$, see Fig. 2(b). It takes the value $x_{\text {eth }}=4.37$ for $Z=1$, while $\mathcal{P}_{n}$ is always greater than 1 for $Z \rightarrow \infty$.

The problem is therefore reduced to solving Eqs. (23) and (24), which form a fourth order system of two ordinary differential equations for the normalized temperature and magnetic field profiles $\theta(\eta), \phi(\eta)$. They are coupled through the dependence of the thermal conductivity on the magnetization and need to be completed with the previously established boundary conditions

$$
\begin{aligned}
& \theta(0)=0, \quad \theta(\eta \rightarrow \infty)=1, \\
& \phi(0)=0, \quad \phi(\eta \rightarrow \infty)=1 .
\end{aligned}
$$

The normalized position of the ablated border, $\eta_{b}=x_{b} / \sqrt{\kappa_{0}} t$, has to be solved self-consistently. It is determined by Eq. (7), which transforms into

$$
\eta_{b}=V\left(\eta_{b}\right)
$$

The eigenvalue $\eta_{b}$ is thus proportional to the heat flux at the ablated border. Notice that, according to Eq. (22), the ion velocity has a stationary point there. The electron Hall parameter, Eq. (13), is discontinuous, and its value to the right $x_{e}^{+}=x_{e}\left(\eta_{b}^{+}\right)$is larger than its value to the left $x_{e}^{-}=x_{e}\left(\eta_{b}^{-}\right)$, related by

$$
x_{e}^{+}=x_{e}^{-} \frac{Z_{2}^{2}}{Z_{2}+1} \frac{Z_{1}+1}{Z_{1}^{2}} .
$$

The continuity of the heat flux and the Joule plus Nernst terms establishes the following conditions for the derivatives of the temperature and magnetic field:

$$
\left.\frac{\mathrm{d} \theta}{\mathrm{d} \eta}\right|_{\eta_{b}^{+}}=\left.\bar{K}_{R}\left(Z_{2}\right) \frac{\mathcal{P}_{c}^{-}}{\mathcal{P}_{c}^{+}} \frac{\mathrm{d} \theta}{\mathrm{d} \eta}\right|_{\eta_{b}^{-}},
$$

$$
\begin{aligned}
\left.\frac{\mathrm{d} \phi}{\mathrm{d} \eta}\right|_{\eta_{b}^{+}}= & \left.\operatorname{Le}_{\mathrm{m}} \frac{\theta_{b}^{4}}{\mathcal{P}_{d}^{+}} \bar{K}_{R}\left(Z_{2}\right) \mathcal{P}_{c}^{-} \frac{\mathrm{d} \theta}{\mathrm{d} \eta}\right|_{\eta_{b}^{-}}\left(\mathcal{P}_{n}^{-}-\mathcal{P}_{n}^{+}\right) \phi_{b} \\
& +\left.\bar{D}_{R}\left(Z_{2}\right) \frac{\mathcal{P}_{d}^{-}}{\mathcal{P}_{d}^{+}} \frac{\mathrm{d} \phi}{\mathrm{d} \eta}\right|_{\eta_{b}^{-}},
\end{aligned}
$$

where $\theta_{b}, \phi_{b}$ refer to their value at the ablated border, and the superscripts " + " and "-, on the transport polynomials refer to their evaluation to the left $\left(x_{e}^{-} ; Z_{2}\right)$ and to the right $\left(x_{e}^{+} ; Z_{1}\right)$ side of the ablated border, respectively. Since $\mathrm{Le}_{\mathrm{m}}$ is typically large in MagLIF implosions, the first term in the right-hand side of Eq. (31) is usually predominant. It is therefore the variation of the Nernst convection velocity with $Z$, that is, the factor $\left(\mathcal{P}_{n}^{-}-\mathcal{P}_{n}^{+}\right)$, what originates a steep thin layer at the ablated border where the magnetic field value changes drastically.

The system of governing equations consists thereby of Eqs. (23) and (24), together with boundary conditions (26), (27) and jump conditions (30), (31). It depends on three free parameters: the atomic number of the liner material $Z_{2}$, the magnetic Lewis number $\mathrm{Le}_{\mathrm{m}}$, and the electron Hall parameter of the unperturbed plasma $x_{e 0}$. Typically, high magnetic Lewis numbers, $\mathrm{Le}_{\mathrm{m}} \sim 100-10^{6}$, are attained throughout a MagLIF implosion. $^{7}$ Notice that the system of governing equations derived in Ref. 8 is recovered if we take $Z_{2}=Z_{1}=1$. Our final aim is to study mass ablation, thermal energy, and magnetic flux losses as a function of these parameters.

\section{B. Mass ablation, thermal energy, and magnetic flux losses}

An asymptotic analysis of Eqs. (23) and (24) performed for $\eta \ll 1$ reveals that the temperature and magnetic field profiles close to the liner take the form

$$
\left.\theta\right|_{\eta \ll 1}=s_{\theta} \eta^{2 / 5},\left.\quad \phi\right|_{\eta \ll 1}=\operatorname{Le}_{\mathrm{m}} s_{\phi} \eta^{8 / 5}
$$

The parameters $s_{\theta}$ and $s_{\phi}$ depend on $\mathrm{Le}_{\mathrm{m}}, x_{e 0}$, and $Z_{2}$, and are obtained by solving the complete problem with the boundary conditions far from the liner.

We define mass ablation $m$ and magnetic flux losses $\Phi$ per unit area in the hot spot as

$$
\begin{gathered}
m=\int_{0}^{x_{b}} \rho \mathrm{d} x, \\
\Phi=\int_{0}^{\infty}\left(B_{0}-B\right) \mathrm{d} x .
\end{gathered}
$$

Other quantities of interest are the thermal energy losses $\mathcal{E}$ and magnetic flux losses $\tilde{\Phi}$ in the fuel, defined as

$$
\begin{gathered}
\mathcal{E}=\int_{0}^{\infty} \frac{p_{0}}{\gamma-1} \mathrm{~d} x-\int_{x_{b}}^{\infty} \frac{p_{0}}{\gamma-1} \mathrm{~d} x=\frac{p_{0}}{\gamma-1} x_{b}, \\
\tilde{\Phi}=\int_{0}^{\infty} B_{0} \mathrm{~d} x-\int_{x_{b}}^{\infty} B \mathrm{~d} x .
\end{gathered}
$$

Following a similar analysis as performed in Ref. 8, these quantities are related to $s_{\theta}, s_{\phi}$, and $\eta_{b}$ as 

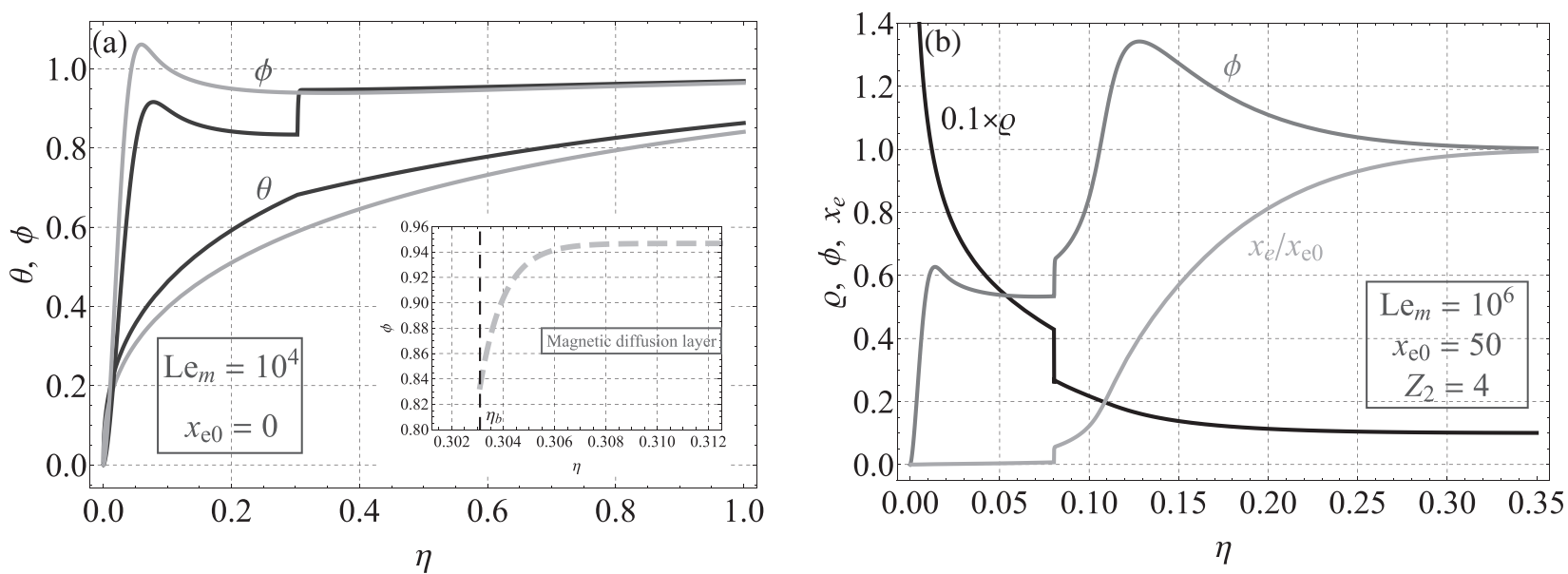

FIG. 3. (a) Temperature $\theta$ and magnetic field $\phi$ profiles for $\mathrm{Le}_{\mathrm{m}}=10^{4}, x_{e 0}=0$ and $Z_{2}=1$ (gray) and $Z_{2}=4$ (black). The magnetic field diffusion layer at $\eta$ $=\eta_{b}$ is compared to the analytic expression Eq. (44), which is shown in dashed lines in gray. (b) Mass density $\varrho$, magnetic field $\phi$, and Electron Hall parameter $x_{e}$ profiles for $\mathrm{Le}_{\mathrm{m}}=10^{6}, x_{e 0}=50$, and $Z_{2}=4$.

$$
\begin{gathered}
\frac{m}{\rho_{0} \sqrt{\kappa_{0} t}}=\frac{Z_{2}}{Z_{1}} \frac{Z_{1}+1}{Z_{2}+1} \bar{K}_{R}\left(Z_{2}\right) \frac{4}{5} s_{\theta}^{5 / 2}, \\
\frac{\Phi}{B_{0} \sqrt{\kappa_{0} t}}=\bar{D}_{R}\left(Z_{2}\right) \frac{16}{5} \frac{s_{\phi}}{s_{\theta}^{3 / 2}}, \\
\frac{(\gamma-1) \mathcal{E}}{p_{0} \sqrt{\kappa_{0} t}}=\eta_{b}, \\
\frac{\tilde{\Phi}}{B_{0} \sqrt{\kappa_{0} t}}=2 s_{b},
\end{gathered}
$$

with

$$
s_{b}=\left.\left(\bar{K}_{R} \mathcal{P}_{n} \mathcal{P}_{c} \theta^{5 / 2} \frac{\mathrm{d} \theta}{\mathrm{d} \eta} \phi+\frac{\mathcal{P}_{d} \bar{D}_{R}}{\operatorname{Le}_{\mathrm{m}} \theta^{3 / 2}} \frac{\mathrm{d} \phi}{\mathrm{d} \eta}\right)\right|_{\eta=\eta_{b}^{-}} .
$$

The magnetic flux in the fuel is thereby lost due to Nernst convection and magnetic diffusion through the ablated border, the former being predominant if $\mathrm{Le}_{\mathrm{m}}$ is large. The fuel thermal energy is lost due to thermal conduction at the ablated border, proportional to $\eta_{b}$, and is inverted into heating the ablated liner material.

\section{ANALYSIS AND RESULTS WITHOUT MASS DIFFUSION}

Numerical solutions of the governing equations are plotted in Fig. 3. Temperature and magnetic field profiles for the unmagnetized case and $\mathrm{Le}_{\mathrm{m}}=10^{4}$ are compared in Fig. 3(a) for two different liner materials, $Z_{2}=1$ (deuterium) and $Z_{2}$ $=4$ (beryllium). It can be seen that the plasma temperature at the hot spot is slightly higher in the second case. The magnetic field, convected by the Nernst term into the ablated material, is pushed against the liner and diffuses in a thin layer adjacent to it. When the liner is made of beryllium, the magnetic field experiences an abrupt decrease at the ablated border, $\eta_{b}=0.303$; as a consequence, the peak close to the liner attains lower values. According to the jump condition (31), this decrease corresponds to the dependence of the Nernst convection velocity on $Z$ through the polynomial

$\mathcal{P}_{n}\left(x_{e} ; Z\right)$. As seen in Fig. 2(b), $\mathcal{P}_{n}\left(x_{e} ; 4\right)>\mathcal{P}_{n}\left(x_{e} ; 1\right)$ for any $x_{e}$; hence, the Nernst convection velocity is stronger in the ablated material. In order to conserve the amount of magnetic field convected through the ablated border, $\phi$ is diffused in a thin magnetic diffusion layer at the fuel side and its value is reduced.

In Fig. 3(b), mass density, magnetic field, and magnetization profiles are shown for $\mathrm{Le}_{\mathrm{m}}=10^{6}, x_{e 0}=50$, and $Z_{2}$ $=4$. In the magnetized region, the Nernst term is suppressed and the magnetic field moves frozen into the plasma. Since the ablated material pushes the fuel inwards and compresses it, the magnetic field presents a second peak at the right side of the ablated border. At the left side, the electron Hall parameter is relatively small and Nernst convection dominates. Again, a magnetic diffusion layer takes place at the ablated border, $\eta_{b}=0.08$. In this position, both the magnetization and mass density present a discontinuity, given by Eqs. (29) and (9). The ablated border moves with negative acceleration $g=\mathrm{d}^{2} x_{b} / \mathrm{d} t^{2}=-\eta_{b} \sqrt{\kappa_{0}} / 4 t^{3 / 2}<0, \quad$ which implies that the light side of the ablated border (fuel) is pushing backwards the heavy side (liner material). This magnetohydrodynamic structure is susceptible to be Rayleigh-Taylor unstable and would deserve a more detailed study in a forthcoming paper.

\section{A. Magnetic diffusion layer at the ablated border}

In order to obtain the structure of the diffusion layer at the fuel side of the ablated border when $\mathrm{Le}_{\mathrm{m}}$ is large, we expand the self-similar variable as $\eta=\eta_{b}+\epsilon r$, with $\epsilon \ll 1$, $r>0$. We assume $\mathrm{d} \phi / \mathrm{d} r \sim O(1)$ in this layer and $\theta=$ $\theta_{b}+O(\epsilon), V=\eta_{b}+O\left(\epsilon^{2}\right)$. Inserting these Ansätze into the induction equation (24) yields to the leading order

$$
\frac{\mathrm{d}}{\mathrm{d} r}\left(\mathcal{P}_{n} \phi\right)=-\frac{2}{\epsilon \operatorname{Le}_{\mathrm{m}} \eta_{b} \theta_{b}^{3 / 2}} \frac{\mathrm{d}}{\mathrm{d} r}\left(\mathcal{P}_{d} \frac{\mathrm{d} \phi}{\mathrm{d} r}\right),
$$

where the electron Hall parameter in the transport polynomials shall be written as $x_{e}=x_{e 0} \theta_{b}^{5 / 2} \phi$. We obtain then the characteristic width of this layer 


$$
\epsilon=\frac{2}{\mathcal{P}_{n}\left(0 ; Z_{1}\right) \operatorname{Le}_{\mathrm{m}} \eta_{b} \theta_{b}^{3 / 2}},
$$

which scales with the magnetic Lewis number as $\epsilon \sim O\left(1 / \mathrm{Le}_{\mathrm{m}}\right)$. This layer is thinner than the diffusion layer adjacent to the liner, which scales as $O\left(1 / \sqrt{\mathrm{Le}_{\mathrm{m}}}\right) .{ }^{8}$

Equation (42) stands for a nonlinear equation of second order for $\phi$ that must be complemented with the boundary conditions: $\phi(r=0)=\phi_{b}$ and the magnetic field derivative given by Eq. (31). An analytical solution can be obtained in the unmagnetized limit, $x_{e 0} \ll 1$, in which case Eq. (42) takes the form $\mathrm{d}^{2} \phi / \mathrm{d} r^{2}+\mathrm{d} \phi / \mathrm{d} r=0$, and the solution reads

$$
\phi(r)=\phi_{b}\left[\frac{\mathcal{P}_{n}^{-}-\mathcal{P}_{n}^{+}}{\mathcal{P}_{n}^{+}}\left(1-e^{-r}\right)+1\right] .
$$

This analytical expression is compared to the numerical solution in Fig. 3(a), showing good agreement. Evaluating this profile far from the layer, $r \rightarrow \infty$, allows to obtain the magnetic field jump across it $\left(\phi^{r}\right.$ to the right and $\phi^{l}=\phi_{b}$ to the left), yielding

$$
\frac{\phi^{r}}{\phi_{b}}=\frac{\mathcal{P}_{n}^{-}}{\mathcal{P}_{n}^{+}}>0
$$

\section{B. Analytic solution for large $Z_{2}$}

In order to shed more light on the effect of the liner material, we solve the governing equations (23) and (24) in the limit of large liner atomic number $Z_{2}$ and $x_{e 0} \ll 1$. When $Z_{2}$ is large, the conductivity constant $\bar{K}$ in the ablated material is reduced, while the diffusivity constant $\bar{D}$ is increased. In this limit, we can write

$$
\begin{gathered}
\bar{K}_{R}\left(Z_{2} \gg 1\right)=\frac{3.94}{Z_{2}} \equiv \epsilon_{K}, \\
\bar{D}_{R}\left(Z_{2} \gg 1\right)=0.57 Z_{2} \equiv \frac{\alpha}{\epsilon_{K}},
\end{gathered}
$$

with $\epsilon_{K} \ll 1$ and $\alpha \equiv \alpha_{0}(\infty) \gamma_{0}(\infty) / \alpha_{0}(1) \gamma_{0}(1) \approx 2.27$. When $x_{e 0} \ll 1$, the continuity equation is uncoupled from the induction equation and can be solved independently. The transport polynomials take the value $\mathcal{P}_{c}(0 ; Z) \approx 1$, $\mathcal{P}_{d}(0 ; Z)=1$, and $\mathcal{P}_{n}(0 ; 1) \approx 1.24, \mathcal{P}_{n}(0 ; \infty) \approx 1.83$.

\section{Temperature profile}

In the ablated liner material, the small parameter $\epsilon_{K}$ can be absorbed in the continuity equation by scaling the independent variable as $\eta=\sqrt{\epsilon_{K}} s$. Letting $\theta_{l}$ be the temperature profile in this region, the continuity equation reads

$$
s \frac{\mathrm{d} \theta_{l}}{\mathrm{~d} s}+2 \theta_{l}^{2} \frac{\mathrm{d}}{\mathrm{d} s}\left(\theta_{l}^{3 / 2} \frac{\mathrm{d} \theta_{l}}{\mathrm{~d} s}\right)=0 .
$$

The temperature profile close to the liner takes the form $\theta_{l}(s \ll 1)=s_{\theta}^{\prime} s^{2 / 5}$, with $s_{\theta}^{\prime}=s_{\theta} \epsilon_{K}^{1 / 5}$. The solution has to satisfy the jump conditions at the ablated border. Scaling its position as $s_{b} \equiv \eta_{b} / \sqrt{\epsilon_{K}}$, it is determined by Eq. (28), which transforms into $2 \theta_{l}^{5 / 2} \mathrm{~d} \theta_{l} /\left.\mathrm{d} s\right|_{s_{b}}=s_{b}$. At this position, the temperature must be continuous, $\theta_{l}\left(s_{b}\right)=\theta_{f}\left(\eta_{b}\right)$, where $\theta_{f}(\eta)$ is the temperature profile in the fuel region. The temperature derivatives are linked through the jump condition (30), giving $\mathrm{d} \theta_{f} /\left.\mathrm{d} \eta\right|_{\eta_{b}}=\sqrt{\epsilon_{K}} \theta_{l b}^{\prime}$, where $\theta_{l b}^{\prime} \equiv \mathrm{d} \theta_{l} /\left.\mathrm{d} s\right|_{s_{b}}$. If we assume that $\mathrm{d} \theta_{l} / \mathrm{d} s \sim O(1)$, then the temperature derivative in the fuel is small of order $\sqrt{\epsilon_{K}}$, and the solution will not differ much from $\theta_{f} \approx 1$. Therefore, to the leading order, we can impose that $\theta_{l}\left(s_{b}\right)=1$. Consequently, the temperature profile at the ablated material $\theta_{l}$ can be solved independently of the fuel region in a small $\epsilon_{K}$ limit. The solution yields $s_{\theta}^{\prime}=1.21, s_{b}=0.81=2 \theta_{l b}^{\prime}$.

In the fuel region, the continuity equation reads

$$
\eta \frac{\mathrm{d} \theta_{f}}{\mathrm{~d} \eta}+2 \theta_{f}^{2} \frac{\mathrm{d}}{\mathrm{d} \eta}\left(\theta_{f}^{3 / 2} \frac{\mathrm{d} \theta_{f}}{\mathrm{~d} \eta}\right)=0 .
$$

Since the temperature derivatives are small, we expand the solution as $\theta_{f}=1-\sqrt{\epsilon_{K}} \theta_{f 1}+o\left(\sqrt{\epsilon_{K}}\right)$. Inserting this Ansatz in the previous equation and retaining the leading order terms allows to obtain the first correction, giving $\theta_{f 1}=\mathcal{C}[1-\operatorname{Erf}(\eta / 2)]$. The constant of integration $\mathcal{C}$ is obtained by applying the jump condition (30), yielding $\mathcal{C}=\sqrt{\pi} \theta_{l b}^{\prime} \approx 0.72$. The ion velocity and the Nernst velocity, proportional to the temperature derivative, are also small of order $O\left(\sqrt{\epsilon_{K}}\right)$.

From these results, we infer that the ablated mass and energy losses are written as

$$
\begin{gathered}
\frac{m}{\rho_{0} \sqrt{\kappa_{0} t}}=\frac{Z_{1}+1}{Z_{1}} \frac{4}{5} s_{\theta}^{15 / 2} \sqrt{\epsilon_{K}} \approx \frac{5.09}{\sqrt{Z_{2}}}, \\
\frac{(\gamma-1) \mathcal{E}}{p_{0} \sqrt{\kappa_{0} t}}=s_{b} \sqrt{\epsilon_{K}} \approx \frac{1.61}{\sqrt{Z_{2}}} .
\end{gathered}
$$

To conclude, in a large $Z_{2}$ limit, the ablated border steps back, the temperature profile is almost constant in the fuel and decreases in the thin ablated liner layer whose width scales as $O\left(1 / \sqrt{Z_{2}}\right)$, and both mass ablation and energy losses are reduced when $Z_{2}$ increases.

\section{Magnetic field profile}

We assume, as typically occurs in MagLIF, large $\mathrm{Le}_{\mathrm{m}}$. Consequently, diffusion can be initially neglected in the fuel. The magnetic field is convected by the plasma motion and the Nernst term, the latter being predominant in the unmagnetized case. We introduce $\delta_{l}=\mathcal{P}_{n}(0 ; \infty)-1 \approx 0.83$ and $\delta_{f}=\mathcal{P}_{n}(0 ; 1)-1 \approx 0.24$. The equation governing the magnetic field in the fuel, $\phi_{f}$, reads

$$
\eta \frac{\mathrm{d} \phi_{f}}{\mathrm{~d} \eta}+2 \delta_{f} \sqrt{\epsilon_{K}} \theta_{l b}^{\prime} \frac{\mathrm{d}}{\mathrm{d} \eta}\left(\mathrm{e}^{-\eta^{2} / 4} \phi_{f}\right)=0 .
$$

The solution satisfying $\phi_{f}(\infty)=1$, retaining terms up to $O\left(\sqrt{\epsilon_{K}}\right)$, gives $\phi_{f}=1-\sqrt{\epsilon_{K} \pi} \delta_{f} \theta_{l b}^{\prime}[1-\operatorname{Erf}(\eta / 2)]$. The magnetic field is barely perturbed since the convection velocity is small, of order $O\left(\sqrt{\epsilon_{K}}\right)$, and only the leading order $\phi_{f} \approx 1$ will be retained. Close to the ablated border, the thin diffusion layer described in Subsection III A takes place, its width given by Eq. (43) $\epsilon \sim O\left(1 / \mathrm{Le}_{\mathrm{m}} \sqrt{\epsilon_{K}}\right)$, and 
the magnetic field in this layer drops from $\phi_{f} \approx 1$ to $\phi_{b}=\left(1+\delta_{f}\right) /\left(1+\delta_{l}\right)=0.68$.

In the ablated plasma liner, the induction equation shall be rewritten as

$$
-s \frac{\mathrm{d} \phi_{l}}{\mathrm{~d} s}-2 \delta_{l} \frac{\mathrm{d}}{\mathrm{d} s}\left(\theta_{l}^{5 / 2} \frac{\mathrm{d} \theta_{l}}{\mathrm{~d} s} \phi_{l}\right)=\frac{2 \alpha}{\mathrm{Le}_{\mathrm{m}} \epsilon_{K}^{2}} \frac{\mathrm{d}}{\mathrm{d} s}\left(\theta^{-3 / 2} \frac{\mathrm{d} \phi_{l}}{\mathrm{~d} s}\right),
$$

where we assume $\mathrm{d} \phi_{l} / \mathrm{d} s \sim O(1)$. It can be seen that the magnetic Lewis number appropriate for this region is $\mathrm{Le}_{l}=\mathrm{Le}_{\mathrm{m}} \epsilon_{K}^{2} / \alpha$. As discussed at the end of this section, we will assume that $\mathrm{Le}_{l}$ is large too. Consequently, diffusion can be neglected in the main part of the ablated material region, while it is confined to a thin sub-layer close to the liner. Letting $\phi_{l o}$ denote the leading order solution (outer solution) of Eq. (53), and $w=2 \theta_{l}^{5 / 2} \mathrm{~d} \theta_{l} / \mathrm{d} s$, the former satisfies

$$
\frac{\mathrm{d} \phi_{l o}}{\mathrm{~d} s}=-\delta_{l} \phi_{l o} \frac{\mathrm{d} w / \mathrm{d} s}{s+\delta_{l} w}
$$

together with $\phi_{l o}\left(s_{b}\right)=\phi_{b}$. Solving this equation allows to obtain the shape of this profile close to the liner $\phi_{l o}(s \ll 1)=s_{\phi_{o}} s^{-2 / 5}$, with $s_{\phi_{o}}=0.28$. A thin sub-layer must then take place where the magnetic field diffuses and drops to its value at the liner, $\phi_{l}(0)=0$. In order to obtain the magnetic field in this sub-layer (inner solution $\phi_{l i}$ ), we expand Eq. (53) in the variable $s=\epsilon_{B} z$, with $\epsilon_{B} \ll 1$, and assume $\mathrm{d} \phi_{l i} / \mathrm{d} z \sim O(1)$. Requiring diffusion to be important gives the width of this boundary layer

$$
\epsilon_{B}=\sqrt{\frac{5}{2 \delta_{l} s_{\theta}^{\prime} \mathrm{Le}_{l}}}
$$

while the resulting equation for $\phi_{l i}$ is

$$
\frac{\mathrm{d}}{\mathrm{d} z}\left(z^{2 / 5} \phi_{l i}\right)+\frac{\mathrm{d}}{\mathrm{d} z}\left(\frac{1}{z^{3 / 5}} \frac{\mathrm{d} \phi_{l i}}{\mathrm{~d} z}\right)=0 .
$$

The solution satisfying the boundary condition $\phi_{l i}(0)=0$ is

$$
\phi_{l i}=Q\left[\int_{0}^{z} \hat{z}^{3 / 5} \exp \left(\hat{z}^{2} / 2\right) \mathrm{d} \hat{z}\right] \exp \left(-z^{2} / 2\right),
$$

where the constant of integration $Q$ is obtained by matching the inner solution $\phi_{l i}(z)$ for $z \rightarrow \infty$ with the outer $\phi_{l o}(s)$ for $s \rightarrow 0$, yielding $Q=s_{\phi_{o}}\left(2 \delta_{l} s_{\theta}^{\prime 5} \mathrm{Le}_{l} / 5\right)^{1 / 5}$. The solution in the ablated material region can be therefore expressed by an innerouter composite expansion: ${ }^{26} \phi_{l}(s)=\phi_{l i}\left(s / \epsilon_{B}\right)+\phi_{l o}(s)$ $-s_{\phi_{o}} s^{-2 / 5}$. The parameter $s_{\phi}$ yields, in this double limit large $Z_{2}$ and large $\mathrm{Le}_{1}, s_{\phi}=\epsilon_{K}^{6 / 5} s_{\phi_{o}} \delta_{l} s_{\theta}^{\prime 5} / 4 \alpha \approx 0.066 \epsilon_{K}^{6 / 5}$. From this analysis, we can derive the magnetic flux losses in the hot spot and in the fuel, reading

$$
\begin{gathered}
\frac{\Phi}{B_{0} \sqrt{\kappa_{0} t}}=\frac{4}{5} \delta_{l} s_{\phi_{o}} s_{\theta}^{7 / 2} \sqrt{\epsilon_{K}} \approx \frac{0.72}{\sqrt{Z_{2}}}, \\
\frac{\tilde{\Phi}}{B_{0} \sqrt{\kappa_{0} t}}=2\left(1+\delta_{l}\right) \theta_{l b}^{\prime} \phi_{b} \sqrt{\epsilon_{K}} \approx \frac{2.01}{\sqrt{Z_{2}}} .
\end{gathered}
$$

In the large $\mathrm{Le}_{l}$ limit, the main contribution to the magnetic flux losses in the fuel corresponds to the magnetic field convection through the ablated border due to the Nernst velocity, that is, the first term in the right-hand side of Eq. (41). The magnetic flux conservation both in the fuel and in the whole hot spot (fuel plus ablated material) is improved when $Z_{2}$ increases, following the same power law. This implies that the $64 \%[=100 \times(1-\Phi / \tilde{\Phi})]$ of the magnetic flux losses in the fuel are spent into magnetizing the ablated material, while the remaining $36 \%$ is lost through dissipation at the liner.

It is interesting to check the validity of this limit in realistic MagLIF conditions. We have assumed that the ablated liner material is fully ionized. The last ionization energy of aluminum, $Z_{2}=13$, corresponds to $2.3 \mathrm{keV}$, which is attained by the end of the implosion. At this stage, the magnetic Lewis number is large, ${ }^{7} \mathrm{Le}_{\mathrm{m}} \sim 10^{6}$; therefore, the assumption $\mathrm{Le}_{l} \sim \mathrm{Le}_{\mathrm{m}} / Z_{2}^{2} \gg 1$ is well satisfied.

\section{Results for mass ablation, thermal energy, and magnetic flux losses}

In Fig. 4, the integral quantities mass ablation, energy losses, and magnetic flux losses are shown as a function of the atomic number of the liner $Z_{2}$ for $\mathrm{Le}_{\mathrm{m}}=10^{9}$ and $x_{e 0}=0$. In order to obtain the integral quantities for any $Z_{2}$, we have interpolated the transport coefficients given by Braginskii ${ }^{22}$ using splines of order 3 , and taking $Z^{-1}$ as the interpolation variable. The conservation of both the thermal energy and the magnetic flux in the fuel is improved when $Z_{2}$ increases. On the contrary, mass ablation and magnetic flux losses in the hot spot attain a maximum for $Z_{2}=4, Z_{2}=12$, respectively, where they take the value 1.12 and 0.084 . Although large $Z_{2}$ values are physically meaningless, they are plotted to check the accuracy of the asymptotic laws derived in Subsection III B, which are also shown in the figure.

As stated in Subsection III B, increasing $Z_{2}$ lowers the thermal conductivity in the ablated material and the ion and Nernst velocities in all the hot spot. Consequently, heat flux at the ablated border is reduced, and less magnetic field is

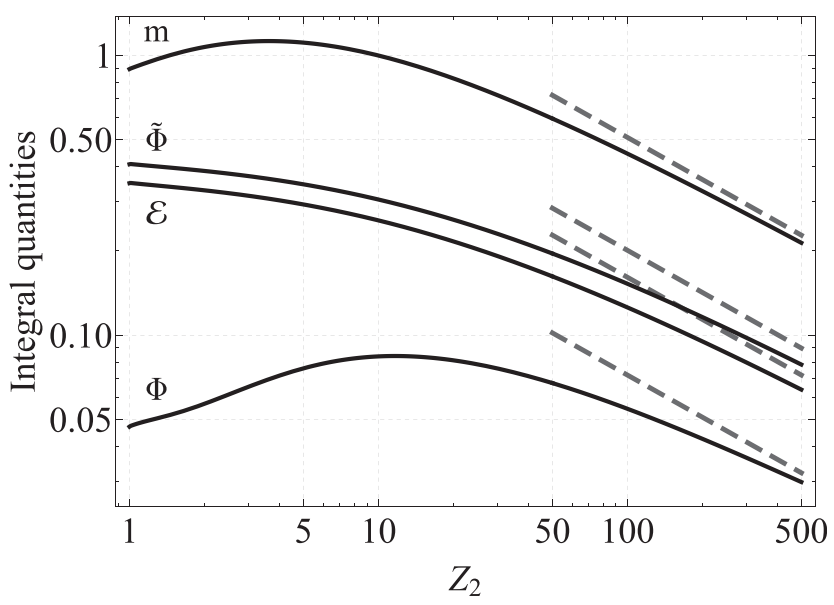

FIG. 4. Normalized mass ablation $m / \rho_{0} \sqrt{\kappa_{0} t}$, thermal energy losses $(\gamma-1) \mathcal{E} / p_{0} \sqrt{\kappa_{0} t}$, magnetic flux losses in the hot spot $\Phi / B_{0} \sqrt{k_{0} t}$, and magnetic flux losses in the fuel $\tilde{\Phi} / B_{0} \sqrt{k_{0} t}$ for $\mathrm{Le}_{\mathrm{m}}=10^{9}$ and $x_{e 0}=0$ as a function of the atomic number of the liner $Z_{2}$. The dashed lines correspond to the asymptotic laws derived in the large $Z_{2}$, Le $_{l}$ limit, Eqs. (50), (51), (58), and (59). 

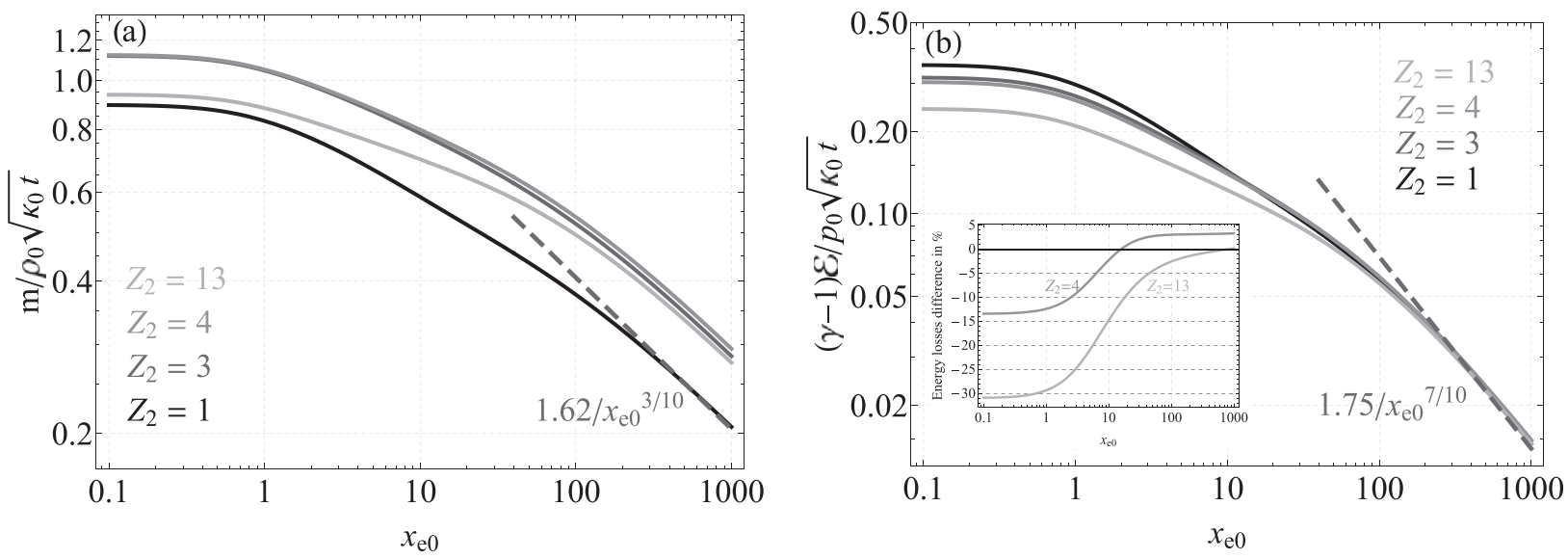

FIG. 5. Normalized mass ablation $m / \rho_{0} \sqrt{\kappa_{0} t}$ (a) and thermal energy losses $(\gamma-1) \mathcal{E} / p_{0} \sqrt{\kappa_{0} t}=\eta_{b}$ (b) for different liner materials. The curves keep a constant $\beta=1000$ when the magnetization is increased. In (b), the difference in percentage between the $Z_{2}=1$ and $Z_{2}=4, Z_{2}=13$ cases is plotted, computed as $100 \times\left(\left.\eta_{b}\right|_{Z_{2}=4}-\left.\eta_{b}\right|_{Z_{2}=1}\right) /\left.\eta_{b}\right|_{Z_{2}=1}$ and $100 \times\left(\left.\eta_{b}\right|_{Z_{2}=13}-\left.\eta_{b}\right|_{Z_{2}=1}\right) /\left.\eta_{b}\right|_{Z_{2}=1}$, respectively.

convected through it by the Nernst term. Both $\mathcal{E}$ and $\tilde{\Phi}$ decrease then with $Z_{2}$. One would expect the same trend for $m$ and $\Phi$, as the convection velocity is reduced and less magnetic field is accumulated at the liner. However, when $Z_{2}$ increases, the ions coming from the liner are heavier, and the magnetic Lewis number at the ablated liner, $\mathrm{Le}_{l} \sim \mathrm{Le}_{m} / Z_{2}^{2}$, is reduced, that is, the plasma becomes less conductive. These effects enhance $m$ and $\Phi$, respectively, and are predominant over the reduction of convection velocity for small $Z_{2}$, while the latter becomes more important for large $Z_{2}$, and therefore $m$ and $\Phi$ present a maximum.

The integral quantities mass ablation, thermal energy, and magnetic flux losses as a function of the fuel initial magnetization $x_{e 0}$ and for different liner materials (lithium $Z_{2}$ $=3$, beryllium $Z_{2}=4$, and aluminum $Z_{2}=13$ ) are shown in Figs. 5 and 6 . The curves are computed keeping a large $\beta$ $=1000$. In the same figures, asymptotic laws for large $x_{e 0}$ are plotted, some of which were obtained in Ref. 8 and adjusted by numerical fitting.

When the fuel is unmagnetized, low $x_{e 0}$, the mass and energy losses follow the same trend observed in Fig. 4: the ablated mass is enhanced for lithium and beryllium, it attains a maximum, and then decreases for higher $Z_{2}$ (aluminum).

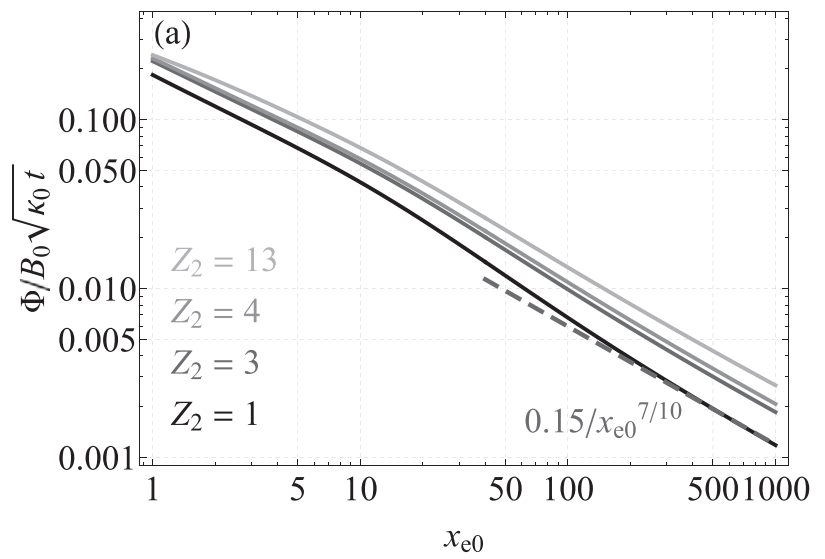

The energy losses, on their part, decrease monotonically with the atomic number of the liner. Magnetizing the fuel reduces the mass ablated and improves thermal insulation. The effect of $Z_{2}$ on thermal insulation becomes less important when the fuel is magnetized. It could even be inverted when the electron Hall parameter exceeds a certain threshold, see Fig. 5(b), but it barely enhances the energy losses by less than $5 \%$.

In Fig. 6, the magnetic flux losses in the hot spot and in the fuel are depicted. They are shown for $x_{e 0}>1$ since the magnetic Lewis number becomes relatively small when the magnetization is further reduced keeping a constant $\beta$ $=1000$ and is out of the range of application to MagLIF. Nevertheless, the arguments derived for the unmagnetized case still apply for moderate $x_{e 0}$. It can be seen that the magnetic flux losses in the hot spot are enhanced when $Z_{2}$ is increased up to the aluminum value, as the ablated material becomes more diffusive. This effect is preserved for all values of $x_{e 0}$. However, the magnetic flux conservation in the fuel, Fig. 6(b), follows a different trend. It is improved with $Z_{2}$ for moderate magnetizations due to the reduction of the Nernst velocity, but this effect diminishes when the magnetization is increased. It can even be inverted for a liner made

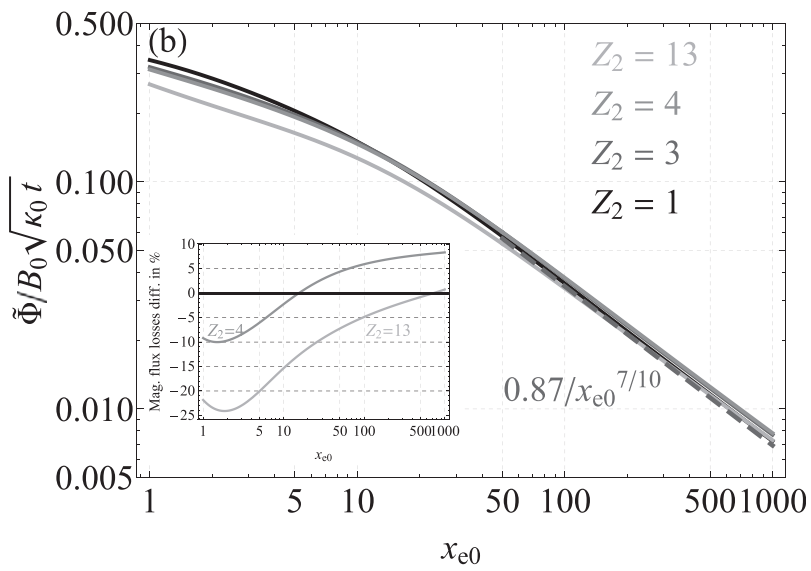

FIG. 6. Normalized magnetic flux losses computed in: (a) the hot spot $(x>0), \Phi / B_{0} \sqrt{\kappa_{0} t}$ and (b) the fuel $\left(x>x_{b}\right)$, $\tilde{\Phi} / B_{0} \sqrt{\kappa_{0} t}$, for different liner materials. The curves keep a constant $\beta=1000$ when the magnetization is increased. In (b), the difference in percentage between the $Z_{2}=1$ and $Z_{2}=4, Z_{2}=13$ cases is plotted, computed as $100 \times\left(\left.\tilde{\Phi}\right|_{Z_{2}=4}-\left.\tilde{\Phi}\right|_{Z_{2}=1}\right) /\left.\tilde{\Phi}\right|_{Z_{2}=1}$ and $100 \times\left(\left.\tilde{\Phi}\right|_{Z_{2}=13}-\left.\tilde{\Phi}\right|_{Z_{2}=1}\right) /\left.\tilde{\Phi}\right|_{Z_{2}=1}$, respectively. 
of beryllium for $x_{e 0}>15$, degrading the magnetic flux conservation by less than $10 \%$ compared to a liner made of dense deuterium.

\section{GOVERNING EQUATIONS WITH MASS DIFFUSION}

We formulate now the problem sketched in Fig. 1 taking into account mass diffusion through the ablated border. The hot spot is modeled as a two ion species plasma composed of deuterium (fuel) and liner material, and we assume that every ion species is fully ionized. The hydrodynamic description of a multiple species plasma, including a closed expression for the transport terms, is derived in Refs. 19 and 20. We follow the notation therein; therefore, the fuel (light species) is labeled with the subscript 1 , and the liner material (heavy species) with 2 and the electrons with $e$. We name $n_{k}$, with $k=\{1,2, e\}$, the particle number density, $\rho_{k}$ is the mass density, with $\rho_{k}=m_{k} n_{k}$, and $m_{k} \approx 2 Z_{k} m_{p}$ when $k$ refers to an ion species, $Z_{k}$ being its atomic number and $m_{p}$ the proton mass. We assume the same temperature for electrons and ions $T_{e}=T_{i}=T$. The partial pressure is $p_{k}=n_{k} T$, and $\vec{v}_{k}$ refers to the flow velocity. We define the plasma density $\rho=\sum_{k} \rho_{k} \approx \rho_{1}+\rho_{2}$, the total ion number density $n_{i}=n_{1}+n_{2}$, the total ion pressure $p_{i}=p_{1}+p_{2}=n_{i} T$, and the total plasma pressure $p=p_{i}+p_{e}$. Note that in Refs. 19 and $20, p$ denotes the total ion pressure, yet, we choose to use $p$ for the total plasma pressure to be consistent with the formulation in the first part of this paper, which at the same time inherits the original formulation in Ref. 8. The plasma ion velocity is defined as $\vec{v}=\left(\rho_{1} \vec{v}_{1}+\rho_{2} \vec{v}_{2}\right) / \rho$, and consequently the electron and ion drift velocities are expressed as $\vec{u}_{k}=\vec{v}_{k}-\vec{v}$. Again, although $\vec{u}$ refers to the plasma ion velocity and $\vec{v}_{k}$ to the drift velocities in Refs. 19 and 20, we choose to swap them and denote the plasma ion velocity as $\vec{v}$ to keep consistent with the first part of this paper. Finally, $x_{k}=n_{k} / n_{i}$ is the number density fraction of the ion species $k$, such that $x_{1}+x_{2}=1 ; y=\rho_{1} / \rho$ is the mass concentration of the fuel and $1-y$ is the mass concentration of the liner material.

As commented in the Introduction, we have to restrict the fuel liner mixing analysis to the unmagnetized plasma case, where the plasma motion is uncoupled from the magnetic field evolution. Studying mass diffusion in a magnetized plasma would require first to extend the transport theory of multi-component plasmas ${ }^{18-20}$ to take into account the effect of magnetic fields, which is out of the scope of this paper. In the absence of magnetic field, the Ampère's law, $\overrightarrow{0}=4 \pi \vec{j}+\partial \vec{E} / \partial t$, drives the electric field $\vec{E}$ to the value set by the ambipolarity condition $\vec{j}=0$, with $\vec{j}=e\left(Z_{1} n_{1} \vec{u}_{1}\right.$ $\left.+Z_{2} n_{2} \vec{u}_{2}-n_{e} \vec{u}_{e}\right)$ being the plasma current. As a consequence of the planar geometry and the absence of currents, the electron and ion velocities as well as the rest of the velocities introduced only present a streamwise component, and will be treated as scalars: $v_{k}, v$, and $u_{k}$.

The evolution of the plasma density $\rho$, pressure $p$, velocity $v$, and fuel concentration $y$ in the hot spot, $x>0$, is given by the total ion continuity, plasma momentum conservation, energy conservation, and fuel continuity equations, which in planar geometry and low Mach and high $\beta$ limit yield

$$
\begin{gathered}
\frac{\partial \rho}{\partial t}+\frac{\partial}{\partial x}(\rho v)=0 \\
\frac{\partial p}{\partial x}=0 \\
\frac{3}{2} \frac{\partial p}{\partial t}+\frac{\partial}{\partial x}\left[\frac{5}{2} p v+q_{e}+q_{i}+\frac{5}{2}\left(p_{1} u_{1}+p_{2} u_{2}+p_{e} u_{e}\right)\right]=E \cdot j \approx 0 \\
\rho \frac{\partial y}{\partial t}+\rho v \frac{\partial y}{\partial x}=-\frac{\partial}{\partial x}\left(\rho y u_{1}\right)
\end{gathered}
$$

where $q_{e}$ and $q_{i}$ are the electron and ion heat fluxes, respectively. The momentum conservation equation (61) reduces to isobaricity, $p=p_{0}$. In addition to these equations, we impose plasma quasi-neutrality, $n_{e}=Z_{1} n_{1}+Z_{2} n_{2}$. Besides, from the definition of plasma ion velocity, we have $\rho_{1} u_{1}+\rho_{2} u_{2}=0$, and the aforementioned ambipolarity condition gives $Z_{1} n_{1} u_{1}+Z_{2} n_{2} u_{2}-n_{e} u_{e}=0$. Using $n_{1}=\rho_{1} / m_{1}=\rho y / m_{1}$, any ion species or electron densities and drift velocities can be expressed in terms of $\rho, y$, and $u_{1}$, uniquely. The equation of state can therefore be written as

$$
p=p_{0}=\rho T\left[\left(1+Z_{1}\right) \frac{y}{m_{1}}+\left(1+Z_{2}\right) \frac{1-y}{m_{2}}\right] .
$$

With the isobaric assumption, plasma energy equation (62) can be integrated once, giving an explicit expression for the plasma velocity

$$
v=-\frac{2}{5 p_{0}}\left[q_{e}+q_{i}+\frac{5}{2}\left(p_{1} u_{1}+p_{2} u_{2}+p_{e} u_{e}\right)\right] .
$$

Expressing the plasma density $\rho$ in terms of $T$ and $y$ by means of Eq. (64), and making use of Eq. (65), we can reduce the governing equations to a system of two equations, (60) and (63), for $T(x, t)$ and $y(x, t)$. To close this system, we require the expressions for the electron and ion heat fluxes, $q_{e}$ and $q_{i}$, and the fuel drift velocity $u_{1}$. These relations are given in Refs. 19 and 20, and its derivation is briefly summarized in the Appendix. We make, therefore, use of Eqs. (A7), (A18), and (A16) to relate $q_{e}, q_{i}$, and $u_{1}$ to $T$ and $y$. Notice that the order of the system of differential equations is four. As boundary conditions, we impose that far from the liner, $x \rightarrow \infty$, we recover the initial state, $T=T_{0}$ and $y=1$, while at the linerhot spot interface, $x=0$, we have $T=0$ and $y=0$.

\section{A. Normalization and self-similarity}

Similarly to Subsection II A, we normalize temperature and plasma density with their initial value in the fuel: $\theta=T / T_{0}$ and $\varrho=\rho / \rho_{0}$. Again, the solution is sought under the form of a self-similar diffusive wave. ${ }^{25} \mathrm{We}$ introduce the independent self-similar variable $\eta=x / \sqrt{\kappa_{0} t}$, with

$$
\kappa_{0} \equiv \frac{2 \bar{K}_{e}\left(Z_{1}\right) T_{0}^{7 / 2}}{5 p_{0}} \approx \frac{40500}{\log \Lambda_{e e}} \frac{T_{0}^{5 / 2}}{\rho_{0}} \mathrm{~cm}^{2} / \mathrm{s}
$$

the thermal diffusivity being as likewise defined in Eq. (14). Consistently, the plasma velocity and the fuel drift velocities are scaled as

$$
V(\eta)=2 \sqrt{\frac{t}{\kappa_{0}}} v(x, t), \quad U_{1}(\eta)=2 \sqrt{\frac{t}{\kappa_{0}}} u_{1}(x, t) .
$$


The governing equations (60) and (63) are rewritten as

$$
\begin{gathered}
(V-\eta) \frac{\mathrm{d} \varrho}{\mathrm{d} \eta}+\varrho \frac{\mathrm{d} V}{\mathrm{~d} \eta}=0, \\
\varrho(V-\eta) \frac{\mathrm{d} y}{\mathrm{~d} \eta}=-\frac{\mathrm{d}}{\mathrm{d} \eta}\left(\varrho y U_{1}\right) .
\end{gathered}
$$

The dimensionless density $\varrho$ can be related to $\theta$ and $y$ through the equation of state (64), yielding

$$
\varrho=\frac{1}{\theta\left(y+\frac{Z_{2}+1}{Z_{1}+1} \frac{1-y}{\mu}\right)},
$$

with $\mu=m_{2} / m_{1}$. The dimensionless plasma velocity is obtained from Eq. (65), reading

$$
\begin{aligned}
V= & 2\left[\frac{\bar{K}_{e}\left(Z_{\mathrm{eff}}\right)+\bar{K}_{i}(v)}{\bar{K}_{e}\left(Z_{1}\right)}\right] \theta^{5 / 2} \frac{\mathrm{d} \theta}{\mathrm{d} \eta} \\
& -f_{\nu} \frac{y}{\left(Z_{1}+1\right) y+\left(Z_{2}+1\right) \frac{1-y}{\mu}} V_{1},
\end{aligned}
$$

with $f_{\nu}=\left[2 \hat{D}_{T 1}^{\prime} / 5 \hat{\Delta}_{11}^{\prime} x_{1}+Z_{1}+1-\left(Z_{2}+1\right) / \mu\right]$. Finally, the normalized fuel drift velocity is recovered from Eq. (A16) and gives

$$
U_{1}=-\frac{2}{\operatorname{Le}} \frac{\theta^{7 / 2}}{y}\left[\left(\mathcal{D}_{c}+\mathcal{D}_{p}\right) \frac{\mathrm{d} y}{\mathrm{~d} \eta}+\left(\mathcal{D}_{T_{e}}+\mathcal{D}_{T_{i}}\right) \frac{\mathrm{d} \log \theta}{\mathrm{d} \eta}\right],
$$

where Le stands for the Lewis number, typically defined in mass transfer problems as the ratio between thermal and mass diffusivities, $\kappa_{0}$ and $\nu_{0}$, respectively. ${ }^{27}$ The latter corresponds to the characteristic value of the coefficient relating fuel diffusion velocity and fuel mass concentration gradient in Eq. (A16), and reads

$$
\begin{aligned}
\nu_{0} & \equiv \frac{2 n_{1}}{\nu_{11} T^{3 / 2}} \frac{Z_{2}+1}{Z_{1}+1} \hat{\Delta}_{11}^{\prime}(0) \frac{T_{0}^{5 / 2}}{\rho_{0}} \\
& \approx \frac{1493}{\log \Lambda_{11}} P_{\mu} \frac{Z_{2}+1}{Z_{2}^{2}} \frac{T_{0}^{5 / 2}}{\rho_{0}} \mathrm{~cm}^{2} / \mathrm{s},
\end{aligned}
$$

TABLE I. Numerical values for deuterium fuel, $Z_{1}=1$, and liners made of lithium, $Z_{2}=3$, beryllium, $Z_{2}=4$, and aluminum, $Z_{2}=13$.

\begin{tabular}{lccc}
\hline \hline Liner material & Lithium & Beryllium & Aluminum \\
\hline Le & 85 & 111 & 323 \\
$\left.\eta_{b}\right|_{\text {Diff. }}$ & 0.325 & 0.313 & 0.249 \\
$\left.\eta_{b}\right|_{\text {Nodiff. }}$ & 0.315 & 0.304 & 0.245 \\
$\left.\frac{m}{\rho_{0} \sqrt{\kappa_{0} t}}\right|_{\text {Diff. }}$ & 1.15 & 1.16 & 0.973 \\
$\left.\frac{m}{\rho_{0} \sqrt{\kappa_{0} t}}\right|_{\text {Nodiff. }}$ & 1.12 & 1.13 & 0.951 \\
$\frac{m_{l f}}{\rho_{0} \sqrt{\kappa_{0} t}}$ & 0.0471 & 0.0422 & 0.0272 \\
$\left.\frac{m_{l f}}{\rho_{0} \sqrt{\kappa_{0} t}}\right|_{\text {B.L. }}$ & 0.0425 & 0.0388 & 0.0258 \\
$100 \times \frac{m_{l f}}{m}$ & $4.09 \%$ & $3.64 \%$ & $2.80 \%$ \\
$\epsilon_{d}$ & 0.0753 & 0.0682 & 0.0471 \\
$\frac{h_{\mathrm{D}}}{g t^{2}}$ & 0.926 & 0.871 & 0.756 \\
\hline \hline
\end{tabular}

with $P_{\mu}$ being an increasing function of the ion mass ratio $\mu$, given in Eq. (A22). It ranges from 0.595 for $\mu=1$ to 1.20 for $\mu \rightarrow \infty$.

The Lewis number is then

$$
\text { Le } \equiv \frac{\kappa_{0}}{\nu_{0}}=\frac{1}{5 \sqrt{2}} \frac{\gamma_{0}\left(Z_{1}\right) Z_{1}^{3}}{Z_{2}+1} \frac{1}{\hat{\Delta}_{11}^{\prime}(0)} \sqrt{\frac{m_{1}}{m_{e}}} \approx \frac{27}{P_{\mu}} \frac{Z_{2}^{2}}{Z_{2}+1}
$$

Notice that it only depends on the liner atomic number, and its value is shown in Table I for typical liner materials. The Lewis number is relatively large and becomes larger for higher $Z_{2}$, which implies that mass transport is less effective than heat transport. The motion of the plasma in the hot spot is therefore governed by the heat wave, and mass diffusion is confined within a thin layer placed at the ablated border.

The terms $\mathcal{D}_{c}, \mathcal{D}_{p}, \mathcal{D}_{T_{e}}$, and $\mathcal{D}_{T_{i}}$ are the dimensionless coefficients for concentration gradient diffusion, barodiffusion, electron thermo-diffusion, and ion thermodiffusion, respectively,

$$
\mathcal{D}_{c} \equiv \frac{\hat{\Delta}_{11}^{\prime}(y)}{\hat{\Delta}_{11}^{\prime}(0)} \frac{1-y+\frac{Z_{1}+1}{Z_{2}+1} \mu y}{(1-y+\mu y)^{2}},
$$

$$
\begin{aligned}
\mathcal{D}_{p} \equiv & \mathcal{D}_{c} y(1-y) \\
\times & \frac{\mu\left(Z_{2}-Z_{1}\right)^{2}}{Z_{2}\left(Z_{2}+1\right)\left(1-y+\frac{Z_{1}}{Z_{2}} \mu y\right)\left(1-y+\frac{Z_{1}+1}{Z_{2}+1} \mu y\right)}, \\
\mathcal{D}_{T_{e}} \equiv & \frac{\hat{\Delta}_{11}^{\prime}(y)}{\hat{\Delta}_{11}^{\prime}(0)} y(1-y)\left(1-y+\frac{Z_{1}+1}{Z_{2}+1} \mu y\right) \\
& \times \frac{Z_{1}}{Z_{2}} \frac{Z_{2}-Z_{1}}{(1-y+\mu y)\left(1-y+\frac{Z_{1}^{2}}{Z_{2}^{2}} \mu y\right)} \beta_{0}\left(x_{1}\right), \\
& \mathcal{D}_{T_{i}} \equiv \frac{\hat{D}_{T 1}^{\prime}(y)}{\hat{\Delta}_{11}^{\prime}(0)} \frac{1}{\mu}\left(1-y+\frac{Z_{1}+1}{Z_{2}+1} \mu y\right) .
\end{aligned}
$$

Notice that, as a consequence of isobaricity, both the electron and ion pressure gradient terms in Eq. (A13) can be expressed in terms of fuel mass concentration gradients. The sum of both effects is accounted for in $\mathcal{D}_{p}$ and is denoted hereinafter as baro-diffusion.

As will be explained in Subsection V A, concentration gradient diffusion and baro-diffusion are predominant for large Lewis numbers. Both coefficients are plotted in Fig. 7 for increasing $Z_{2}$. The former decreases with $Z_{2}$, while the latter increases. Baro-diffusion is zero in both pure liner $(y=0)$ and fuel $(y=1)$ limits, and it dominates over concentration gradient diffusion in most part of the layer when the liner atomic number is large. As can be seen in Fig. 7(b), the sum of both coefficients approaches $\mathcal{D}_{c}+\mathcal{D}_{p}=1-y$ when $Z_{2}$ is large.

The system of normalized governing equations consists, therefore, of Eqs. (68) and (69), with density given by Eq. (70), plasma velocity by Eq. (71), and fuel drift velocity by 

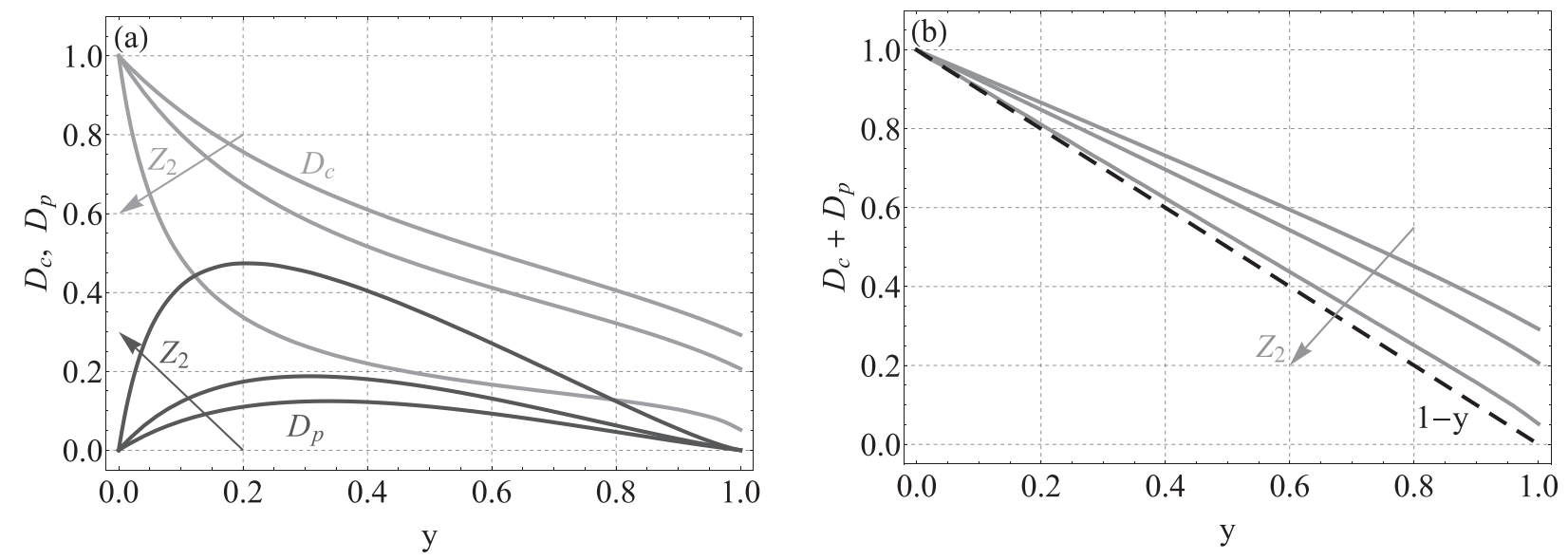

FIG. 7. Concentration gradient diffusion coefficient, $\mathcal{D}_{c}$, and baro-diffusion coefficient, $\mathcal{D}_{p}$, for $Z_{2}=3$ (lithium), $Z_{2}=4$ (beryllium), and $Z_{2}=13$ (aluminum), plotted separately in (a) and summed in (b).

Eq. (72). It only depends on the free parameter Le, or, equivalently, the liner atomic number.

\section{RESULTS WITH MASS DIFFUSION}

The numerical resolution of the normalized governing equations is shown in Fig. 8. Temperature, velocity, and mass diffusion rate profiles are plotted in Fig. 8(a) for deuterium fuel and beryllium liner. Temperature and velocity profiles are compared to the solution neglecting mass diffusion explained in the first part of this paper. Both solutions should be identical for an infinite Lewis number. It can be seen that, although the Lewis number for beryllium is not significantly large, $\mathrm{Le}=72$, the profiles are remarkably similar. This suggests that, in a MagLIF context, the hydrodynamic motion would not need to be solved self-consistently with the mass diffusion problem. It can rather be solved independently (immiscible plasmas), and mass diffusion be computed afterwards yielding a similar result. This argument is reinforced by the results shown in Table I, where the position of the ablated border and mass ablation are computed with and without mass diffusion, showing good agreement. The fuel mass diffusion rate, $-\varrho y U_{1}$, is relatively small and has a maximum at the ablated border.

The most significant difference between the diffusion and no diffusion solutions lies in the plasma velocity profile, which presents a bump at the ablated border. It is there where liner and fuel materials are in contact and mass diffusion takes place. As a consequence of the strong fuel concentration variations, pressure inhomogeneities arise, which locally accelerate the plasma. The plasma velocity is modified and convects fuel material in order to restore isobaricity.

Liner mass concentration profiles with and without mass diffusion are plotted in Fig. 8(b). Without diffusion, the liner concentration drops from 1 to 0 at the ablated border. When mass diffusion is taken into account, the ablated liner material penetrates into the fuel a relatively short distance compared to the characteristic thermal length, given by $\eta_{b}$. In Fig. 8(c), fuel mass concentration profiles are shown for different liner materials. It can be seen that the width of the diffusion layer shrinks when $Z_{2}$ increases, as the Lewis number becomes higher.

\section{A. Mass diffusion boundary layer}

When the Lewis number is large, mass diffusion is confined within a sharp boundary layer placed at the ablated border. We recall that the position of the ablated border is obtained by Eq. (28) and that velocity has null derivative there.

In order to obtain the structure of the thin diffusive layer, we expand the independent variable as $\eta=\eta_{b}+\epsilon_{d} s$, with $\epsilon_{d} \ll 1$. The fuel concentration varies from 0 to 1 in this region; hence, we assume $\mathrm{d} y / \mathrm{d} s \sim O(1)$. Since the layer is thin, the temperature does not vary significantly in it, and we make the isothermal approximation $\theta \approx \theta_{b}$, where $\theta_{b}$ stands for the temperature value at the ablated border. This hypothesis is consistent with the analysis of a diffusing gas-metal interface in a thermonuclear plasma made in Ref. 28, where the authors assumed both isobaric and isothermal conditions. Taking the first derivative in the equation of state (70), it can be obtained that $\mathrm{d} \varrho / \mathrm{d} s$ $\sim \mathrm{d} y / \mathrm{d} s \sim O(1)$; that is, density variation is important in this layer. According to Eq. (68), this variation forces the first derivative of the velocity, $\mathrm{d} V / \mathrm{d} \eta$, to be of order unity as soon as we move far from the ablated border while still being inside the layer. Consequently, we expand $V \approx \eta_{b}+\epsilon_{d} V_{c}(s)$, with $\mathrm{d} V_{c} / \mathrm{d} s \sim O(1)$. In the isothermal approximation, and defining $\mathcal{D}_{y} \equiv \mathcal{D}_{c}+\mathcal{D}_{p}$, the fuel drift velocity simplifies to

$$
U_{1}=-\frac{2 \theta_{b}^{7 / 2}}{\text { Le }} \frac{\mathcal{D}_{y}}{y} \frac{\mathrm{d} y}{\mathrm{~d} \eta}
$$

Notice that only concentration gradients and baro-diffusion drive mass mixing in the layer, while thermo-diffusion is negligible due to the smallness of the temperature derivatives compared to the fuel concentration derivatives. Inserting these expressions into diffusion equation (69), and forcing 

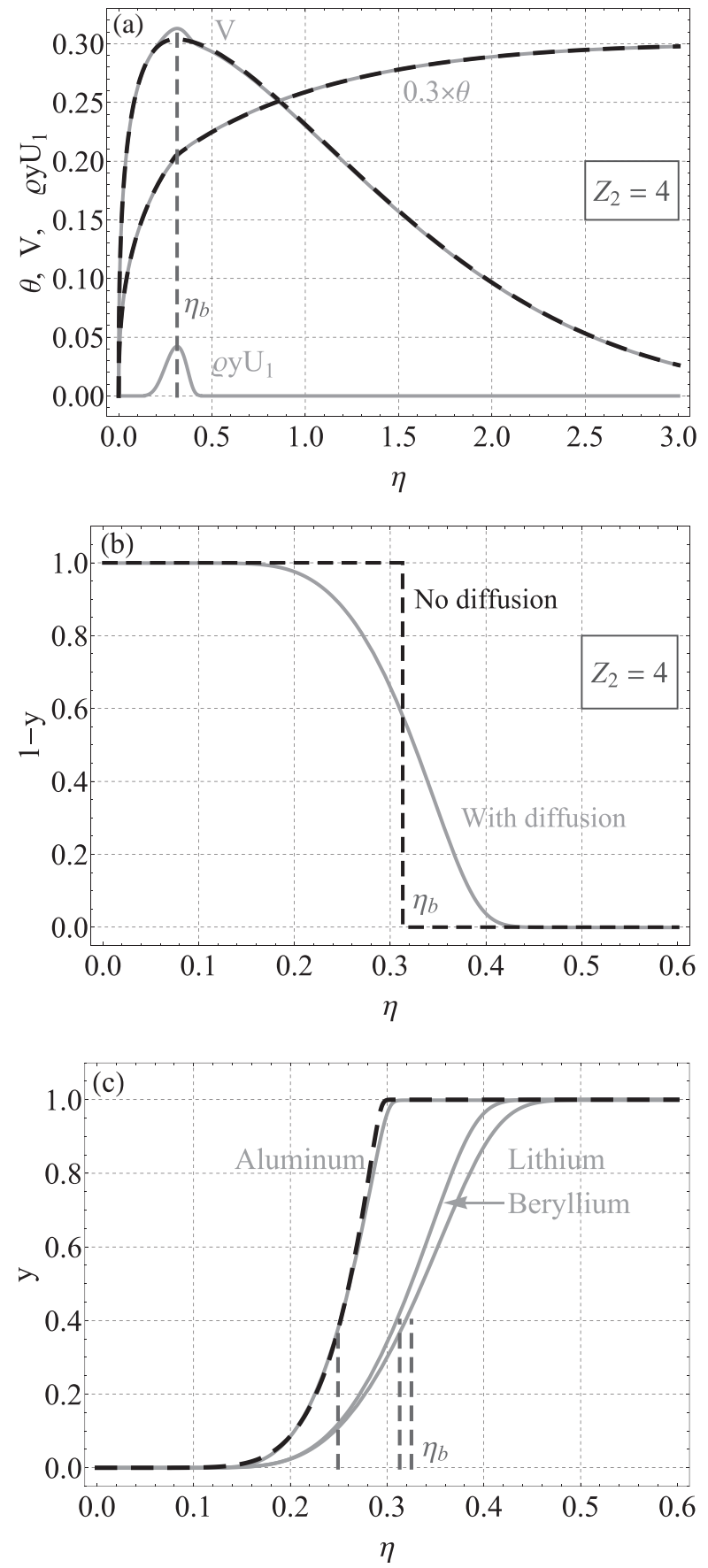

FIG. 8. (a) Normalized temperature $\theta$, velocity $V$, and fuel mass diffusion rate $-\varrho y U_{1}$ for a liner made of beryllium, depicted in solid gray lines. Temperature and velocity profiles are compared to the solution without mass diffusion, plotted in dashed black lines. (b) Beryllium mass concentration profile, $\rho_{2} / \rho=1-y$, in solid gray lines compared to the solution without mass diffusion, plotted in dashed lines. (c) Fuel mass concentration $y$ for different liner materials. The black dashed line is the fuel mass concentration profile for aluminum liner obtained from the boundary layer model derived in Subsection V A The position of the ablated border $\eta_{b}$ is marked in gray dashed lines.

the mass diffusion term to be important, we can obtain the width of the diffusion layer

$$
\epsilon_{d}=\sqrt{\frac{2 \theta_{b}^{7 / 2}}{\mathrm{Le}}} .
$$

The fuel drift velocity is therefore of order $O(1 / \sqrt{\mathrm{Le}})$

$$
U_{1}=-\sqrt{\frac{2 \theta_{b}^{7 / 2}}{\operatorname{Le}}} \frac{\mathcal{D}_{y}}{y} \frac{\mathrm{d} y}{\mathrm{~d} s} .
$$

Defining $a \equiv \mu\left(Z_{1}+1\right) /\left(Z_{2}+1\right)-1$, the continuity and diffusion equations (68), (69) take the form

$$
\begin{gathered}
\left(V_{c}-s\right) \frac{a}{1+a y} \frac{\mathrm{d} y}{\mathrm{~d} s}=\frac{\mathrm{d} V_{c}}{\mathrm{~d} s} \\
\left(V_{c}-s\right) \frac{1}{1+a y} \frac{\mathrm{d} y}{\mathrm{~d} s}=\frac{\mathrm{d}}{\mathrm{d} s}\left(\frac{D_{y}}{1+a y} \frac{\mathrm{d} y}{\mathrm{~d} s}\right) .
\end{gathered}
$$

As boundary conditions, we impose $y(s \rightarrow-\infty)=0$, $y(s \rightarrow \infty)=1$, and $V_{c}(s=0)=0$. These equations can be combined, yielding

$$
\left[a\left(\frac{\mathcal{D}_{y}}{1+a y} \frac{\mathrm{d} y}{\mathrm{~d} s}-\zeta\right)-s\right] \frac{1}{1+a y} \frac{\mathrm{d} y}{\mathrm{~d} s}=\frac{\mathrm{d}}{\mathrm{d} s}\left(\frac{\mathcal{D}_{y}}{1+a y} \frac{\mathrm{d} y}{\mathrm{~d} s}\right)
$$

where

$$
\zeta=\left.\frac{\mathcal{D}_{y}}{1+a y} \frac{\mathrm{d} y}{\mathrm{~d} s}\right|_{s=0}
$$

is an eigenvalue that has to be obtained self-consistently with $y(s)$ applying the former three boundary conditions. This eigenvalue is related to the fuel mass diffusion rate at the ablation front

$$
-\left.\varrho y U_{1}\right|_{s=0}=\sqrt{\frac{2 \theta_{b}^{3 / 2}}{\mathrm{Le}}}(a+1) \zeta .
$$

Finally, the first order correction to velocity can be obtained a posteriori from Eq. (82) as

$$
V_{c}=a\left(\frac{\mathcal{D}_{y}}{1+a y} \frac{\mathrm{d} y}{\mathrm{~d} s}-\zeta\right)
$$

The structure of the layer for an aluminum liner is plotted in Fig. 9. Since large Lewis number values imply large

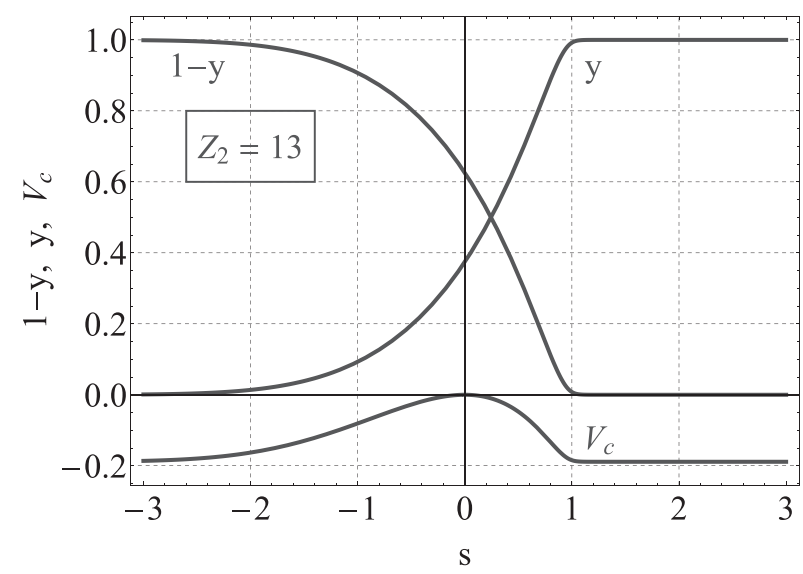

FIG. 9. Profiles in the boundary layer model. Fuel mass concentration, $y$, liner material mass concentration, $1-y$, and first order correction for velocity, $V_{c}$, for aluminum liner $\left(Z_{2}=13\right)$. 
$Z_{2}$, we use in this section the analytic expressions (A21) derived for a large $\mu$ ratio for the transport coefficients. It can be seen that the profiles are not symmetric. The asymmetry is introduced by the coefficient $\mathcal{D}_{y}$, plotted in Fig. 7 (b). The fuel mass concentration profile presents a well-defined wave front with clean fuel to the right at $s \approx 1$, and decreases exponentially to the left of the ablated border, where the fuel diffuses into the ablated material. Consequently, the parameter $\epsilon_{d}$ can be chosen as an accurate characterization of the width of the diffusion layer. The first order velocity correction $V_{c}$ corresponds to the bump appearing in the velocity profile in Fig. 8(a). The eigenvalue $\zeta$ gives $\{0.25,0.24,0.22,0.21\}$ for $Z_{2}=\{3,4,13, \infty\}$, respectively. Although not shown here, the profiles keep a similar structure for different $Z_{2}$. Particularly, the wave front becomes a sharp corner when $Z_{2} \rightarrow \infty$. The existence of a clear wave front was also discussed in the analysis made in Ref. 28. Comparisons of the boundary layer model with the complete solution are shown in Fig. 8(b), showing good agreement.

The analysis of this layer presents slight differences compared to the aforementioned study of a diffusing gasmetal interface in a thermonuclear plasma, Ref. 28. Although the expressions for the diffusion coefficients are the same (an equivalent notation taken from Ref. 18 is used therein), and the interface structure is also self-similar, the authors solved the mass diffusion problem without taking into account the hydrodynamic motion induced in the plasma. The governing equation of their diffusion layer, Eq. (9) in Ref. 28, is equivalent to Eq. (83) in this paper setting $V_{c}=0$. We find that similar results are obtained when the hydrodynamic motion of the plasma is not taken into account. Particularly, the eigenvalue $\zeta$ is only modified by $1 \%$.

\section{B. Liner material and fuel mixing}

We can characterize the fuel contamination by the amount of liner material that diffuses into it, defined as

$$
m_{l f}=\int_{x_{b}}^{\infty} \rho_{2} \mathrm{~d} x=\int_{x_{b}}^{\infty} \rho(1-y) \mathrm{d} x .
$$

Taking the time derivative and making use of the diffusion equation (63), we obtain

$$
\frac{\mathrm{d} m_{l f}}{\mathrm{~d} t}=-\left.\rho y u_{1}\right|_{x_{b}}=-\left.\frac{1}{2} \rho_{0} \sqrt{\frac{\kappa_{0}}{t}} \varrho y U_{1}\right|_{\eta_{b}},
$$

that is, fuel contamination is given by the mass diffusion rate at the ablated border, where it is maximum. If we integrate this expression in time, we have

$$
\frac{m_{l f}}{\rho_{0} \sqrt{\kappa_{0} t}}=-\left.\varrho y U_{1}\right|_{\eta_{b}} .
$$

We can use the boundary layer model, Eq. (86), to further develop this expression in a large Le limit

$$
\left.\frac{m_{l f}}{\rho_{0} \sqrt{\kappa_{0} t}}\right|_{\text {B.L. }}=\sqrt{\frac{2 \theta_{b}^{3 / 2}}{\mathrm{Le}}} \mu \frac{Z_{1}+1}{Z_{2}+1} \zeta .
$$

The liner material diffused into the fuel scales, therefore, as the inverse of the square root of the Lewis number. It is computed in Table I for different liner materials comparing the exact result, Eq. (90), with the boundary layer model, (91), giving similar results.

We can also compute the fuel mass diffused into the ablated liner, $m_{f l}=\int_{0}^{x_{b}} \rho_{1} \mathrm{~d} x=\int_{0}^{x_{b}} \rho y \mathrm{~d} x$, which can be proved to be equal to the liner material diffused into the fuel, $m_{f l}=m_{l f}$, by using Eqs. (60) and (63). This is a consequence of choosing the ablated border as the interface from which we compute fuel contamination.

As done in the analysis of the problem without mass diffusion, we compute the ablated liner material as

$$
m \equiv \int_{0}^{\infty} \rho_{2} \mathrm{~d} x=\int_{0}^{\infty} \rho(1-y) \mathrm{d} x .
$$

Performing a similar manipulation, we can express this quantity as

$$
\frac{m}{\rho_{0} \sqrt{\kappa_{0} t}}=\mu \frac{Z_{1}+1}{Z_{2}+1} \frac{\bar{K}_{e}\left(Z_{2}\right)}{\bar{K}_{e}\left(Z_{1}\right)} \frac{4}{5} s_{\theta}^{5 / 2},
$$

where $s_{\theta}$ is related to the temperature profile close to the liner as defined in Eq. (32). Note that this expression for mass ablation is equivalent to its counterpart in the problem without diffusion, Eq. (37).

It is interesting to compute the percentage of mass ablation that pollutes the liner, $100 \times m_{l f} / m$, shown in Table I. It decreases from $4.09 \%$ for lithium liner to $2.80 \%$ for aluminum liner.

As commented in Sec. III, the steep negative density gradient taking place at the ablated border may lead to a Rayleigh-Taylor instability (RTI), causing liner and fuel mixing. In order to compare fuel contamination caused by mass diffusion (microscopic mixing) to the one due to the turbulent motion following the RTI (macroscopic mixing), we roughly estimate the ratio $h_{\mathrm{D}} / h_{\mathrm{T}}$, where $h_{\mathrm{D}}=\epsilon_{d} \sqrt{\kappa_{0} t}$ is the width of the diffusion layer, and $h_{\mathrm{T}} \propto g t^{2}$ is the width of the turbulent mixing layer. The constant of proportionality depends on the particularities of the problem, and ranges from 0.03 to $0.07 . .^{29,30}$ We have then

$$
\frac{h_{\mathrm{D}}}{g t^{2}}=\frac{4 \epsilon_{d}}{\eta_{b}},
$$

independent of time. This ratio is computed in Table I. It is of order unity and decreases slightly with $Z_{2}$, which indicates that fuel contamination due to diffusion is as important as contamination due to turbulent mixing.

Finally, we can derive straightforward results in the $\operatorname{limit} Z_{2} \rightarrow \infty$. The Lewis number, given by Eq. (74), can be taken as Le $\approx 23 Z_{2}$. Since it is large, we can assume that the hydrodynamic motion is uncoupled from the mass diffusion problem; hence, we take the results derived in Subsection III B for the position of the ablated border and mass ablation, and we assume $\theta_{b} \approx 1$. Consequently, we can derive

$$
\frac{m_{l f}}{\rho_{0} \sqrt{\kappa_{0} t}} \approx \frac{0.12}{\sqrt{Z_{2}}},
$$




$$
\epsilon_{d} \approx \frac{0.29}{\sqrt{Z_{2}}}
$$

It gives $100 \times m / m_{l f} \approx 2.36 \%$ and $h_{\mathrm{D}} / g t^{2} \approx 0.72$, independent of $Z_{2}$.

\section{CONCLUSIONS}

The effect of the liner material on mass ablation, energy and magnetic flux losses, and liner-fuel mass diffusion has been studied in a MagLIF fusion-like plasma. The selfsimilar evolution of a hot magnetized fuel plasma in contact with a cold dense unmagnetized liner plasma has been thoroughly described.

In the first part of the paper, mass diffusion at the linerfuel interface (ablated border) has been neglected. The problem is governed by heat conduction, and the fuel energy is lost in heating up the ablated liner material. The magnetic field in the fuel is convected by the Nernst velocity towards the liner. It penetrates into the ablated material and diffuses in a thin layer close to the ablation front. The ablated border is a contact discontinuity, and the plasma density at the liner side is greater than at the fuel side. This configuration is Rayleigh-Taylor unstable as the ablated border penetrates into the hot spot while being decelerated by the light fuel. The magnetic field diffuses in a thin layer placed at the fuel side of the ablated border and decreases its value to compensate the variation of the Nernst velocity with the atomic number $Z$. The width of this diffusive layer scales with the inverse of the magnetic Lewis number $\mathrm{Le}_{\mathrm{m}}$, being therefore thinner than the diffusive layer at the ablation front, which scales with the inverse of the square root of $\mathrm{Le}_{\mathrm{m}}$.

For moderate and small magnetization levels, both thermal energy and magnetic flux losses in the fuel decrease with the liner atomic number $Z_{2}$, while mass ablation and magnetic flux losses in the hot spot, composed of the ablated liner and fuel regions, present a maximum for $Z_{2}=4$ and $Z_{2}$ $=12$, respectively. An asymptotic analysis performed in the large $Z_{2}$ limit shows that the four quantities scale as $1 / \sqrt{Z_{2}}$.

In the second part of this paper, mass diffusion is taken into account, but only the unmagnetized limit has been studied. The problem is governed by the Lewis number Le, which only depends on the fuel and liner atomic numbers, and is an increasing function of the latter. In a MagLIF context, it is typically large; hence, heat conduction governs the evolution of the problem, with mass diffusion being confined within a thin layer placed at the ablated border. The width of this layer scales with the inverse of the square root of Le. Among the mechanisms giving rise to mass diffusion in a plasma, classical diffusion due to concentration gradients is predominant for moderate $Z_{2}$, while baro-diffusion becomes the most important for large $Z_{2}$. Since the diffusion layer is thin and the temperature does not change notably inside, thermo-diffusion results in a minor effect. The temperature and velocity profiles are similar to the solution without mass diffusion, and the mass diffusion problem can therefore be solved a posteriori.

The amount of liner material that diffuses into the fuel scales as $1 / \sqrt{Z_{2}}$. This mass represents a small percentage of the total liner mass ablated into the hot spot. The percentage decreases with $Z_{2}$ but attains a minimum of $2.36 \%$ that cannot be further reduced. Straightforward estimations suggest that the liner material diffused pollutes the fuel in a layer that is comparable to the turbulent mixing layer induced by the Rayleigh-Taylor instability that can develop at the ablated border. This indicates that liner fuel mixing by microscopic motion (diffusion) may therefore be as important as mixing due to macroscopic motion (hydrodynamic instabilities leading to turbulence).

\section{ACKNOWLEDGMENTS}

This research was supported by the Spanish Ministerio de Economía y Competitividad, Project No. ENE201454960R and by the Spanish Ministerio de Educación, Cultura y Deporte under the national research program FPU, Grant No. FPU 14/04879. We thank Professor Riccardo Betti from Laboratory for Laser Energetics, University of Rochester, for fruitful discussion.

\section{APPENDIX: REVIEW OF TRANSPORT COEFFICIENTS}

Here, we summarize the theory already developed in Refs. 17-20 leading to the derivation of the electron and ion heat fluxes, $q_{e}$ and $q_{i}$, respectively, and the drift velocity $u_{1}$ in a two ion species plasma.

We first introduce the electron-electron collision frequency

$$
\nu_{e e}=\frac{4 \sqrt{2 \pi} n_{e} e^{4} \log \Lambda_{e e}}{3 m_{e}^{1 / 2} T_{e}^{3 / 2}}
$$

the electron-ion collision frequency

$$
\nu_{e m}=\frac{4 \sqrt{2 \pi} n_{m} Z_{m}^{2} e^{4} \log \Lambda_{e m}}{3 m_{e}^{1 / 2} T_{e}^{3 / 2}},
$$

and the ion-ion collision frequency

$$
\nu_{m n}=\frac{4 \sqrt{\pi} n_{n} Z_{m}^{2} Z_{n}^{2} e^{4} \log \Lambda_{m n}}{3 m_{m}^{1 / 2} T^{3 / 2}} .
$$

Throughout the Appendix, the subscripts $m$ and $n$ only refer to ion species. We assume the same value for the Coulomb logarithms $\log \Lambda_{e e}=\log \Lambda_{e m}=\log \Lambda_{m n}$.

The electron heat flux $q_{e}$ for a plasma with multiple ion species was independently obtained in Refs. 17 and 18 using a different variational principle, but yielding the same result

$$
q_{e}=-\frac{\gamma_{0} p_{e}}{m_{e}\left(\nu_{e 1}+\nu_{e 2}\right)} \nabla T_{e}
$$

The coefficient $\gamma_{0}$ is a function of the effective ion charge $Z_{\text {eff }}$, defined as

$$
Z_{\text {eff }} \equiv \frac{\nu_{\mathrm{e} 1}+\nu_{\mathrm{e} 2}}{\nu_{e e}}=\frac{Z_{1}^{2} n_{1}+Z_{2}^{2} n_{2}}{Z_{1} n_{1}+Z_{2} n_{2}} \geq 1,
$$

and reads 


$$
\gamma_{0}\left(Z_{\mathrm{eff}}\right)=\frac{25 Z_{\mathrm{eff}}\left(5,299,888 Z_{\mathrm{eff}}^{3}+21,559,755 \sqrt{2} Z_{\mathrm{eff}}^{2}+17,831,746 Z_{\mathrm{eff}}+1,272,672 \sqrt{2}\right)}{4\left(2,447,104 Z_{\mathrm{eff}}^{4}+17,445,571 \sqrt{2} Z_{\mathrm{eff}}^{3}+57,670,090 Z_{\mathrm{eff}}^{2}+16,033,384 \sqrt{2} Z_{\mathrm{eff}}+2,013,696\right)} .
$$

The electron heat flux can be written into a Spitzer form as

$$
q_{e}=-\bar{K}_{e}\left(Z_{\mathrm{eff}}\right) T^{5 / 2} \frac{\partial T}{\partial x},
$$

with the Spitzer coefficient for the electron heat flux defined as

$$
\bar{K}_{e}\left(Z_{\text {eff }}\right) \equiv \frac{3 \gamma_{0}\left(Z_{\text {eff }}\right)}{4 \sqrt{2 \pi m_{e}} e^{4} \log \Lambda_{e e} Z_{\text {eff }}} .
$$

Note that this expression is the same as its counterpart for a single ion species plasma, Eq. (10), substituting $Z$ for $Z_{\text {eff }}$.

Expressions for the ion drift velocities $u_{m}$ and the ion heat flux $q_{i}$ have been derived in Ref. 19 for an unmagnetized plasma with multiple ion species, and particularized for a two ion species plasma in Ref. 20. As stated in these references, $u_{m}$ and $q_{i}$ are identified as thermodynamic fluxes that can be related to their conjugate thermodynamic forces $d_{m}$, $\partial \log T_{i} / \partial x$, respectively, through a symmetric transport matrix

$$
\left(\begin{array}{c}
u_{1} \\
u_{2} \\
q_{i} / p_{i}
\end{array}\right)=-\left(\begin{array}{ccc}
\Delta_{11} & \Delta_{12} & D_{T 1} \\
\Delta_{21} & \Delta_{22} & D_{T 2} \\
D_{T 1} & D_{T 2} & \kappa_{i} / n_{i}
\end{array}\right)\left(\begin{array}{c}
d_{1} \\
d_{2} \\
\frac{\partial \log T_{i}}{\partial x}
\end{array}\right)
$$

with $\Delta_{12}=\Delta_{21}$ being the generalized diffusion coefficients, $k_{i}$ being the ion heat conduction coefficient, and $D_{T m}$ being the thermo-diffusion coefficients (the relation between $u_{m}$ and $\partial \log T_{i} / \partial x$ is often referred to as the Ludwig-Soret effect and reciprocal relation between $q_{i}$ and $d_{m}$ is referred to as the Dufour effect).

The thermodynamic force $d_{m}$ stands for the imbalance between the fluid inertial force on the ion species $m$ ( $\rho_{m} D v / D t$, with $D / D t$ being the substantial derivative) and the kinetic forces acting on it. The diffusion fluxes arise to relax this imbalance, the origin of which lies in the collision between ion species. It can be written as

$d_{m} \equiv \frac{1}{p_{i}}\left\{\frac{\partial p_{m}}{\partial x}-Z_{m} e n_{m} E-F_{m e}-\frac{\rho_{m}}{\rho}\left(\frac{\partial p_{i}}{\partial x}-e n_{e} E-\sum_{m} F_{m e}\right)\right\}$

with $E$ being the electric field and $F_{e m}=-F_{m e}$ $=-\left(\beta_{0} n_{e} \nu_{e m} / \sum_{m} \nu_{e m}\right) \partial T_{e} / \partial x$ being the electron collisional friction force with the ion species $m$, such that $\sum_{m} F_{e m}=-\beta_{0} n_{e} \partial T_{e} / \partial x$. The coefficient $\beta_{0}$ is a function of the effective ion charge, $\beta_{0}\left(Z_{\text {eff }}\right)=30 Z_{\text {eff }}\left(11 Z_{\text {eff }}+15 \sqrt{2}\right) /$ $\left(217 Z_{\text {eff }}^{2}+604 \sqrt{2} Z_{\text {eff }}+288\right)$.
The electric field can be obtained from the electron momentum equation which, after neglecting electrons inertia and making use of the ambipolarity condition, reads

$$
e n_{e} E=-\frac{\partial p_{e}}{\partial x}-\beta_{0} n_{e} \frac{\partial T_{e}}{\partial x} .
$$

Inserting these relations into Eq. (A10), and taking into account that $p_{m}=x_{m} p_{i}$, we can obtain more convenient expressions for $d_{m}$

$$
\begin{aligned}
d_{m}= & \frac{\partial x_{m}}{\partial x}+\left(x_{m}-\frac{\rho_{m}}{\rho}\right) \frac{\partial \log p_{i}}{\partial x} \\
& +\left(\frac{\rho_{m}}{\rho}-\frac{Z_{m} n_{m}}{n_{e}}\right) \frac{n_{e}}{n} \frac{e E}{T_{i}} \\
& +\left(y-\frac{Z_{m}^{2} n_{m}}{Z_{1}^{2} n_{1}+Z_{2}^{2} n_{2}}\right) \frac{n_{e}}{n} \frac{\beta_{0}}{T_{i}} \frac{\partial T_{e}}{\partial x}
\end{aligned}
$$

or, equivalently, using Eq. (A11)

$$
\begin{aligned}
d_{m}= & \frac{\partial x_{m}}{\partial x}+\left(x_{m}-\frac{\rho_{m}}{\rho}\right) \frac{\partial \log p_{i}}{\partial x} \\
& +\left(\frac{Z_{m} n_{m}}{n_{e}}-\frac{\rho_{m}}{\rho}\right) \frac{1}{p_{i}} \frac{\partial p_{e}}{\partial x} \\
& +\left(\frac{Z_{m} n_{m}}{n_{e}}-\frac{Z_{m}^{2} n_{m}}{Z_{1}^{2} n_{1}+Z_{2}^{2} n_{2}}\right) \frac{n_{e}}{n} \frac{\beta_{0}}{T_{i}} \frac{\partial T_{e}}{\partial x} .
\end{aligned}
$$

Notice that, although the original expression for $d_{m}$, Eq. (A10), is the same as Eq. (20) in Ref. 19, the more convenient expression (A13) differs from its counterpart in the same reference, Eq. (25) therein, in the coefficient $n_{e} / n$ multiplying the temperature derivative. We suspect that this factor has been dropped by mistake, since Eq. (A13) agrees with the definition of the same thermodynamic force in Eq. (125) in Ref. 18.

The system (A9) can be reduced noticing that $\sum_{m} d_{m}=0$, yielding

$$
\left(\begin{array}{c}
u_{1} \\
q_{i} / p_{i}
\end{array}\right)=-\left(\begin{array}{cc}
\Delta_{11}^{\prime} & D_{T 1} \\
D_{T 1}^{\prime} & \kappa_{i} / n_{i}
\end{array}\right)\left(\begin{array}{c}
d_{1} \\
\frac{\partial \log T_{i}}{\partial x}
\end{array}\right),
$$

where $\Delta_{11}^{\prime} \equiv \Delta_{11}-\Delta_{12}, D_{T 1}^{\prime} \equiv D_{T 1}-D_{T 2}$. As done in Ref. 20 , it is convenient to normalize the coefficients in Eq. (A14) as

$$
\begin{aligned}
& \Delta_{11}^{\prime}=\frac{2 T}{m_{1} \nu_{11}} \hat{\Delta}_{11}^{\prime}, \quad D_{T 1}=\frac{2 T}{m_{1} \nu_{11}} \hat{D}_{T 1}, \\
& D_{T 1}^{\prime}=\frac{2 T}{m_{1} \nu_{11}} \hat{D}_{T 1}^{\prime}, \quad \kappa_{i}=\frac{2 n_{1} T}{m_{1} \nu_{11}} \hat{\kappa}_{i} .
\end{aligned}
$$


The fuel drift velocity can then be written as

$$
u_{1}=-\frac{2 T}{m_{1} \nu_{11}} \hat{\Delta}_{11}^{\prime}\left(d_{1}+\frac{\hat{D}_{T 1}^{\prime}}{\hat{\Delta}_{11}^{\prime}} \frac{\partial \log T_{i}}{\partial x}\right)
$$

and we use this relation to express the ion heat flux in a more convenient way

$$
q_{i}=-\frac{2 p_{i} T}{m_{1} \nu_{11}}\left(x_{1} \hat{\kappa}_{i}-\frac{\hat{D}_{T 1}}{\hat{\Delta}_{11}^{\prime}} \hat{D}_{T 1}^{\prime}\right) \frac{\partial \log T_{i}}{\partial x}+\frac{\hat{D}_{T 1}^{\prime}}{\hat{\Delta}_{11}^{\prime} x_{1}} p_{1} u_{1}
$$

which can therefore be written as a Spitzer term plus the contribution due to mass diffusion

$$
q_{i}=-\bar{K}_{i} T^{5 / 2} \frac{\partial T}{\partial x}+\frac{\hat{D}_{T 1}^{\prime}}{\hat{\Delta}_{11}^{\prime} x_{1}} p_{1} u_{1} .
$$

The Spitzer coefficient for the ion heat flux is

$$
\bar{K}_{i}(v) \equiv \frac{3}{2 \sqrt{\pi} Z_{1}^{4} e^{4} \log \Lambda_{11} \sqrt{m_{1}}}\left(\hat{\kappa}_{i}-\frac{\hat{D}_{T 1} \hat{D}_{T 1}^{\prime}}{x_{1} \hat{\Delta}_{11}^{\prime}}\right) .
$$

A straightforward estimation of the electron and ion conduction coefficients gives $\bar{K}_{i} / \bar{K}_{e} \sim \sqrt{m_{e} / m_{p}} \ll 1$, which implies that $q_{i}$ is smaller than $q_{e}$, as happens in an unmagnetized single ion species plasma. Nevertheless, ion heat flux is retained in this analysis.

In Eq. (A16), every mechanism contributing to ion diffusion in an unmagnetized plasma can be identified. The first term of $d_{1}$, Eq. (A12), represents diffusion due to concentration gradients, the second one stands for ion baro-diffusion, the third one is the electro-diffusion, and the fourth one represents thermo-diffusion due to electron temperature gradients. The Ludwig-Soret effect in Eq. (A16) stands for thermo-diffusion driven by ion temperature gradients. Noticing that the fuel number density fraction $x_{1}$ can be related to the fuel mass concentration $y$ through

$$
x_{1}=\frac{y}{y+\frac{1-y}{\mu}},
$$

and we can obtain the same baro and electro-diffusion ratios, $k_{p}$ and $k_{E}$, as derived by Kagan and Tang ${ }^{14}$ in Eqs. (26) and (33) therein, without need to specify the transport coefficients of Eq. (A14). This confirms the statement made in the same reference that the baro- and electro-diffusion ratios can be calculated uniquely by thermodynamic means, independently of the nature of collisions. Expressing $d_{m}$ by Eq. (A12) would correspond to choosing the ion mixture as the thermodynamic system; hence, only the ion pressure appears and the electric field has to be taken into account. However, using Eq. (A13) corresponds to choosing the plasma as a whole, both ion and electron pressure take place, and the electric field does not appear explicitly as the plasma is quasi-neutral. Hereinafter, Eq. (A13) will be used for $d_{1}$ since all the terms can be directly related to temperature $T$ and fuel mass concentration $y$.

The evaluation of the normalized coefficients in Eq. (A15) requires to solve a linear system of 6 equations for every value of the fuel concentration $y$, as explained in Sec. III in Ref. 20. However, analytical solutions were derived in the same reference for a mixture of ion species with disparate masses, $\mu \gg 1$. Defining $\zeta \equiv Z_{2}^{2} \log \Lambda_{12} / Z_{1}^{2} \log \Lambda_{11}$ $=Z_{2}^{2} / Z_{1}^{2}, v \equiv \nu_{12} / \nu_{11}=\left(n_{2} / n_{1}\right) \zeta=(1-y) \zeta / \mu y$, they read

$$
\begin{aligned}
\hat{\Delta}_{11}^{\prime}(y)= & \frac{\mu(\zeta+v)}{\zeta(\zeta+v \mu)} \frac{217 \sqrt{2} v^{2}+1.208 v+288 \sqrt{2}}{16\left(16 v^{2}+61 \sqrt{2} v+72\right)}, \\
\hat{D}_{T 1}(y)= & \frac{v \mu}{\zeta+v \mu} \frac{15(11 \sqrt{2} v+30)}{8\left(16 v^{2}+61 \sqrt{2} v+72\right)}, \\
\hat{D}_{T 1}^{\prime}(y)= & \frac{15(11 \sqrt{2} v+30)}{8\left(16 v^{2}+61 \sqrt{2} v+72\right)}, \\
\hat{\kappa}_{i}(y)= & \frac{25(26 \sqrt{2} v+45)}{8\left(16 v^{2}+61 \sqrt{2} v+72\right)} \\
& +\frac{125 v(35 \sqrt{2}+9 \sqrt{\mu} v)}{4 \zeta^{2}\left(5.250+1.375 \sqrt{2 \mu} v+144 \mu v^{2}\right)} .
\end{aligned}
$$

In this paper, we use the evaluation of the coefficients for any $\mu$ rather than the analytical expressions derived for large $\mu$, with the exception of the boundary layer model in Subsection V A. Finally, we solved the linear system in the particular case $y=0$ to obtain $\hat{\Delta}_{11}^{\prime}(y=0)$ for any $\mu$, required in the definition of the Lewis number, Eq. (74). It gives $\hat{\Delta}_{11}^{\prime}(0)=P_{\mu} / \zeta$, with

$$
P_{\mu}=\sqrt{\frac{\mu+1}{\mu}} \frac{434 \mu^{6}+3,912 \mu^{5}+17,071 \mu^{4}+33,152 \mu^{3}+46,764 \mu^{2}+38,080 \mu+21,000}{16 \sqrt{2}\left(16 \mu^{6}+192 \mu^{5}+1,064 \mu^{4}+3,136 \mu^{3}+5,058 \mu^{2}+4,760 \mu+2,625\right)} .
$$

${ }^{1}$ S. A. Slutz, M. C. Herrmann, R. A. Vesey, A. B. Sefkow, D. B. Sinars, D. C. Rovang, K. J. Peterson, and M. E. Cuneo, Phys. Plasmas 17, 056303 (2010).

${ }^{2}$ M. R. Gomez, S. A. Slutz, A. B. Sefkow, D. B. Sinars, K. D. Hahn, S. B. Hansen, E. C. Harding, P. F. Knapp, P. F. Schmit, C. A. Jennings et al., Phys. Rev. Lett. 113, 155003 (2014).

${ }^{3}$ R. Landshoff, Phys. Rev. 76, 904 (1949).
${ }^{4}$ M. M. Basko, A. J. Kemp, and J. Meyer-ter Vehn, Nucl. Fusion 40, 59 (2000). ${ }^{5}$ J. R. Davies, D. H. Barnak, R. Betti, E. M. Campbell, P.-Y. Chang, A. B. Sefkow, K. J. Peterson, D. B. Sinars, and M. R. Weis, Phys. Plasmas 24, 062701 (2017).

${ }^{6}$ D. H. Barnak, J. R. Davies, R. Betti, M. J. Bonino, E. M. Campbell, V. Y. Glebov, D. R. Harding, J. P. Knauer, S. P. Regan, A. B. Sefkow et al., Phys. Plasmas 24, 056310 (2017). 
${ }^{7}$ A. L. Velikovich, J. L. Giuliani, and S. T. Zalesak, Phys. Plasmas 22, 042702 (2015)

${ }^{8}$ F. García-Rubio and J. Sanz, Phys. Plasmas 24, 072710 (2017).

${ }^{9}$ S. A. Slutz and R. A. Vesey, Phys. Rev. Lett. 108, 025003 (2012).

${ }^{10}$ F. García-Rubio and J. Sanz, Phys. Plasmas 25, 042114 (2018).

${ }^{11}$ D. T. Casey, J. A. Frenje, M. G. Johnson, M. J. E. Manuel, H. G. Rinderknecht, N. Sinenian, F. H. Séguin, C. K. Li, R. D. Petrasso, P B. Radha, J. A. Delettrez, V. Y. Glebov, D. D. Meyerhofer, T. C. Sangster, D. P. McNabb, P. A. Amendt, R. N. Boyd, J. R. Rygg, H W. Herrmann, Y. H. Kim, and A. D. Bacher, Phys. Rev. Lett. 108, 075002 (2012).

${ }^{12}$ P. Amendt, O. L. Landen, H. F. Robey, C. K. Li, and R. D. Petrasso, Phys. Rev. Lett. 105, 115005 (2010)

${ }^{13}$ G. Kagan and X. Z. Tang, Phys. Plasmas 19, 082709 (2012).

${ }^{14}$ G. Kagan and X. Z. Tang, Phys. Plasmas 21, 022708 (2014).

${ }^{15}$ L. D. Landau and E. M. Lifshitz, Fluid Mechanics (Pergamon, Oxford 1959), Vol. 6.

${ }^{16}$ G. Kagan and X. Z. Tang, Phys. Lett. A 378, 1531 (2014).

${ }^{17}$ A. N. Simakov and K. Molvig, Phys. Plasmas 21, 024503 (2014).
${ }^{18}$ K. Molvig, A. N. Simakov, and E. L. Vold, Phys. Plasmas 21, 092709 (2014).

${ }^{19}$ A. N. Simakov and K. Molvig, Phys. Plasmas 23, 032115 (2016).

${ }^{20}$ A. N. Simakov and K. Molvig, Phys. Plasmas 23, 032116 (2016).

${ }^{21} \mathrm{P}$. Helander and D. Sigmar, Collisional Transport in Magnetized Plasmas (Cambridge University Press, 2005), Vol. 4.

${ }^{22}$ S. I. Braginskii, in Reviews of Plasma Physics, edited by M. A. Leontovich (Consultants Bureau, New York, 1965), Vol. 1, p. 205.

${ }^{23}$ R. Betti, M. Umansky, V. Lobatchev, V. N. Goncharov, and R. L. McCrory, Phys. Plasmas 8, 5257 (2001).

${ }^{24}$ J. Sanz and R. Betti, Phys. Plasmas 12, 042704 (2005).

${ }^{25}$ Y. B. Zel'dovich and Y. P. Raizer, Physics of Shock Waves and HighTemperature Hydrodynamic Phenomena (Dover Publ., 2002).

${ }^{26}$ M. Van Dyke, NASA STI/Recon Technical Report A 75, 1975.

${ }^{27}$ F. M. White, Heat and Mass Transfer (Addison-Wesley, 1988).

${ }^{28}$ K. Molvig, E. L. Vold, E. S. Dodd, and S. C. Wilks, Phys. Rev. Lett. 113, 145001 (2014).

${ }^{29}$ D. L. Youngs, Physica D 12, 32 (1984).

${ }^{30}$ J. Sanz, R. Betti, R. Ramis, and J. Ramírez, Plasma Phys. Controlled Fusion 46, B367 (2004). 


\section{Magnetic flux conservation in an imploding plasma}

\subsection{General overview}

In this article, a theoretical analysis of axially magnetized subsonic plasma implosions in cylindrical geometry, schematized in Fig 6.1.1, is performed. In this problem, the physics of a cooling wave propagating in a hot magnetized plasma, studied in the article in Chapter 3, appears together with the implosion dynamics. From a mathematical point of view, the main difference with the problem analyzed in Chapter 3 is the absence of a constant reference value for temperature and magnetic field, since they increase in time.

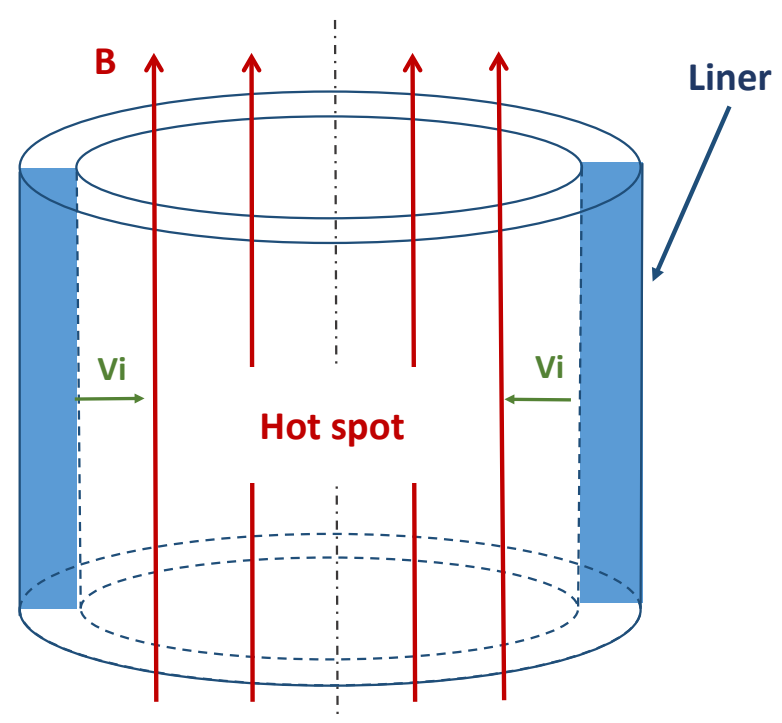

Figure 6.1.1: Scheme of a magnetized cylindrical implosion.

The motivation to perform this study comes from the identification of the Nernst term as a major mechanism degrading the performance of magnetized liner inertial fusion (MagLIF) implosions. As reported in the simulations in the seminal work by Slutz and collaborators [84], approximately $70 \%$ of the initial magnetic flux in the 
fuel is lost when the Nernst term is included, as compared to about $25 \%$ when the Nernst term is ignored. The inclusion of the Nernst term results in the decrease of the fusion yield by about $70 \%$. The hot spot model of magnetized implosions developed in this article aims at understanding the detrimental Nernst term as well as unveiling the way to reduce its effect.

A particular feature of MagLIF implosions is that the fuel is preheated before compression $[34,79,8]$. The implosion velocity is therefore subsonic at all stages. This article is based on the hot spot models of subsonic unmagnetized implosions in spherical geometry in Refs. [18, 13, 73, 74, 33], which were developed to study the deceleration phase of inertial confinement fusion (ICF) capsules. Regarding magnetized implosions, some self-similar solutions of a magnetized plasma compressed by a solid liner in cylindrical geometry have been derived thirty years ago in Refs. $[27,28]$. Both subsonic and supersonic regimes were separately analyzed. The authors proved that a cumulative compression of axial magnetic field is feasible in both regimes. In a more recent paper, Ref. [70], the physics of magnetic flux confinement and compression by a liner driven by magnetic pressure is studied analytically and numerically with applications to Z-pinch implosions. In Ref. [40], the study is generalized by addressing the case of a two components magnetic field. Neither of the last two articles took into account thermoelectric effects in their analysis.

The novelty of the magnetized implosion analysis performed in this article is that it takes into account two key features relevant for MagLIF implosions that have been disregarded in previous models: the thermoelectric Nernst effect and mass ablation. In this sense, it can be seen as an extension of the aforementioned references of ICF hot spot models where the evolution of the magnetic field coupled to the plasma dynamics has been included. The final aim is to provide scaling laws for ion density $n$, temperature $T$ and magnetic field $B$ as a function of the hot spot radius. In order to set some previous references for further comparison, it should be noticed that in an ideal adiabatic compression with adiabatic index $\gamma=5 / 3$, the previous quantities evolve as $n \sim R^{-2}, T \sim R^{-2(\gamma-1)} \sim R^{-4 / 3}, B \sim R^{-2}$, and the magnetic flux $\Phi$ is totally conserved.

\subsection{Statement of the problem}

In this article, the evolution of a cylindrical column of deuterium plasma (hot spot) surrounded by an imploding shell of cold high-density plasma (liner), made of the same material for simplicity, is considered. The problem is axisymmetric, and the independent variables are time $t$ and radial distance $r$. Only the hot spot region, enclosed by $0 \leq r \leq R(t)$, is analyzed. The implosion velocity, $V_{i}=\mathrm{d} R(t) / \mathrm{d} t$, is assumed to be constant and set by the conditions during the acceleration phase. The hot spot is fully ionized and the Braginskii's expressions and notations are used for the transport coefficients [20]. The value of the adiabatic index is $\gamma=5 / 3$. The 
problem is studied in the double limit small Mach number and large thermal-tomagnetic pressure ratio $\beta$.

The qualitative description of the problem goes as follows. The liner compresses the hot spot, increasing its thermal energy by $p \mathrm{~d} V$ work, with $p$ being the thermal pressure and $V$ the hot spot volume. The implosion is subsonic, and the thermal pressure is homogeneous and only dependent on time. Since the liner is much colder than the hot spot, its thermal conductivity is low and the heat flux is negligible. The hot spot behaves therefore like a closed system that is adiabatically heated, and $p V^{\gamma}\left(\sim p R^{2 \gamma}\right)$ is kept constant during the implosion. However, the hot spot is not a closed system, since its mass increases in time. As suggested in the hot spot model in Ref. [18], the heat flux leaving the hot spot is deposited onto the liner's inner surface, causing it to ablate. The ablated material enters the hot spot producing extra $p \mathrm{~d} V$ work. Consequently, the heat conduction losses are not real energy losses since they are recycled back into the hot spot by the ablated liner material. The interface between the hot spot and the liner, $r=R(t)$, represents an ablation front. Apart from the implosion velocity $V_{i}$, another velocity appears naturally based on the ablation rate, denoted as blow-off velocity $V_{b}$. It is defined by means of the thermal conductivity $V_{b}=2(\gamma-1) \bar{K} T_{c}^{7 / 2} / 5 \gamma p$, with $T_{c}$ being the hot spot temperature at the center, and $\bar{K}$ the Spitzer constant appearing on the thermal conductivity. It is precisely due to the hot spot mass increment that the temperature does not evolve as favorably as in an ideal adiabatic compression, $T_{c} \sim R^{-4 / 3}$. The magnetic field is compressed by the liner, and its value at the center $B_{c}$ increases with time. It is also subjected to convection and diffusion. The former is originated by the plasma motion and the Nernst term. The Nernst term convects the magnetic field outwards, enhancing its diffusion in a layer close to the liner. This is the main mechanism by which the magnetic flux is lost during the implosion.

Magnetization of the hot spot is intended to reduce thermal losses and improve magnetic flux conservation. When the plasma is magnetized, thermal conductivity is reduced and, consequently, the Nernst convection velocity diminishes. Less liner material is ablated and the temperature evolution approaches the scaling law for ideal adiabatic implosions. The effect of the Nernst term is confined within the cold unmagnetized layer near the liner. The magnetic field evolves therefore frozen into the plasma in the central region of the hot spot, and magnetic flux losses are thereby reduced.

Magnetization of the plasma is characterized by the electron Hall parameter $x_{e}$, which is given by the product of the electron cyclotron frequency and the electron collision time, $x_{e}=\omega_{e} \tau_{e}$. The electron cyclotron frequency is proportional to the magnetic field, $\omega_{e} \sim B$, while the electron collision time goes as $\tau_{e} \sim T^{3 / 2} / n$, with $n$ the plasma ion density. According to Faraday-Lenz's law, the magnetic field in the hot spot is always compressed. The evolution of the magnetization depends, however, on a balance between the collision time and the cyclotron frequency. The product $B T^{3 / 2} / n$ can increase or decrease during the implosion, hence the plasma can follow a path towards higher magnetization values or get demagnetized. 


\subsubsection{Methodology}

Plasma ion density, streamwise velocity $v$, temperature and magnetic field are given by the ion continuity, total energy conservation and induction equations, Eqs. (1) (3) in the article, together with the equation of state $p=2 n T$. Energy conservation can be integrated yielding an explicit expression for velocity, Eq. (2). The first term on the right hand side of it accounts for the contribution of the implosion dynamics, while the second stands for the contribution of heat conduction. Electrothermal and Joule effects are negligible in the large $\beta$ limit. The system can therefore be reduced to ion continuity and induction equations for the unknowns temperature $T(r, t)$ and magnetic field $B(r, t)$.

As initial conditions, the hot spot and liner are assumed to be uniformly magnetized with an external magnetic field $B_{0}$. Additionally, an initial temperature profile needs to be proposed. As boundary conditions, it is considered that the liner remains colder than the plasma in the hot spot. It acts as an electric insulator where currents cannot flow, hence the magnetic field remains equal to the external field. Since the liner density is high, the ablation velocity is smaller than the implosion velocity, and the plasma velocity at the hot spot - liner interface is equal to the latter. Axisymmetry requires temperature and magnetic field to have zero derivative at $r=0$.

The independent variables are normalized as follows. The radial coordinate is rewritten as $\eta=r / R(t)$, and the implosion time $\tau=\log \left[R_{0} / R(t)\right]$ is introduced. Temperature and magnetic field profiles are normalized with their value at the center, $\theta(\eta, \tau)=\left[T / T_{c}(\tau)\right]^{5 / 2}$ and $\phi(\eta, \tau)=B / B_{c}(\tau)$. The dimensionless governing equations become Eqs. (5) and (6). They are coupled through the dependence of the thermal conduction on the magnetization level, characterized by the electron Hall parameter $x_{e}$. They include two eigenvalues, $\lambda_{T}$ and $\lambda_{B}$. These eigenvalues describe the evolution of the variables at the center and measure how much it differs from an ideal implosion, $\lambda_{T} \equiv 2(\gamma-1)-\mathrm{d} \log T_{c} / \mathrm{d} \tau, \lambda_{B} \equiv 2-\mathrm{d} \log B_{c} / \mathrm{d} \tau$. The system is completed with the six boundary conditions, $\theta=\phi=1$ and $\partial \theta / \partial \eta=\partial \phi / \partial \eta=0$ at $\eta=0$ and $\theta=0, \phi=B_{0} / B_{c}$ at $\eta=1$. Additionally, three parameters appear: the Péclet number $\mathrm{Pe}=V_{i} / V_{b}$, the magnetic Lewis number $\mathrm{Le}_{\mathrm{m}}$ and the electron Hall parameter at the center $x_{e c}$. Since these parameters are defined with the instantaneous value of pressure, temperature and magnetic field at the center, they evolve in time. Their temporal evolution can be related to $\lambda_{T}, \lambda_{B}$, as given by Eqs. (7) - (9). They need to be solved self-consistently with the governing equations. The magnetic Lewis number and the electron Hall parameter are related to the thermalto-magnetic pressure ratio $\beta$ as $\mathrm{Le}_{\mathrm{m}} \sim \beta x_{e c}^{2}$, Eq. (4) in the article. Consequently, in order to be consistent with the $\beta \gg 1$ assumption, the analysis shall be restricted to $\mathrm{Le}_{\mathrm{m}} \gg x_{e c}^{2}$. All the information about the initial state of the problem is condensed into the initial value of these dimensionless parameters: $\mathrm{Pe}_{0}, \mathrm{Le}_{\mathrm{m} 0}$ and $x_{e c 0}$, which become the governing parameters of the system.

It may seem that normalizing the variables with their instantaneous value at the 
center leads to a more complicated reformulation of the problem, since the number of equations is increased. Effectively, in the numerical resolution of the system, an alternative normalization where temperature and magnetic field are normalized with their initial value at the center has been used, since it yields a simpler system. However, the normalization introduced in this chapter is better suited to perform an analytical study. It allows to keep track of the evolution of the shape of the profile and the growth rate of the variables separately, which is more convenient to identify different regimes and obtain analytical solutions.

An inspection of the structure of the solution for different values of $\mathrm{Pe}_{0}, \mathrm{Le}_{\mathrm{m} 0}$ and $x_{e c 0}$ has been performed. First, the unmagnetized limit has been studied, $x_{e c 0} \ll 1$, and the solution has been characterized analytically for any value of $\mathrm{Pe}_{0}, \mathrm{Le}_{\mathrm{m}}$. Then, the highly magnetized regime has been treated, and only the evolution of the variables at the center could be inferred.

\subsection{Results}

Two different asymptotic regimes have been identified. A first one denoted as "superdiffusive regime", in which the magnetization decreases during the implosion, and a second one denoted as "magnetized regime", in which the magnetization steadily increases. An implosion might fall into one of them depending on the initial value of the Péclet and magnetic Lewis numbers and electron Hall parameter.

First, the low magnetization limit, $x_{e c 0} \ll 1$, has been studied. The ion continuity, Eq. (5), gets uncoupled from induction, and can be solved independently. As shown in Refs. [18, 73, 74, 33], the solution of this type of equation converges towards a self-similar solution $\theta_{s}(\eta)$, plotted in Fig. 2(a) in the article. In this self-similar state, the product $\operatorname{Pe} \lambda_{T}$ attains a constant value, $\operatorname{Pe} \lambda_{T}=3.48$. Plugging this into the evolution equation for the Péclet number, Eq. (7), yields

$$
\frac{\mathrm{d} \log \mathrm{Pe}}{\mathrm{d} \tau}=\frac{7}{2}\left(\frac{3.48}{\mathrm{Pe}}-\frac{2}{3}\right)
$$

This equation has a stable stationary solution, $\mathrm{Pe}=\mathrm{Pe}_{s}=5.22$, independent of the initial Péclet number, which yields $\lambda_{T}=2 / 3$. This result implies that in the unmagnetized regime, the ablation process makes the flow converge to a self-similar solution in which the blow-off velocity $V_{b}$ is about five times smaller than the implosion velocity $V_{i}$. The temperature scales as $T_{c} \sim R^{-2 / 3}$, less favorably than in an ideal adiabatic implosion, since fuel energy is spent into heating up the ablated material. The hot spot mass $M_{h}=2 \pi \int_{r=0}^{r=R} n r \mathrm{~d} r$ increases as $M_{h} \sim R^{-2 / 3}$. The magnetic Lewis number can be obtained from Eq. (8). It tends to a constant value depending upon the initial conditions: $\mathrm{Le}_{\mathrm{m}}=\mathrm{Le}_{\mathrm{m} 0}\left(\mathrm{Pe}_{0} / \mathrm{Pe}_{s}\right)^{10 / 7}$.

The induction equation can be easily solved once the temperature presents the selfsimilar structure. The magnetic field profile, which starts from a homogeneous initial 
condition, tends to a stationary self-similar solution $\phi_{s}\left(\eta, \mathrm{Le}_{\mathrm{m}}\right)$, plotted in Figs. 2(c) without Nernst and 2(d) taking Nernst into account, for different magnetic Lewis numbers. When the Nernst term is not taken into account, the magnetic field is convected by the plasma motion towards the center of the hot spot, until a steep gradient develops and diffusion becomes important. Only a small central part of the hot spot remains magnetized. When the Nernst term is taken into account, the magnetic field is convected towards the liner and accumulates in a thin layer close to it, where it is dissipated, as similarly occurs in the problem analyzed in Chapter 3. The eigenvalue $\lambda_{B}$ attains a constant value dependent upon the magnetic Lewis number, $\lambda_{B}\left(\mathrm{Le}_{\mathrm{m}}\right)$, shown in Fig. 2(b). The magnetic field increases as $B_{c} \sim R^{\lambda_{B}-2}$, while the magnetic flux $\Phi=2 \pi \int_{r=0}^{r=R} \operatorname{Brd} r$ decreases as $\Phi \sim R^{\lambda_{B}}$. It is found that there is a critical Lewis number, $\mathrm{Le}_{\mathrm{m}}=0.93$, below which diffusion is so important that the magnetic field is only compressed at the beginning, it attains a certain value $B_{c} / B_{0}$ depending upon $\mathrm{Le}_{\mathrm{m}}$, shown in Fig. 2(b), and is no longer compressed throughout the implosion. Consequently, $\lambda_{B}=2$, and the magnetic flux decreases as $\Phi \sim R^{2}$. Above this critical Lewis number, $\lambda_{B}$ decreases with $\mathrm{Le}_{\mathrm{m}}$. When the Nernst term is taken into account, this eigenvalue tends to $\lambda_{B} \rightarrow 0.16$ when $\operatorname{Le}_{\mathrm{m}} \rightarrow \infty$. This means that the Nernst term originates magnetic flux losses regardless of how conductive the hot spot is. The only way to palliate this effect is by magnetizing the hot spot, that is, increasing $x_{e c}$.

The equation governing the evolution of the electron Hall parameter, Eq. (9), written in the low magnetization limit, reads

$$
\frac{\mathrm{d} \log x_{e c}}{\mathrm{~d} \tau}=\frac{1}{3}-\lambda_{B}\left(\mathrm{Le}_{\mathrm{m}}\right)
$$

Consequently, when the magnetic Lewis number is such that $\lambda_{B}<1 / 3$, the electron Hall parameter decreases steadily in time, and the hot spot remains unmagnetized throughout the implosion. This takes place for $\mathrm{Le}_{\mathrm{m}}<7.10$, or, equivalently, $\mathrm{Le}_{\mathrm{m} 0} \mathrm{Pe}_{0}^{10 / 7}<75$. This unmagnetized regime represents an attractor of the problem $\left\{\mathrm{Pe}, \mathrm{Le}_{\mathrm{m}}, x_{e c}\right\} \rightarrow\left\{\mathrm{Pe}_{s}, \mathrm{Le}_{\mathrm{m} 0}\left(\mathrm{Pe}_{0} / \mathrm{Pe}_{s}\right)^{10 / 7}, 0\right\}$, and stands for the aforementioned "superdiffusive regime".

When $\operatorname{Le}_{\mathrm{m} 0} \mathrm{Pe}_{0}^{10 / 7}>75, \lambda_{B}$ is $>1 / 3$, the electron Hall parameter increases in time and the hot spot gets highly magnetized. A second regime has been identified in the large electron Hall parameter limit. In this limit, the plasma is magnetically insulated, and heat conduction is suppressed. Particularizing the ion continuity and induction equation, Eqs. (5) and (6), at the center of the hot spot yields

$$
\lambda_{T}=-\frac{\mathcal{P}_{c}}{\mathrm{Pe}} \theta^{\prime \prime}, \quad \lambda_{B}=\frac{\mathcal{P}_{c}}{\mathrm{Pe}}\left(1-\mathcal{P}_{n}\right) \theta^{\prime \prime}-\frac{\mathcal{P}_{d}}{\mathrm{PeLe}_{\mathrm{m}}} \phi^{\prime \prime}
$$

with $\theta^{\prime \prime} \equiv \partial^{2} \theta /\left.\partial \eta^{2}\right|_{\eta=0}, \phi^{\prime \prime} \equiv \partial^{2} \phi /\left.\partial \eta^{2}\right|_{\eta=0}$. Assuming that these derivatives are finite, knowing that $\mathcal{P}_{c} \sim x_{e c}^{-2}, \mathcal{P}_{n} \approx 0.083$ and $\mathcal{P}_{d} \approx 1.95$ when $x_{e c} \gg 1$, and reminding that the inequality $\mathrm{Le}_{\mathrm{m}} \gg x_{e c}^{2}$ should hold to keep a high $\beta$, it can 
be inferred that $\lambda_{T} \sim \lambda_{B} \sim 1 / \mathrm{Pe} x_{e c}^{2}$. It is assumed now that Pex $x_{e c}^{2}$ increases in time, and hence $\lambda_{T} \sim \lambda_{B} \rightarrow 0$. The validity of this assumption is checked later. Consequently, from Eqs. (7) - (9), it can be derived that $\mathrm{Pe} \sim R^{7 / 3}, \mathrm{Le}_{\mathrm{m}} \sim R^{-10 / 3}$ and $x_{e c} \sim R^{-2}$. The electron Hall parameter increases steadily in time, and so does $\operatorname{Pe} x_{e c}^{2} \sim R^{-5 / 3}$, which confirms the assumption previously done. This regime in which the magnetization increases in time corresponds to the aforementioned "magnetized regime". The plasma at the center of the hot spot undergoes an ideal adiabatic compression, since $T_{c} \sim R^{-4 / 3}, B_{c} \sim R^{-2}$, and the magnetic flux losses are therefore reduced to zero.

Whether an implosion falls into the superdiffusive or the magnetized regime depends on the initial $\mathrm{Pe}_{0}, \mathrm{Le}_{\mathrm{m} 0}$ and $x_{e c 0}$. In Fig. 4, the frontier separating the two regimes is depicted for different values of $x_{e c 0}$. When the initial magnetization is small, the frontier corresponds to the analytical formula previously derived, $\mathrm{Le}_{\mathrm{m} 0} \mathrm{Pe}_{0}^{10 / 7}=75$, and it steps back when $x_{e c 0}$ is increased. It can be seen how important it is to design the initial parameters of the implosion so as to fall into the magnetized regime and the core of the hot spot undergoes an ideal adiabatic implosion. If the design of the implosion lies in the superdiffusive regime, the hot spot will get demagnetized due to excessive magnetic flux losses.

Finally, the temporal evolution of the temperature and magnetic field profiles of an implosion reaching the magnetized regime are shown in Figs. 3(a) and 3(b). The initial conditions belong to the MagLIF parameter range: $\mathrm{Pe}_{0}=50, \mathrm{Le}_{\mathrm{m}}=100$ and $x_{e c 0}=1.5$, and the initial temperature profile has been chosen as $\theta_{0}(\eta)=$ $\cos \left(\pi \eta^{6} / 2\right)$. The temperature profile develops two distinguishable regions. A first one close to the liner, where the temperature remains low and the plasma unmagnetized. The ablated material penetrates into the hot spot, going through this first region until it reaches the magnetically insulated region at the center, where $\mathcal{P}_{c} \approx 0$ and plasma motion due to heat conduction is suppressed. More and more layers of cold material are accumulated at the outer first region, cooling it down and forming an ablation front like structure that separates the hot highly magnetized plasma from the cold less magnetized plasma.

When the magnetization increases, the Nernst term is reduced so it remains confined within the unmagnetized region close to the liner. There, the magnetic field is pushed towards the liner and dissipated in a thin layer. Deeper into the hot spot, the Nernst term becomes less effective and the advection direction is inverted. The magnetic field is convected by the plasma motion inwards and accumulates at the border of the highly magnetized region. Consequently, the magnetic field in the less magnetized region is expelled out both to the liner and to the hot plasma. The magnetic field dissipated at the liner cannot thereby be replaced and the normalized B-field in the cold region decreases. During this process, the width of the diffusion layer is maintained, but the magnetic field peak is reduced until the layer eventually vanishes and the magnetic flux losses are thereon suppressed. This stands for the mechanism by which magnetizing the hot spot reduces the magnetic flux losses. 



\title{
Magnetic flux conservation in an imploding plasma
}

\author{
F. García-Rubio* and J. Sanz \\ E.T.S.I. Aeronáutica y del Espacio, Universidad Politécnica de Madrid, Madrid 28040, Spain
}

R. Betti

Laboratory for Laser Energetics, Department of Mechanical Engineering, and Physics and Astronomy, University of Rochester, Rochester, New York 14623, USA

(Received 7 June 2017; published 8 January 2018)

\begin{abstract}
The theory of magnetic flux conservation is developed for a subsonic plasma implosion and used to describe the magnetic flux degradation in the MagLIF concept [S. A. Slutz et al., Phys. Plasmas 17, 056303 (2010)]. Depending on the initial magnetic Lewis and Péclet numbers and the electron Hall parameter, the implosion falls into either a superdiffusive regime in which the magnetization decreases or a magnetized regime in which the magnetization increases. Scaling laws for magnetic field, temperature, and magnetic flux losses in the hot spot of radius $R$ are obtained for both regimes. The Nernst velocity convects the magnetic field outwards, pushing it against the liner and enhancing the magnetic field diffusion, thereby reducing the magnetic field compression and degrading the implosion performance. However, in the magnetized regime, the core of the hot spot becomes magnetically insulated and undergoes an ideal adiabatic compression $\left(T \sim R^{-4 / 3}\right.$ compared to $T \sim R^{-2 / 3}$ without magnetic field), while the detrimental Nernst term is confined to the outer part of the hot spot. Its effect is drastically reduced, improving the magnetic flux conservation.
\end{abstract}

DOI: 10.1103/PhysRevE.97.011201

In inertial confinement fusion (ICF), fuel areal densities $\rho R>0.3 \mathrm{~g} / \mathrm{cm}^{2}$ and implosion velocities exceeding $30 \mathrm{~cm} / \mu \mathrm{s}$ are required for the onset of the ignition process [1]. Magneto-inertial fusion concepts [2,3] are based on magnetizing the fuel in order to suppress heat losses and enhance confinement of the fusion products [4], thereby relaxing implosion velocity and areal density requirements and increasing fusion yields $[5,6]$.

In 2010, Slutz et al. [7] proposed the magnetized liner inertial fusion (MagLIF) scheme, in which a pulsed power device drives the implosion of a cylindrical beryllium liner filled with fusion fuel. The target is initially magnetized with a 10-30 T magnetic field, and the fuel is preheated by a laser to temperatures $200-400 \mathrm{eV}$. The implosion velocity is of the order of 10 $\mathrm{cm} / \mu \mathrm{s}$, while the final areal density is about $0.07 \mathrm{~g} / \mathrm{cm}^{2}$. The first fully integrated experiments testing the MagLIF concept produced up to $2 \times 10^{12}$ thermonuclear deuterium-deuterium neutrons, demonstrating its viability [8]. In 2012, Slutz and Vesey [9] reported implosions simulations of targets in which a dense cryogenic deuterium-tritium layer is added on the inside surface of the metal liner. The fusion energy gains exceeded 100 , which is adequate for fusion energy applications.

A major degradation mechanism reported in Ref. [7] is the loss of magnetic flux during compression caused by the Nernst effect (magnetic field convection due to perpendicular temperature gradients), which degrades the fusion yield enhancement. Understanding the Nernst effect as well as the evolution of the magnetohydrodynamic variables is a crucial step to improve the design of this scheme. In MagLIF implosions, the Mach number Ma is typically less than unity. Subsonic unmagnetized

*Corresponding author: fernando.garcia.rubio@upm.es implosions have been analytically studied in an ICF context [10-13], whereas the Nernst term effect on magnetic flux conservation in a plasma slab in contact with a cold wall has been investigated in Refs. [14,15].

In this work, we describe a detailed analytical model of a cylindrical subsonic magnetized plasma implosion, relevant for free fall, deceleration, and stagnation phases. We consider a deuterium plasma (hot spot) surrounded by an imploding shell of cold high-density plasma (liner) made of the same material for simplicity. The independent variables are time $t$ and radial distance $r$. We analyze the hot spot region limited by the inner wall of the liner, $r=R(t)$. The implosion velocity $V_{i}=$ $-d R / d t$ is assumed to be constant and set by the conditions during the acceleration phase. We consider a fully ionized plasma fluid model with Braginskii's expressions and notation for the transport coefficients [16] and the ideal gas assumption with adiabatic index $\gamma=5 / 3$. The state of the plasma is determined by the ion particle density $n$, plasma temperature $T$, pressure $p=p_{i}+p_{e}=2 n T$, radial velocity $v$, and axial magnetic field $B$. We introduce the thermal to magnetic pressure ratio $\beta=8 \pi p_{c} / B_{c}^{2}$. Hereinafter, the subindex $c$ denotes the value of the variables at the center $r=0$ (symmetrical axis), which evolves in time, while the subindex 0 refers to the value at $t=0$. The problem is greatly simplified noticing that Ma $\ll 1$ and $\beta \gg 1$ in many typical implosions. The analysis is performed in this double limit. Momentum conservation simplifies to isobaricity $p(r, t)=p_{c}(t)$. Ion continuity, total energy conservation, and induction equations read

$$
\begin{gathered}
\frac{\partial n}{\partial t}+\frac{1}{r} \frac{\partial}{\partial r}(r v n)=0, \\
v=-\frac{r}{2 \gamma p_{c}} \frac{d p_{c}}{d t}+\frac{\gamma-1}{\gamma p_{c}} \chi_{\perp} \frac{\partial T}{\partial r},
\end{gathered}
$$




$$
\frac{\partial B}{\partial t}+\frac{1}{r} \frac{\partial}{\partial r}(r v B)=\frac{1}{r} \frac{\partial}{\partial r}(\underbrace{D_{m \perp} \frac{\partial B}{\partial r}}_{\text {Joule }}+r \underbrace{\frac{c \beta_{\wedge}^{u T}}{e n} \frac{\partial T}{\partial r}}_{\text {Nernst }}) .
$$

The coefficient $\chi_{\perp}$ accounts for electron plus ion conductivities, $D_{m \perp}=\alpha_{\perp} c^{2} / 4 \pi e^{2} n^{2}$ is the magnetic field diffusion coefficient appearing in the Joule term, and $\beta_{\wedge}^{u T}$ refers to the transport coefficient for the thermoelectric Nernst effect.

As initial conditions, we impose that the target is uniformly magnetized with an external magnetic field $B_{0}$. Additionally, an initial temperature profile needs to be proposed. As boundary conditions, we consider that during the implosion, the liner remains colder than the plasma in the hot spot: $T(R, t)=0$. The cold dense liner acts as an electric insulator where currents cannot flow, and the axial magnetic field is equal to the external field, $B(R, t)=B_{0}$. In addition, we require $v=d R / d t\left(=-V_{i}\right)$ at $r=R$. The thermal conductivity can be neglected at the inner liner surface, and the heat conduction losses are recycled back via the ablated liner material $[10,11]$ ( $r=R$ represents an ablation front). The energy conservation balance in the hot spot enclosed by the cold liner states that, although the hot spot mass increases with time, it behaves like a closed system that is adiabatically heated: $p_{c} R^{2 \gamma}$ is kept constant.

We normalize the spatial coordinate, $\eta=r / R(t)$, the implosion time, $\tau=\log \left[R_{0} / R(t)\right]$, the plasma temperature $\theta(\eta, \tau)=\left[T / T_{c}(\tau)\right]^{5 / 2}$, magnetic field $\phi(\eta, \tau)=B / B_{c}(\tau)$, and velocity $u(\eta, \tau)=v / V_{i}$. The transport coefficients can therefore be written as $\chi_{\perp}=\bar{K} T_{c}^{5 / 2} \theta \mathcal{P}_{c}, D_{m \perp}=$ $\bar{D} T_{c}^{-3 / 2} \theta^{-3 / 5} \mathcal{P}_{d}$, with $\bar{K}=8.64 \times 10^{27} \mathrm{sec}^{-1} \mathrm{~cm}^{-1} \mathrm{keV}^{-5 / 2}$ and $\bar{D}=93.2 \mathrm{~cm}^{2} \mathrm{keV}^{3 / 2} / \mathrm{sec}$ conductivity and diffusivity constants, and $c \beta_{\wedge}^{u T} / e n=\left[(\gamma-1) \chi_{\perp} / \gamma p\right] \mathcal{P}_{n} B$. The latter relation indicates that the Nernst term in Eq. (3) accounts for magnetic field convection in the heat flux direction. The terms $\mathcal{P}_{c}\left(x_{e}\right), \mathcal{P}_{n}\left(x_{e}\right)$, and $\mathcal{P}_{d}\left(x_{e}\right)$ represent the effect of magnetization on thermal conduction, Nernst effect, and magnetic diffusivity, respectively [14]. They are rational functions of the local electron Hall parameter (electron cyclotron frequency times the electron collision time) $x_{e}=\omega_{e} \tau_{e}=x_{e c} \theta \phi$ and plotted in Fig. 1:

$$
\begin{aligned}
& \mathcal{P}_{c}\left(x_{e}\right)=\frac{\gamma_{1}^{\prime} x_{e}^{2}+\gamma_{0}^{\prime}}{\gamma_{0} \Delta_{e}}+\sqrt{\frac{2 m_{e}}{m_{i}}} \frac{2 x_{i}^{2}+2.645}{\gamma_{0} \Delta_{i}}, \\
& \mathcal{P}_{n}\left(x_{e}\right)=\frac{2 \gamma}{\gamma-1} \frac{\beta_{1}^{\prime \prime} x_{e}^{2}+\beta_{0}^{\prime \prime}}{\gamma_{1}^{\prime} x_{e}^{2}+\gamma_{0}^{\prime}+\sqrt{\frac{2 m_{e}}{m_{i}}} \frac{\Delta_{e}}{\Delta_{i}}\left(2 x_{i}^{2}+2.645\right)}, \\
& \mathcal{P}_{d}\left(x_{e}\right)=\frac{1-\frac{\alpha_{1}^{\prime} x_{e}^{2}+\alpha_{0}^{\prime}}{\Delta_{e}}}{\alpha_{0}},
\end{aligned}
$$

with $\Delta_{e}=x_{e}^{4}+\delta_{1} x_{e}^{2}+\delta_{0}$ and $\Delta_{i}=x_{i}^{4}+2.70 x_{i}^{2}+0.677$, and $x_{i}=\omega_{i} \tau_{i}=\sqrt{2 m_{e} / m_{i}} x_{e}$ standing for the ion magnetization. The values for the coefficients $\gamma_{0}^{\prime}, \delta_{0}$, and so on are taken from Braginskii [16] and specified for a deuterium plasma $(Z=1, A=2)$. A blow-off velocity based on thermal conduction can be defined as $V_{b}=2(\gamma-1) \bar{K} T_{c}^{7 / 2} / 5 \gamma R p_{c}$ [10-13]. The global plasma parameters are thereby reduced to three dimensionless numbers: the Péclet number $\mathrm{Pe}=V_{i} / V_{b}$,

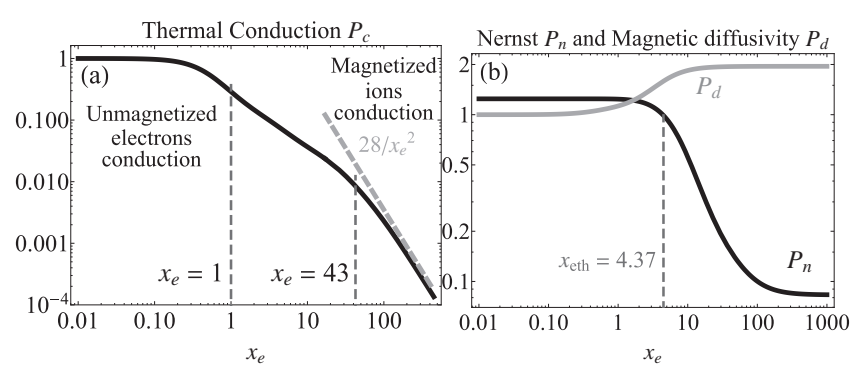

FIG. 1. Dependence of transport coefficients on the magnetization (electron Hall) parameter $x_{e}$.

the magnetic Reynolds number $\operatorname{Re}=V_{i} R / D_{c}$, with $D_{c}=$ $\bar{D} T_{c}^{-3 / 2}$, and the electron Hall parameter at the center $x_{e c}$. These numbers evolve during the implosion. For convenience, we use the magnetic Lewis number instead of the Reynolds, defined as Le $=V_{b} R / D_{c}=\mathrm{Re} / \mathrm{Pe}$. In addition, the thermal to magnetic pressure ratio $\beta$ can be related to Le, $x_{e c}$ through

$$
\mathrm{Le}=\frac{(\gamma-1) \gamma_{0}}{20 \gamma \alpha_{0}} \beta x_{e c}^{2}=0.12 \beta x_{e c}^{2}
$$

In order to be consistent with the $\beta \gg 1$ assumption, we restrict our analysis to Le $\gg x_{e c}^{2}$.

The dimensionless velocity can be written as $u=-\eta+u_{c}$, where $u_{c}=(1 / \mathrm{Pe}) \mathcal{P}_{c} \theta^{2 / 5} \partial \theta / \partial \eta$ stands for the contribution of thermal conduction. Using this expression, Eqs. (1) and (3) after normalization read

$$
\begin{gathered}
\frac{\partial \theta^{2 / 5}}{\partial \tau}-\lambda_{T} \theta^{2 / 5}=\frac{\theta^{4 / 5}}{\operatorname{Pe} \eta} \frac{\partial}{\partial \eta}\left(\eta \mathcal{P}_{c} \frac{\partial \theta}{\partial \eta}\right), \\
\frac{\partial \phi}{\partial \tau}-\lambda_{B} \phi+\frac{1}{\operatorname{Pe} \eta} \frac{\partial}{\partial \eta}\left[\eta \mathcal{P}_{c}\left(1-\mathcal{P}_{n}\right) \theta^{2 / 5} \frac{\partial \theta}{\partial \eta} \phi\right] \\
=\frac{1}{\text { PeLe } \eta} \frac{\partial}{\partial \eta}\left(\eta \mathcal{P}_{d} \theta^{-3 / 5} \frac{\partial \phi}{\partial \eta}\right),
\end{gathered}
$$

where the eigenvalues $\lambda_{T}=\left(d n_{c} / d \tau\right) / n_{c}-2=2(\gamma-1)-$ $\left(d T_{c} / d \tau\right) / T_{c}$ and $\lambda_{B}=2-\left(d B_{c} / d \tau\right) / B_{c}$, with $n_{c}=p_{c} / 2 T_{c}$, describe the evolution of the variables at the center and measure how much it differs from an ideal implosion (infinite Pe and Re: no ablation and $n_{c} \sim R^{-2}, T_{c} \sim R^{-4 / 3}, B_{c} \sim R^{-2}$ ). Two opposite effects are accounted for in the advection term in Eq. (6): convection due to $u_{c}$, in the opposite direction to the heat flux, and convection due to the Nernst term, in the same direction. For $x_{e}<x_{e \text { th }}=4.37,1-\mathcal{P}_{n}$ is negative and the latter prevails [Fig. 1(b)]. All the information about the initial state is condensed into the initial Péclet $\mathrm{Pe}_{0}$ and Lewis $\mathrm{Le}_{0}$ numbers, the initial Hall parameter $x_{e c 0}$ and the initial profiles for $\theta$ and $\phi$.

The system (5)-(6) is coupled through the magnetization effect on the thermal conductivity, $\mathcal{P}_{c}\left(x_{e}\right)$. Solving it allows to obtain the temperature and magnetic field profiles and the eigenvalues $\lambda_{T}$ and $\lambda_{B}$. From the definition of Pe, Le, and $x_{e c}$, their evolution in time can be easily described in terms of $\lambda_{T}$ 

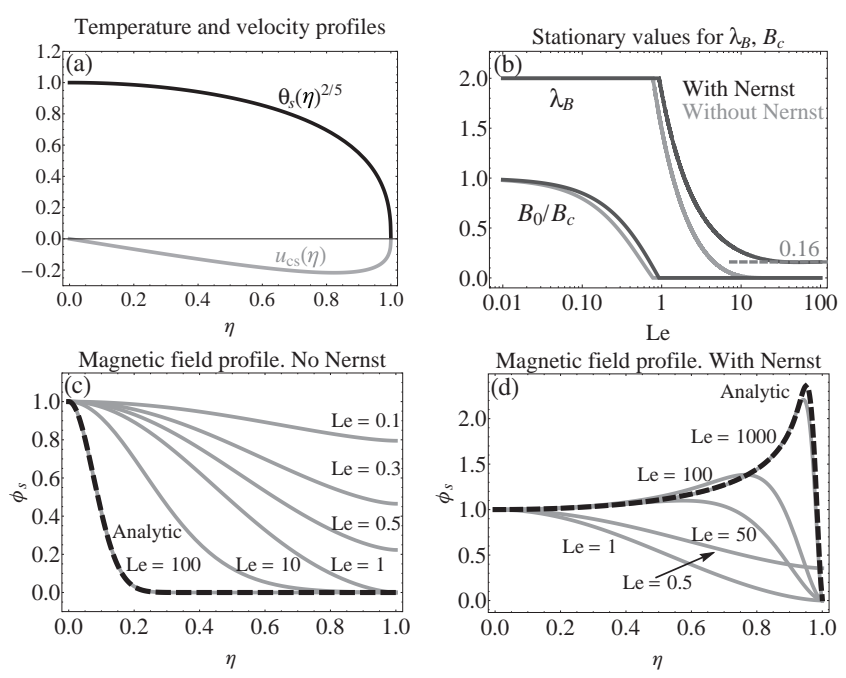

FIG. 2. Unmagnetized plasma implosion. (a) Temperature and velocity self-similar profiles. (b) Stationary values for $\lambda_{B}$ and $B_{c}$. (c, d) Magnetic field self-similar profiles.

and $\lambda_{B}$ :

$$
\begin{gathered}
\frac{d \log \mathrm{Pe}}{d \tau}=\frac{7}{2}\left(\lambda_{T}-\frac{2}{3}\right), \\
\frac{d}{d \tau}\left(\operatorname{LePe}^{10 / 7}\right)=0, \\
\frac{d \log x_{e c}}{d \tau}=2-\lambda_{B}-\frac{5}{2} \lambda_{T} .
\end{gathered}
$$

In order to gain insight into the physics of the implosion, we first consider the case where the magnetization is very low $x_{e c} \ll 1$. In this limit, $\mathcal{P}_{c}=1$, Eq. (5) is decoupled from (6) and simplifies to

$$
\frac{\partial \theta^{2 / 5}}{\partial \tau}-\lambda_{T} \theta^{2 / 5}=\frac{\theta^{4 / 5}}{\operatorname{Pe} \eta} \frac{\partial}{\partial \eta}\left(\eta \frac{\partial \theta}{\partial \eta}\right) .
$$

This equation has a stable stationary solution $\theta_{s}(\eta)$ [10-13], corresponding to a self-similar implosion [Fig. 2(a)]. In this self-similar state, $\operatorname{Pe} \lambda_{T}=3.48$, and inserting this into Eq. (7), we obtain that the Péclet number tends to a constant $\mathrm{Pe}=$ $\mathrm{Pe}_{s}=5.22$ and $\lambda_{T}=2 / 3$. Consequently the Lewis number also tends to a constant whose value depends upon the initial conditions: $\mathrm{Le}=\mathrm{Le}_{0}\left(\mathrm{Pe}_{0} / \mathrm{Pe}_{s}\right)^{10 / 7}$. The velocity self-similar profile is $u_{s}(\eta)=-\eta+\left(1 / \mathrm{Pe}_{s}\right) \theta_{s}^{2 / 5} d \theta_{s} / d \eta$. The temperature at the center evolves as $T_{c} \sim R^{-2 / 3}$, while the hot spot mass $M_{h}=2 \pi \int_{r=0}^{r=R} n r d r$ increases as $M_{h} \sim R^{-2 / 3}$. Material from the cold liner is ablated into the hot spot, cooling it down and causing the temperature increase to be less than expected for an ideal adiabatic compression of a gas $\left(T \sim R^{-4 / 3}\right)$.

In the unmagnetized limit $\left(x_{e c} \ll 1\right)$, Eq. (6) simplifies to

$$
\begin{gathered}
\frac{\partial \phi}{\partial \tau}-\lambda_{B} \phi+\frac{\delta}{\operatorname{Pe} \eta} \frac{\partial}{\partial \eta}\left(\eta \theta^{2 / 5} \frac{\partial \theta}{\partial \eta} \phi\right) \\
=\frac{1}{\text { PeLe } \eta} \frac{\partial}{\partial \eta}\left(\eta \theta^{-3 / 5} \frac{\partial \phi}{\partial \eta}\right),
\end{gathered}
$$

where the parameter $\delta=1-\mathcal{P}_{n}(0)=-0.24$ can be switched to $\delta=1$ in order to artificially turn the Nernst term off for comparison purposes. Once the temperature reaches the self-similar state, the magnetic field also tends to a stationary self-similar solution $\phi=\phi_{s}(\eta ;$ Le $)$, and $\lambda_{B}=\lambda_{B}($ Le $)$ gets independent of time. The magnetic field at the center evolves as $B_{c} \sim R^{\lambda_{B}-2}$, whereas the magnetic flux $\Phi=2 \pi \int_{r=0}^{r=R} B r d r$ decreases as $\Phi \sim R^{\lambda_{B}}$. The eigenvalue $\lambda_{B}$ and the self-similar profile $\phi_{s}$ are shown in Figs. 2(b), 2(c), and 2(d) as a function of the Lewis number.

When the Lewis number is small, the parameter $\lambda_{B}=2$, the magnetic field at the center $B_{c}$ attains a constant value, and consequently the magnetic flux decreases as $\Phi \sim R^{2}$. However, for Le $>0.93$ with Nernst and Le $>0.78$ without, $\lambda_{B}<2$ and the magnetic field at the center increases in time.

When the Lewis number is large, diffusion can be neglected in principle, and the magnetic field is convected by the plasma motion and the Nernst velocity. If the latter is not taken into account, the magnetic field is frozen into the plasma and the mass ablated into the hot spot would squeeze it inwards, steepening the B-field profile until diffusion becomes important. An asymptotic analysis in this large Lewis number and no Nernst limit shows that the self-similar profile is $\phi_{s}(\eta)=\exp \left(-\mathrm{Pe}_{s} \mathrm{Le} \eta^{2} / 6\right)$, the field is not diffused through the liner, $\lambda_{B} \rightarrow 0$ and the magnetic flux is conserved. When the Nernst term is taken into account, the Nernst convection dominates and the magnetic field is advected outwards. It penetrates into the ablated mass, and it is pushed against the liner and dissipated in a thin layer where diffusion becomes important. An asymptotic expansion for large Lewis numbers shows that the thickness of the diffusion layer is $O(1 / \sqrt{\mathrm{Le}})$, and the peak of the profile scales as $\mathrm{Le}^{1 / 5}$. The parameter $\lambda_{B} \rightarrow$ $-2 \delta / 3 \approx 0.16$ and cannot be further reduced by increasing the Lewis number, which indicates that the Nernst term plays a crucial role in degrading the magnetic flux conservation during the implosion even for a very conductive plasma.

According to Faraday-Lenz's law, the magnetic field in the hot spot is always compressed. The evolution of the magnetization depends, however, on a balance between the collision and cyclotron frequencies, Eq. (9). In the low magnetization limit $\left(\omega_{e} \tau_{e}<1, \lambda_{T}=2 / 3\right)$, the collision frequency increases more than the cyclotron frequency whenever $\lambda_{B}<1 / 3$, hence the magnetization decreases in time and the hot spot remains unmagnetized during the whole implosion. This unmagnetized regime represents an attractor of the complete system of Eqs. (5)-(6) and will be denoted as a "superdiffusive regime." For $\lambda_{B}>1 / 3$, which takes place for $\mathrm{Le}>\mathrm{Le}_{c r}=7.10$ with Nernst and Le $>3.02$ without, the collision frequency increases less than the cyclotron frequency, the hot spot gets magnetized, and the hydrodynamic profiles are consequently modified.

We consider now a strong magnetization limit, $x_{e c} \gg 1$. The plasma is magnetically insulated at the center, and the continuity and induction equations (5) and (6) give $\lambda_{T}=0$, $\lambda_{B}=0$. The central temperature and magnetic field undergo an ideal adiabatic compression: $T_{c} \sim R^{-4 / 3}, B_{c} \sim R^{-2}$. The Péclet number evolves as $\mathrm{Pe} \sim R^{7 / 3}$, and consequently Le $\sim$ $R^{-10 / 3}$. In this limit, Eq. (9) establishes that the magnetization keeps increasing in time as $x_{e c} \sim R^{-2}$, hence this regime represents another attractor, which will be denoted as a "magnetized regime." According to Eq. (4), the pressure ratio $\beta$ decreases in this limit as $\beta \sim R^{2 / 3}$. Therefore, pressure ratios 

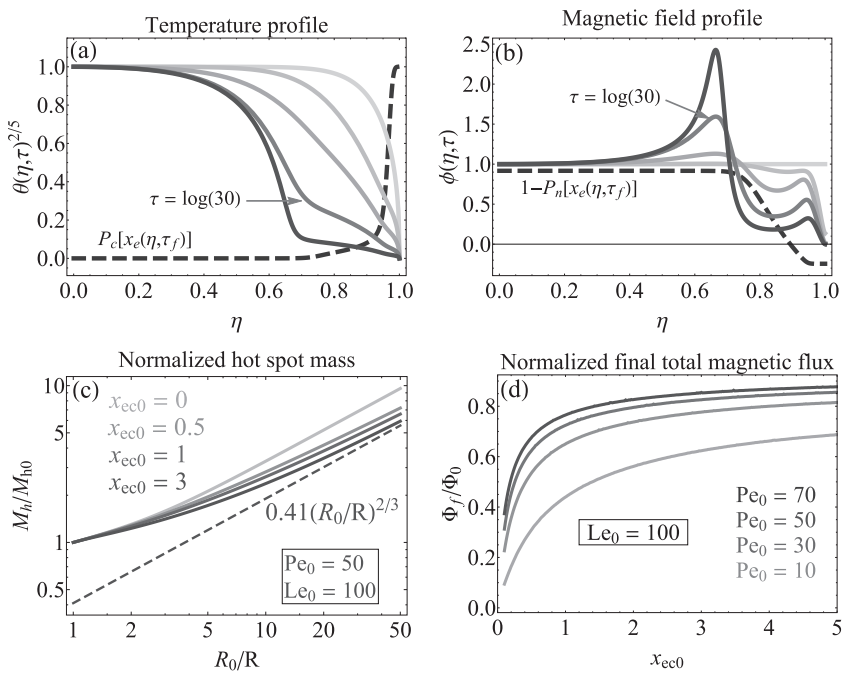

FIG. 3. (a), (b) Magnetized implosion profiles. Initial parameters: $\mathrm{Pe}_{0}=50, \mathrm{Le}_{0}=100, x_{e c 0}=1.5, \theta_{0}(\eta)=\cos \left(\pi \eta^{6} / 2\right), \phi_{0}(\eta)=1$. From light to darker gray: $\tau=0,1,2, \tau_{f}=\log (30) \approx 3.4,5$. (c) Normalized hot spot mass in implosions with the same initial parameters but different initial magnetization. (d) Normalized final magnetic flux remaining in the hot spot in implosions with different initial magnetization.

of order unity and below could be attained by the end of the implosion. Consequently, for these results to be valid in the magnetized regime for a suffciently long time, $\beta$ has to be large initially, which is typically the case in MagLIF $\left(\beta_{0} \sim\right.$ several hundred).

In Figs. 3(a) and 3(b), the temporal evolution of the temperature and magnetic field profiles of a magnetized implosion are shown. The initial state corresponds to a characteristic MagLIF parameter range: $\mathrm{Pe}_{0}=50, \mathrm{Le}_{0}=100$, and $x_{e c 0}=1.5$, which gives $\beta_{0}=361$, and we choose $\theta_{0}(\eta)=\cos \left(\pi \eta^{6} / 2\right), \phi_{0}(\eta)=$ 1 . In this case study, the magnetization increases in time and the magnetized regime is reached. The temperature profile presents two distinguishable regions. Close to the liner, where the temperature is low and the plasma is unmagnetized, cold material is ablated through the ablation front. It penetrates into the hot spot until it reaches the magnetically insulated region, where $\mathcal{P}_{c} \approx 0$ and $u_{c} \approx 0$. More and more layers of cold material are accumulated at the outer part of the hot spot, cooling it down and forming an ablation front like structure that separates the hot highly magnetized plasma from the cold less magnetized plasma.

The Nernst term is confined within the unmagnetized region. Close to the liner, where $1-\mathcal{P}_{n}<0$, the magnetic field is pushed towards it and dissipated in a thin layer. Deeper into the hot spot, the Nernst term is drastically reduced, and the advection direction is inverted: $1-\mathcal{P}_{n}>0$. The magnetic field is convected by the plasma motion inwards and accumulates at the border of the highly magnetized region. Consequently the magnetic field in the less magnetized region is expelled out both to the liner and to the hot plasma. The magnetic field dissipated at the liner cannot thereby be replaced and the normalized B-field in the cold region decreases. During this process, the width of the diffusion layer is maintained, but

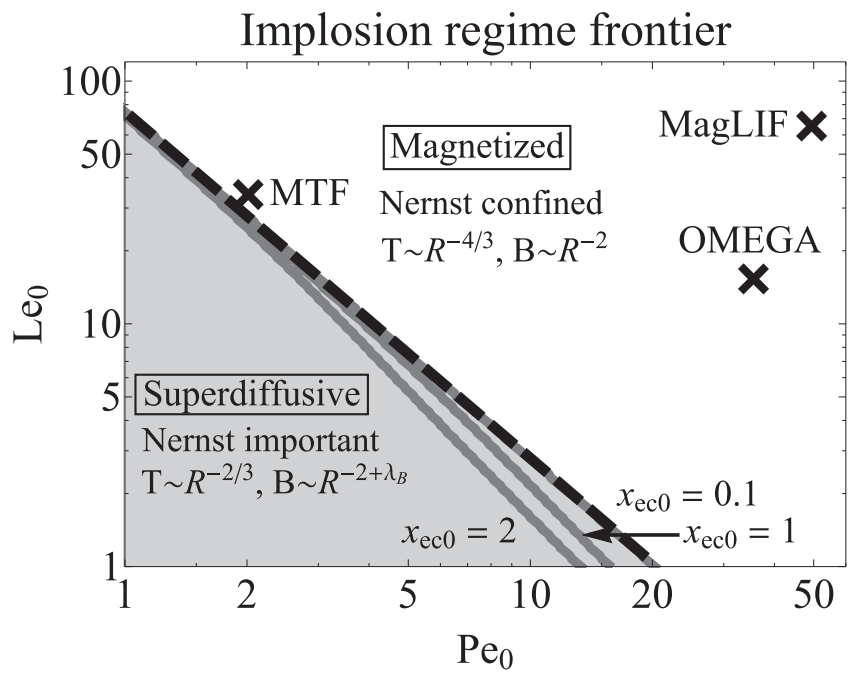

FIG. 4. Parameter frontier for superdiffusive and magnetized implosion regimes. The dashed line corresponds to $\operatorname{Le}_{0} \mathrm{Pe}_{0}^{10 / 7}=75$. The crosses indicate the position in the chart of the three schemes in Table I.

the magnetic field peak is reduced until the layer eventually vanishes and the magnetic flux losses are thereon suppressed.

The magnetization decreases mass ablation and improves magnetic flux conservation. In the magnetized regime, the hot spot mass also scales asymptotically as $M_{h} \sim R^{-2 / 3}$, but the constant of proportionality is reduced [Fig. 3(c)]. As previously commented, the diffusion layer tends to vanish in this limit, the magnetic flux losses are drastically reduced, and the magnetic flux in the hot spot reaches a constant value $\Phi_{f}$ dependent upon the initial state, shown in Fig. 3(d).

Whether an implosion falls into the superdiffusive or magnetized regime depends on the initial Péclet, Lewis, and Hall parameter. In Fig. 4 the boundary between both regimes is plotted in a $\mathrm{Pe}_{0}-\mathrm{Le}_{0}$ map for different $x_{e c 0}$. For a small initial magnetization, the border corresponds to $\operatorname{Le}_{0} \mathrm{Pe}_{0}^{10 / 7}=$ $\mathrm{Le}_{c r} \mathrm{Pe}_{s}^{10 / 7} \approx 75$, which falls back to lower values of $\mathrm{Le}_{0}$ and $\mathrm{Pe}_{0}$ when the initial magnetization is increased.

According to the results previously derived, in the design of magnetized inertial fusion implosions it is important to be placed in the "magnetized regime" part of the chart plotted in Fig. 4. This can be ensured if the implosion design point satisfies

$$
\begin{aligned}
\operatorname{Le}_{0} \mathrm{Pe}_{0}^{10 / 7}= & 386\left(\frac{T_{0}}{1 \mathrm{eV}}\right)^{3 / 7}\left(\frac{\rho_{0}}{1 \mathrm{mg} / \mathrm{cm}^{3}}\right)^{3 / 7} \\
& \times\left(\frac{R_{0}}{1 \mathrm{~cm}}\right)^{10 / 7}\left(\frac{V_{i}}{1 \mathrm{~cm} / \mu \mathrm{s}}\right)^{10 / 7}>75
\end{aligned}
$$

where $R_{0}$ and $\rho_{0}$ are the initial radius and fuel density. In Table I three different schemes have been analyzed, and their position is shown in Fig. 4. Both the pulsed power MagLIF and laser-driven magnetized liner implosions tested at Laboratory for Laser Energetics satisfy well this requirement, but the low-density, low-implosion-velocity regime explored by Lindemuth and Kirkpatrick [2] in spherical geometry may 
TABLE I. Characteristic initial values of the magnethydrodynamic variables for three different magnetized inertial fusion schemes. MagLIF corresponds to Ref. [7], OMEGA corresponds to the laserdriven magnetized liner inertial fusion studied at the LLE (OMEGA laser) in Refs. [17,18], and MTF corresponds to the magnetized target fusion regime explored in spherical geometry in Ref. [2].

\begin{tabular}{lccc}
\hline \hline & MagLIF & OMEGA & MTF \\
\hline$T_{0}(\mathrm{eV})$ & 300 & 200 & 50 \\
$\rho_{0}\left(\mathrm{mg} / \mathrm{cm}^{3}\right)$ & 3 & 2.7 & 0.005 \\
$R_{0}(\mathrm{~cm})$ & 0.2 & 0.03 & 0.5 \\
$V_{i}(\mathrm{~cm} / \mu \mathrm{s})$ & 10 & 18.8 & 1 \\
$\mathrm{Pe}_{0}$ & 53 & 37 & 2 \\
$\mathrm{Le}_{0}$ & 67 & 15 & 31 \\
$\mathrm{Le}_{0} \mathrm{Pe}_{0}^{10 / 7}$ & 20.000 & 2.500 & 80 \\
\hline \hline
\end{tabular}

need to be adjusted for cylindrical geometry since it lies close to the threshold.
To conclude, the existence of two regimes, superdiffusive and magnetized, has been proved in a hot spot model of magnetized cylindrical implosions. Scaling laws for temperature, magnetic field, mass ablation, and magnetic flux losses for every regime have been derived. The Nernst term convects the magnetic field outwards and enhances diffusion, degrading thereby the magnetic flux conservation even for a highly conductive plasma. In the magnetized regime, the core of the hot spot gets magnetically insulated, and the effect of the Nernst term is reduced since it is confined to the outer part of the hot spot close to the liner.

This research was supported by the Spanish Ministerio de Economía y Competitividad, Project No. ENE2014-54960R, the Spanish Ministerio de Educación, Cultura y Deporte under the national research program FPU, grant FPU 14/04879, and the U.S. Department of Energy, awards DE-SC0016258 and DE-AR0000568.
[1] S. Atzeni and J. Meyer-ter Vehn, The Physics of Inertial Fusion: BeamPlasma Interaction, Hydrodynamics, Hot Dense Matter, 125 (Oxford University Press on Demand, Oxford, 2004).

[2] I. R. Lindemuth and R. C. Kirkpatrick, Nucl. Fusion 23, 263 (1983).

[3] R. C. Kirkpatrick, I. R. Lindemuth, and M. S. Ward, Fusion Technol. 27, 201 (1995).

[4] M. M. Basko, A. J. Kemp, and J. Meyer-ter Vehn, Nucl. Fusion 40, 59 (2000).

[5] P. Y. Chang, G. Fiksel, M. Hohenberger, J. P. Knauer, R. Betti, F. J. Marshall, D. D. Meyerhofer, F. H. Séguin, and R. D. Petrasso, Phys. Rev Lett. 107, 035006 (2011).

[6] M. Hohenberger, P. Y. Chang, G. Fiksel, J. P. Knauer, R. Betti, F. J. Marshall, D. D. Meyerhofer, F. H. Séguin, and R. D. Petrasso, Phys. Plasmas 19, 056306 (2012).

[7] S. A. Slutz, M. C. Herrmann, R. A. Vesey, A. B. Sefkow, D. B. Sinars, D. C. Rovang, K. J. Peterson, and M. E. Cuneo, Phys. Plasmas 17, 056303 (2010).

[8] M. R. Gomez, S. A. Slutz, A. B. Sefkow, D. B. Sinars, K. D. Hahn, S. B. Hansen, E. C. Harding, P. F. Knapp, P. F. Schmit, C. A. Jennings et al., Phys. Rev. Lett. 113, 155003 (2014).
[9] S. A. Slutz and R. A. Vesey, Phys. Rev. Lett. 108, 025003 (2012).

[10] R. Betti, M. Umansky, V. Lobatchev, V. N. Goncharov, and R. L. McCrory, Phys. Plasmas 8, 5257 (2001).

[11] J. Sanz and R. Betti, Phys. Plasmas 12, 042704 (2005).

[12] J. Sanz, J. Garnier, C. Cherfils, B. Canaud, L. Masse, and M. Temporal, Phys. Plasmas 12, 112702 (2005).

[13] J. Garnier and C. Cherfils, Phys. Plasmas 12, 012704 (2005).

[14] F. García-Rubio and J. Sanz, Phys. Plasmas 24, 072710 (2017).

[15] A. L. Velikovich, J. L. Giuliani, and S. T. Zalesak, Phys. Plasmas 22, 042702 (2015).

[16] S. I. Braginskii, in Reviews of Plasma Physics, edited by M. A. Leontovich (Consultants Bureau, New York, 1965), Vol. 1, p. 205.

[17] J. R. Davies, D. H. Barnak, R. Betti, E. M. Campbell, P.-Y. Chang, A. B. Sefkow, K. J. Peterson, D. B. Sinars, and M. R. Weis, Phys. Plasmas 24, 062701 (2017).

[18] D. H. Barnak, J. R. Davies, R. Betti, M. J. Bonino, E. M. Campbell, V. Y. Glebov, D. R. Harding, J. P. Knauer, S. P. Regan, A. B. Sefkow et al., Phys. Plasmas 24, 056310 (2017). 



\section{Alpha heating enhancement in magnetized implosions}

\subsection{General Overview}

In this article, a theoretical study of the dynamics of magnetized cylindrical implosions after stagnation is performed. Special attention is paid to the alpha particles motion generated from thermonuclear reactions, which deposit some of their energy in the fuel and affect the dynamics of the hot spot expansion.

The motivation to perform this study lies in the derivation of a dynamic ignition criterion for magnetized cylindrical implosions with application to magnetized liner inertial fusion (MagLIF). The article can be seen as an extension of the analysis of the hot spot dynamics after stagnation developed in Ref. [23] for inertial confinement fusion (ICF) capsules in spherical geometry. The hot spot energy changes as a result of the imbalance between the alpha-heating and the expansion losses. In this reference, singular solutions where the alpha-heating term dominates and pressure exhibits a singularity in finite time have been identified as "ignited solutions". These solutions take place for a value of a parameter named $\chi_{\text {no } \alpha}$ larger than unity. This parameter can be seen as a normalized form of the typical Lawson parameter $n T \tau$. The neutron yield in this type of solutions diverges, and consequently the condition $\chi_{\text {no } \alpha}>1$ has been taken as a dynamic ignition criterion. Dynamic refers to the fact that the whole evolution of the hot spot expanding against the shell is considered when establishing the criterion, contrary to static ignition criteria which are based on the hot spot state at the stagnation moment. The latter do not take into account the tamping effect of the shell nor the evolution of the thermodynamic quantities in the hot spot, which are accounted for in the dynamic criterion. Since it is not necessary to request for infinite neutron yield when designing implosions, the dynamic criterion shall be taken as useful metrics to measure the quality of an implosion, that is, the closer an implosion gets to $\chi_{\text {no } \alpha}=1$ the better, rather than a strict condition that must be satisfied.

In the article presented in this chapter, the same study has been extended to cylindrical targets with an external magnetic field in a MagLIF context. Conceptually, there are several differences between ICF and MagLIF hot spot dynamics after stagnation. In ICF targets, the shell enclosing the fuel, typically deuterium-tritium DT, is made of cryogenic DT. The alpha particles, born with a $3.5 \mathrm{MeV}$ energy, deposit 
some of their energy in the hot spot via collisions with electrons mainly, and are stopped by the dense shell. They deposit the rest of their energy at the inner surface of the shell, causing its temperature to increase and promoting mass ablation into the hot spot. The ablated mass is still fuel where more fusion reactions can occur. Therefore, as pointed out in Ref. [23], the remaining energy of the alpha particles that escape the hot spot and crash against the shell is recycled back through this ablation mechanism; and one can licitly assume that the alpha particles deposit all their energy in the hot spot. This is not he case in standard MagLIF implosions, where the liner is made of a low atomic number metal in pulsed-power-driven implosions [84], or plastic in laser-driven implosions [8]. As discussed in Chapter 5, the hot spot is formed of hot fuel enclosed by ablated liner material. The alpha particles escaping the fuel would deposit their energy in the ablated material and at the inner side of the liner, where no more nuclear reactions can be triggered. Hence, it is the dynamics of the fuel region rather than the whole hot spot what should be studied to derive an ignition criterion, and the fact that the alpha particles leave the fuel region shall be accordingly taken into account. It is at this point where the magnetic field plays an important role, since it can confine the alpha particles within the fuel region, promoting alpha heating.

The analysis of the motion of the alpha particles, together with the derivation of a static ignition criterion for magnetized implosions, has been performed in Ref. [9]. The alphas are decelerated by Coulomb collisions with the electrons by means of a dynamic friction, which is modeled as being directly proportional to the velocity of the alphas [29]. Additionally, the alpha motion is affected by the presence of a magnetic field. Based on the motion of individual alpha particles, the authors computed the fraction of energy deposited in the fuel. However, an important effect that the authors did not take into account is the reentry of the alpha particles into the fuel as a consequence of the spiral motion induced by the magnetic field. These results have been used to derive a static ignition criterion based on the requirement that, at stagnation, alpha heating must overcome conduction and Bremsstrahlung losses. This criterion imposes temperatures in the range of $T=7-10 \mathrm{keV}$ and magnetic field intensities satisfying $B R \geq(4.5-6.5) \times 10^{5} \mathrm{G} \cdot \mathrm{cm}$, where $R$ is the fuel radius at stagnation.

The novelties presented in this article are the review of the alpha particles motion in a MagLIF context from a kinetic theory point of view, which allows to include the re-entry of the alphas in the fuel; and the derivation of a dynamic ignition criterion for magnetized implosions in cylindrical geometry, which highlights the benefits of magnetizing the fuel.

\subsection{Statement of the problem}

The expansion after stagnation of an infinitely long cylindrical hot spot enclosed by a liner, sketched in Fig. 1 in the article, is considered. The motion is axisymmetric, 
being $r$ the radial distance and $t$ time, with $t=0$ the stagnation moment. During the implosion, liner material is expected to ablate and compress the fuel. Consequently, after stagnation, the hot spot would be composed of the fuel region, $r \leq R(t)$, and the ablated liner material, $R(t)<r<R_{h}(t)$.

The qualitative description of the motion goes as follows. The hot spot expands against the liner with a characteristic confinement time $\tau_{c}$ based on the hot spot pressure at stagnation $P_{0}$ and the liner mass per unit length $M_{l}, \tau_{c}=\sqrt{M_{l} / 2 \pi P_{0}}$. The Mach number is assumed to be small and the magnetic pressure is negligible, hence thermal pressure is uniform, $P=P(t)$. The hot spot is highly magnetized, mass ablation is strongly reduced and the fuel region becomes magnetically insulated. Plasma motion induced by heat conduction is suppressed and, consequently, it is crudely assumed that the ratio $R / R_{h}$ is kept constant during the expansion. Simultaneously with the expansion, nuclear reactions occur and alpha particles are born. Only the fraction of the energy of the alpha particles deposited in the fuel, $f_{\alpha}$, contributes to increase its energy, a process referred to as alpha heating. The alpha particles dynamics is fast compared to $\tau_{c}$, hence they move and deposit their energy as if the hot spot state was frozen at the stage of the expansion under consideration. However, the hot spot energy increase due to alpha heating occurs at the characteristic time $\tau_{\alpha h}$ as defined in the paper, which may be comparable or higher than the confinement time. It is the quotient of these two characteristic times, the parameter $\delta=\tau_{c} / \tau_{\alpha h}$, what governs the problem. It is equivalent to the parameter $\chi_{\text {no } \alpha}$ introduced in Ref. [23]. The magnetic field comes into play enhancing the fraction of energy deposited $f_{\alpha}$. Depending on $\delta$ and the magnetic field intensity at stagnation, two trajectories can be found. The ones where expansion losses dominate over the alpha heating and the hot spot undergoes an adiabatic expansion, referred to as "sub-ignited"; and the ones where alpha heating dominates and the hot spot pressure becomes singular in a finite time, denoted as "ignited". It is the passage from sub-ignited to ignited regimes what has been taken as dynamic ignition criterion, in accordance to the similar criterion defined for ICF implosions in Ref. [23].

\subsubsection{Methodology}

The evolution of pressure and the fuel radius is given by the energy equation established in the fuel region and the liner momentum equation, Eqs. (2) and (3) in the article, respectively. Heat conduction losses and Bremsstrahlung radiation have been neglected, and the compressibility effects in the liner have been disregarded. The magnetic flux is assumed to be conserved during the implosion. The equations are normalized with the pressure and fuel radius at stagnation, and the characteristic confinement time $\tau_{c}$. The dimensionless governing equations for the dimensionless pressure $\hat{P}$ and fuel radius $\hat{R}$ correspond to Eqs. (4) and (5). They are completed with the initial conditions at stagnation, shown in Eq. (7). 
The evolution of the alpha particles is described kinetically by means of its distribution function $F(\mathbf{x}, \mathbf{v}, t)$. It is assumed that the alphas only collide with electrons, therefore the motion of every alpha particle is independent of the others. Particularly, Eq. (8) describes the evolution of the distribution function of a set of alphas born at the same time $\tau=\tau^{*}$. The alpha particles are assumed to be born uniformly in the fuel region, and their velocity distribution is isotropic. Their motion has a clear geometric interpretation: every subset of alphas born with the same velocity direction moves as a rigid solid describing a damped helical trajectory, the features of which are given by the hot spot conditions at birth time $\tau^{*}$. The latter can be condensed into two parameters: $\bar{R}=R / l_{\alpha}$ and $b=R / r_{l}$, where $l_{\alpha}$ is the alpha particles mean free path and $r_{l}$ is the Larmor radius of the born alphas. The first parameter measures the collisionality of the hot spot and the second the magnetization of the alphas. The average energy that an alpha particle transfers to the fuel, $f_{\alpha}$, can be obtained from the distribution function as shown in Eq. (15), and its computation can be reduced to quadrature, Eq. (16). The average energy depends then on the two parameters $\bar{R}$ and $b$, which evolve in time since the hot spot conditions change during the expansion. Particularly, they are related to their initial values $\bar{R}_{0}$ and $b_{0}$ and to $\hat{P}$ and $\hat{R}$, as given by Eq. (25). Consequently, at every stage of the expansion, $f_{\alpha}(\bar{R}, b)$ has to be updated. The governing parameters of the problem stand therefore for the quotient between the confinement and alpha heating times $\delta, \bar{R}_{0}$ and $b_{0}$.

An asymptotic analysis of the integral in Eq. (16) has been performed to elucidate the behavior of $f_{\alpha}$ for several limits of $\bar{R}, b$ in Subsec. III $\mathrm{B}$ in the article. Both small and large $\bar{R}$ limits have been studied in unmagnetized targets $b=0$. However, the more interesting limit for MagLIF applications corresponds to a low collisional hot spot $\bar{R} \ll 1$, and both small and large $b$ values have been derived.

\subsection{Results}

The fraction of alpha energy deposited in the fuel $f_{\alpha}$ has been computed in Fig. 3(a). Increasing the fuel magnetization leads to a considerable improvement of the fraction. A useful simplified expression for $f_{\alpha}$ based on the asymptotic laws derived for low collision limit $\bar{R} \ll 1$ is shown in Eq. (22). It is compared to the numerical computation in Fig. 3(b), showing good agreement.

First, the case of unmagnetized targets has been analyzed, $b_{0}=0$. The ideal case of no alpha heating losses is studied for comparison purposes by artificially setting $f_{\alpha}=1$. The solution only depends on $\delta$, and a critical $\delta=\delta_{c r}^{i d}=1.66$ that separates ignited from sub-ignited solutions has been identified. However, when the alpha heating losses are considered, ignited solutions cannot take place for any $\delta$ value. This is due to the increase of the alpha particles mean free path when pressure increases, which leads to less energy deposited in the fuel. Therefore, the solution 
in the unmagnetized case always tends to an adiabatic expansion regardless of the values of $\delta$ and $\bar{R}_{0}$.

Fortunately, the addition of a magnetic field allows for ignited solutions to take place due to the confinement effect it exerts on the alpha particles, as shown in Fig. 5. Consequently, a dynamic ignition criterion can be defined in terms of a critical $\delta_{c r}$, which separates ignited form sub-ignited regimes. The value of $\delta_{c r}$ depends on $b_{0}$ and $\bar{R}_{0}$, as shown in Fig. 6. In the limit of low collisional hot spots, characteristic of MagLIF implosions, two asymptotic expressions of the ignition criterion have been derived. For moderate magnetization values at stagnation, the ignition criterion reads $\delta>\delta_{c r}=8.45 / b_{0}^{2}$, while for large magnetization $b_{0} \gg 1$, the magnetic field traps all the alpha particles inside the fuel and the ideal ignition criterion with no alpha heating losses is recovered $\delta>\delta_{c r}^{i d}=1.66$. Magnetizing the fuel relaxes the critical value of $\delta$ required for ignition. Although this requirement can serve as valuable metrics for the quality of MagLIF implosions, it is not necessary to satisfy to achieve significant yield. In Fig. 7, isocontours of neutron yield for sub-ignited solutions are plotted as a function of $\delta$ and $b_{0}$. It can be seen that magnetizing the fuel allows to relax significantly the requirements on the implosion $\delta$ for a given yield value. 



\title{
Alpha Heating Enhancement in MagLIF Targets: An Analytic Model
}

\author{
J. Paradela, ${ }^{1}$ F. García-Rubio, ${ }^{1,}$ a) and J. Sanz ${ }^{1}$ \\ E.T.S.I. Aeronáutica y del Espacio, Universidad Politécnica de Madrid, Madrid 28040, \\ Spain.
}

(Dated: 13 September 2018)

\begin{abstract}
In this article, a dynamic ignition criterion for magnetized cylindrical implosions is derived. The analysis of the hot spot dynamics after stagnation performed in [Christopherson et al., Phys. Plasmas 25, 012703 (2018)] for spherical implosions is extended to cylindrical geometry with the addition of an external magnetic field. The hot spot dynamics have been solved self-consistently with a kinetic model for the alpha particles motion. The magnetic field confines the alpha particles and enhances their energy deposition in the fuel. The ignition criterion is based on the identification of ignited solutions where pressure exhibits a singularity in finite time, and neutron yield diverges. It is formulated in terms of a normalized Lawson parameter and the magnetization of the alpha particles at stagnation. The beneficial effect of magnetizing the fuel to increase the neutron yield is computed.
\end{abstract}

\section{INTRODUCTION}

In the recently proposed Magnetized Liner Inertial Fusion scheme (MagLIF), a magnetized cylindrical capsule enclosed by a conductive liner is compressed by a pulsedpower machine ${ }^{1,2}$. The role of the magnetic field is to reduce the heat conduction $\operatorname{losses}^{3}$, and to enhance the alpha heating by trapping alpha particles in the hot fuel.

The first effect was investigated in a simple MagLIF fusion-like plasma by Velikovich et al. ${ }^{4}$. Later on, GarcíaRubio and Sanz ${ }^{5-7}$ took into account the effects of mass ablation and magnetic pressure in the same configuration. Alpha heating enhancement was investigated by Basko et al. in Ref. ${ }^{8}$, where an analysis of the alpha particles energy deposition process in a magnetized cylindrical target was performed. In particular, an expression quantifying the average energy that an alpha particle transfers to the hot spot before escaping was obtained as a function of two parameters: $\bar{R}=R / l_{\alpha}$ and $b=R / r_{l}$ ( $R$ is the radius of the hot spot, $l_{\alpha}$ is the alpha particles mean free path and $r_{l}$ is the Larmor radius for an alpha particle with $3.5 \mathrm{MeV}$ ). The authors derived a static ignition criterion imposing that at stagnation, alpha heating must exceed the energy losses due to heat conduction and Bremsstrahlung radiation. It was found that significant reduction of the $\rho R$ implosion requirement was obtained, compared to typical Lawson criterion, for magnetic field $B$ intensities satisfying $B R>(4.5-6.5) \cdot 10^{5} \mathrm{G} \cdot \mathrm{cm}$.

More recently, Christopherson et al. ${ }^{9}$ derived a dynamic ignition criterion in an Inertial Confinement Fusion (ICF) context by studying the evolution of the hot spot dynamics after stagnation. A change of behavior in the dynamics of the system was found for a critical value of the parameter $\chi_{\text {no } \alpha}=\tau_{\alpha h} / \tau_{c}$, where $\tau_{\alpha h}$ is the characteristic alpha heating time and $\tau_{c}$ is the confinement time. When $\chi_{\text {no } \alpha}<1$, the hot spot undergoes an adiabatic expansion, falling into a sub-ignited regime. However, when $\chi_{\text {no } \alpha}>1$, pressure exhibits a singularity

\footnotetext{
a)Electronic mail: fernando.garcia.rubio@upm.es
}

in finite time and the neutron yield diverges. This second regime was called ignited regime, and the threshold value $\chi_{\text {no } \alpha}=1$ was defined as the "ignition condition". A particular feature of this study is that alpha heating losses were argued to be recycled back to the hot spot due to the ablation of the cryogenic D-T shell, so all the energy from nuclear reactions was considered to remain in the hot spot.

The goal of the present work is to review alpha heating enhancement in the central hot fuel of a MagLIF target after stagnation, and derive a dynamic ignition criterion for MagLIF by identifying ignited solutions in the sense of Ref. 9. The main differences are the cylindrical geometry and magnetization of the target. Besides, as the liner is typically made of beryllium or aluminum, the material ablated into the hot spot is different from the fuel. Therefore, as stated in Ref. 8, we cannot consider that alpha heating losses are recycled back by the ablated liner material to the hot fuel region.

This paper is organized as follows: in Sec. II, we derive the governing equations for the expansion of a MagLIF fusion-like plasma after the stagnation phase. In Sec. III, we revisit the alpha particles energy deposition process analyzed in Ref. 8 from a kinetic-theory point of view. In Sec. IV, the governing equations are solved paying special attention to the evolution of parameters $\bar{R}$ and $b$. Based on it, the dynamic ignition criterion for magnetized cylindrical implosions is derived. Furthermore, the nuclear yield as a function of the initial value of parameter $b$ is computed. In Sec. V conclusions are drawn.

\section{GOVERNING EQUATIONS}

We consider the expansion after stagnation of an infinitely long cylindrical hot spot enclosed by a liner, as represented in Fig. 1. We assume that liner material has been ablated during the implosion, hence at stagnation the hot spot is composed of the fuel region, $r \leq R$, and the liner material region, $R<r<R_{h}$. The fuel is magnetically insulated and mass ablation is strongly re- 


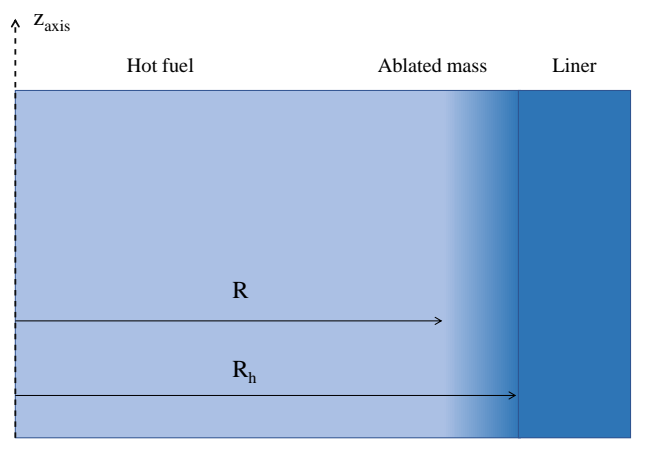

FIG. 1. Artistic scheme of a MagLIF target after stagnation. Axial symmetry is assumed. Color degradation represents density. From left to right: the hot fuel where nuclear reactions take place, the ablated material from the liner and the solid liner.

duced with magnetization ${ }^{7}$. Consequently, we make the crude assumption that $R / R_{h}$ is kept constant during the expansion.

We assume low Mach number hypothesis and negligible magnetic pressure. Thermal pressure in the hot spot is therefore uniform, $P=P(t)$. The evolution of the pressure $P(t)$ and volume $V(t)$ of the fuel is then given by the energy equation established in the fuel region and the momentum equation for the liner. In the energy balance, we neglect the fuel kinetic energy (subsonic approximation), heat conduction losses and Bremsstrahlung radiation. The reason for neglecting conduction losses is the high magnetization of the plasma, which substantially reduces heat conduction in the perpendicular direction to the magnetic field ${ }^{10}$. With these assumptions, the energy equation for the fuel reads:

$$
\frac{3}{2} \frac{\mathrm{d}(P V)}{\mathrm{d} t}=f_{\alpha} \frac{n^{2}}{4}\langle\sigma v\rangle \varepsilon_{\alpha} V-P \frac{\mathrm{d} V}{\mathrm{~d} t}
$$

where $n$ is the total (deuterium+tritium) ion particle density, $\langle\sigma v\rangle$ is the fusion reactivity, $\varepsilon_{\alpha}=3.5 \mathrm{MeV}$ is the birth energy for an alpha particle and $f_{\alpha}$ is the average energy that an alpha particle deposits inside the hot fuel volume $V$. It is important to notice that $f_{\alpha}$ depends on the instantaneous state of the fuel and the magnetic field intensity $B$, so it will evolve during expansion. Using the equation of state $P=2 n T$, and particularizing for cylindrical geometry $V=\pi R^{2}$, Eq. (1) transforms into:

$$
\frac{3}{2} \frac{\mathrm{d}\left(P R^{2}\right)}{\mathrm{d} t}=f_{\alpha} P^{2} \frac{\langle\sigma v\rangle}{16 T^{2}} \varepsilon_{\alpha} R^{2}-P \frac{\mathrm{d} R^{2}}{\mathrm{~d} t}
$$

In the typical range of temperatures considered $(T \sim$ $6-10 \mathrm{KeV}),\langle\sigma v\rangle \sim T^{2}$, and we can take $S_{\alpha}=$
$24 T^{2} /\langle\sigma v\rangle \varepsilon_{\alpha}$ as a constant. The momentum equation for the liner reads

$$
P 2 \pi R_{h}=M_{l} \frac{\mathrm{d}^{2} R_{h}}{\mathrm{~d} t^{2}}
$$

with $M_{l}$ being the liner mass per unit length in the axial direction. This equation can be expressed in terms of the fuel radius $R$ since, as commented before, we assume $R / R_{h}=a=$ const. during the expansion. Introducing the non-dimensional variables $\hat{P}=P / P_{0}, \hat{R}=R / R_{0}$, and $\tau=t / \tau_{c}$, with $P_{0}$ and $R_{0}$ being the pressure and radius of the fuel at stagnation, respectively, and $\tau_{c}$ being the characteristic confinement time $\tau_{c}=\sqrt{M_{l} / 2 \pi P_{0}}$, Eqs. (2),(3) transform into:

$$
\frac{\mathrm{d} \hat{P}}{\mathrm{~d} \tau}=\delta f_{\alpha} \hat{P}^{2}-\frac{10}{3} \frac{\hat{P}}{\hat{R}} \frac{\mathrm{d} \hat{R}}{\mathrm{~d} \tau},
$$

$$
\frac{\mathrm{d}^{2} \hat{R}}{\mathrm{~d} \tau^{2}}=\hat{P} \hat{R}
$$

Here, the parameter $\delta$ is the quotient between the confinement time and the characteristic alpha heating time $\tau_{\alpha h}=S_{\alpha} / P_{0}$,

$$
\delta=\frac{\tau_{c}}{\tau_{\alpha h}}=\sqrt{\frac{M_{l} P_{0}}{2 \pi}} \frac{1}{S_{\alpha}} .
$$

It is similar to the $\chi_{\text {no } \alpha}$ parameter defined in Ref. 9. As commented in this reference, $\delta$ is a normalized form of the typical Lawson parameter $n T \tau_{c}{ }^{11}$. This dynamical system is completed with the initial conditions at stagnation:

$$
\hat{P}(0)=1, \quad \hat{R}(0)=1, \quad \frac{d \hat{R}}{d \tau}(0)=0 .
$$

\section{ALPHA ENERGY DEPOSITION MODEL}

In order to close the system of equations, the average energy that an alpha particle deposits in the fuel, $f_{\alpha}$, needs to be derived.

In the typical range of temperatures considered, an alpha particle transfers its energy to the hot spot mainly via Coulomb collisions with the electrons. As stated in Ref. 8, we assume that these interactions decelerate the alphas with a dynamic friction force proportional to the alpha particle velocity, with a friction coefficient $\nu_{\alpha}=$ $v_{\alpha 0} / l_{\alpha}$ and $v_{\alpha 0}, l_{\alpha}$ being the alpha particle birth velocity and mean free path. The time scale for alpha energy deposition scales then as $1 / \nu_{\alpha}$. For simplicity, we also assume that the collision frequency $\nu_{\alpha}$ is the same inside and outside the fuel region. 


\section{A. Derivation of $f_{\alpha}$}

We use the kinetic theory to describe the evolution of the ensemble of alpha particles born at time $\tau=\tau^{*}$ in terms of a distribution function $F(\mathbf{x}, \mathbf{v}, t)$. Since the time scale for their energy deposition is very small compared to the macroscopic times $\tau_{c}, \tau_{\alpha h}$, we can assume that the hot spot conditions (magnetic field intensity, hot spot radius and collision frequency) are kept constant during the motion of an alpha particle. For this reason, we use the "alpha particle" time variable $t$ rather than the macroscopic time variable $\tau$ to describe their motion. Since the alpha particle collisions with themselves are less frequent than collisions with the surrounding plasma electrons, every set of alpha particles born at a given time evolves independently of the ones born sooner and later. Using the Einstein summation convention, the Boltzmann kinetic equation that describes the evolution of $F(\mathbf{x}, \mathbf{v}, t)$ reads then

$$
\frac{\partial F}{\partial t}+\frac{\partial F}{\partial x_{i}} v_{i}+\frac{\partial F}{\partial v_{i}}\left(\omega_{\alpha} v_{j} \varepsilon_{i j 3}-\nu_{\alpha} v_{i}\right)=3 \nu_{\alpha} F
$$

with $\omega_{\alpha}=2 e B / \mathrm{cm}_{\alpha}$ being the Larmor frequency for an alpha particle, $\varepsilon_{i j 3}$ standing for the Levi-Civita symbol and $i=1,2,3$ with 3 representing the axial direction. The term on the right hand of the equation is due to the non-conservative friction force. We introduce the nondimensional variables $\hat{x}_{i}=x_{i} / R, \hat{t}=v_{\alpha 0} t / R, \hat{v}_{i}=v_{i} / v_{\alpha 0}$ and the two dimensionless parameters $b=R \omega_{\alpha} / v_{\alpha 0}=$ $R / r_{l}$ and $\bar{R}=R / l_{\alpha}$. Notice that the alpha particles magnetization is directly related to these parameters since $\omega_{\alpha} \tau_{\alpha}=\omega_{\alpha} l_{\alpha} / v_{\alpha 0}=b / \bar{R}$. The Boltzmann equation written in these new variables gives

$$
\frac{\partial F}{\partial \hat{t}}+\hat{v}_{i} \frac{\partial F}{\partial \hat{x}_{i}}+\left(b \hat{v}_{j} \varepsilon_{i j 3}-\bar{R} \hat{v}_{i}\right) \frac{\partial F}{\partial \hat{v}_{i}}=3 \bar{R} F .
$$

Notice that the hot spot conditions at birth time $\tau^{*}$ are condensed into $b, \bar{R}$. The former equation needs to be complemented with the corresponding initial condition. We consider that the alpha particles are born inside the fuel with a birth rate that is proportional to $n^{2}\langle\sigma v\rangle \sim$ $n^{2} T^{2} \sim P^{2}$, which is uniform, and that their velocity distribution is isotropic. The initial distribution function corresponds then to a cylinder in the position phase space occupying the fuel region $\hat{x}_{1}^{2}+\hat{x}_{2}^{2} \leq 1$,

$$
F(\hat{\mathbf{x}}, \hat{\mathbf{v}}, 0)=\frac{\delta(\hat{v}-1)}{4 \pi} \frac{1}{\pi} H\left(1-\hat{x}_{1}^{2}-\hat{x}_{2}^{2}\right),
$$

where $\delta, H$ are the Dirac delta function and the Heaviside function, respectively. The solution $F(\hat{\mathbf{x}}, \hat{\mathbf{v}}, \hat{t})$ can be obtained by the method of characteristics, reading

$$
\begin{aligned}
F(\hat{\mathbf{x}}, \hat{\mathbf{v}}, \hat{t})=\frac{\delta\left(\hat{v}-e^{-\bar{R} \hat{t}}\right)}{4 \pi \hat{v}^{2}} & \frac{1}{\pi} H[1- \\
& \left.\left(\hat{x}_{1}-d_{1}\right)^{2}-\left(\hat{x}_{2}-d_{2}\right)^{2}\right],
\end{aligned}
$$

with

$$
\begin{aligned}
& d_{1}=\frac{e^{\bar{R} \hat{t}}}{\bar{R}^{2}+b^{2}} {\left[\left(\bar{R} \hat{v}_{1}+b \hat{v}_{2}\right) \cos (b \hat{t})\right.} \\
&\left.+\left(b \hat{v}_{1}-\bar{R} \hat{v}_{2}\right) \sin (b \hat{t})\right]-\frac{\bar{R} \hat{v}_{1}+b \hat{v}_{2}}{\bar{R}^{2}+b^{2}}
\end{aligned}
$$

and

$$
\begin{aligned}
& d_{2}=\frac{e^{\bar{R} \hat{t}}}{\bar{R}^{2}+b^{2}} {\left[\left(\bar{R} \hat{v}_{2}-b \hat{v}_{1}\right) \cos (b \hat{t})\right.} \\
&\left.+\left(\bar{R} \hat{v}_{1}+b \hat{v}_{2}\right) \sin (b \hat{t})\right]-\frac{\bar{R} \hat{v}_{2}-b \hat{v}_{1}}{\bar{R}^{2}+b^{2}} .
\end{aligned}
$$

The structure of the distribution function in the position phase space is such that for a given $\{\mathbf{v}, t\}$, it corresponds to a cylinder centered in $\left\{d_{1}, d_{2}\right\}$. Besides, since the coefficients in Eq. (9) only depend on $\mathbf{v}$, the equations for the characteristic lines establish a bijection between the velocity at a given time and its initial value. Consequently, the aforementioned cylinder is composed of alpha particles born with the same velocity direction that are transported as a rigid body, following a helical damped trajectory. The initial distribution can therefore be decomposed in subsets of particles born with the same velocity direction, each subset describing a different helical damped trajectory, as sketched in Fig. 2(a).

An alpha particle moving in these conditions decreases its kinetic energy $E_{\alpha}=m_{\alpha} v^{2} / 2$ due to the friction with the fuel according to

$$
\frac{1}{\frac{1}{2} m_{\alpha} v_{\alpha 0}^{2}} \frac{\mathrm{d} E_{\alpha}}{\mathrm{d} \hat{t}}=2 \bar{R} \hat{v}^{2}=2 \bar{R} e^{-2 \bar{R} \hat{t}} .
$$

The average energy that an alpha particle transfers to the fuel, $f_{\alpha}$, is thereby obtained as

$$
f_{\alpha}=\int_{0}^{\infty} 2 \bar{R} e^{-2 \bar{R} \hat{t}} \int_{\Omega} F(\hat{\boldsymbol{x}}, \hat{\boldsymbol{v}}, \hat{t}) \mathrm{d} \hat{\mathbf{x}} \mathrm{d} \hat{\mathbf{v}} \mathrm{d} \hat{t},
$$

where the integration domain in the phase space is $\Omega=$ $\left\{(\hat{\mathbf{x}}, \hat{\mathbf{v}}) ; \hat{x}_{1}^{2}+\hat{x}_{2}^{2} \leq 1, \hat{v}^{2}<\infty\right\}$.

The integral (15) can be thought of as computing the intersection between every cylinder describing the aforementioned motion with the fuel region, $\hat{x}_{1}^{2}+\hat{x}_{2}^{2} \leq 1$, and averaging over all the initial velocity directions. Due to the symmetry of the problem, this average only needs to be performed over the pitch angle $\theta$, see Fig. 2(b). The velocity pitch angle is preserved throughout the motion of the particles. Letting $\Delta(\theta, \hat{t})$ represent this intersection, the integral for $f_{\alpha}$ can be expressed as

$$
f_{\alpha}=\frac{1}{\pi} \int_{0}^{\infty} 2 \bar{R} e^{-2 \bar{R} \hat{t}} \int_{0}^{\pi / 2} \Delta(\theta, \hat{t}) \sin \theta \mathrm{d} \theta \mathrm{d} \hat{t} .
$$


(a)

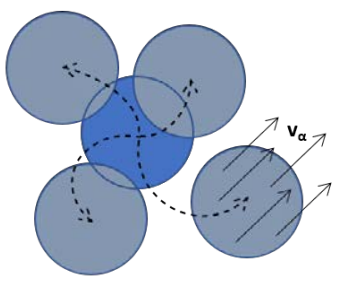

(b)

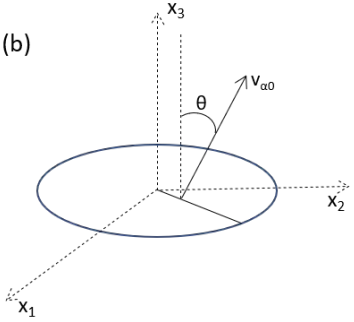

FIG. 2. (a) Sketch of the motion of the sets of alpha particles with same initial velocity. View from the z-axis. (b) Pitch angle $\theta$.

The intersection $\Delta(\theta, \hat{t})$ can be obtained from distance between the cylinder centers $d$,

$$
\begin{aligned}
& d(\theta, \hat{t})=\sqrt{d_{1}^{2}+d_{2}^{2}}=\frac{\sin \theta}{\left(\bar{R}^{2}+b^{2}\right)^{1 / 2}} \times \\
&\left(1-2 e^{-\bar{R} \hat{t}} \cos (b \hat{t})+e^{-2 \bar{R} \hat{t}}\right)^{1 / 2},
\end{aligned}
$$

giving

$$
\begin{aligned}
\Delta(\theta, \hat{t})=\left[\arccos \left(\frac{d}{2}\right)-\right. & \\
& \left.\frac{d}{2} \sqrt{1-\left(\frac{d}{2}\right)^{2}}\right] H\left(1-\frac{d}{2}\right) .
\end{aligned}
$$

The difference between this analysis and the one performed in Ref. 8 is that this formulation takes into account the reentry of alpha particles due to the effect of the magnetic field. This is represented in the oscillatory part of the distance function $d(\theta, \hat{t})$. Nevertheless, this is still a vague approximation since we have considered that the fuel and the surrounding plasma have the same magnetic field and collision frequency. A different characterization of the liner ablated material would make the resolution of Eq. (9) rather cumbersome and is beyond the scope of this work.

In a more realistic picture, we can consider that not all the alpha particles that escape the fuel are subject to re-enter, since the ones traveling far enough would be stopped by the dense liner. We can crudely take this into account by not computing the reentry of those cylinders that completely escape the fuel region, which assumes that the dense liner is placed at three fuel region radius: $R_{h} / R=3$. Mathematically, this would be done by changing the argument of the Heaviside function in Eq. (18) to $H\left(1-t_{\text {esc }}\right)$, where the escape time $t_{\text {esc }}$ is defined as the time when a set of alpha particles with pitch angle $\theta$ completely exits the fuel region, $d\left(\theta, t_{\mathrm{esc}}\right)=2$. Hereinafter, the $f_{\alpha}$ computed this way would be denoted "absorbed energy with partial reentries", contrary to "absorbed energy with full reentries" when we take into account all of them.

\section{B. Asymptotic analysis}

The absorbed energy $f_{\alpha}$ only depends on $\bar{R}$ and $b$. We proceed to derive its asymptotic behavior in different limits. We start with the simplest case that corresponds to the unmagnetized target, $b=0$. In this limit, there is no reentry and, consequently, we obtain the same results as in Ref. 8,

$$
\left.f_{\alpha}\right|_{b=0}= \begin{cases}\frac{8}{3} \bar{R}+\text { h.o.t. } & \text { if } \bar{R} \ll 1, \\ 1-\frac{1}{6 \bar{R}}+\text { h.o.t. } & \text { if } \bar{R} \gg 1 .\end{cases}
$$

For the magnetized case, $b \neq 0$, we restrict our analysis to the limit $\bar{R} \ll 1$, since this is the most representative situation in a realistic MagLIF configuration, as discussed in Subsec. (IV). In this low collision frequency limit, the magnetic field plays an important role confining the alphas. For $b \geq 1$, no cylinder escapes completely the fuel, and the "partial reentry" and "full reentry" cases coincide. Particularly, we can derive the following asymptotic limit for large magnetization $b \gg 1$

$$
f_{\alpha}=1-\frac{0.56}{b}+\text { h.o.t. } \quad \text { if } \bar{R} \ll 1 \ll b \text {. }
$$

This result agrees with the estimation of Ref. 8, $f_{\alpha} \sim 1-O(1 / b)$, but the value of the numerical coefficient, result of the integral $I=$ $\int_{0}^{\infty} e^{-2 \tau} \sqrt{1-2 e^{-\tau} \cos (b \tau / \bar{R})+e^{-2 \tau}} \mathrm{d} \tau$ in the limit $b / \bar{R} \rightarrow \infty$, accounts for the re-entry of alpha particles.

When the magnetization is low, $b \ll 1$, it is useful to divide the alphas into two groups ${ }^{8}$, those whose velocity is nearly axial, $0 \leq \theta \leq \theta_{c}$, and the rest. The former define a "capture cone" and deposit a significant fraction of their energy, $\sim O(1)$, in the fuel. The size of the capture cone is $\theta_{c} \sim \bar{R}$ if $b \ll \bar{R} \ll 1$, and $\theta_{c} \sim b$ if $\bar{R} \ll$ $b \ll 1$. As a result, the energy deposition contribution of these alphas is of order $O\left(\bar{R}^{2}\right)$ and $O\left(b^{2}\right)$, respectively. The rest of the alphas completely escape the fuel. In the "partial reentry" assumption, their contribution to energy is of order $O(\bar{R})$. We find then that for $\bar{R} \ll b^{2} \ll$ 1 , most of the energy deposited comes from the capture cone alphas, and we have $f_{\alpha}=0.27 b^{2}+$ h.o.t.; while for $b^{2} \ll \bar{R} \ll 1$, most of the energy deposited comes from the rest of the alphas, and we have $f_{\alpha}=8 \bar{R} / 3+$ h.o.t.. These two limits can be merged into one formula

$$
f_{\alpha} \simeq \frac{8}{3} \bar{R}+0.27 b^{2} \text { if } \bar{R}, b \ll 1 .
$$

Again, this results are in accordance with the estimations of Ref. $8, f_{\alpha} \sim 8 \bar{R} / 3+O\left(b^{2}\right) ;$ and the numerical coefficient, result of the integral $J=$ $\frac{16}{\pi} \int_{0}^{\infty} \int_{0}^{1 / 2} e^{-2 \tau}\left(\arccos \alpha-\alpha \sqrt{1-\alpha^{2}}\right) \varphi \mathrm{d} \tau \mathrm{d} \varphi$, with $\alpha=$ $\varphi\left[1-2 e^{-\tau} \cos (b \tau / \bar{R})+e^{-2 \tau}\right]$, in the limit $b / \bar{R} \rightarrow \infty$, accounts for reentry of alpha particles. 


\section{Numerical results}

The average absorbed energy $f_{\alpha}$ given by the "partial reentry" formulation is depicted in Fig. 3(a) against magnetization $b$ for different $\bar{R}$ values. It can be noticed that there is an abrupt slope change at $b=1$. For larger magnetization values, the magnetic field confines all the alpha particles inside the hot spot, and can reenter into the fuel after escaping. However, for lower values, the confinement is not strong enough and some alpha particles reach the dense liner, depositing there all their energy. The asymptotic behavior for small and large $b$ keeping $\bar{R} \ll 1$ is plotted in dashed lines, showing excellent agreement even for values of $b$ of order unity.

In Fig. 3(b), the absorbed energy $f_{\alpha}$ is computed in the "partial reentry" and "full reentry" formulations, and is compared to the formulation with no reentry performed in Ref. 8. It can be seen that the energy deposition contribution due to the alpha particles reentry is important. However, taking into account all the latter significantly overestimates the absorbed energy, since it ignores presence of the dense liner. For this reason, hereinafter we use the "partial reentry" formulation results. Based on the asymptotic analysis performed before, we can derive an analytical approximation for $f_{\alpha}$ in the $\bar{R} \ll 1$ limit:

$$
f_{\alpha}=\left\{\begin{array}{ll}
\frac{8}{3} \bar{R}+0.27 b^{2}+0.17 b^{6}, & \text { if } b \leq 1, \\
1-\frac{0.56}{b}, & \text { if } b>1
\end{array} 22\right.
$$

This approximation is more than $10 \%$ accurate. It is plotted in Fig. 3(b) together with the analytic formula given in Eq. (15) in Ref. 8.

\section{FUEL DYNAMICS AFTER STAGNATION}

We consider now the system of equations that describes the evolution of the fuel after stagnation, Eqs. (4) and (5), which has been closed with the derivation of $f_{\alpha}(\bar{R}, b)$. The parameters $\bar{R}$ and $b$ evolve during the fuel expansion. Considering that

$$
\begin{gathered}
l_{\alpha}=\frac{3}{4 \sqrt{2 \pi}} \frac{m_{\alpha} v_{\alpha 0} T^{3 / 2}}{Z_{\alpha}^{2} e^{4} m_{e}^{1 / 2} L_{\alpha} n} \sim \frac{T^{3 / 2}}{n} \sim R^{5} P^{3 / 2}, \\
r_{l}=\frac{v_{\alpha 0} m_{\alpha} c}{2 e B} \sim \frac{1}{B} \sim R^{2},
\end{gathered}
$$

the parameters $\bar{R}$ and $b$ evolve as

$$
\bar{R}=\frac{\bar{R}_{0}}{\hat{R}^{4} \hat{P}^{3 / 2}}, \quad b=\frac{b_{0}}{\hat{R}},
$$

with $\bar{R}_{0}$ and $b_{0}$ being their initial value at stagnation. Notice that it has been assumed that the fuel mass and magnetic flux, $n R^{2}$ and $B R^{2}$, are conserved during the
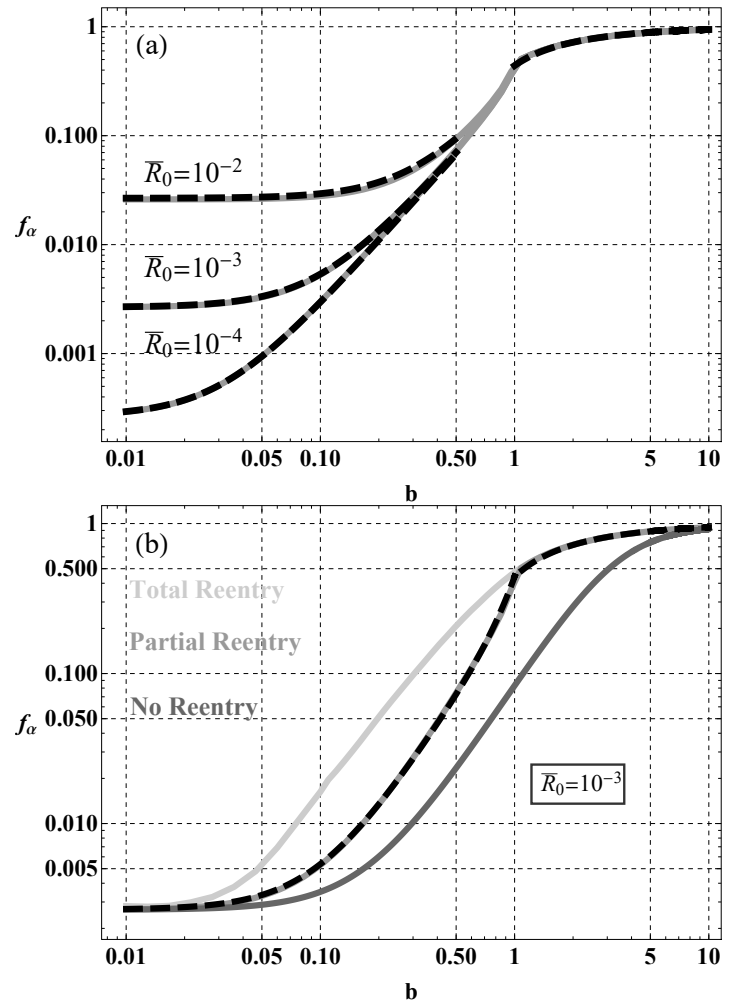

FIG. 3. (a) Average alpha particles absorbed energy $f_{\alpha}$ as a function of magnetization $b$ for different values of $\bar{R}$, computed with the "partial reentry" formulation. The asymptotic limits Eqs. (20) and (21) are plotted in dashed lines. (b) Average alpha particles absorbed energy $f_{\alpha}$ computed with the "partial reentry", "total reentry" and "no reentry" formulations for $f_{\alpha}$. The analytic expression (22) is plotted in dashed lines.

expansion, which is a reasonable assumption since the fuel is highly magnetized.

The system of governing equations depends then on three free parameters: $\delta, \bar{R}_{0}$ and $b_{0}$. Practical expressions (in C.G.S. units) for these parameters are

$$
\bar{R}_{0}=9.34 L_{\alpha} \frac{\rho R}{T_{\mathrm{keV}}^{3 / 2}} \quad b_{0}=3.69 \cdot 10^{-6} B R .
$$

Taking $L_{\alpha}=7$ for the Coulomb Logarithm and typical values $\rho=3 \mathrm{~g} / \mathrm{cm}^{3}, R=8 \cdot 10^{-3} \mathrm{~cm}, T=10 \mathrm{keV}, B=$ $10^{8} \mathrm{G}$, we obtain

$$
\bar{R}_{0}=0.049, \quad b_{0}=2.95
$$

We anticipate the behavior of the solution $\hat{P}(\tau), \hat{R}(\tau)$. At stagnation, alpha heating overcomes expansion losses (PdV work) in the energy balance (4) and pressure increases, pushing the liner outwards. Then, two different regimes can be identified depending on the value of $\delta$. If the expansion losses dominate, the solution tends to an adiabatic expansion $\hat{P} \sim \hat{R}^{-10 / 3}$. This solution takes 
place for $\delta$ lower than a critical value $\delta_{c r}$. If alpha heating dominates, which happens for $\delta>\delta_{c r}$, pressure shows a singularity in a finite time $\tau_{s}$ of the kind $\hat{P} \sim 1 /\left(\tau_{s}-\tau\right)$. As introduced in Ref. 9, we refer to the former solutions as "sub-ignited" and to the latter as "ignited".

\section{A. Unmagnetized fuel}

We first analyze the simple unmagnetized case $b_{0}=0$. The numerical solution of the governing equations are depicted in Fig. 4 for $b=0, \bar{R}_{0}=10^{-2}$ and different values of $\delta$. In order to highlight the importance of the alpha particles energy losses, we compare the model with the ideal case without losses, that is, $f_{\alpha}=1$.

In the ideal case, we can identify a critical value $\delta_{c r}^{i d} \approx$ 1.66 that separates the ignited and sub-ignited solutions. For $\delta=\delta_{c r}^{i d}$, the solution tends to the separatrix $\hat{P}=$ $\left(10 / 3 \delta_{c r}^{i d}\right)^{2} \approx 4.03$, plotted with dashed lines in Fig. (4). For $\delta>\delta_{c r}^{i d}$, the solutions are ignited and they result in an infinite neutron yield, hence $\delta=\delta_{c r}^{i d}$ can be seen as a dynamic ignition criterion, equivalently to the one derived in Ref. 9.

When the alpha particles energy losses are considered, ignited solutions cannot take place for any $\delta$ value. This can be proved by reduction to the absurd. If an ignited solution takes place, pressure would increase in time, alpha particles mean free path would become larger and the parameter $\bar{R}$ would decrease as given by Eq. (25). The absorbed energy would behave then as $f_{\alpha} \approx 8 \bar{R} / 3$. If the alpha energy deposition has to overcome the expansion losses, the following inequality would have to be satisfied,

$$
\delta \frac{8}{3} \frac{\bar{R}_{0}}{\hat{R}^{4} \hat{P}^{3 / 2}} \hat{P}^{2}>\frac{10}{3} \hat{P} \hat{R} \frac{\mathrm{d} \hat{R}}{\mathrm{~d} \tau}
$$

Using Eq. (5) for $\mathrm{d} \bar{R} / \mathrm{d} \tau$, this condition can be rewritten as

$$
\left(\frac{4}{5} \delta \bar{R}_{0}\right) \frac{1}{\hat{R}^{5} \hat{P}^{1 / 2}}>\int \hat{P} \hat{R} \mathrm{~d} \tau
$$

which cannot hold for a sufficiently long time $\tau$ since pressure is an increasing function of time. Therefore, the solution in the unmagnetized case taking into account alpha losses always tends to an adiabatic expansion regardless of the value of $\delta$ and $\bar{R}_{0}$.

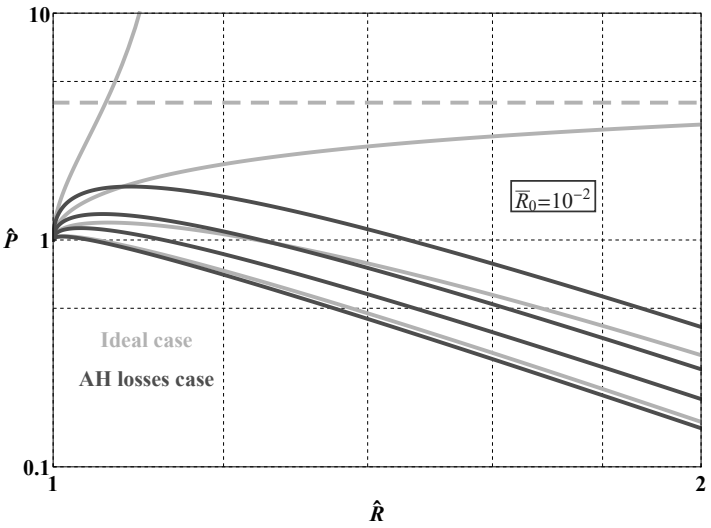

FIG. 4. Curves $\hat{P}(\hat{R})$ for an expanding unmagnetized fuel. Light colored curves correspond to the ideal case with different values of $\delta=0.5,1, \delta_{c r}^{i d}, 3$, where $\delta_{c r}^{i d}=1.66$. Dark curves correspond to the real case with alpha heating losses for different values of $\delta^{*}=8 \bar{R}_{0} \delta / 3=0.5,1, \delta_{c r}^{i d}, 3$.

\section{B. Magnetized fuel}

Magnetization of the fuel is intended to enhance alpha particles energy deposition. In Fig. $5, \hat{P}(\hat{R})$ curves are shown for $\bar{R}_{0}=10^{-2}, b_{0}=0.8$ and different $\delta$ values. It can be seen that the magnetic field confinement effect on the alpha particles allows for ignited solutions to take place.

If we assume a moderate magnetization at stagnation, $\bar{R}_{0} \ll b_{0}<1$, we can approximate $f_{\alpha} \approx 0.27 b^{2}=$ $0.27 b_{0}^{2} / \hat{R}^{2}$. The governing parameters can be condensed into one, $\psi \equiv 0.27 \delta b_{0}^{2}$, and we can identify a critical solution $\hat{P}=484 \hat{R}^{4} / 27 \psi_{c r}^{2}$, where $\psi_{c r} \equiv 0.27 \delta_{c r} b_{0}^{2} \approx 2.28$. Trajectories with $\delta>\delta_{c r}$ are ignited and present a pressure singularity in finite time, whereas trajectories with $\delta<\delta_{c r}$ are sub-ignited and fall into an adiabatic expansion. Notice that in the critical solution, $\bar{R} \ll b$ for all time, and the previous approximation of $f_{\alpha}$ is justified. We can therefore derive a simple scaling for the effect of magnetization on the ignition requirements,

$$
\delta_{c r} \approx \frac{8.45}{b_{0}^{2}}, \quad \text { if } \bar{R}_{0} \ll b_{0}<1 .
$$

For higher $b_{0}$ values, the alpha particles are strongly confined into the fuel region, and we recover the ideal solution with $f_{\alpha}=1$, hence $\delta_{c r} \rightarrow \delta_{c r}^{i d}$. In Fig. 6 , the value of $\delta_{c r}$ is plotted against the magnetization at stagnation. It can be seen that both limits agree well with the numerical results, and the value of $\delta_{c r}$ is reduced with $\bar{R}_{0}$.

An important parameter in ICF implosions is the amount of thermonuclear reactions taking place, usually referred to as neutron yield $Y$. It can be computed as

$$
Y=\int_{0}^{\infty} V P^{2} \frac{\langle\sigma v\rangle}{16 T^{2}} \varepsilon_{\alpha} \mathrm{d} t
$$




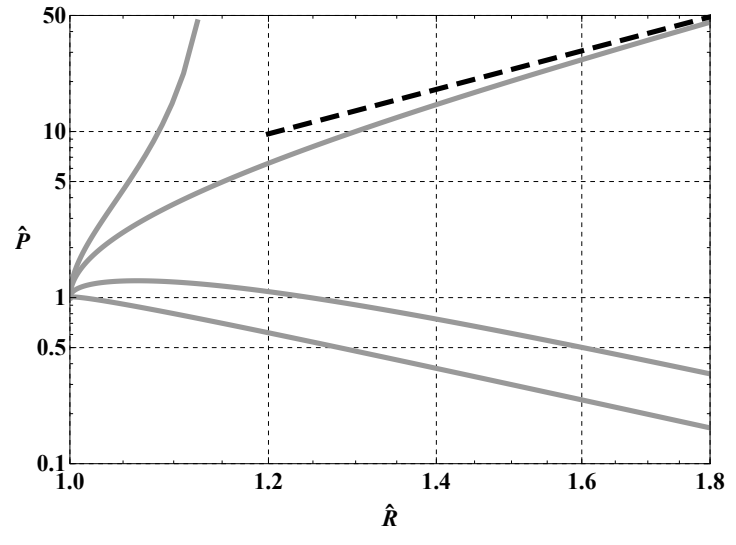

FIG. 5. Curves $\hat{P}(\hat{R})$ for an expanding magnetized hot fuel with $\bar{R}_{0}=10^{-2}, b_{0}=0.8$. Each curve corresponds to a different value of $\delta=1,5, \delta_{c r}, 15$, where $\delta_{c r}=11.33$ in this case. The critical solution $\hat{P}=\left(246 / \delta_{c r}^{2} b_{0}^{4}\right) \hat{R}^{4}$ is plotted in dashed black lines.

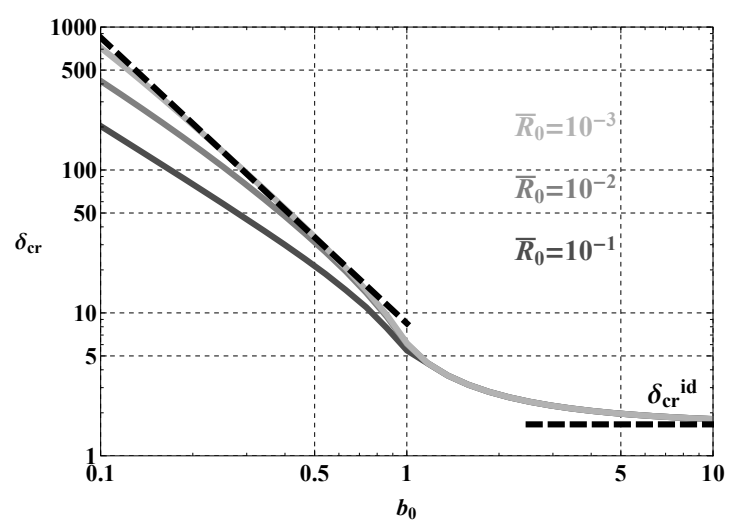

FIG. 6. Critical value $\delta_{c r}$ versus magnetization at stagnation $b_{0}$. The asymptotic law Eq. (28) is shown in dashed lines.

Notice that the neutron yield is infinite for ignited solutions, since they present a pressure singularity. Therefore, although the ignition condition derived can serve as valuable metrics for the quality of MagLIF implosions, it is not necessary to satisfy it to achieve a significant yield. In order to measure the alpha heating effect, the neutron yield is typically normalized with the yield resulting from an implosion where the alpha energy deposition contribution to the hydrodynamic is neglected, $Y_{\text {no }}{ }^{9}$. Isocontours of the ratio $Y / Y_{\text {noo }}$ are shown in Fig. (7) for $\bar{R}_{0}=10^{-3}$. It can be seen that increasing the initial magnetization $b_{0}$ significantly reduces the $\delta$ value needed to achieve a given yield ratio.

A simpler way to analyze the alpha heating effect is to compute the ratio between alpha heating in the hot fuel and its initial energy,

$$
G=\frac{\int_{0}^{\infty} f_{\alpha} P^{2} \frac{\langle\sigma v\rangle}{16 T^{2}} V \varepsilon_{\alpha} \mathrm{d} t}{\frac{3}{2} P_{0} V_{0}}
$$

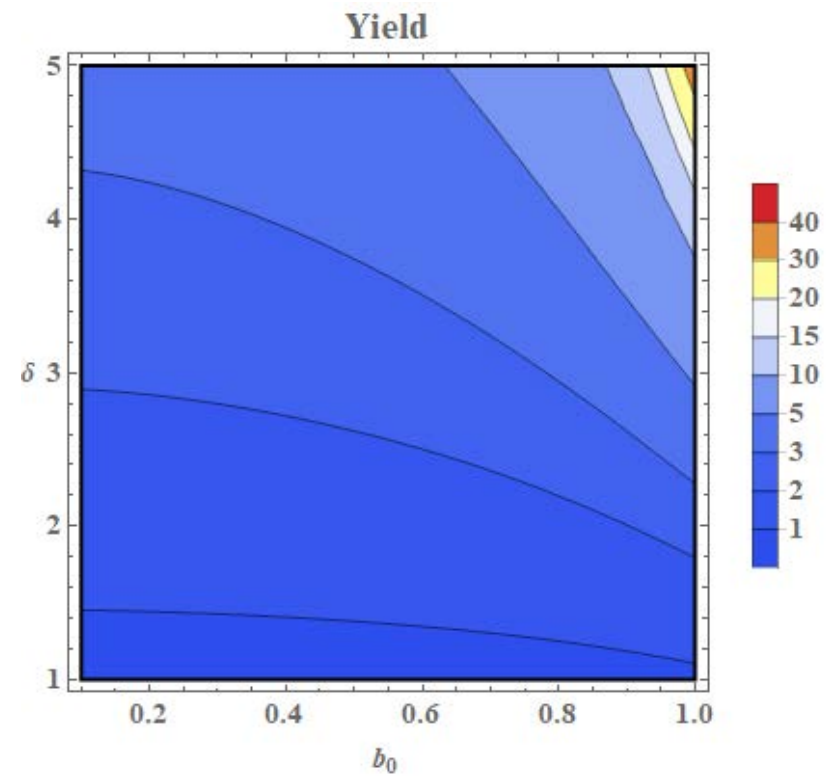

FIG. 7. Neutron yield ratio $Y / Y_{\text {no } \alpha}$ isocontours for $\bar{R}_{0}=$ $10^{-3}$.

For low magnetization $b_{0}<1$ and $\bar{R}_{0} \ll 1$ one can approximate $f_{\alpha} \approx 0.27 b^{2}$ and the aforementioned ratio between alpha heating and initial energy becomes a function depending only on $\psi$,

$$
G(\psi)=\psi \int_{0}^{\infty} \hat{P}^{2} \mathrm{~d} \hat{t} .
$$

For a fixed value of parameter $\delta, G$ increases with $b_{0}$, resulting in a direct measure of how alpha heating is enhanced by magnetization. In Fig. 8 we can observe that as $\psi$ approaches the critical value $\psi_{c r} \approx 2.28$ the ratio becomes infinite.

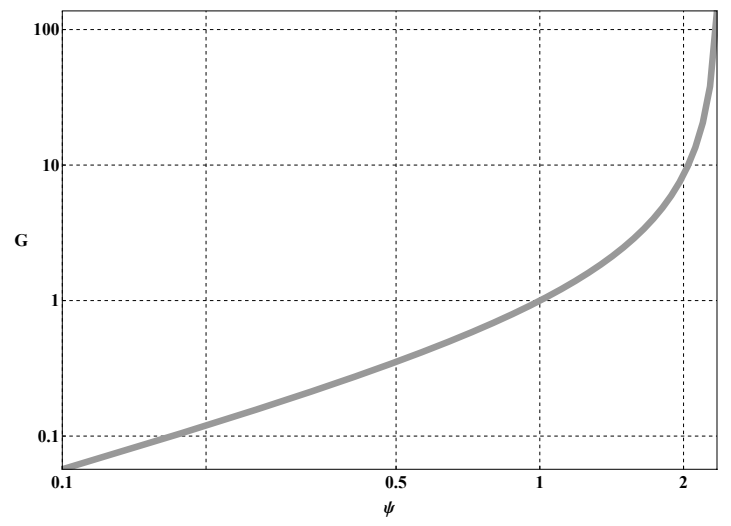

FIG. 8. Ratio between alpha heating in the hot fuel and its initial energy as a function of $\psi=0.27 \delta b_{0}^{2}$ for low magnetized targets. 


\section{CONCLUSIONS}

A new ignition criterion for magnetized cylindrical implosions has been derived based on the dynamics of the fuel after stagnation. In contrast to previous static ignition criterion derived for magnetized implosions, it takes into account the expansion losses (PdV work) and evolution of the mean free path of alpha particles, which has been shown to be very detrimental for achieving a significant yield.

The criterion is based on the existence of ignited solutions, where pressure exhibits a singularity in finite time. It is formulated in terms of the ratio between the alpha heating and confinement times, $\delta$, which stands for a normalized form of the Lawson parameter $n T \tau_{c}$, and the magnetization of the alpha particles at stagnation $b_{0}$. It has been proved that ignited solutions cannot exist for unmagnetized targets due to the decrease of alpha particles mean free path. The addition of a magnetic field confines the alpha particles and palliates the latter effect. With a sufficiently strong magnetic field, we can recover ignited solutions where alpha heating overcomes expansion losses and the pressure exhibits a singularity in finite time.

For moderate magnetized targets, the implosion requirements for achieving ignition are reduced with the magnetization level at stagnation $b_{0}$ as follows

$$
T \sim 6-10 \mathrm{keV}, \quad \delta b_{0}^{2}>8.45 \quad \text { when } \quad b_{0}<1
$$

whereas for high magnetization, the ignition criteria

$$
T \sim 6-10 \mathrm{keV}, \quad \delta>1.66 \text { when } \quad b_{0} \gg 1 .
$$

For low magnetized targets, the parameter $\psi=0.27 \delta b_{0}^{2}$ has also been proved to be a good metric of the energy ratio between absorbed nuclear energy in the hot spot and its initial energy.

\section{ACKNOWLEDGMENTS}

This research was supported by the Spanish Ministerio de Economía y Competitividad, Project No. ENE201454960R, and by the Spanish Ministerio de Educación, Cultura y Deporte under the national research program FPU, grant FPU 14/04879.

${ }^{1}$ S. A. Slutz, M. C. Herrmann, R. A. Vesey, A. B. Sefkow, D. B. Sinars, D. C. Rovang, K. J. Peterson, and M. E. Cuneo, Phys. Plasmas 17, 056303 (2010).

${ }^{2}$ M. R. Gomez, S. A. Slutz, A. B. Sefkow, D. B. Sinars, K. D. Hahn, S. B. Hansen, E. C. Harding, P. F. Knapp, P. F. Schmit, C. A. Jennings, et al., Phys. Rev. Lett. 113, 155003 (2014).

${ }^{3}$ R. Landshoff, Phys. Rev. 76, 904 (1949).

${ }^{4}$ A. L. Velikovich, J. L. Giuliani, and S. T. Zalesak, Phys. Plasmas 22, 042702 (2015).

${ }^{5}$ F. García-Rubio and J. Sanz, Physics of Plasmas 24, 072710 (2017).

${ }^{6}$ F. García-Rubio and J. Sanz, Physics of Plasmas 25, 042114 (2018).

${ }^{7}$ F. García-Rubio and J. Sanz, Physics of Plasmas 25, 082112 (2018).

${ }^{8}$ M. M. Basko, A. J. Kemp, and J. Meyer-ter Vehn, Nucl. Fusion 40, 59 (2000).

${ }^{9}$ A. R. Christopherson, R. Betti, A. Bose, J. Howard, K. M. Woo, E. M. Campbell, J. Sanz, and B. K. Spears, Physics of Plasmas 25, 012703 (2018).

${ }^{10}$ S. I. Braginskii, Reviews of Plasma Physics, edited by M. A. Leontovich (Consultants Bureau, New York, 1965).

${ }^{11}$ J. D. Lawson, Proceedings of the Physical Society. Section B 70, 6 (1957). 


\section{Conclusions}

In this thesis, several fundamental hydrodynamic processes arising in magnetized liner inertial fusion have been analyzed. Each of these processes has been studied by proposing a scenario which is simple enough to allow for an analytical treatment, but yet realistic enough to capture all the physics faithfully. The aim has been both to understand every key process from a fundamental point of view as well as to derive exact solutions that can serve as valuable test cases to validate and benchmark magnetohydrodynamics codes.

In Chapter 2, magnetized plasma expansion has been investigated under the ideal magnetohydrodynamics hypothesis. The expansion is a self-similar process in the variable $x / t$. The structure of the expansion is composed of nonlinear magnetosonic waves and plateaus matched through weak discontinuities. When the magnetic field inclination is perpendicular, the plasma expands through a fast magnetosonic wave, which is followed by a plateau where the plasma is piled up. The plasma reaches the vacuum at the end of the plateau with a finite density. When the magnetic field is oblique, both fast and slow magnetosonic waves coexist, the latter allowing the plasma to expand further, which reaches the vacuum with zero density. In this case, the Lorentz force triggers plasma motion in the perpendicular direction too. In both configurations, the net effect of the magnetic field is to confine the plasma, opposing the expansion as a piston.

The subsonic evolution of a magnetized plasma in contact with a cold unmagnetized liner has been thoroughly analyzed in Chapters 3, 4 and 5. The motion presents a diffusive self-similar wave structure in the variable $x / \sqrt{t}$. When the magnetic pressure is negligible, the motion is governed by heat conduction. Liner material ablates and compresses the hot plasma, while the magnetic field is convected by the Nernst term towards the liner, where it diffuses in a thin layer. The Nernst term acts therefore as an effective engine that enhances the magnetic flux losses even when the plasma is highly conductive. The most efficient way to reduce its effect is to increase plasma magnetization, since it gets confined within in the cold unmagnetized plasma layer close to the liner. The energy and magnetic flux losses

are reduced with the plasma magnetization level $x_{e 0}$ as $x_{e 0}^{-7 / 10}$, whereas the liner ablated mass scales as $x_{e 0}^{-3 / 10}$.

When the magnetic pressure is important, the bulk of the diffusive wave is governed by Joule dissipation, while heat conduction is predominant close to the liner. The structure of the solution becomes insensitive to the magnetization level and is only governed by the magnetic Lewis number $\mathrm{Le}_{\mathrm{m}}$. A critical value of the latter, $\mathrm{Le}_{\mathrm{m}}=10$, 
has been identified below which plasma overheating due to Joule dissipation becomes important. The magnetic flux losses are no longer reduced as efficiently with $x_{e 0}$, and the liner mass ablation rate is notoriously enhanced by magnetic pressure gradients.

The structure of the diffusive wave changes appreciably when the liner material is different to the one of the hot plasma (fuel). If this is the case, a contact discontinuity develops at the interface between the ablated liner and the fuel, named ablated border. Mass density attains a higher value at the liner side of it, which may be susceptible to become Rayleigh-Taylor unstable. Additionally, the magnetic field is diffused in a thin layer at the fuel side, where its value decreases to adapt to the change the Nernst convection velocity. An asymptotic analysis in the large liner atomic number $Z_{2}$ limit reveals that mass ablation as well as thermal energy and magnetic flux losses decrease as $1 / \sqrt{Z_{2}}$. Liner-fuel mass diffusion has been studied next in the unmagnetized limit. It is a weaker transport process relative to heat conduction. Consequently, the mixing layer is thin compared to the heat wave and develops around the ablated border. The mass transport problem can therefore be computed a posteriori yielding accurate results. It is found that species concentration and ion pressure gradients are the predominant mechanisms leading to liner-fuel mass diffusion. The amount of liner material that pollutes the fuel can be reduced by increasing $Z_{2}$, and scales as $1 / \sqrt{Z_{2}}$. It accounts for a small percentage (between 2 and $5 \%$ ) of the total ablated liner mass, yet it can be comparable to the mixing induced by the turbulent layer arising from the Rayleigh-Taylor instability that can develop at the ablated border.

In Chapter 6, a detailed hot spot model of subsonic magnetized plasma implosions, relevant to free fall, deceleration and stagnation phases, has been derived. The physics of the cooling wave studied in the previous chapters takes place together with the implosion dynamics. Depending on the initial parameters, the implosion can follow a path towards a highly magnetized state, or fall into a superdiffusive regime where the hot spot gets demagnetized. In the first case, the core of the hot spot becomes magnetically insulated and undergoes an ideal adiabatic implosion. The ablated liner material penetrates until it encounters the highly magnetized region, where it accumulates. The temperature profile presents then a double ablation front like structure. The Nernst term is confined within the outer part of the hot spot. It sweeps away the magnetic field in this region, but leaves the magnetic field in the core of the hot spot intact, which is conserved throughout the implosion. This shows the importance to be placed in the magnetized regime parameter area rather than in the superdiffusive regime when designing magnetized implosions. The border between both regimes has been analytically derived for a small initial magnetization, yielding $\mathrm{Le}_{\mathrm{m} 0} \mathrm{Pe}_{0}^{10 / 7}=75$, with $\mathrm{Pe}_{0}$ standing for the initial Péclet number. This border steps back to lower values of $\mathrm{Le}_{\mathrm{m} 0}, \mathrm{Pe}_{0}$ when the initial magnetization is increased.

Finally, the dynamics of magnetized cylindrical implosions after stagnation has been investigated in Chapter 7. Alpha particles energy deposition plays a major role in this process. The expansion dynamics consists of a trade-off between alpha heating and expansion losses ( $\mathrm{PdV}$ work). When the target is unmagnetized, the expansion 
losses always dominate after a certain time, and the target undergoes an adiabatic expansion whose adiabat is determined by the conditions at stagnation. However, the presence of a magnetic field can modify the topology of the expansion. It confines the alpha particles inside the fuel and prevents them from escaping, enhancing the amount of energy deposited. For a sufficiently high magnetic field value at stagnation, alpha heating can dominate over the expansion losses, and the hot spot pressure presents an explosive behavior (singularity in a finite time). Neutron yield diverges in this case. Therefore, the conditions at stagnation for which this solution takes place can be taken as useful dynamic ignition criterion to measure the quality of the implosions. This criterion can be formulated in terms of the characteristic Lawson parameter and the magnetization of the alphas solely.

\subsection{Future work}

Several open questions arise related to every fundamental problem treated in this thesis. Regarding the magnetized plasma expansion, it would be interesting to study the stability of the discontinuity formed at the edge of the expansion when the magnetic field is perpendicular. Additionally, a detailed analysis of this structure for finite magnetic Reynolds number would improve the overall understanding of this phenomenon. For what concerns the evolution of a hot spot - liner interface, a major task would be the derivation of the transport coefficients for multi-component plasmas for an arbitrary magnetization value, and use this knowledge to study liner fuel mass diffusion in a more relevant context to magnetized liner inertial fusion. The hot spot model of magnetized plasma implosions can be substantially improved by including the magnetic pressure terms, which may become important by the end of the implosion. This can be done relatively straightforward using the simplification discussed in Chapter 4, which allows to derive an explicit expression for the ion velocity. Completing the model by letting the liner material be arbitrary and computing the amount of liner mass diffused into the fuel during an implosion would be another important improvement. Another key issue not studied in the literature corresponds to the stability of a magnetized cylindrical implosion. A simple model of a highly conductive plasma imploded by a non-conductive liner can serve as a first step analysis. Another potential source of instability would be the strong azimuthal electron current taking place at the outer part of the hot spot that supports the steep magnetic field gradient.This configuration can give rise to the development of a Weibel instability [90, 66]. Finally, several hypothesis assumed in the analysis of the dynamics after stagnation made in Chapter 7 would need further review. The most critical one is the assumption that the quotient between the radius of the hot spot and the fuel remains constant throughout the implosion. Although this may be accurate for highly magnetized implosions, it falls when the magnetization is not high enough. Consequently, a next step would correspond to the derivation of a more detailed model taking into account the dynamics of the ablated mass and 
consequently the heat conduction losses at the ablated liner - fuel interface. Subsequent improvements could be studying the effect of the Bremsstrahlung losses on the ignition criterion. 


\section{Bibliography}

[1] A. I. Akhiezer, I. A. Akhiezer, R. V. Polovin, and D. ter Haar. Plasma Electrodynamics: Linear Theory. Pergamon Press, 1975.

[2] H. Alfvén and E. Smårs. Nature, 188(4753):801-802, 1960.

[3] P. Amendt, O. L. Landen, H. F. Robey, C. K. Li, and R. D. Petrasso. Physical Review Letters, 105(11):115005, 2010.

[4] P. Amendt, S. C. Wilks, C. Bellei, C. K. Li, and R. D. Petrasso. Physics of Plasmas, 18(5):056308, 2011.

[5] D. Anderson, M. Bonnedal, and M. Lisak. Physica Scripta, 22(5):507, 1980.

[6] S. Atzeni and J. Meyer-Ter-Vehn. The Physics of Inertial Fusion: Beam Plasma Interaction, Hydrodynamics, Hot Dense Matter, volume 125. Oxford University Press on Demand, 2004.

[7] G. I. Barenblatt. Scaling, self-similarity, and intermediate asymptotics: dimensional analysis and intermediate asymptotics, volume 14. Cambridge University Press, 1996.

[8] D. H. Barnak, J. R. Davies, R. Betti, M. J. Bonino, E. M. Campbell, V. Yu. Glebov, D. R. Harding, J. P. Knauer, S. P. Regan, A. B. Sefkow, et al. Physics of Plasmas, 24(5):056310, 2017.

[9] M. M. Basko, A. J. Kemp, and J. Meyer-ter Vehn. Nuclear Fusion, 40(1):59, 2000.

[10] C. Bellei and P. A. Amendt. Physics of Plasmas, 24(4):040703, 2017.

[11] C. Bellei, H. Rinderknecht, A. Zylstra, M. Rosenberg, H. Sio, C. K. Li, R. Petrasso, S. C. Wilks, and P. A. Amendt. Physics of Plasmas, 21(5):056310, 2014 .

[12] D. Bennaceur-Doumaz and M. Djebli. Physics of Plasmas, 18(8):084507, 2011.

[13] R. Betti, K. Anderson, V. N. Goncharov, R. L. McCrory, D. D. Meyerhofer, S. Skupsky, and R. P. J. Town. Physics of Plasmas, 9(5):2277-2286, 2002.

[14] R. Betti, P. Y. Chang, B. K. Spears, K. S. Anderson, J. Edwards, M. Fatenejad, J. D. Lindl, R. L. McCrory, R. Nora, and D. Shvarts. Physics of Plasmas, 17(5):058102, 2010.

[15] R. Betti, A. R. Christopherson, B. K. Spears, R. Nora, A. Bose, J. Howard, K. M. Woo, M. J. Edwards, and J. Sanz. Physical Review Letters, $114(25): 255003,2015$. 
[16] R. Betti, V. N. Goncharov, R. L. McCrory, and C. P. Verdon. Physics of Plasmas, 2(10):3844-3851, 1995.

[17] R. Betti and O. A. Hurricane. Nature Physics, 12(5):435, 2016.

[18] R. Betti, M. Umansky, V. Lobatchev, V. N. Goncharov, and R. L. McCrory. Physics of Plasmas, 8(12):5257-5267, 2001.

[19] S. E. Bodner. Physical Review Letters, 33(13):761, 1974.

[20] S. I. Braginskii. Review of plasma physics, vol. 1. Consultants Bureau, New York, page 205, 1965.

[21] D. T. Casey, J. A. Frenje, M. Gatu Johnson, M. J. E. Manuel, H. G. Rinderknecht, N. Sinenian, F. H. Séguin, C. K. Li, R. D. Petrasso, P. B. Radha, J. A. Delettrez, V. Yu Glebov, D. D. Meyerhofer, T. C. Sangster, D. P. McNabb, P. A. Amendt, R. N. Boyd, J. R. Rygg, H. W. Herrmann, Y. H. Kim, and A. D. Bacher. Physical Review Letters, 108(7):075002, 2012.

[22] P. Y. Chang, G. Fiksel, M. Hohenberger, J. P. Knauer, R. Betti, F. J. Marshall, D. D. Meyerhofer, F. H. Séguin, and R. D. Petrasso. Physical Review Letters, 107(3):035006, 2011.

[23] A. R. Christopherson, R. Betti, A. Bose, J. Howard, K. M. Woo, E. M. Campbell, J. Sanz, and B. K. Spears. Physics of Plasmas, 25(1):012703, 2018.

[24] M. S. Chu. Physics of Fluids, 16(9):1441-1445, 1973.

[25] R. S. Craxton, K. S. Anderson, T. R. Boehly, V. N. Goncharov, D. R. Harding, J. P. Knauer, R. L. McCrory, P. W. McKenty, D. D. Meyerhofer, J. F. Myatt, et al. Physics of Plasmas, 22(11):110501, 2015.

[26] J. R. Davies, D. H. Barnak, R. Betti, E. M. Campbell, P.-Y. Chang, A. B. Sefkow, K. J. Peterson, D. B. Sinars, and M. R. Weis. Physics of Plasmas, 24(6):062701, 2017.

[27] F. S. Felber, M. A. Liberman, and A. L. Velikovich. Physics of Fluids, 31(12):3675-3682, 1988.

[28] F. S. Felber, M. A. Liberman, and A. L Velikovich. Physics of Fluids, 31(12):3683-3689, 1988.

[29] G. S. Fraley, E. J. Linnebur, R. J. Mason, and R. L. Morse. The Physics of Fluids, 17(2):474-489, 1974.

[30] S. Fujioka, Z. Zhang, K. Ishihara, K. Shigemori, Y. Hironaka, T. Johzaki, A. Sunahara, N. Yamamoto, H. Nakashima, T. Watanabe, H. Shiraga, H. Nishimura, and H. Azechi. Scientific Reports, 3:1170, 2013.

[31] S. F. Garanin. Journal of Applied Mechanics and Technical Physics, 28(6):816819, 1987.

[32] S. F. Garanin and V. I. Mamyshev. Journal of Applied Mechanics and Technical Physics, 31(1):28-34, 1990. 
[33] J. Garnier and C. Cherfils. A multiscale analysis of the hotspot dynamics during the deceleration phase of inertial confinement capsules. Physics of Plasmas, 12(1):012704, 2005.

[34] M. R. Gomez, S. A. Slutz, A. B. Sefkow, D. B. Sinars, K. D. Hahn, S. B. Hansen, E. C. Harding, P. F. Knapp, P. F. Schmit, C. A. Jennings, et al. Physical Review Letters, 113(15):155003, 2014.

[35] V. N. Goncharov. Physical Review Letters, 82(10):2091, 1999.

[36] O. V. Gotchev, P. Y. Chang, J. P. Knauer, D. D. Meyerhofer, O. Polomarov, J. Frenje, C. K. Li, M. J. E. Manuel, R. D. Petrasso, J. R. Rygg, et al. Physical Review Letters, 103(21):215004, 2009.

[37] O. V. Gotchev, N. W. Jang, J. P. Knauer, M. D. Barbero, R. Betti, C. K. Li, and R. D. Petrasso. Journal of Fusion Energy, 27(1-2):25-31, 2008.

[38] O. V. Gotchev, J. P. Knauer, P. Y. Chang, N. W. Jang, M. J. Shoup III, D. D. Meyerhofer, and R. Betti. Review of Scientific Instruments, 80(4):043504, 2009.

[39] A. V. Gurevich, L. V. Pariiskaya, and L. P. Pitaevskii. Soviet Phyics. JETP, 22(2):449-454, 1966.

[40] F. Hamann, P. Combis, and L. Videau. Physics of Plasmas, 22(8):082708, 2015.

[41] E. Haug and W. Nakel. The Elementary Process of Bremsstrahlung, volume 73. World Scientific, 2004.

[42] R. D. Hazeltine and F. L. Waelbroeck. The Framework of Plasma Physics. Reading, MA, 1998.

[43] P. Helander and D. Sigmar. Collisional transport in magnetized plasmas, volume 4. Cambridge University Press, 2005.

[44] M. Hohenberger, P. Y. Chang, G. Fiksel, J. P. Knauer, R. Betti, F. J. Marshall, D. D. Meyerhofer, F. H. Séguin, and R. D. Petrasso. Physics of Plasmas, 19(5):056306, 2012.

[45] S. F. Johnson and K. E. Lonngren. Physica Scripta, 25(4):583, 1982.

[46] R. D. Jones and W. C. Mead. Nuclear fusion, 26(2):127, 1986.

[47] G. Kagan and X. Z. Tang. Physics of Plasmas, 19(8):082709, 2012.

[48] G. Kagan and X. Z. Tang. Physics of Plasmas, 21(2):022708, 2014.

[49] G. Kagan and X. Z. Tang. Physics Letters A, 378(21):1531, 2014.

[50] R. C. Kirkpatrick, I. R. Lindemuth, and Marjorie S. Ward. Fusion Science and Technology, 27(3):201-214, 1995.

[51] J. P. Knauer, O. V. Gotchev, P. Y. Chang, D. D. Meyerhofer, O. Polomarov, R. Betti, J. A. Frenje, C. K. Li, M. J.-E. Manuel, R. D. Petrasso, et al. Physics of Plasmas, 17(5):056318, 2010. 
[52] O. N. Krokhin and V. B. Rozanov. Soviet Journal of Quantum Electronics, 2(4):393, 1973.

[53] H. J. Kull. Physics of Fluids B: Plasma Physics, 1(1):170-182, 1989.

[54] L. D. Landau and E. M. Lifshitz. Fluid Mechanics. Pergamon Press, 1959.

[55] L. D. Landau and E. M. Lifshitz. Electrodynamics of Continuous Media. Pergamon Press, 1960.

[56] R. Landshoff. Physical Review, 76(7):904, 1949.

[57] J. D. Lawson. Proceedings of the Physical Society. Section B, 70(1):6, 1957.

[58] I. R. Lindemuth and R. C. Kirkpatrick. Nuclear Fusion, 23(3):263, 1983.

[59] J. D. Lindl. Inertial confinement fusion: the quest for ignition and energy gain using indirect drive. American Institute of Physics, 1998.

[60] J. D. Lindl, P. Amendt, R. L. Berger, S. G. Glendinning, S. H. Glenzer, S. W. Haan, R. L. Kauffman, O. L. Landen, and L. J. Suter. Physics of Plasmas, 11(2):339-491, 2004.

[61] E. E. Meshkov. Fluid Dynamics, 4(5):101-104, 1969.

[62] K. Miyamoto. Plasma Physics for Nuclear Fusion Research. MIT Press, Cambridge, 1988.

[63] K. Molvig, A. N. Simakov, and E. L. Vold. Physics of Plasmas, 21(9):092709, 2014.

[64] K. Molvig, E. L. Vold, E. S. Dodd, and S. C. Wilks. Physical Review Letters, 113(14):145001, 2014.

[65] P. Mora. Physical Review Letters, 90(18):185002, 2003.

[66] R. L. Morse and C. W. Nielson. The Physics of Fluids, 14(4):830-840, 1971.

[67] J. Nuckolls, L. Wood, A. Thiessen, and G. Zimmerman. Nature, 239(5368):139, 1972.

[68] R. D. Richtmyer. Communications on Pure and Applied Mathematics, 13(2):297-319, 1960.

[69] D. C. Rovang, D. C. Lamppa, M. E. Cuneo, A. C. Owen, J. Mckenney, D. W. Johnson, S. Radovich, R. J. Kaye, R. D. McBride, C. S. Alexander, et al. Review of Scientific Instruments, 85(12):124701, 2014.

[70] L. I. Rudakov, A. Chuvatin, A. L. Velikovich, and J. Davis. Physics of Plasmas, 10(11):4435-4447, 2003.

[71] J. J. Santos, M. Bailly-Grandvaux, L. Giuffrida, P. Forestier-Colleoni, S. Fujioka, Z. Zhang, P. Korneev, R. Bouillaud, S. Dorard, D. Batani, et al. New Journal of Physics, 96(2):083051, 2015.

[72] J. Sanz. Physical Review Letters, 73(20):2700, 1994. 
[73] J. Sanz and R. Betti. Physics of Plasmas, 12(4):042704, 2005.

[74] J. Sanz, J. Garnier, C. Cherfils, B. Canaud, L. Masse, and M. Temporal. Physics of Plasmas, 12(11):112702, 2005.

[75] J. Sanz, A. R. Piriz, and F. G. Tomasel. Self-similar model for tamped ablation driven by thermal radiation. Physics of Fluids B: Plasma Physics, 4(3):683-692, 1992.

[76] J. Sanz, J. Ramirez, R. Ramis, R. Betti, and R. P. J. Town. Physical Review Letters, 89(19):195002, 2002.

[77] A. J. Schmitt. Applied Physics Letters, 44(4):399-401, 1984.

[78] A. J. Schmitt and J. H. Gardner. Journal of Applied Physics, 60(1):6-13, 1986.

[79] A. B. Sefkow, S. A. Slutz, J. M. Koning, M. M. Marinak, K. J. Peterson, D. B. Sinars, and R. A. Vesey. Physics of Plasmas, 21(7):072711, 2014.

[80] A. N. Simakov and K. Molvig. Physics of Plasmas, 21(2):024503, 2014.

[81] A. N. Simakov and K. Molvig. Physics of Plasmas, 23(3):032115, 2016.

[82] A. N. Simakov and K. Molvig. Physics of Plasmas, 23(3):032116, 2016.

[83] S. Skupsky and K. Lee. Journal of Applied Physics, 54(7):3662-3671, 1983.

[84] S. A. Slutz, M. C. Herrmann, R. A. Vesey, A. B. Sefkow, D. B. Sinars, D. C. Rovang, K. J. Peterson, and M. E. Cuneo. Physics of Plasmas, 17(5):056303, 2010 .

[85] S. A. Slutz and R. A. Vesey. Physical Review Letters, 108(2):025003, 2012.

[86] H. Takabe, K. Mima, L. Montierth, and R. L. Morse. The Physics of Fluids, 28(12):3676-3682, 1985.

[87] E. Teller. Fusion. Volume 1. Magnetic confinement. Academic Press, Inc., New York, NY, 1981.

[88] V. T. Tikhonchuk, M. Bailly-Grandvaux, J. J. Santos, and A. Poyé. Physical Review E, 96(2):023202, 2017.

[89] A. L. Velikovich, J. L. Giuliani, and S. T. Zalesak. Physics of Plasmas, 22(4):042702, 2015.

[90] E. S. Weibel. Physiical Review Letters, 2:83-84, Feb 1959.

[91] M. M. Widner, J. Chang, A. V. Farnsworth, R. J. Leeper, T. S. Prevender, L. Baker, and J. N. Olsen. In Bulletin of the American Physical Society, volume 22, pages 1139-1139, 1977.

[92] G. A. Wurden, S. C. Hsu, T. P. Intrator, T.C. Grabowski, J. H. Degnan, M. Domonkos, P. J. Turchi, E. M. Campbell, D. B. Sinars, M. C. Herrmann, et al. Journal of Fusion Energy, 35(1):69-77, 2016. 
[93] Y. B. Zel'dovich and Y. P. Raizer. Physics of shock waves and high-temperature hydrodynamic phenomena. Dover Publ., 2002.

[94] V. M. Zhdanov. Transport processes in multicomponent plasma. CRC Press, 2014.

[95] C. D. Zhou and R. Betti. Physics of Plasmas, 14(7):072703, 2007.

[96] C. D. Zhou and R. Betti. Physics of Plasmas, 15(10):102707, 2008. 